$1 \%$

fin.

$\int^{2}: \frac{1}{2}-y^{2}$

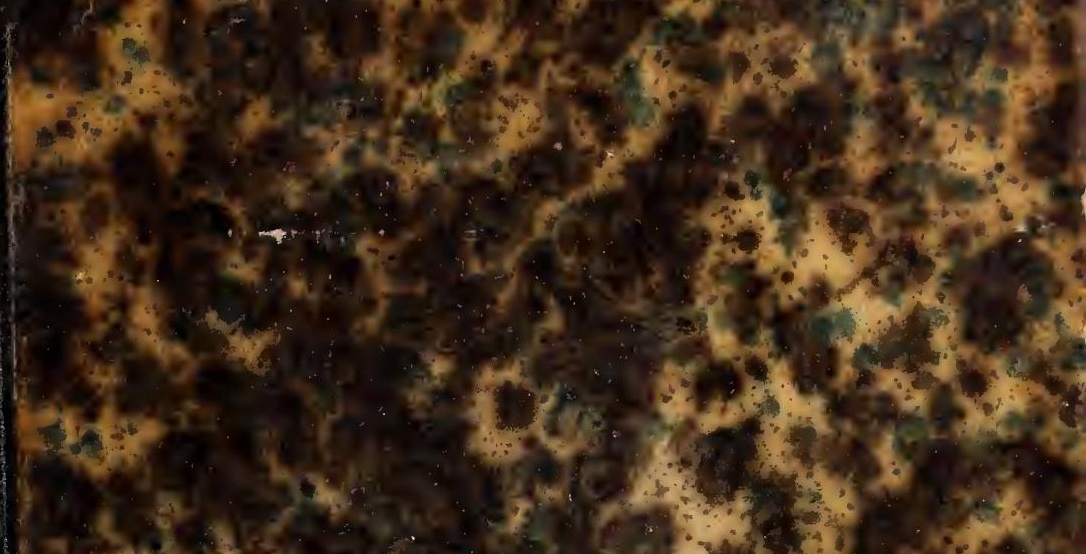

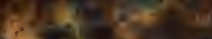

Sic

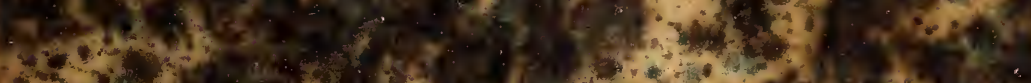

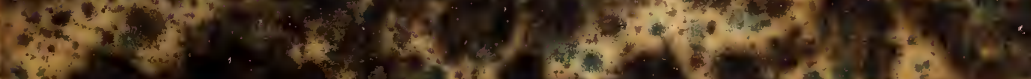
(1)

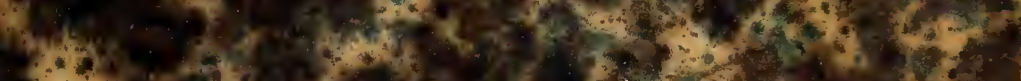

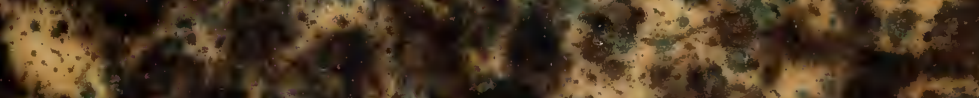

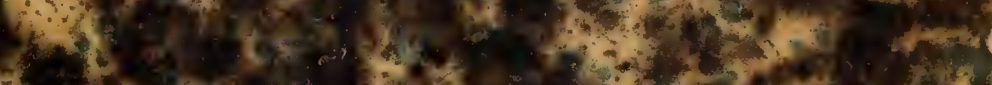

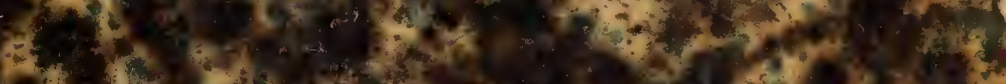

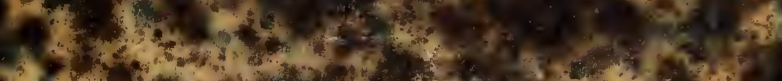

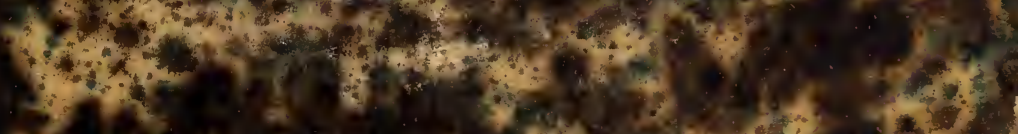

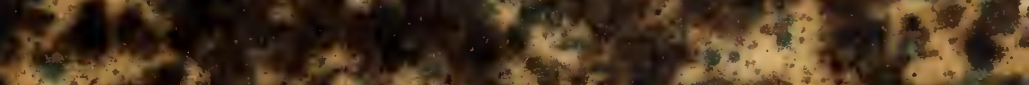

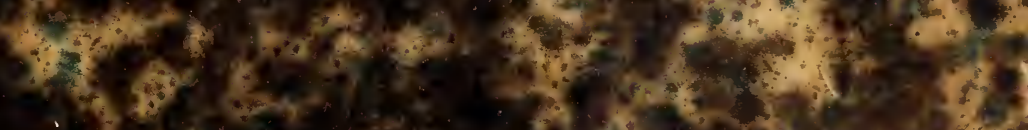
Xation

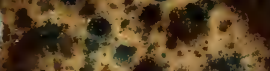

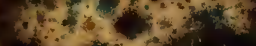
asent?
- (1)

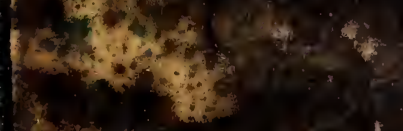

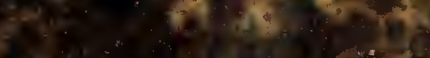
extexwas:
(a)

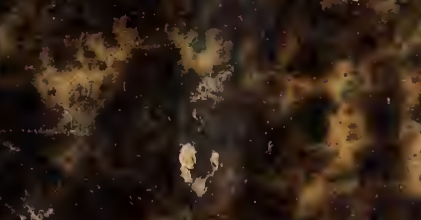

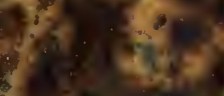
$\therefore \rightarrow$
(a)

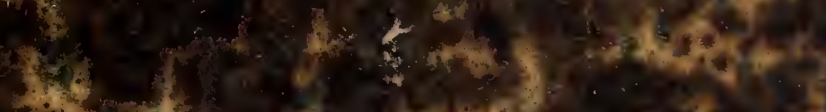

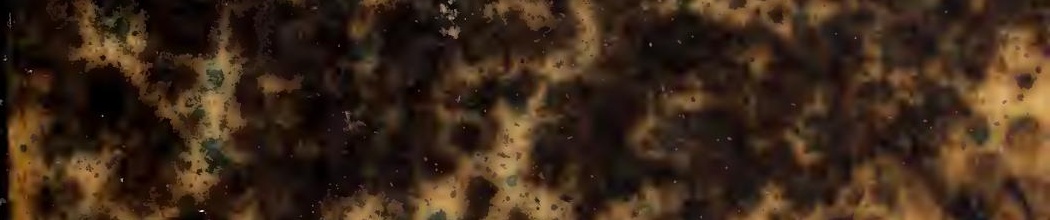




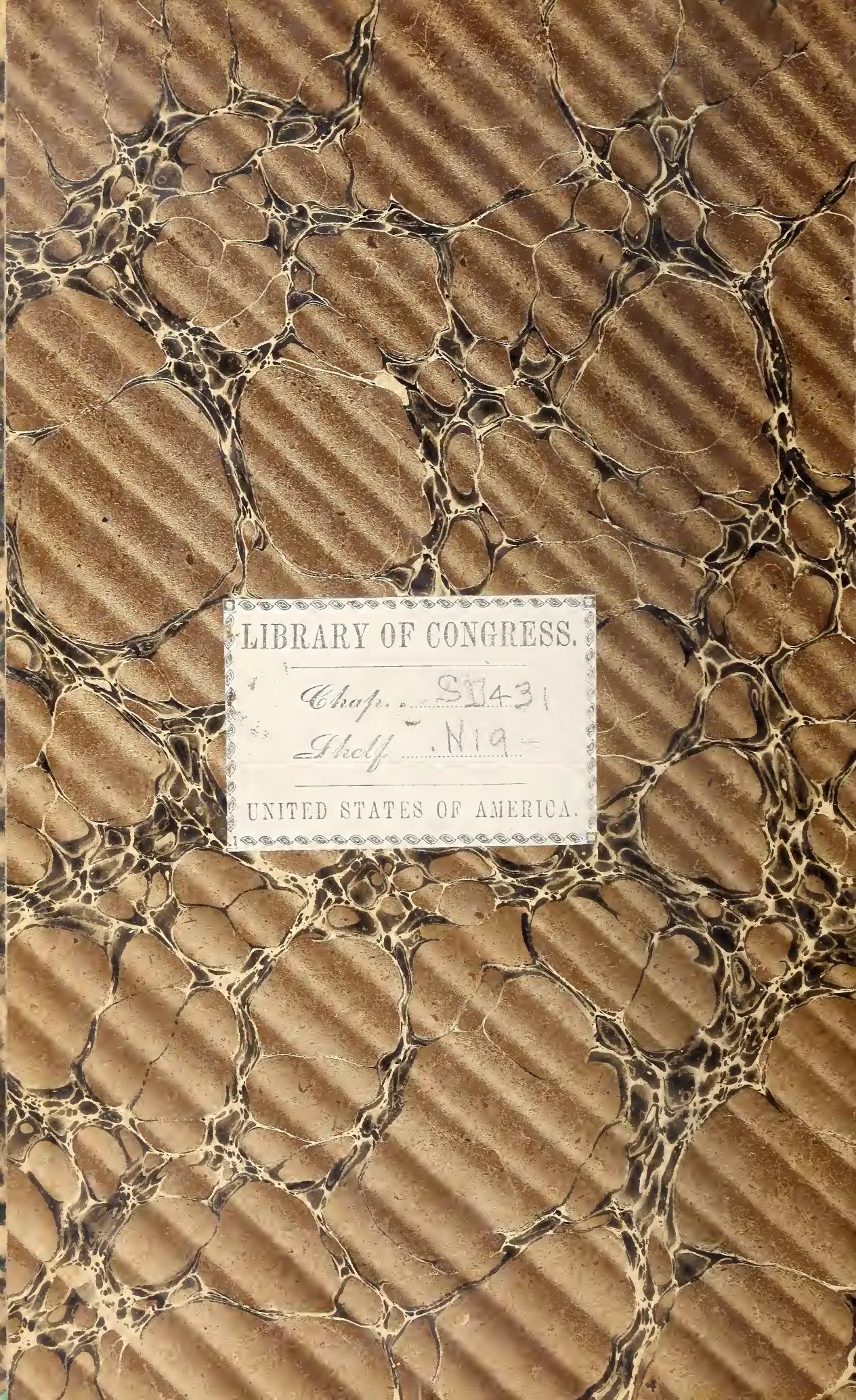




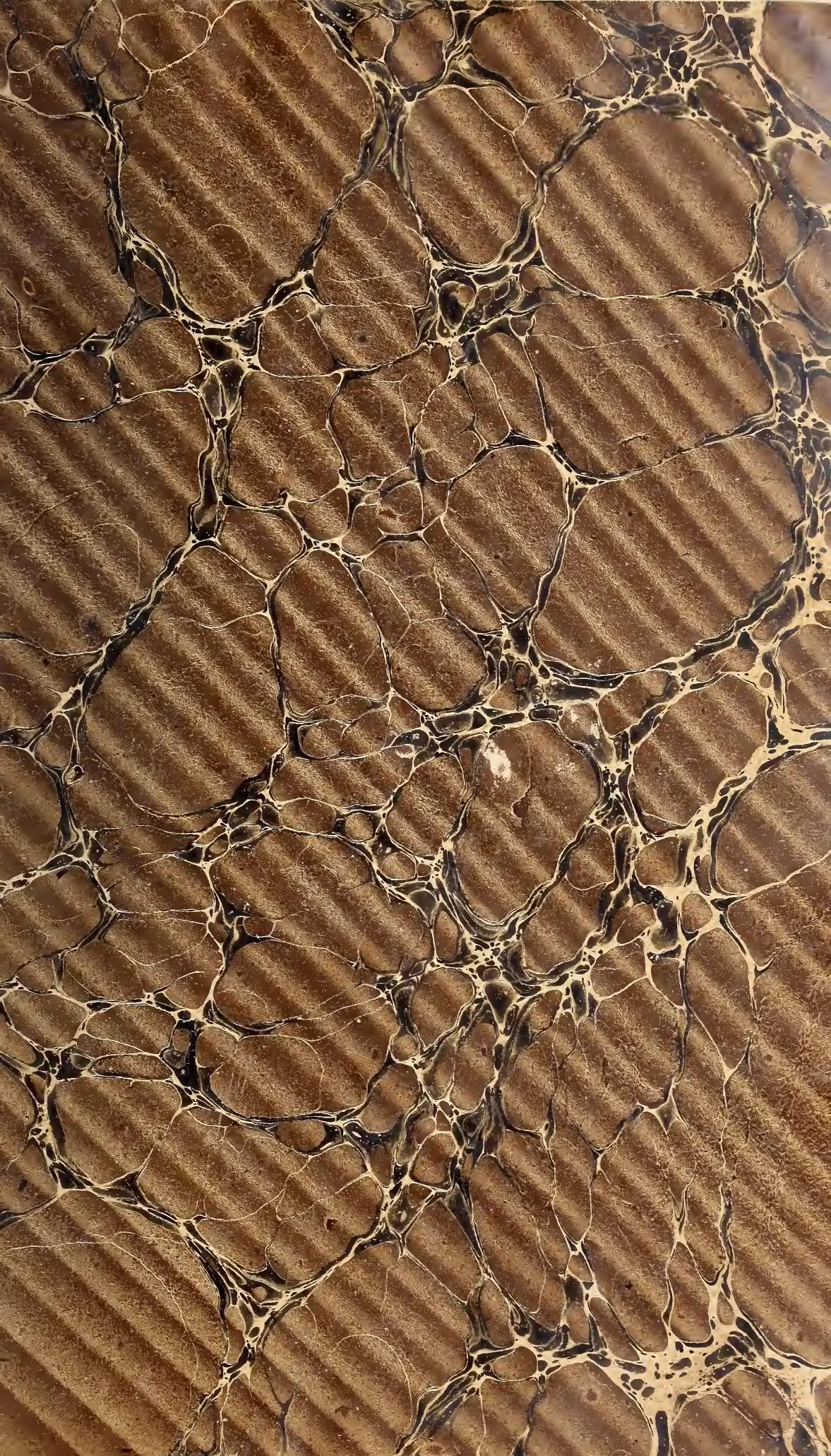




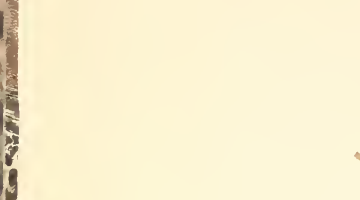

.

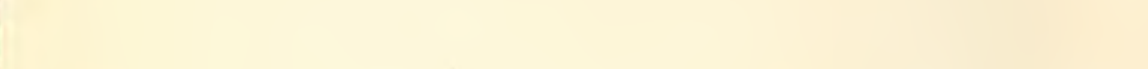





\section{EXPLOITATION}

DÉBIT ET ESTIMATION

\section{DES BOIS}


Nancy, imprimerie de ve Raybois, rue du faub. Stanislas, 5 . 


\section{EXPLOITATION}

\section{DÉBIT ET ESTIMATION DES BOIS}

Cours créé à l'École impériale forestière

PAR

\section{H. NANQUETTE}

Conservateur des forêts, Directeur de l'Ecole impériale forestière Ancien élève de cette Ecole.

$2^{\circ}$ ÉDITION.

NANGY

Ve RAYBOIS, INPRIMEUR DE L'ÉCOLE FORESTIĖRE Rue du faubourg Stanislas, 3 


\section{INTRODUCTION}

Les produits forestiers se divisent, comme on le sait, en deux grandes catégories : les bois de chauffage et les bois d'œuvre. C'est du débit de ces bois et de l'emploi qu'on en peut faire, selon les dimensions et la qualité, qu'il sera particulièrement question dans la première partie de cet ouvrage. La seconde partie contiendra la description des procédés d'estimation des bois sur pied et l'exposé des principes suivant lesquels on détermine la valeur des forèts en fonds et superficie.

Ainsi défini, ce livre n'est point, ne peut pas être, une œuvre de science. Toutefois il touche par beaucoup de points à une question économique de la plus haute importance, celle de l'approvisionnement de la France en produits 
forestiers. Cette question, je ne puis la développer ici, parce que les matériaux me manquent pour la discuter; mais je tiens au moins à l'indiquer et à faire connaitre les préoccupations qu'elle entretient dans mon esprit.

Sous la dénomination de bois de chaufage, ou de bois de feu, on comprend les produits qui servent à l'alimentation des foyers industriels et domestiques. Tels sont les bois de quartier et de rondin, les fagots et le bois à charbon. Jusqu'à présent, nos forêts, tant celles qui sont soumises au régime forestier que celles appartenant aux particuliers, ont pu suffire à la consommation du pays. La valeur vénale des bois de feu n'a même pas beaucoup changé depuis un quart de siècle, malgré l'enchérissement qui s'est produit sur presque tous les objets de consommation. Cela tient sans doute à ce que le combustible minéral s'est substitué au bois dans un grand nombre d'emplois; mais cela tient aussi à ce que toutes les forêts produisent du bois de feu, qu'un grand nombre d'entre elles ne sont même cultivées qu'en vue de ce genre de produits, et que tous les bois d'œuvre, en général, aboutissent avec le temps à la même fin. Ni les forestiers, ni les économistes ne se sont donc préoccupés de la 
production des bois de feu en France, et il ne parait pas que, de longtemps du moins, ce genre de combustible fasse défaut à la consommation.

En est-il de même des bois d'œuvre? - Ces bois sont ceux que l'on désigne communément sous le nom de bois de service quand ils doivent être employés comme charpentes dans les constructions civiles ou navales, et que l'on appelle bois de travail ou d'industrie quand ils sont débités en planches, en merrains, etc., pour les besoins de la menuiserie, de l'ébénisterie, de la tonnellerie, etc. En France, ce sont les forèts de chène et de sapin qui produisent et produiront toujours les bois les plus propres aux constructions, comme aussi ce sont les forêts de chêne et de sapin qui fourniront toujours les meilleurs bois de menuiserie, de tonnellerie, etc. - Or, tandis que les sapinières sont forcément reléguées dans les climats froids ou rudes des hautes montagnes, parce que c'est dans ces climats seulement que le bois de sapin acquiert de la qualité, le chêne, au contraire, ne peut être cultivé avec avantage que dans les plaines et sur les coteaux de moyenne élévation. Nos deux principales essences doivent donc être parquées, pour ainsi dire, dans des régions climatériques très-différentes. En outre, tandis que le 
sapin se plaît surtout dans les terrains légers, rocheux, impropres à l'agriculture, le chêne ne prospère que dans les sols frais ou humides, profonds, qui renferment une assez forte proportion d'argile en mélange avec du sable siliceux ou calcaire. Ces derniers terrains sont le plus souvent ceux des plaines et des coteaux propres à la culture des céréales, des prairies, de la vigne et des plantes industrielles, et c'est ce qui explique pourquoi les forêts qui les couvraient ont disparu progressivement avec la marche de la civilisation, tandis que les sapinières ont été mieux conservées et couvrent encore une grande partie des montagnes qu'elles occupaient originairement. Cependant, là aussi, des étendues assez considérables de forêts résineuses ont fait place à des pâturages, au grand avantage des populations qui ont su en limiter la jouissance, mais le plus souvent au détriment de celles qui en ont abusé.

Etant bien établi que le chêne est un arbre des terrains frais et fertiles des plaines et des coteaux, que le sapin est l'essence des climats froids et des sols légers des contrées montagneuses, que ces deux espèces peuvent, à elles seules, pourvoir aux besoins les plus indispensables de la consommation; sachant d'ailleurs que, pour produire des 
pièces de fortes dimensions, la culture de ces deux grandes essences exige de longues révolutions et ne peut ètre entreprise que par des propriétaires impérissables, on se demande : $1^{\circ}$ si les forêts domaniales et communales appartenant à chacun de ces deux groupes sont bien réparties sur le sol français, et si ellès y occupent une place suffisante; $2^{\circ}$ si les produits qu'elles fournissent aujourd'hui, et qu'elles pourront fournir dans l'avenir, suffisent maintenant ou suffiront toujours aux besoins du pays.

La réponse à ces deux questions n'est que trop facile à faire; elle n'est pas rassurante pour l'avenir.

Un coup d'œil jeté sur la carte forestière de France nous montre, en effet, que les forèts sont très-inégalement distribuées sur le territoire de l'Empire, et que, à l'exception de la Flandre, contrée fertile par excellence et située dans un climat exceptionnellement humide, les provinces les plus riches, les plus prospères, les plus industrieuses et les plus peuplées de notre pays sont celles qui ont conservé le plus de forêts. Un coup d'œil jeté sur la carte du monde ancien nous montre de même que si le défrichement progressif des forêts a marché de front avec la 
civilisation, la suppression absolue de la culture forestière a marqué, presque partout, l'époque de la décadence des peuples. Pour ne citer qu'un exemple, qui ne sait que le berceau du christianisme, cette Terre Promise où prospéraient de nombreuses populations, est devenu un aride désert après la disparition des forêts qui en faisaient la richesse et l'ornement?

Quant au chiffre de la production forestière française et à l'étendue de nos besoins en bois de fortes dimensions, personne ne les connait; ces renseignements n'existent nulle part, et j'éproure un véritable regret de n'aroir pu les introduire dans la seconde édition de ce modeste ourrage. Mais on sait, d'une manière certaine, que la quantité de bois nécessaire à l'entretien de notre flotte et de nos voies ferrées n'absorbe pas moins de 400,000 mètres cubes par année, ce qui équivaut à la possibilité de plus de 100,000 hectares de futaie de chêne en plein rapport. D'autre part, les tableaux officiels de l'administration des douanes nous apprennent que l'excédant de l'importation sur l'exportation des bois communs de service et d'industrie représente, annuellement, une valeur d'environ 150 millions de francs, et que dans cette somme le merrain destiné à la 
fabrication des tonneaux entre pour 45 millions.

A ne considérer que ces derniers chiffres, on voit que nous demandons à l'étranger des bois d'œuvre pour une somme considérable. Que si, comme il faut le désirer, on parvient à substituer le fer au bois, sans trop d'inconvénient, dans la plus grande partie des constructions, il n'en reste pas moins acquis que nous ne pouvons plus faire face à tous les besoins de la menuiserie, même en bois communs comme ceux de sapin et d'autres espèces résineuses; qu'enfin, l'une des branches les plus importantes de la production nationale, l'industrie viticole, ne trouve plus sur le sol français le merrain de chène nécessaire pour loger et exporter ses produits.

Sans doute nous pourrions, comme certairs économistes, ne pas nous inquiéter de cet état de choses, si les bois étrangers, dont la quantité importée augmente chaque année, pouvaient nous être fournis avec certitude dans les temps à venir. Mais cette assurance n'est rien moins que positive, et si nous interrogeons les États tels que la Suède, la Norwége et les autres contrées de la Baltique, d'où nous recevons la plus grande partie des bois résineux étrangers qui entrent dans notre consommation, ils nous répondent, par leurs voix les 
plus autorisées, que leurs forêts s'épuisent et que, du train dont on marche, elles ne suffiront bientôt plus à leurs propres besoins. La Russie elle-mème, avec ses immenses forêts, ne peut déjà plus nous offrir qu'à des prix élevés ces bois de pin, si précieux pour la charpente et la menuiserie, qu'elle nous livrait naguère en si grande abondance; car, il ne faut pas s'abuser, il n'y a que les forêts d'un accès facile, comme celles du littoral de la Baltique, qui puissent être exploitées avec profit pour l'exportation. Et d'ailleurs nous ne sommes pas les seuls qui puisions à cette source. Les Anglais nous y ont précédés et ils continueront à s'y alimenter aussi longtemps qu'ils trouveront ce marché approvisionné. Mais ils comprennent que les forêts du nord de l'Europe s'appauvrissent de jour en jour et que l'avenir n'est point assuré de ce côté. C'est pourquoi le gouvernement, justement préoccupé des besoins de la nation, s'est attaché, depuis quelque temps déjà, à organiser une administration qui a pour mission de régulariser les exploitations des bois résineux de l'Himalaya et de réparer les ruines causées par la conquête dans les forèts de plaine de l'Inde anglaise.- Quant aux bois de chêne, nous les tirons, sous la forme de merrains, des provinces danu- 
biennes de l'Autriche et de quelques forêts voisines du Rhin, ou bien, en pièces isolées de charpente, de l'Italie et de la Sardaigne, pour quelques besoins spéciaux de nos arsenaux maritimes. Mais qu'une guerre éclate entre la France et l'Allemagne, et c'est jusqu'en Roumanie et en Turquie que nos viticulteurs iront chercher les produits nécessaires à leur industrie. Car, s'il est possible de remplacer le bois par le fer dans les constructions, s'il est permis d'espérer que l'on parviendra bientôt à réduire considérablement l'emploi du bois dans l'établissement, l'entretien et l'exploitation des chemins de fer, personne n'a encore songé à remplacer le chêne par une autre substance dans la fabrication des tonneaux destinés à loger nos vins et nos eaux-de-vie.

Je conclus qu'il faut conserver les forèts qui nous restent, les forêts de plaine surtout; qu'il faut améliorer celles dont la production laisse à désirer, et en créer de nouvelles dans les provinces qui ont eu la fatale incurie de les laisser détruire. Depuis quelques années l'administration a fait de louables efforts pour arrêter la dévastation de nos hautes montagnes et conjurer la ruine des populations qui vivent agglomérées à leur pied ou disséminées sur leur flanc. L'œuvre est grande et 
suffit pour recommander à la postérité l'homme d'Etat qui l'a conçue. Mais qu'on ne l'oublie pas : il faut près de deux siècles, et souvent plus, pour conduire à leur exploitabilité les forêts que l'on crée dans les pays de montagnes. D'ailleurs, quand tous les pâturages des Alpes seraient rendus à la culture forestière, cela ne donnerait pas un chêne de plus à la consommation; car, j'insiste à dessein sur ce point, ce n'est que dans les plaines et sur les coteaux, dans des terrains frais, profonds et relativement fertiles que l'on peut cultiver le chêne avec succès.

NANQUETTE.

Nancy, avril 1869. 


\section{EXPLOITATION}

DÉBIT ET ESTIMATION

DES BOIS 



\title{
PREMIERE PARTIE
}

\section{EXPLOITATION}

\section{DEBIT ET CONSERVATION \\ DES BOIS}

\section{CHAPITRE PREMIER.}

\author{
ABATAGE DES BOIS.
}

Article I.

Abatage des bois dans les taillis.

L'abatage des bois, dans les taillis, doit se faire avec des instruments bien tranchants, afin de ne pas faire éclater la souche et l'écorce qui la recouvre. Les perches ayant un décimètre de tour et audessus doivent être coupées à la hache; pour les brins plus faibles, il est préférable d'employer la serpe, afin d'éviter l'ébranlement et souvent la rupture des racines que le choc de la hache occasionne aisément. On peut même se servir, pour les tiges les plus minces, de la scie, en ayant soin de faire la section oblique à l'horizon. Ces précautions sont 
recommandées, en vue d'assurer le mieux possible la reproduction des souches (1).

En général, on coupe le plus près possible de terre, et l'on donne à la section d'abatage une forme telle que les eaux pluviales ne puissent séjourner sur les souches. C'est ce que l'on nomme exploiter en talus. Dans l'opération de l'abatage, les ouvriers dirigent la chute des arbres de manière à les faire tomber les uns sur les autres, afin de ne pas embarrasser le bois qui n'est pas abattu.

La saison qui serait la plus convenable pour l'abatage des taillis est le moment qui précède immédiatement les premiers mouvements de la séve du printemps; on sait pourquoi. Mais la rareté des bûcherons et la nécessité d'assurer du travail à ces ouvriers pendant les plus mauvais temps de l'année s'opposent à l'application de ce précepte, et ont fait prévaloir l'ancienne habitude d'exploiter les taillis pendant le temps qui s'écoule entre le moment où la végétation s'arrête et celui où la séve commence à se remettre en mouvement. L'abatage a lieu, par conséquent, entre le mois d'octobre et le 15 avril suivant, époque à laquelle, d'après le cahier des charges, l'opération doit être entièrement terminée. Quant au moment où l'abatage peut commencer, il est déterminé, dans chaque localité et pour chaque coupe, par le permis d'exploiter que le chef de service délivre aux adjudicataires, sur la présentation des pièces exigées par le cahier des charges.

(1) MMI. Lorentz et Parade, Cubture des Bois. 
ARticle II.

Abatage des bois dans les futaies.

Dans les futaies, l'abatage des arbres n'exige pas les mêmes soins que dans les taillis, parce que la régénération devant avoir lieu par la semence, on n'a pas à se préoccuper de ménager les souches en vue de la production des rejets. Les arbres de futaie s'exploitent à la hache, ou avec une scie particulière que tout le monde connait et que l'on nomme passepartout. Dans toutes les circonstances où l'on peut faire usage de la scie, il est préférable de l'employer, afin d'éviter la perte qui résulte de l'entaille que l'on est obligé de faire, lorsqu'on se sert de la hache. Cette perte est d'autant plus forte que les arbres sont plus gros, parce que la hauteur de l'entaille est plus considérable. Aussi, dans les localités où l'usage de la scie n'a pas encore pénétré, voit-on les bûcherons, lorsqu'ils y sont autorisés, déraciner les arbres à une certaine profondeur et commencer l'entaille au dessous du collet des racines. Cette pratique ne doit évidemment être généralisée que dans les coupes où elle ne peut causer aucun dommage aux peuplements environnants, par exemple, dans certaines coupes d'ensemencement où il n'existe pas de semis.

Dans les coupes secondaires et définitives et dans les coupes d'extraction de vieux arbres en plein massif, on prend aussi la précaution de faire ébrancher les arbres jusqu'à la cime avant l'abatage, afin qu'ils 
causent le moins de dommage possible par leur chute. On fait de même dans les coupes de taillis sous futaie et dans les coupes d'ensemencement, pour éviter que les arbres, en tombant, n'endommagent les arbres réservés. Enfin, dans les terrains en pente, l'ouvrier cherche à diriger l'arbre du côté du sommet, pour empêcher qu'il ne se brise.

II existe des doutes sur la saison la plus favorable à l'abatage des bois de futaie. - L'opinion ancienne, qui prévaut encore le plus souvent et qui sert de règle aux exploitations, considère comme la meilleure la fin de l'automne et l'hiver. On dit que, coupés en saison morte, les bois ont une plus longue durée lorsqu'ils sont mis en cuvre ; il semble certain aussi qu'employés au chauffage ils brûlent plus facilement et donnent même plus de chaleur que ceux qui ont été abattus dans le temps de la végétation.

Cependant les bois résineux ne paraissent pas soufrrir dans leur qualité de la coupe en été. C'est dans cette saison qu'ils s'exploitent, de temps immémorial dans les Vosges, et cette règle tend de plus en plus à se généraliser dans les forêts peuplées d'arbres verts. Beaucoup de praticiens éclairés, d'accord avec les données de la physiologie, sont d'avis qu'en prenant la précaution de les écorcer aussitôt après l'abatage, les bois se dessèchent plus facilement et plus complétement, qu'ils sont moins sujets à se tourmenter, d'un plus bel aspect quant à la blancheur et à l'éclat, plus légers enfin et, par suite, d'm transport plus économique. 
Ce résultat d'expérience est de nature à ébranler l'opinion accréditée relativement aux bois feuillus et semblerait aussi militer en faveur de l'exploitation de ces bois en été, après le mouvement de la séve, sinon en ce qui concerne leur valeur calorifique, du moins au point de vue de leur durée. Cependant il serait imprudent de se prononcer dès à présent, et, jusqu'à ce que des expériences nombreuses, précises et comparables aient décidé la question définitivement, il semble convenable de suivre les errements anciens. Il est probable d'ailleurs que la saison d'abatage n'exerce d'action que sur les bois qui restent plus ou moins complétement à l'état d'aubier pendant toute leur vie, ou sur l'aubier des arbres qui présentent un bois parfait bien constitué.

Après leur abatage, les bois prennent un retrait plus ou moins rapide, selon leur qualité, la saison dans laquelle ils ont été coupés, et l'état de l'atmosphère dans les lieux où ils sont déposés. Cette contraction des fibres, due à l'évaporation d'une partie de la séve que renferme le bois, occasionne des fentes qui peuvent être assez fortes pour altérer la solidité de la pièce et la rendre impropre à certains usages. D'un autre côté, cette évaporation ajoute à la qualité des bois d'œuvre. Il importe donc qu'elle ait lieu lente ment pour éviter des fentes trop fortes, et aussitôt après l'abatage, pour préserver les bois, autant qu'on le peut, des effets de la fermentation. L' abatage des arbres en hiver remplit assez bien ces 
conditions, parce qu'alors la fermentation est moins active, la dessiccation s'opère petit à petit, et l'arbre peut arriver à l'été déjà assez desséché, pour ne plus craindre les chaleurs de cette saison. Mais pour les bois feuillus, quand l'abatage a lieu en été, on évite d'écorcer les arbres aussitôt qu'ils sont abattus, et on les place, autant que possible, en lieu sec et à l'ombre. Ces précautions sont surtout nécessaires dans les pays chauds; elles sont toujours faciles à prendre dans les coupes et, pour les grosses pièces difficiles à manier, on peut les mettre à l'abri des ardeurs du soleil en les couvrant de débris quelconques de l'exploitation.

\section{Article III.}

Abatage des bois dans les coupes d'amélioration.

On donne le nom de coupes d'amélioration aux coupes de nettoiement et d'éclaircie que l'on fait dans les taillis et dans les futaies, pour favoriser la croissance des arbres qui doivent rester sur pied jusqu'au moment des coupes principales. Nous comprendrons aussi dans cette catégorie les opérations d'élagage qui sont de véritables exploitations d'amélioration.

La coupe de nettoiement est une opération qui a pour objet de dégager les sujets d'essence précieuse des obstacles apportés à leur végétation par les morts bois et les bois tendres ou autres dont la croissance est plus active dans la jeunesse, et qui, par leur cou- 
vert, entravent ou menacent d'entraver le développement des premiers. Cette coupe consiste, par consćquent, dans la suppression ou la mutilation d'une partie des sujets qui composent un massif, au profit de ceux que l'on veut maintenir. C'est assez dire que dans ces exploitations il n'y a d'autre précaution à prendre, en ce qui concerne la coupe ou l'abatage des bois, que celle nécessaire pour ne pas nuire aux sujets à conserver. Cette opération, la plus importante sans contredit des coupes d'amélioration, se pratique surtout pendant les premières années de l'existence des massifs. Lorsqu' on la reconnaît utile, on ne doit, sous aucun prétexte, en différer l'exécution, et, nous le répétons, les soins qu'elle exige résident moins dans l'exploitation elle-même que dans le choix des arbres ou des parties d'arbre à supprimer et dans la vidange des produits.

L'opération des éclaircies consiste à couper les tiges les plus faibles et les plus malvenantes, celles qui sont surmontées ou près de l'être et dont la végétation est languissante, enfin les rejets des bois tendres qui se seraient reproduits après les nettoiements. Ces deux coupes peuvent avoir un résultat commun, celui de desserrer les massifs, mais elles diffèrent, en principe, en ce que dans l'une on n'enlève que des sujets dominants, et dans l'autre des sujets dominés. De même que pour les nettoiements, les règles à suivre dans l'exploitation des éclaircies sont données dans le Cours de Culture des bois de 
MM. Lorentz et Parade, et les soins à prendre dans l'abatage ne consistent qu'à éviter, le plus possible, les dégâts que les arbres en tombant peuvent occasionner à ceux qui doivent rester debout.

L'élagage ou la taille d'un arbre consiste à supprimer ou à raccourcir une ou plusieurs de ses branches avec un instrument tranchant. Cette opération s'applique surtout aux arbres réservés dans les taillis composés et, par exception seulement, aux arbres venus en massif de futaie.

L'élagage des arbres de réserve peut avoir pour objet soit de diminuer l'influence de leur couvert sur la végétation du sous-bois, soit d'améliorer leur croissance, de redresser leur forme, ou de prévenir les vices qui pourraient les envahir.

Les arbres à feuillage abondant, comme le hêtre, peuvent causer un dommage considérable au sousbois existant, et même faire obstacle à toute régénération sous leur épais couvert. Il est utile, par conséquent, de les élaguer, en supprimant de ces réserves les branches les plus rapprochées du sol; mais il faut se garder d'exagérer cette opération et ne pas oublier qu'un arbre ne peut prospérer qu'à la condition que le développement de sa cime soit en rapport avec les dimensions de son fut.

Les chênes ont un feuillage beaucoup moins abondant ; leur couvert est léger et ne porte pas un préjudice sensible au sous-bois. L'élagage de ces arbres ne doit donc se faire, en général, qu'en vue d'amé- 
liorer leurs formes ou de prévenir les dangers auxquels ils sont exposés.

Pendant l'été qui suit l'abatage d'une coupe de taillis sous futaie, on voit presque toujours se développer, le long du tronc des chênes réservés, une quantité plus ou moins forte de bourgeons, de feuilles et de petites branches extrêmement vivaces. Ces branches sont dites gourmandes el, si on les laisse persister, elles attireront à elles la plus grande partie de la séve destinée à alimenter la cime. Peu d'années après, quelques branches du sommet, dépourvues de nourriture, mourront, sécheront, se briseront sous l'action du vent, et donneront ainsi naissance aux gouttières, puis aux différents vices qui affectent si fréquemment le tronc des chênes réservés dans les taillis. Au fur et à mesure que le taillis grandira, les branches gourmandes, à commencer par les plus basses, sécheront à leur tour, parce qu'elles seront privées de lumière; elles tomberont, pour la plupart, en laissant dans la tige de petits nœuds qui pourront faire obstacle au débit de l'arbre en bois de fente; en même temps les branches du sommet qui auront conservé leur vitalité reprendront vigueur. Nais, habituellement, quelques-unes des branches gourmandes continueront à végéter, malgré la croissance du taillis, et elles formeront plus tard des branches assez fortes, soit pour déformer la tige, soit pour produire des nœuds qui diminueront la qualité et la valeur du tronc lorsque l'arbre devra être abattu et débité en bois d'œuvre. 
Ces inconvénients sont toujours plus ou moins graves, mais il est facile de les éviter en supprimant les branches gourmandes en temps utile. Le moment le plus favorable pour faire cet émondage est la fin de l'été qui suit l'abatage du taillis. On répète ensuite l'opération une fois ou deux fois, si cela est nécessaire, les années suivantes, et l'on parvient ainsi, par un travail facile et peu coûteux, à prévenir les dangers que nous venons de signaler et à assurer le plus beau développement possible des arbres réservés.

Cet émondage se fait avec une serpe ordinaire, ou avec une serpe en forme de croissant que l'on emmanche au bout d'une perche. Il importe que la section soit faite exactement rez-tronc, qu'elle soit bien nette, et pratiquée de bas en haut. En même temps que l'on procède à l'émondage des branches gourmandes, il est utile de retrancher les branches mortes de la cime. Ces branches doivent aussi être coupées rez-tronc avec un instrument bien tranchant; la plaie qui en résulte doit être parfaitement avivée et recouverte, autant que possible, d'une couche de coaltar. Nous ne parlons pas de l'élagage des branches vives un peu fortes, parce que nous pensons que la suppression de ces branches est plutôt dangereuse q̨u'utile à pratiquer sur les chênes réservés dans les taillis.

- Dans les forêts de sapin et d'épicéa, particulièrement dans celles qui sont soumises au mode du jardinage, on voit souvent, en nombre plus ou moins 
grand, des chicots de branches sèches le long du fut des arbres. Ces chicots persistent quelquefois fort longtemps et, par le fait du grossissement du tronc, ils forment dans la tige des espèces de chevilles de bois mort qui peuvent déprécier notablement la valeur du bois. Quand, par exemple, on débite un de ces arbres en sciages, bon nombre de planches, suivant la direction du débit, sont affectées de nœuds secs et noirs qui proviennent de la section faite dans ces chicots par la scie. Ces nœuds se détachent facilement quand le bois 'se dessèche, et donnent lieu à des trous qui affectent la qualité de la planche jusqu'à en réduire la valeur à la moitié de celle d'une planche nette de nœuds.

La suppression de ces chicots sur des arbres exploitables ou à peu près ne présenterait aucun intérêt, tandis qu'en l'effectuant sur des arbres encore jeunes on ajouterait beaucoup à leur valeur dans l'avenir. Ce système d'élagage est appliqué depuis plusieurs années dans quelques forêts des Vosges appartenant à des propriétaires particuliers; il n'est pas douteux qu'il produise d'excellents résultats, et nous ne pouvons que faire des voux pour le voir propager dans les sapinières de l'Etat et des communes.

Inutile de dire que, dans toutes les opérations d'élagage, il est indispensable que les ouvriers se sèrvent d'échelles pour monter sur les arbres; l'emploi des crampons étant essentiellement nuisible, par les déchirures qu'ils causent à l'écorce et les piqùres qu'ils impriment dans le bois. 
Article IV.

Des bois qui s'exploitent avec la faculté d'écorcer.

Les écorces de plusieurs essences forestières sont ou peuvent être employées au tannage des peaux : telles sont principalement celles des chênes rouvre, pédonculé, tauzin, chevelu, yeuse, liége et kermès; de l'aune, du saule marceau, du bouleau, de l'épicéa et du jeune mélèze. Dans le nord de l'Europe, où le chêne fait défaut, on emploie spécialement les écorces de saule, d'aune et de bouleau, èt c'est à une huile balsamique résineuse que l'on extrait de celle du bouleau par distillation que les cuirs de Russie doivent l'odeur et la couleur qui les caractérisent. L'écorce d'aune est aussi utilisée par les teinturiers et les chapeliers pour teindre en noir; celle de tilleul sert à faire des nattes, des tapis et surtout des cordes très-recherchées pour certains usages, parce qu'elles résistent mieux à l'humidité que celles de chanvre. L'écorce du chêne liége et celle du chêne occidental fournissent des produits dont chacun sait l'usage, et dont l'extraction s'opère suivant des règles qui ont été données dans le Cours de culture des bois de MM. Lorentz et Parade et dans la Flore forestière de M. Mathieu.

A l'exception du liége dont on fait un grand commerce en Provence, dans les Landes, en Corse et en Algérie (1), et des écorces, propres au tannage, des

(1) Le kilogramme de liége en planches vaut, en moyenne, 0 fr. 50 à 
diverses espèces de chêne, ces produits n'ont point d'importance, en France, au point de vue forestier.

Les écorces des chênes occupent le premier rang parmi celles qui peuvent servir à la préparation des cuirs; réduites en farine grossière dans des moulins spéciaux, elles prennent le nom de tan (1).

L'époque la plus favorable pour écorcer le chêne est celle où la séve du printemps se met en mouvement. Cet espace de temps, souvent très-court, est compris entre le moment où les bourgeons commencent à se gonfler et celui où les feuilles vont s'épanouir. Plus tard, l'écorce devient plus adhérente au bois et plus difficile à détacher. On dit de plus qu'elle a moins de qualité que lorsqu'on la récolte pendant les premiers mouvements de la séve. L'écorcement est toujours plus facile par un temps chaud et humide, que par un temps sec. Alors même que la température est élevée et que la végétation est en pleine activité, les vents du Nord et de l'Est apportent toujours un obstacle plus ou moins grand à l'écorcement. Lorsque la végétation est ralentie

Marseille, et c'est par millions de kilogrammes que l'Algérie peut en produire.

(1) L'opération du tannage consiste à stratifier les peaux avec des couches de tan dans des fosses que l'on remplit d'eau. L'eau dissout le taunin qui pénètre peu à peu les peaux, précipite la gélatine qu'elles renferment et forme avec elle un composé insoluble et imputrescible. - Le commerce des écorces à tan a pris en France un grand développement; on évalue à 500 millions de kilogrammes l'écorce employée par les tanneries françaises. L'exportation des écorces à tan présente, en outre, un petit excédant sur leur importation. 
par une longue série de jours froids, comme cela arrive fréquemment dans les climats du Nord et de l'Est de la France, on est obligé de suspendre l'opération de l'écorcement, ou de la mener avec une telle lenteur qu'on ne peut quelquefois la terminer qu'au milieu de l'été, lorsque les arbres sont entièrement couverts de feuilles. Dans ce cas, on est forcé de retarder plus ou moins le terme d'abatage. $\mathrm{Si}$, au contraire, la végétation marche rapidement, on est intéressé à activer le plus possible l'opération de l'écorcement, afin de la terminer dans les meilleures conditions, c'est-à-dire, avant l'épanouissement complet des bourgeons.

Il résulte de ces diverses observations qu'il faut se hâter d'écorcer le chêne, pendant le premier travail de la végétation. Il y va de la qualité de la marchandise et de l'intérêt de celui à qui elle appartient. Or, on comprendra toute l'importance de ces intérêts, lorsqu'on saura que, dans certaines localités où le bois se paye à des prix ordinaires, l'écorce de bonne qualité a quelquefois une valeur égale à celle du bois dont elle provient. Dans les forêts où on pratique l'écorcement, il importe donc d'apporter le moins d'entraves possible à cette exploitation, et d'accorder aux adjudicataires toutes les facilités compatibles avec les conditions qui doivent assurer la conservation et la reproduction des bois.

Pour faciliter l'opération, on autorise ordinairement l'écorcement du bois sur pied. Cette faculté accordée aux ouvriers leur permet de saisir les jours, 
les heures favorables pour hâter l'exécution de la portion la plus importante, la plus difficile et la plus chanceuse de leur travail, l'écorcement de la partie inférieure des tiges. L'écorcement des branches et de la partie supérieure de la tige est toujours plus facile; il se fait après que l'arbre est abattu, mais souvent un ou deux jours après que l'écorce du bas de la tige a été enlevée.

Comme on le voit, cette opération demande à être conduite avec une certaine intelligence, et, quand on la pratique sur une grande échelle, il faut avoir à sa disposition une quantité d'ouvriers suffisante pour pouvoir profiter des moments favorables à l'écorcement.

Le cahier des charges générales fixe au 15 mai le terme d'abatage des bois qui s'exploitent avec faculté d'écorcer; mais, ainsi que nous venons de le dire, on est souvent obligé de proroger ce délai.

On procède à l'écorcement du chêne de plusieurs manières, selon les localités et selon les dimensions des arbres à écorcer. Quand on écorce sur pied, on doit d'abord faire, au bas des arbres et des perches, une entaille circulaire assez profonde pour arriver jusqu'à l'aubier. Cette couptre a pour but d'empêcher que la souche et les racines ne soient dépouillées de leur écorce, dont l'adhérence complète au bois est une condition indispensable de la production des rejets. Les ouvriers fendent ensuite l'écorce en longueur et par lanières, avec la pointe d'une serpe ou 
avec une lame quelconque, et la lèvent avec un outil en fer, en bois dur ou en os, qui a la forme d'une spatule. Cette écorce s'arrache depuis la coupure circulaire au bas du tronc, jusqu'au point le plus élevé où le bûcheron puisse atteindre. On abat ensuite les arbres pour en écorcer les parties supérieures de la tige et les branches. On expose pendant quelque temps les écorces au soleil, pour les faire sécher, puis on les lie en bottes. Il faut se hâter de les mettre à couvert, car si elles étaient exposées à Ia pluie elles perdraient de leur qualité (1).

Dans les taillis sartés des Ardennes, dont les écorces sont si renommées, on procède avec beaucoup de soin à l'extraction de l'écorce. L'ouvrier se sert à cet effet d'un instrument en os qui se compose d'un tibia de cheval taillé en biseau par l'une de ses extrémités, et armé d'une lame courte, forte et bien tranchante à l'autre extrémité (Voir Pl. 1. Fig. 1). Avec cette lame, l'ouvrier fend l'écorce de l'arbre, d'un seul côté, depuis le point le plus élevé qu'il peut atteindre jusqu'à l'entaille circulaire qu'il a d'abord pratiquée au-dessus du collet de la racine. L'ouvrier introduit ensuite le biseau de son instrument entre l'écorce et le bois, et, en le passant alternalivement de chaque côté de la fente, il parvient à détacher entièrement l'écorce sans produire de déchirures. L'écorce ainsi enlevée tout autour de l'arbre, en un seul morceau, se contracte et s'enroule sur elle-même en forme de cylindre creux. On la

(1) MH. Lorentz et Parale, Cours de culture. 
dépose ensuite, pour la faire sécher, sur un lit de perches disposées en plan incliné. Moyennant cette précaution et grâce à la manière dont l'écorce s'enroule sur elle-mème après son extraction, la partie intérieure, celle qui renferme surtout le tannin, ne risque pas d'être lavée par les pluies. On traite de la même manière les écorces du haut de la tige et des branches que l'on n'enlève qu'après l'abatage de l'arbre.

Quand les écorces sont suffisamment séchées, on les nettoie à l'extérieur, avec un couteau ou un racloir, des mousses et autres substances qui pourraient altérer leur qualité et produire des moisissures. C'est alors seulement qu'on les lie en bottes soit pour les mettre en meules, soit pour les transporter et les tenir à couvert sous un hangar, jusqu'au moment où elles seront découpées en menus morceaux et réduites en poudre dans des moulins à tan.

Les écorces qui restent longtemps en meules, ou même sous des hangars mal aérés, sont très-exposées à s'avarier. Souvent elles s'échauffent ou moisissent de manière à perdre presque toute leur qualité. Il faut beaucoup de soin et des frais de manutention assez considérables pour conserver cette marchandise intacte pendant plus de deux ans avant de la livrer aux moulins à tan. A défaut de signes apparents de moisissure ou d'échauffement, on reconnaît que l'écorce est avariée, quand, en la cassant, la couleur de la cassure est d'un jaune brunâtre, ou d'un brun rougeâtre. 
La qualité des écorces est d'autant meilleure que le liber se maintient actif et gorgé de séve sur une plus grande épaisseur ; c'est, en effet, dans la partie vivante de l'écorce que le tannin est le plus abondamment répandu. En général, les écorces de chène les plus riches en tannin sont celles qui proviennent d'arbres jeunes, à végétation rapide, et situés dans un climat chaud. Sous ce rapport, le chêne yeuse accupe le premier rang, le chêne tauzin le second; mais ces deux espèces sont peu répandues en France et ne se rencontrent guère, la première que dans la région Méditerranéenne, la seconde dans les départements du Sud-Ouest. Ce sont les chênes rouvre et pédonculé qui fournissent la presque totalité des écorces qui alimentent nos tanneries, et de ces deux chènes le rouvre est celui qui, à circonstances égales d'âge, de climat et de sol, produit les écorces les plus estimées. Les écorces de bonne qualité doirent être lisses et brillantes à l'intérieur ; leur cassure doit être blanchâtre.

L'écorcement diminue du cinquième au sixième le volume du bois.

Le rendement, de mème que la qualité de l'écorce, varie, pour un mème terrain, avec la situation et l'exposition. Dans les sols qui conservent toujours une certaine fraicheur et par suite leur fertilité, comme les terrains schisteux ou granitiques, c'est aux expositions chaudes que l'on obtient l'écorce la meilleure et le plus fort rendement. On estime ce rendement à une moyenne de 50 à ว̆o killogrammes d'écorce sèche par stère de bois de chauffage. 
Le prix des écorces sur nos principaux marchés varie nécessairement avec la qualité de la marchandise. Le prix moyen est aujourd'hui de 10 à $12 \mathrm{fr}$. les 100 kilog. d'écorce séchée, livrable soit sur les ports d'embarquement, soit dans les gares des chemins de fer. Moitié environ de ce prix est absorbé par les frais de manutention et de transport.

Quoique l'écorce ne soit qu'un produit accessoire des forêts, on voit cependant, par la quantité de matière consommée par nōs tanneries, que ce produit joue un rôle assez important dans l'industrie et le commerce français. Il est bien certain aussi que cette exploitation spéciale augmente d'une façon notable la valeur de certains taillis. Mais on reproche à l'opération de l'écorcement d'exiger des frais de manutention assez considérables, et de faire perdre au propriétaire la valeur de la pousse qui se serait produite si, au lieu d'exploiter après le mouvement de la séve du printemps, les bois avaient été abattus pendant l'hiver.

Il est vrai que l'extraction de l'écorce est, relativement, assez coûteuse, en raison de la rapidité avec laquelle on doit y procéder et de la nécessité d'opérer dans une saison où la main-d'œuvre, dans les campagnes, est plus chère qu'en hiver. Mais, pour ce qui concerne la perte résultant du sacrifice que l'on fait de la pousse du printemps, nous sommes porté à croire (1) que cette perte est compensée par

(1) Voir le remarquable mémoire que M. Bouvart, inspecteur des 
une végétation plus vigoureuse de la pousse d'août.

Quoi qu'il en soit, on a cherché, dans ces derniers temps, à éviter ces inconvénients, en appliquant la vapeur à l'écorcement du chêne. M. Maitre, ancien maître de forges, a imaginé dans ce but un appareil consistant dans une chaudière à lessive surmontée d'une caisse en bois pouvant contenir un demi-stère de rondins. Après avoir été soumis à l'action de la vapeur, pendant 20 ou 30 minutes, le bois s'écorce facilement pourvu qu'il ne soit coupé que depuis quelques jours et qu'il ne soit pas trop desséché.

D'après M. Maitre, les avantages de ce procédé seraient : de pouvoir écorcer en toute saison, même les plus menues branches; de fournir une écorce aussi bonne, sinon meilleure, que celle que l'on obtient par les moyens ordinaires; de procurer une économie d'un tiers au moins sur les frais de façon, pourvu que l'on opère assez en grand.

Les expériences de M. Maitre (1) ont eu lieu en public et ont paru satisfaisantes; mais l'emploi de son procédé ne date que de deux ou trois ans, et nous n'avons pas appris que l'essai en ait été fait ailleurs que dans la contrée du Châtillonnais où il a pris naissance. Nous nous bornerons donc à signaler l'invention de M. Maitre comme un progrès dont nous désirons vivement la réalisation.

forêts, a publié, sur la question de l'écorcement du chène, dans la Revue forestière, $\mathrm{n}^{\text {os }}$ d'octobre, novembre et décembre de 1866.

(1) M. Bouquet de la Grye a rendu compte de ces expériences dans

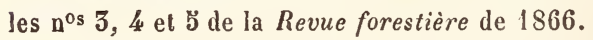




\title{
CHAPITRE DEUXIÈME.
}

\author{
MOde DE DÉbIT, dE MESURAge ET DE VENTE \\ DES BOIS DE CHAUFFAGE.
}

\section{Article I.}

Générali tés.

Les bois de feu ou de chauffage se débitent de quatre manières différentes :

$1^{\circ}$ En bois de quartier, ou bûches refendues;

$2^{\circ}$ En bois de rondin, ou bûches non fendues, moins grosses que les précédentes;

$3^{\circ}$ En fagots, ou faisceaux composés de ramilles, de branches et de quelques rondins ou quartiers;

$4^{\circ}$ En bourrées, ou faisceaux renfermant exclusivement du menu bois.

Dans le commerce, on appelle plus spécialement bois de corde les bois de quartier et de rondin.

\section{Article II.}

\section{Bois de corde.}

\section{§I. Découpe et dressage des bois de corde.}

Les arbres abattus sont débités en bois de feu ou en bois d'œuvre, selon l'usage que l'on veut ou que l'on peut en faire. 
Les arbres, tiges et branches, que l'on convertit en bois de feu, sont débités, partie en bûches de rondin ou de quartier, partie en rames destinées à entrer dans les fagots.

Les bûches de rondin ou de quartier sont découpées à la·hache ou à la scie, selon les localités. L'emploi de la scie est préférable en ce qu'il n'entraìne point de perte dans la découpe des fortes pièces. Les bûches sciées ont aussi une plus belle apparence dans l'empilement et présentent plus d'uniformité dans leur longueur. Les bûches les plus fortes sont ensuite refendues en bois de quartier, avec des coins en fer que l'on chasse dans le bois à coup de masse ou de merlin.

La longueur des bûches de rondin ou de quartier varie généralement entre 1 mètre et 1 mètre 33 centimètres, avec les usages du commerce de chaque localité. Le rondin a ordinairement une grosseur comprise entre 6 et 12 centimètres de diamètre. $\mathrm{Au}$-dessus de 12 centimètres de diamètre, les bûches sont refendues sur place en bois de quartier.

On débite de la même manière le bois que l'on destine à la carbonisation; mais on donne au bois de charbon une longueur moindre, 60 à 80 centimètres environ, et l'on fait souvent entrer dans le bois de charbon des rondins qui n'ont pas plus de 2 à 3 centimètres de diamètre. Ce bois ainsi débité prend le nom de charbonnette.

Les bois de feu façonnés en rondin ou en quartier sont ensuite relevés et empilés soit pêle-mêle 
et sans distinction d'essence ni de grosseur, soit séparément, selon la qualité et les dimensions des bois, par tas plus ou moins considérables qui, dans plusieurs localités forestières, reçoivent le nom de rôles. Mais, en général, les dimensions des rôles sont calculées de manière que chacun d'eux renferme un nombre exact de fois l'unité de mesure des bois de corde.

Quand le bois doit séjourner longtemps sur le parterre des coupes, il importe beaucoup à sa conservation qu'il soit empilé dans des vides dont le terrain soit, autant que possible, plat, uni et sec, et même sur de grosses bûches transversales, ce qui permet la circulation de l'air en dessous des rôles. Les bois empilés sur des places humides s'altèrent bientôt et perdent de leur qualité. Ils se piquent, s'échauffent, se couvrent de moisissures et de champignons plus ou moins promptement selon les essences et, pour une même essence, selon que le bois est couvert ou non de son écorce. On dit alors que le bois est passé, ce qui se reconnaît facilement à la simple inspection des parties les plus voisines de l'écorce. Le bois passé brûle difficilement et sans flamme, il ne donne presque plus de chaleur et, par conséquent, a perdu beaucoup de sa valeur. Parmi les bons bois de feu, le charme et le hêtre s'échauffent assez facilement, le bois de branche surtout, quand ils séjournent plus de quelques mois dans les coupes, en lieu humide. Le chêne résiste 
beaucoup plus longtemps, surtout quand il est écorcé. Le tremble moisit vite et se couvre de champignons. Il est donc très-essentiel d'enlever les bois de feu aussitôt qu'ils sont suffisamment desséchés, c'est-à-dire, pendant l'été qui suit l'abatage.

Quand les bois sont rendus à destination, il convient de les rentrer par un temps sec et, quand on le peut, de les tenir à couvert dans des lieux bien aérés, des hangars, des remises ou des greniers. Ainsi traités, les bois de feu font le meilleur usage possible et peuvent se conserver plus ou moins longtemps, quelquefois pendant plusieurs années, sans s'altérer. Ces précautions doivent ètre prises pour préserver les bois de la vermoulure et de la pourriture, jusqu'au moment où ils seront employés (1).

Les bois pourris et les bois vermoulus, que l'on désigne aussi sous le nom de bois usés, piqués ou passés, ont perdu presque toute leur puissance calorifique. Les bois tendres, les bois jeunes, l'aubier sont plus particulièrement exposés à la vermoulure ; les bois très-vieux, surtout ceux qui ont déjà un commencement d'altération au cœur, sont les plus sujets à la pourriture.

Les bois qui ont été flottés se dessèchent ensuite

(1) La pourriture est due à la présence de principes azotés qui agissent comme ferments sur les substances qui composent le bois. La fermentation se produit sous l'action des alternatives de sécheresse, d'humidité et de chaleur de l'atmosphère.

La vermoulure est due à la présence de larves d'insectes qui creusent des galeries dans le bois, et se nourrissent de la gomme, de la fécule, des sucres, elc., qu'il renferme. 
beaucoup mieux et plus promptement; ils sont moins exposés que les autres à la vermoulure, mais il est certain aussi que le flottage use et altère leur qualité, comme combustibles, lorsqu'il est trop prolongé ou lorsqu'il se fait à plusieurs reprises. - Le hêtre, comme bois de feu, passe très-vite, surtout le rondin de branche. Le bois de quartier se conserve mieux, mais il est toujours à conseiller de l'employer dans l'hiver qui suit l'abatage. - Le charme résiste plus longtemps, lorsqu'il est mis à couvert, surtout quand il est fendu et bien ressuyé. - Quant au chêne, il peut se conserver plusieurs années, quand il est de bonne qualité et convenablement traité.

\$II. - Mode de mesurage et de vente des bois de corde.

Corder le bois, c'est l'empiler sous la forme d'un parallélipipède de dimensions variables. Anciennement les bois de chauffage se vendaient à la corde. Les dimensions et le volume de la corde variaient avec les localités; mais assez généralement on entendait par corde ordinaire un parallélipipède rectangle ayant 8 pieds de couche, 4 pieds d'élévation et 4 pieds de profondeur. La corde comprenait donc 128 pieds cubes.

La corde d'ordonnance des eaux et forêts ford. de 1669) avait 8 pieds de couche, 4 pieds de haut et 3 pieds 6 pouces de profondeur ou de longueur de bûche, ce qui faisait 112 pieds cubes. 
La voie de Paris équivaut à la moitié de la corde d'ordonnance, c'est-à-dire, à 56 pieds cubes.

La corde de port avait 8 pieds de couche et 5 de hauteur, la bûche ayant 3 pieds 6 pouces de longueur. Elle contenait, par conséquent, 140 pieds cubes.

Ces anciennes mesures et bien d'autres sont encore en usage dans une grande partie du commerce des bois de feu, mais on les rapporte au stère qui est l'unité de mesure prescrite par la loi du 4 juillet 1837 pour la vente des bois de feu débités en bûches de quartier ou de rondin.

Le stère est un volume de bois de feu, empilés ou cordés, ayant un mètre de couche, un mètre de hauteur et un mètre de profondeur ou de longueur de bûche. Le stère a, par conséquent, les mêmes dimensions que le mètre cube dont il diffère cependant, comme unité de mesure, en ce que dans le volume du stère on comprend les interstices ou vides qui existent entre les bûches empilées, tandis que le mètre cube exprime un volume plein et sans vides. Mais, comme la longueur des bûches de chauffage varie avec les localités, on est obligé, dans le mesurage de ces bois, de faire varier les deux autres dimensions du mètre cube pour retrouver exactement le stère. Les règlements sur les nouvelles mesures exigent de plus que le bois de feu soit empilé, eu égard à la longueur des bûches, à une hauteur telle que la mesure en stères et en décistères soit exactement indiquée par la longueur en mètres et en 
décimètres de la ligne de couche. Par exemple, on sait qu'en France la longueur de la bûche de bois de feu est assez ordinairement de 42 pouces de notre ancien pied de roi, ou environ 1 mètre 14 centimètres; pour former le stère avec des bûches de cette longueur, on empile le bois sur un mètre de couche et $0^{\mathrm{m}}, 88$ centimètres de hauteur. En anciennes mesures, le stère ou mètre cube vaut 29 pieds cubes et 300 pouces cubes.

Dans le commerce de détail, on vend quelquefois le bois au poids. Les fournitures de bois de feu à certains établissements publics se font assez souvent de cette manière. Mais, pour un même volume, le poids varie avec l'essence, l'âge du bois, la partie de l'arbre dont il provient, et les conditions de sol et de climat dans lesquelles le bois a crû. En Lorraine, le poids moyen d'un stère de bois, empilé suivant les usages du commerce, est d'environ :

$435 \mathrm{k}$. pour le bois de vieux chêne;

$450 \mathrm{k}$. pour le jeune chêne et le hêtre, bois de tige; $500 \mathrm{k}$. pour le rondin de charme;

$533 \mathrm{k}$. pour le quartier de charme.

A Paris, on estime en moyenne le poids d'un stère de bois dur à 400 kil. et le poids d'un stère de bois blanc à 300 kil. (Ann. forest.)

Le tableau suivant fait connaître les densités minima et maxima des principaux bois de France, telles qu'elles ont été déterminées par des expériences faites sur les nombreux échantillons, séchés à l'air libre, des collections de l'École forestière. 


\begin{tabular}{|c|c|c|c|c|c|c|c|c|}
\hline & & & & & & & \multicolumn{2}{|c|}{ DENSITÉ } \\
\hline \multicolumn{7}{|c|}{ ESSENCES. } & Minim & Jaxima \\
\hline Alisier blanc . . & & & - & - & - • & - . & 0,734 & $0,9 \overline{5} 8$ \\
\hline - torminal. & & & - & - & . . & - & 0,817 & 0,989 \\
\hline Aune blanc. . . & . & & - & . & - $\cdot$ & - $\cdot$ & 0,468 & 0,510 \\
\hline - commun. . & • & & - & · & - • & - $\cdot$ & 0,444 & 0,662 \\
\hline Bouleau blanc. . & • & & - & - & - • & - . & 0,517 & 0,771 \\
\hline Cerisier merisier. & • & & - & - & - $\cdot$ & - $\cdot$ & 0,654 & 0,783 \\
\hline Charme commun. & • & & - & - & - $\cdot$ & - $\cdot$ & 0,759 & 0,902 \\
\hline Châtaignier commun & $\mathrm{n}$ & & - & - & - $\cdot$ & - . & 0, อัวิ1 & 0,742 \\
\hline Chêne chevelu. • & • & & - & - & - · & $\cdot$. & 0,855 & 0,925 \\
\hline - liége. • • • & & & - & • & - $\cdot$ & - $\cdot$ & $0,80 \bar{\jmath}$ & 1,029 \\
\hline - occidental. . & • & & . & - & - · & - . & 0,768 & 0,947 \\
\hline - pédonculé. & • & & - & . & .. & . . & $0,65 \overline{3}$ & 0,900 \\
\hline - rouvre. . & • & & - & - & - . & - . & 0,3772 & 1,020 \\
\hline - tauzin.. : & : & & . & - & - . & - $\cdot$ & 0,785 & $0,9 \breve{2}$ \\
\hline - yeuse... & • & & - & . & - . & - $\cdot$ & 0,905 & 1,182 \\
\hline - zeen... & • & & · & . & . & - . & 0,622 & $0,94 \overline{5}$ \\
\hline Erable champêtre & • & & • & - & - . & . . & 0,599 & 0,810 \\
\hline - plane ... & & & - & - & - . & - . & 0,565 & 0.842 \\
\hline - sycomore. & - & & - & - & - . & - . & 0,573 & 0,737 \\
\hline Frêne commun. . & - & & . & - & . . & - : & 0,626 & $0,9 \vec{\jmath} 0$ \\
\hline Hêtre commun. . & - & & - & - & . & - • & 0,686 & 0,907 \\
\hline Micocoulier d'Orient & & & . & - & . . & . . & 0,605 & 0,788 \\
\hline Noyer commun . & & & . & - & - . & - . & 0,579 & 0,800 \\
\hline Olivier d'Europe. . & • & & . & - & - . & - • & $0,8 \overline{36}$ & 1,117 \\
\hline Orme champêtre. & • & & - & - & - . & - . & 0,603 & 0,854 \\
\hline - diffus. . . & - & & - & - & - & - . & 0,554 & 0,676 \\
\hline
\end{tabular}




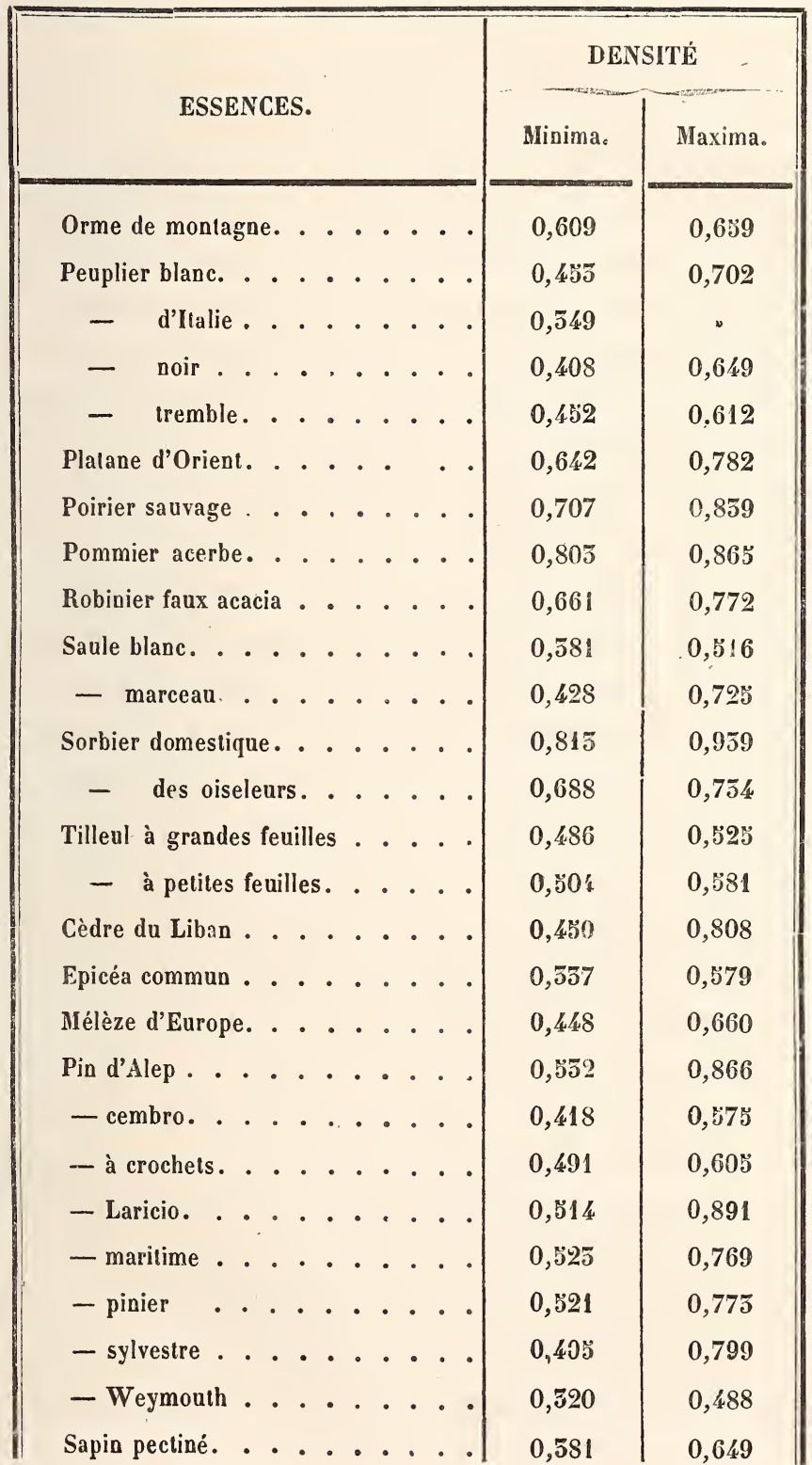


\$ III. - Qualités et défauts des bois de corde.

Les qualités du bois de feu doivent être de brûler facilement, d'une manière égale, sans trop de promptitude ni trop de lenteur, et de fournir, pour un volume donné, la plus grande somme de chaleur.

La puissance calorifique d'un bois est proportionnelle à la quantité de carbone et d'hydrogène libre qu'il renferme. Il est constant que les bois, quels qu'ils soient, ramenés à un degré complet de dessiccation et pour un même poids, renferment des quantités de carbone et d'hydrogène à peu près égales; d'où il suit que, sous des poids égaux, tous les bois ont sensiblement la même puissance calorifique ou, ce qui revient au même, que la puissance calorifique de tous les bois est proportionnelle à leur densité. Pour une même essence, la densité varie avec le climat, la situation, l'exposition et le terrain, avec l'âge de l'arbre et la saison dans laquelle il a été exploité, enfin avec la partie de l'arbre, tige ou branche, aubier ou bois parfait, d'où le bois est extrait. C'est dans les climats chauds que le bois acquiert, en général, la plus grande densité. Chez les feuillus le bois de branche a moins de densité et de caloricité que celui de la tige; le contraire a lieu chez les bois résineux. A circonstances égales, le chêne de taillis a plus de densité, parce qu'il a crû plus vite, que le bois de même âge provenant de futaie, et il est plus estimé que celui-ci. II en est de 
même des réserves sur taillis comparées aux arbres de même essence et de même âge venus en futaie (1). On peut donc mesurer, d'après la densité, la puissance calorifique absolue des bois de feu. Mais la valeur vénale de ces bois se modifie beaucoup par le degré de dessiccation et surtout par la manière dont ils brûlent.

Ainsi les bois qui se dessèchent difficilement sont mauvais combustibles; d'autres ne brûlent qu'à leur surface, parce qu'ils sont très-compactes et ne laissent pas pénétrer dans leur intérieur l'air nécessaire à la combustion du carbone qu'ils renferment. Ces bois laissent beaucoup de charbon, ils durent longtemps dans le foyer, mais ils donnent peu de chaleur. Les bois légers, poreux, brûlent rapidement et avec flamme, ils donnent une chaleur vive mais courte, laissent pénétrer l'air dans leur sein et ne produisent pas de charbon, parce que celui-ci brûle en même temps que les gaz; enfin, il y a des bois qui pétillent et éclatent au feu, ce qui est un inconvénient dans les foyers domestiques.

Ce sont ces avantages et ces inconvénients qui déterminent la valeur relative des bois de feu dans chaque localité et dans les usines où ils doivent être employés.

(1) Ce que nous disons du chêne peut s'affirmer de la même manière pour les essences qui, comme le frêne et le châtaignier, ont la même structure anatomique. Mais les expériences faites jusqu'à ce jour ne permettent pas de conclure de la mème façon pour les essences, comme le hètre et le charme, qui ont une structure différente. 
Les bois piqués, vermoulus, pourris, ou ayant seulement un commencement d'altération, perdent de leur valeur en proportion de la gravité du mal dont ils sont atteints.

Dans le commerce, il y a différents assortiments de bois de corde.

Le bois neuf est le meilleur; c'est celui qui arrive au lieu de consommation par bateau ou par charroi. On distingue aussi le bois neuf du bois vieux en ce que le bois vieux est celui qui a plus d'un an de coupe; il peut être aussi bon que le bois neuf, lorsqu'il a été bien conservé.

Le bois pelard est celui dont l'écorce a été enlevée pour en faire du tan et que l'on met au nombre des bois neufs, lorsqu'il a été transporté par les mêmes moyens. Le bois pelard de chêne brûle mieux que le bois non écorcé de même essence, et que l'on désigne sous le nom de bois gris ou bois couvert. Du reste, le bois pelard et le bois couvert varient extrêmement de qualité avec les localités, avec l'âge ou avec la partie de l'arbre dont ils proviennent, avec les conditions de climat et de sol dans lesquelles le bois a été élevé, et avec les espèces de chêne qui le produisent.

Le bois flotté est celui qui se transporte par train ou à bûches perdues par les rivières; lorsqu'il n'a fait qu'un court trajet par eau, on le considère comme bois à demi-flotté et on l'appelle bois de gravier. Le bois de gravier diffère peu du bois neuf.

Le flottage des bois de feu leur fait éprouver un 
degré d'altération d'autant plus grand qu'ils ont séjourné plus longtemps dans l'eau. Si ces bois ont perdu toute leur écorce, ils sont extrêmement légers quand ils sont secs, ils font une grande flamme en brûlant, se consument très-vite et forment peu de charbon. Les bois usés, piqués ou pourris se consument comme de l'amadou, sans produire ni flamme, ni braise.

On constate que le prix de vente des bois de chauffage sur les principaux marchés de France n'a pas beaucoup varié depuis 20 ans. La consommation des bois de feu en France, tant pour les usines que pour les foyers domestiques, est au moins de 30 millions de stères par an.

Article III.

Faconnage des fagots et bourrées.

Au fur et à mesure que les bûcherons découpent les parties de la tige ou des branches d'un arbre propres à donner des bûches de quartier ou de rondin, ils mettent de côté et rassemblent en tas les menus brins, les rameaux, ramilles et brindilles qui doivent être façonnés en fagots ou en bourrées.

On fagotte de différentes manières, suivant les localités, et selon la qualité et la quantité des produits que l'on fait entrer dans un fagot. Ces procédés différents peuvent se réduire à trois : en forme, sur le chevalet et sous le pied. On assemble le bois des fa- 
gots avec des liens en bois tordus qui reçoivent le nom de harts.

On fait des harts avec toutes les espèces de bois qui se prêtent à la torsion. Les meilleures sont en chêne, charme, coudrier, bouleau, cornouiller, hêtre, sapin, etc. Les harts de sapin et de coudrier sont très-estimées et très-employées dans le flottage, on leur donne alors souvent le nom de rouettes, ou de chapelets.

Les fagots et les bourrées se vendent habituellement au cent, au demi-cent et au quarteron.

Article IV.

Bois à charbon.

\section{S I. - Carbonisation du bois en forêt.}

Les bois que l'on découpe en charbonnette se vendent au stère, comme le bois de corde, et se carbonisent habituellement sur place. Pour opérer la carbonisation en forêt, il y a différents procédés. Celui que l'on pratique le plus généralement en France consiste à réunir et à dresser ces bois en meules qui contiennent chacune au plus 50 stères, souvent 10 ou 12 seulement. On donne à ces meules la forme d'une calotte sphérique ou à peu près. Quand le bois esi dressé, on le recouvre l'une couche de feuilles mortes, de mousses, de gazon, etc., et, par-dessus cette couche, on en fait une autre 
avec du terreau et du poussier de charbon que l'on recueille sur les places où l'on a carbonisé précédemment. Au centre de la meule, on ménage une cheminée que l'on forme avec trois ou quatre piquets plantés en cercle et réunis ensemble avec des harts. On place dans cette cheminée de menus débris de bois sec auxquels on met le feu par le haut. Le feu se communique aux bûches les plus voisines et, de là, se répand dans toute la meule. Quand on croit la meule allumée dans toute la hauteur de la cheminée, on bouche avec des gazons l'ouverture laissée à la partie supérieure. Il ne reste plus alors au charbonnier qu'à surveiller et à diriger le feu, de haut en has, de manière à éviter la combustion entière du bois, tout en opérant sa carbonisation. Quand l'ouvrier voit que la carbonisation est parfaite, il éteint la meule en bouchant tous les trous par lesquels l'air peut pénétrer à travers la couverture, puis il laisse refroidir le charbon, le découvre et le retire. Le temps nécessaire pour cuire une meule de charbon varie de quatre à dix jours, suivant la quantité de bois qu'elle renferme.

On choisit de préférence les terrains secs, unis, plats et abrités pour y établir les meules; les meilleures places sont celles où l'on a fait du charbon aux exploitations précédentes. Elles sont toujours faciles à reconnaître.

La saison la plus favorable à la carbonisation est le mois d'août et de septembre. Il faut opérer autant que possible par un temps sec et calme; le 
vent et la pluie sont des temps contraires à une bonne carbonisation.

Le rendement du bois en charbon varie avec les essences et les qualités de bois, et aussi avec les circonstances atmosphériques qui accompagnent la carbonisation. Le rendement en volume est compris entre 40 et 33 p. 0/0 du volume du bois desséché à l'air libre. Il faut donc deux stères et demi à trois stèrés de bois de charbonnette pour donner un mètre cube ou un kilolitre de charbon. En certains pays on donne précisément ce volume de 2 st. 1/2 à la corde ou moule de charbonnette. - Dans les meilleures conditions, le rendement en poids est d'environ 18 à 22 p. 0/0, jamais il ne dépasse 25 p. $0 / 0$ du poids du bois exposé à l'air libre pendant deux ou trois mois après la coupe.

Le charbon bien fait conserve la forme du bois dont il provient; il est peu cassant, peu gerçuré et très-sonore. On dit qu'il est trop cuit, lorsqu'il perd sa sonorité et qu'il est très-gerçuré; trop peu cuit au contraire, s'il n'est pas noir partout, et si la cassure n'est pas brillante.

Dans ces derniers temps, on avait établi, à proximité de quelques grandes forêts, des usines où l'on carbonisail le bois en vase clos. Nous avons vu fonctionner une de ces usines dans le Bas-Rhin. L'appareil se composait d'un cylindre en tôle pouvant contenir environ 50 stères de bois, et monté sur deux murs en maçonnerie, espacés de manière à 
former un four sous le cylindre; à ce cylindre étaient adaptés des tuyaux qui servaient à l'échappement des gaz provenant de la distillation du bois. Une partie du gaz hydrogène carburé était utilisée pour l'éclairage de l'usine et pour la cuisson du charbon. Les autres produits de la distillation, après avoir parcouru de longs tubes pour se refroidir, venaient se condenser dans des cuves et fournissaient un grand nombre de substances diverses. Les plus importantes étaient l'acide pyroligneux, ou acide acétique impur, que l'on saturait par de la chaux et qu'on livrait au commerce à l'état d'acétate de cette base, le goudron, etc...

Les charbons que nous avons vu faire dans cette usine, étaient beaux, cuits à point, et nous ont paru réunir toutes les qualités recherchées pour les foyers domestiques. Peut-être étaient-ils trop cuits pour l'usage des forges, mais c'est un défaut auquel on aurait pu sans doute remédier, en apportant les soins et les précautions nécessaires dans la cuisson. - 36 heures suffisaient pour la carbonisation de 50 stères de bois.

Pour apprécier le rendement que l'on obtient par ce mode de carbonisation, nous avons fait dans cette usine quelques expériences dont nous allons rendre compte.

2 st. 500 de chêne, rondin fendu et pelé, bien desséché, pesant 873 kilog., ont donné 245 kil. 50 de charbon, soit 28,12 p. $0 / 0$.

2 st. 500 de charme, rondin et petit quartier, 
bien desséché, pesant 866 kil., ont rendu 223 kil. 50 de charbon, ou 25,80 p. $0 / 0$.

2. st. 500 de hêtre, petit quartier, desséché, pesant 1095 kil., ont rendu 280 kil. 5ै de charbon, soit 25,43 p. $0 / 0$.

Le rendement en volume était de 45 à 50 p. $0 / 0$.

Le charbon était bien cuit, luisant, sonore et ne renfermait pas un seul fumeron.

Ce premier pesage a eu lieu lorsqu'on a sorti le charbon de la chaudière, alors qu'il était encore chaud. Mais le poids du charbon augmente quand il est exposé à l'air et refroidi. Nous avons pesé de nouveau ce même charbon, le lendemain, après qu'il eut passé 24 heures sous un hangar bien aéré, et nous avons trouvé que son poids avait augmenté de $3 \mathrm{p} .0 / 0$.

Or, c'est dans ces conditions que le charbon est livré au commerce; il s'ensuit donc que l'on peut considérer le rendement en poids comme étant :

de 28,96 p. $0 / 0$ pour le chêne.

de 26,57 p. $0 / 0$ pour le charme.

de 26,19 p. 0/0 pour le hêtre.

Mais il ne faut pas oublier : $1^{\circ}$ que ce rendement est celui du bois desséché dans une sorte d'étuve et, conséquemment, privé de presque toute l'eau hygrométrique que renferme le bois carbonisé par meules, de sorte que la proportion de carbone y est plus élevée pour un même poids; $2^{\circ}$ que l'on ne tient pas compte du combustible, quel qu'il soit, employé à la dessiccation, ce que l'on fait nécessairement dans 
l'évaluation du rendement des meules. Il en résulte que ces dernières, bien conduites, ne le cèdent point, à cet égard, au système des vases clos. Aussi l'usine dont nous venons de parler n'a-t-elle fonctionné que pendant un an.

\section{\$1I. - Mode de mesurage et de vente du charbon.}

Le charbon de bois est un combustible dont on fait un grand usage dans les foyers industriels et domestiques.

Dans le commerce, le charbon de bois se mesure et se vend ordinairement à l'hectolitre, au poincon, à la verse, à la voie et à la banne.

Le poinçon, ancienne mesure d'ordonnance contenait 240 pintes, mesure de Paris, ou environ 240 litres.

La verse, sorte de corbeille qui, dans certaines contrées, est appelée van, contient environ 37 litres. Mais cette mesure de capacité varie beaucoup avec les localités.

La voie de charbon, mesure de Paris, contient 200 litres. (Ann. forest., 1856, page 137.)

La banne est, comme la verse ou le van, une mesure très-variable. La banne est une sorte de fourgon, formé avec des claies, qui sert surtout à transporter les charbons au lieu de consommation ou sur les ports d'embarquement.

Ces anciennes mesures tendent à disparaître du commerce et sont généralement remplacées par 
l'hectolitre. Dans quelques localités, on cherche aussi à substituer à la vente à l'hectolitre la vente au poids qui est beaucoup plus rationnelle, surtout pour le commerce de détail. En effet, tandis qu'à volume égal, le charbon de bois dur donne plus de chaleur que le charbon de bois tendre, un même poids de charbon donne la même quantité de chaleur, quelle que soit l'essence dont il provient. Il est à remarquer seulement que le charbon de bois tendre brûle et se consume plus vite que le charbon de bois dur. Dans les forges, le charbon de bois dur est surtout estimé pour la fonte du minerai; le charbon de bois plus tendre, de tremble, d'aune, de pin, de sapin, etc., s'emploie avec avantage dans les feux d'affinerie. Le premier se désigne sous le nom de charbon fort, le second sous le nom de charbon doux.

Dans les établissements industriels, on apporte une grande attention à la qualité des charbons qu'oi emploie. Cette qualité s'apprécie d'après la densité du bois et, par conséquent, pour une même essence, elle peut varier avec l'âge des arbres. Le meilleur charbon est fourni par le bois qui touche à sa maturité; c'est ce qui fait que le charbon provenant de bois exploité en taillis, à un âge convenable, est aussi bon que celui qui provient de jeunes futaies. Les bois très-vieux, les bois sur le retour, dépérissants, échauffés, les bois flottés et les bois très-jeunes ne donnent relativement que du charbon de qualité inférieure. La qualité du charbon dépend aussi de 
son degré de cuisson, de la saison dans laquelle le bois a été abattu et de son degré de dessiccation au moment de la carbonisation. Le charbon cuit à point, donne plus de chaleur que le charbon trop cuit ou pas assez cuit. Le bois coupé dans la saison morte donne plus de chaleur et un meilleur charbon que le bois coupé en temps de séve. Le bois que l'on carbonise après quelques mois de coupe, et lorsqu'il ne renferme plus que 20 à 25 p. 0/0 d'eau hygrométrique, donne un charbon meilleur que celui que l'on obtient du bois vert ou du bois très-desséché.

Le charbon bien fait varie de poids avec les essences et la qualité du bois. En Lorraine, le charbon de bois dur mêlé pèse en moyenne 22 à 25 kil. l'hectolitre.

Dans le transport des charbons, on doit éviter, autant que possible, les secousses, les cahots de voiture, les transbordements, etc., qui ont toujours pour résultat de briser le charbon, ce qui détermine un déchet dans la quantité et aussi, dit-on, dans la qualité du combustible. Rendu à destination, le charbon doit être mis en lieu sec et aéré, et surtout préservé de la pluie.

Le charbon de bois dur n'a pas beaucoup varié de prix sur le marché de Paris. Il se cote aujourd'hui, comme il y a 20 ans, au prix moyen de 4 fr. l'hectolitre. Mais ce produit a beaucoup diminué d'importance, dans quelques centres forestiers de France, depuis que l'on a successivement introduit l'usage de la houille et du coke dans la plupart des établis- 
42 DES FAGTEURS DE CONVERSION DU MĖTRE CUBE PLEIN sements métallurgiques qui, autrefois, ne consommaient que du charbon de bois. Cela se comprend, puisqu'il ne faut pas moins de 6 à 8 kilolitres de charbon de bois pour réduire le minerai de fer capable de fournir une tonne ou 1000 kilogrammes de fonte, et 5 à 6 kilolitres environ pour affiner, c'est-à-dire, pour transformer en fer la même quantité de fonte.

\section{Article V.}

Des facteurs de conversion du mètre cube plein au volume empilé en bois de feu.

D'après la manière dont on mesure les bois de corde, on conçoit que la masse réelle de bois contenu dans un stère est très-variable et qu'elle est en raison inverse des vides ou interstices que laissent entre elles les bûches empilées. Il en est de même des fagots et des bourrées qui, à dimensions égales, renferment souvent des volumes de bois très-différents.

Le volume réel d'un stère de bois empilé est plus ou moins fort, poar une même essence, selon que les bûches sont plus ou moins grosses, et que le bois est plus ou moins droit et uni, ou tortueux et noueux. Ce volume varie encore avec la longueur des bûches ; il est plus fort lorsque les bûches sont plus courtes, parce qu'alors le bois se range mieux dans l'empilement. 
Il y a deux manières principales de déterminer le volume réel du bois contenu dans un stère empilé : $1^{\circ}$ En plongeant le bois dans une cuve en partie remplie d'eau, et en mesurant le volume d'eau déplacée; $2^{\circ}$ en cubant, par les procédés de la géométrie, chacune des bûches comprises dans le stère empilé.

De ces deux procédés, le dernier est le moins exact, mais il est plus expéditif que le premier. Aussi l'emploie-t-on de préférence dans les expériences que l'on fait en forêt. Il consiste à cuber chaque bûche comme un cylindre régulier de même longueur et ayant pour base le cercle du milieu. Ia somme de tous ces volumes donne la masse réelle du bois compris dans un stère. - Si ensuite on prend le rapport du volume empilé au mètre cube plein, on a le facteur par lequel on doit multiplier un volume plein pour obtenir le rendement de ce volume en stères empilés de chauffage. Ce facteur varie, pour une même essence, a vec les circonstances que nous avons énoncées plus haut; il est toujours plus grand que l'unité, et plus il est faible moins il y a de vides dans l'empilement; on l'appelle facteur d'empilage.

Dans la pratique forestière, les estimations de bois sur pied se font au mètre cube ou au stère; dans le premier cas, le facteur d'empilage sert à calculer le rendement du volume réel en bois de corde; dans le second cas, le rapport inverse sert à déterminer le volume réel du bois estimé. On emploie encore les facteurs d'empilage dans d'autres circonstances dont 


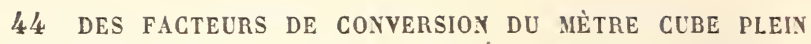

nous parlerons plus tard. Dans tous les cas, pour que les facteurs puissent être employés en toute sécurité dans les calculs de cubage et d'estimation, il faut qu'ils soient le résultat d'expériences nombreuses et faites avec soin, sur des bois de même essence, de mêmes formes et de mêmes dimensions que ceux auxquels ces coefficients s'appliqueront. Aussi, dans ces expériences, sépare-t-on les bois de branches qui sont toujours plus ou moins noueux, tortueux, contournés, des bois de même essence qui proviennent de la tige et qui sont d'ordinaire plus droits et plus unis. On fait également une distinclion entre les bois de tige, selon la grosseur des bûches, parce que les vides qui résultent de l'empilement de ces bûches refendues en bois de quartier, varient avec le nombre des quartiers ou morceaux qu'on en tire. On obtient ainsi, par des moyennes d'expérience, des facteurs particuliers pour chaque essence et, pour la même essence, des facteurs d'empilage des branches et de la tige des arbres.

Lorsqu'on veut rechercher les facteurs d'empilage par ce procédé, la chose importante évidemment et la plus délicate, c'est de déterminer bien exactement le diamètre ou la circonférence au milieu de chaque bûche à cuber. La circonférence se prend avec un ruban, et c'est la manière la plus sûre et la plus exacte de mesurer la base; mais elle exige beaucoup de temps et de soin, et c'est souvent un inconvénient, Le mesurage des diamètres est plus expéditif, et lorsqu'on opère sur une assez grande échelle, on 
peut compter qu'il s'établira, dans les erreurs du mesurage, des compensations suffisantes pour que le volume total des bûches d'expérience n'en soit pas affecté.

Le mesurage du diamètre se fail avec le compas forestier. Cet instrument, d'un usage très-répandu dans la pratique des estimations, se compose d'une règle graduée portant à angle droit deux autres règles plus petites dont l'une est mobile le long de la grande règle et l'autre est fixée à son extrémité. La grande règle est divisée en centimètres à partir de la petite règle fixe; on lui donne ordinairement un mètre de longueur. Les deux autres règles n'ont ordinairement que 60 centimètres de longueur. On prend la précaution de construire l'instrument avec du bois bien sec et qui se déjette le moins possible sous l'influence de la chaleur et de l'humidité (1).

Nous donnerons, à la fin de cet article, un tableau faisant coinnaitre le rendement que l'on obtiendrait,

(1) Des expériences très-nombreuses ont été failes en France, notamment par les Agents des commissions d'aménagement, pour déterminer le rolume réel $d n$ bois compris dans un stère empilé de chauffage. Les forestiers allemands se sont également occupés de cette question et successirement MII. de Burgdorff, Hartig et de Werneck ont publié les résultats de leurs recherches dans des ouvrages dont on peut lire la traduction dans le Dictionnaire des forêts de Baudrillart. Ces résultats sont, comme on devait s'y attendre, très-divergents, et cette divergence même indique assez que les expériences de celte nature doivent être faites dans la forêt ou la localité où l'on a à employer les facteurs d'em. pilage, surtout si l'on reut remarquer que ces facteurs dépendent autant de la manière dont les ouvriers débitent et empilent le bois de corde, que des autres causes déjà signalées. 
46 Des facteurs de CONVERSION du mĖtre cube, etc.

en stères empilés de chauffage, d'une bille de bois de la longueur de 1 mètre, pour un facteur d'empilage variant de 1,40 à 1,70.

Lorsqu'on veut obtenir le volume du bois compris dans un fagot, on le plonge dans une cuve en partie remplie d'eau, et on mesure le volume du liquide déplacé, en enlevant cette eau avec un vase contenant exactement le litre, jusqu'à ce que le niveau soit au point où il se trouvait lorsqu'on y a immergé le fagot. Dans la pratique forestière, cette expérience sert à déterminer le rapport entre le mètre cube plein et le volume du bois compris dans un cent de fagots. A cet effet, on détermine par des pesées le poids moyen d'un fagot; on pèse ensuite un fagot dont on obtient le volume comme précédemment, puis on calcule le volume du cent de fagots par une proportion.

Connaissant le volume du bois compris dans un cent de fagols, on obtient facilement le rapport du mètre cube plein à ce volume. Ce rapport s'appelle le coefficient ou facteur des fagots. 


\begin{tabular}{|c|c|c|c|c|c|c|c|}
\hline \multirow{2}{*}{$\begin{array}{l}\text { DIAMTE- } \\
\text { TRE. }\end{array}$} & \multicolumn{7}{|c|}{$\begin{array}{l}\text { VOLUME EN STERES EMPILES DE CHAUFFAGE } \\
\text { pour un mètre de longueur, et pour un facteur d'empilage égal à }\end{array}$} \\
\hline & 1,40 & 1,45 & 1,50 & 1,55 & 1,60 & 1,65 & 1,70 \\
\hline cent. & st. 0,028 & st. 0,029 & $\begin{array}{l}\text { st. } \\
0,030\end{array}$ & $\begin{array}{l}\text { st. } \\
0,031\end{array}$ & st. 0,032 & $\begin{array}{l}\text { sl. } \\
0,053\end{array}$ & $\begin{array}{l}\text { st. } \\
0,05 \%\end{array}$ \\
\hline 18 & 0,05 วั & 0,056 & 0,057 & 0,059 & 0,040 & 0,041 & 0,042 \\
\hline 20 & 0,043 & 0,045 & 0,046 & 0,048 & 0,050 & 0,051 & 0,0303 \\
\hline 22 & 0,053 & 0,055 & 0,057 & 0,059 & 0,061 & 0,063 & 0,063 \\
\hline 24 & 0,063 & 0,065 & 0,067 & 0,070 & 0,072 & 0,074 & 0,076 \\
\hline 26 & 0,074 & 0,077 & 0,079 & 0,082 & 0,085 & 0,087 & 0,090 \\
\hline 28 & 0,087 & 0,090 & 0,093 & 0,096 & 0,099 & 0,102 & 0,103 \\
\hline 30 & 0,099 & 0,103 & 0,106 & 0,110 & 0,114 & 0,117 & 0,121 \\
\hline 32 & 0,112 & 0,116 & 0,120 & 0,124 & 0,128 & 0,152 & 0,156 \\
\hline 34 & 0,127 & 0,132 & 0,156 & 0,141 & 0,146 & 0,1500 & 0,153 \\
\hline 36 & 0,143 & 0,148 & 0,153 & 0,1 ร8 & 0,163 & 0,168 & 0,175 \\
\hline 38 & 0,158 & 0,164 & 0,169 & 0,17 Ј & 0,181 & 0,186 & 0,192 \\
\hline 40 & 0,176 & 0,183 & 0,189 & 0,195 & 0,202 & 0,208 & 0,214 \\
\hline 48 & 0,193 & 0,200 & 0,207 & 0,214 & 0,221 & 0,228 & 0,235 \\
\hline 4 & 0,213 & 0,220 & 0,228 & 0,235 & 0,243 & 0,251 & 0,258 \\
\hline 46 & 0,232 & 0,241 & 0,249 & 0,277 & 0,266 & 0,274 & 0,282 \\
\hline 48 & 0,253 & 0,262 & 0,271 & 0,281 & 0,290 & 0,299 & 0,308 \\
\hline 50 & 0,274 & 0,284 & 0,294 & 0,504 & 0,314 & 0,523 & 0,353 \\
\hline 52 & 0,297 & 0,507 & 0,318 & 0,329 & $0, \overline{35} 9$ & 0,350 & 0,360 \\
\hline 54 & 0,521 & 0,352 & 0,343 & $0,35 \%$ & 0,366 & 0,578 & 0,589 \\
\hline 56 & 0,544 & 0,357 & 0,369 & 0,581 & 0,594 & 0,406 & 0,418 \\
\hline 58 & 0,570 & 0,383 & 0,596 & 0,409 & 0,422 & 0,436 & 0,449 \\
\hline 60 & 0,596 & 0,410 & 0,424 & 0,439 & 0,453 & 0,467 & 0,481 \\
\hline 62 & 0,423 & 0,438 & 0,453 & 0,468 & 0,483 & 0,498 & 0, อั \\
\hline
\end{tabular}




\begin{tabular}{|c|c|c|c|c|c|c|c|}
\hline \multirow{2}{*}{$\begin{array}{l}\text { DIAME'- } \\
\text { TRE. }\end{array}$} & \multicolumn{7}{|c|}{$\begin{array}{l}\text { VOLUME EN STERES EMPILES DE CHAUFFAGE. } \\
\text { pour un mètre de longueur, et pour un facteur d'empilage égal à }\end{array}$} \\
\hline & 1,40 & $1,4 y$ & 1,50 & 1,55 & 1,60 & 1,65 & 1,70 \\
\hline $\begin{array}{r}\text { cent. } \\
\text { G4 }\end{array}$ & 0,431 & 0,467 & 0,483 & st. 0,499 & st. 0,315 & st. 0,551 & st. 0,547 \\
\hline 66 & 0,479 & 0,496 & 0,513 & 0,550 & 0,5447 & 0,504 & $0, \circlearrowright \$ 1$ \\
\hline 68 & $0, \breve{08}$ & 0,526 & $0, \breve{4} 44$ & $0, \check{6} 63$ & 0,381 & 0,599 & 0,617 \\
\hline 80 & $0,5 \breve{3} ?$ & 0, อัอ & 0,577 & C, 597 & 0,616 & 0,635 & 0,654 \\
\hline 22 & 0,570 & 0,590 & 0,610 & 0,631 & 0,651 & 0,672 & 0,692 \\
\hline 8. & 0,602 & 0,625 & $0,64 \%$ & 0,666 & 0,688 & 0,709 & 0,751 \\
\hline 86 & 0,636 & 0,658 & 0,681 & 0,704 & 0,726 & 0,749 & 0,772 \\
\hline 28 & 0,669 & 0,693 & 0,717 & 0,741 & 0,765 & 0,789 & 0,813 \\
\hline 80 & 0,704 & 0,729 & 0,734 & 0,780 & 0,805 & 0,830 & 0,835 \\
\hline 82 & 0,739 & 0,766 & 0,792 & 0,818 & 0,843 & 0,871 & 0,898 \\
\hline 81 & 0,776 & 0,8005 & 0,851 & 0,859 & 0,886 & 0,914 & 0,942 \\
\hline 86 & 0,813 & 0,842 & 0,871 & 0,901 & 0,930 & 0,9 วั9 & 0,988 \\
\hline 88 & 0,831 & 0,882 & 0,912 & 0,942 & 0,973 & 1,005 & 1,053 \\
\hline 90 & 0,890 & 0,922 & 0,954 & 0,986 & 1,018 & 1,049 & 1,081 \\
\hline 22 & 0,951 & 0,966 & 0,997 & 1,051 & 1,064 & 1,097 & 1,130 \\
\hline 94 & 0,972 & 1,006 & 1,041 & 1,076 & 1,110 & 1,145 & 1,180 \\
\hline 96 & 1,014 & 1,050 & 1,086 & 1,122 & 1,158 & 1,195 & $1,2 \overline{5} 1$ \\
\hline 98 & 1,056 & 1,093 & 1,131 & 1,169 & 1,206 & 1,244 & 1,282 \\
\hline 100 & 1,099 & 1,158 & 1,177 & 1,217 & 1,256 & $1,29 \%$ & 1,334 \\
\hline 102 & 1,144 & 1,185 & 1,225 & 1,266 & 1,307 & 1,348 & $1, \overline{3} 89$ \\
\hline 104. & 1,189 & 1,231 & 1,273 & 1,516 & 1,358 & 1,401 & 1,445 \\
\hline 106 & 1,256 & 1,279 & 1,325 & $1, \overline{5} 67$ & 1,411 & 1,455 & 1,499 \\
\hline 108 & 1,282 & 1,328 & 1,574 & 1,420 & 1,466 & 1,511 & 1,557 \\
\hline 110 & 1,330 & 1,377 & 1,425 & 1,472 & 1,520 & 1,567 & 1,015 \\
\hline
\end{tabular}




\section{CHAPITRE TROISIÈME.}

MODE DE DÉBIT, DE MESURAGE ET DE VENTE

DES BOIS D'OEUVRE.

Article I.

Gén éralités.

Sous la dénomination de bois d'cuvre, on comprend les bois propres à tous les emplois autres que le chauffage. Ils se divisent en :

Bois de service et Bois de travail.

Les bois de service sont ceux qui servent aux constructions civiles et navales. Ces bois s'appellent aussi bois de construction, bois de charpente, et bois de marnage ou de marronnage, quand ils désignent les bois à bâtir auxquels les usagers ont droit dans certaines forêts.

Les bois de travail ou d'industrie comprennent les bois employés par les différents métiers tels que la menuiserie, l'ébénisterie, le charronnage, la tonnellerie, la fabrication des sabots, elc., etc.

Les bois employés de préférence dans les grandes constructions civiles sont ceux du chêne, du chàtaignier, du pin sylvestre et du sapin.

Dans les constructions civiles ordinaires ou communes, on emploie, comme charpente, le chêne, le 
châtaignier, le sapin, l'épicéa, le pin sylvestre, l'orme, le sorbier domestique, le merisier commun et le tremble; tous les peupliers sont très-recherchés, à cause de leur légèreté, pour certains emplois comme cloisons, poutrelles, etc.

Dans les constructions souterraines, on emploie pour pilotis, conduites d'eau, corps de pompe, étancons de mine, etc., le chêne, le pin, l'aune, l'orme, le sapin, le hêtre, le bouleau et le charme.

Les bois de service destinés aux constructions navales se désignent, d'une manière générale, sous le nom de bois de marine. Les pièces de marine recoivent en outre différents noms, selon leurs dimensions et la forme qu'elles affectent.

Parmi les bois de travail ou d'industrie qui se débitent dans les coupes, on distingue spécialement les bois de sciage et les bois de fente. Les premiers sont ceux que l'on débite à la scie pour les transformer en planches de différentes dimensions. Les seconds sont ceux dont l'emploi exige le procédé de la fente ; tels sont les douves de tonneaux, de cuves, etc., les échalas, les lattes, les cerches ou planchettes trèsminces dont on fait les bordures des tamis, des boisseaux et autres mesures, etc. C'est encore avec du bois de fente que l'on fait des panneaux de soufflet, des pelles à four et autres, des attelles de collier, des bâts ou arçons de selle, des rames et des gournables.

On appelle arbres en grume, ou pièces de bois en grume, les morceaux bruts, recouverts ou dépouillés de leur écorce, qui doivent être employés comme bois d'œurre. 
MODE DE DÉbit, DE CUBAGE ET DE Vente DES Bols.

L'équarrissage est l'opération par laquelle on réduit les bois en grume avec la hache et l'erminette de manière à leur donner la forme d'un parallélipipède droit, rectangle, ou oblique. En général, on laisse en grume les pièces qui doivent être vendues et livrées sur le parterre des coupes, tandis que souvent on équarrit sur place les bois de construction ou de travail qui doivent être transportés et livrés hors de la coupe, afin de diminuer les frais de transport.

\section{Article II.}

Mode de débit, de cubage et de vente des bois de service.

\section{§I. - Débit des bois de service.}

Les bois que l'on débite le plus ordinairement en pièces de charpente dans nos forêts sont ceux du chêne, du sapin, de l'épicéa et du pin sylvestre.

Les bois de chêne qui sont destinés à faire un long trajet jusqu'au lieu de vente ou de consommation, sont ordinairement équarris sur place, afin de diminuer les frais de transport; tandis qu'on livre habituellement en grume les bois résineux tels que le sapin et l'épicéa, dont le poids est moins grand pour un même volume, et qui d'ailleurs peuvent être mis en œuvre sans être équarris, parce qu'ils n'ont pas d'aubier.

On ne donne pas de nom particulier aux charpentes de chêne dans les coupes; on se borne sim- 
plement à les distinguer en petites et en grosses charpentes, suivant qu'elies proviennent d'arbres qui ont moins ou plus de 1 mètre 30 centimètres de tour au milieu. Cette circonférence correspond à un équarrissage grossier de $0^{\mathrm{m}}, 32$ à $0^{\mathrm{m}}, 33$ (un pied). Mais pour qu'une pièce de chêne soit classée comme grosse charpente, il faut aussi qu'elle ait au moins 6 mètres de longueur et qu'elle soit assez droite (1).

Les charpentes de sapin et d'épicéa reçoivent différents noms, selon les localités, et selon leurs dimensions en diamètre et en longueur.

Dans les Vosges, on appelle :

Chevron, une pièce ayant au moins 5 mètres de longueur et $0^{\mathrm{m}}, 20$ à $0^{\mathrm{m}}, 25$ de diamètre à la base;

Panne simple, une pièce de petite charpente ayant au moins 12 mètres de longueur et $0^{\mathrm{m}}, 30$ à $0^{\mathrm{m}}, 3 \breve{ }$ de diamètre à la base ;

Panne double, une pièce de charpente moyenne ayant au moins 12 mètres de longueur et $0^{\mathrm{m}}, 40$ environ de diamètre à la hase;

Recharge, une pièce de grosse charpente, quelle qu'en soit la longueur, mesurant $0^{\mathrm{m}}, 30$ à $0^{\mathrm{m}}, 34$ (11 à 12 pouces) d'équarrissage au milieu;

(1) On ne connaît que très-imparfaitement la quantité et la valeur des bois de charpente de chêne que l'on produit et que l'on consomme en France. Mais on sait qu'il faut annuellement 40000 mètres cubes de bois équarris ou environ 80000 mètres cubes de chêne en grume pour l'entretien de la marine militaire. 
Poutre ou Sommier, toute pièce ayant un équarrissage plus fort.

Les bois de petite et de moyenne charpente, dont les dimensions sont trop faibles pour qu'on puisse en faire des planches ordinaires, n'ont qu'une valeur très-inférieure à celle du bois de sciage. La dimension minima de la tronce de sciage ordinaire est d'environ $0^{\mathrm{m}}, 38$ de diamètre au petit bout.

- Dans le Jura, on débite les charpentes de sapin ou d'épicéa de deux manières :

$1^{\circ}$ En Bois ronds, dont le pied seul est équarri à huit pans, sur une longueur de 4 à 5 mètres au plus ;

$2^{\circ}$ En Pièces, qui sont équarries à quatre pans sur toute leur longueur, d'où résulte un déchet évalué en moyenne à $34 \mathrm{p}$. $0 / 0$ du volume en grume.

Ces deux catégories de charpente se classent en outre d'après leurs dimensions :

En Gros bois, qui comprennent les arbres mesurant au moins $0^{\mathrm{m}}, 70$ de diamètre à la base et qui donnent les bois ronds et les grosses pièces;

En Bois moyens, qui comprennent les arbres mesurant de $0^{\mathrm{m}}, 6$ s̆ à $0^{\mathrm{m}}, 55$ de diamètre à la base;

En Petits bois, qui comprennent les arbres mesurant de $0^{\mathrm{m}}, 50$ à $0^{\mathrm{m}}, 20$ de diamètre à la base.

Les Gros bois ont une valeur double, en général, de celle des Petits bois. Celle des gros bois est aujourd'hui d'environ 20 à 25 fr. le mètre cube sur pied, dans les forêts des Vosges et du Jura où l'on 
peut aborder par des voies de vidange faciles et bien organisées (1).

On peut comprendre aussi parmi les bois de service ceux que l'on emploie comme traverses dans la construction des chemins de fer. Les traverses se débitent et se façonnent à la scie dans les coupes en exploitation. Les meilleures sont en chêne; mais on en fait aussi avec d'autres bois, tels que le hêtre et le pin sylvestre, que l'on pénètre ordinairement d'une substance qui les rend plus durables. La tolérance admise assez généralement dans les fournitures de traverses permet d'employer pour cet usage du bois d'une qualité souvent médiocre et d'une forme peu régulière. C'est donc un moyen de tirer un bon parti de certaines pièces qui auraient été peu propres au sciage et aux constructions. Le débit des bois en traverses a une importance déjà grande et qui ne fera que s'accroître dans l'avenir.

On fait des traverses de deux dimensions : les plus fortes, dites traverses de joint, doivent avoir $2^{\mathrm{m}}, 50$ à $2^{\mathrm{m}}, 60$ de longueur, sur $0^{\mathrm{m}}, 30$ à $0^{\mathrm{m}}, 32 \mathrm{de}$ largeur et $0^{\mathrm{m}}, 13$ à $0^{\mathrm{m}}, 1 \mathrm{~b}$ d'épaisseur; les plus faibles, dites traverses intermédiaires, ont également $2^{\mathrm{m}}, 50$ à $2^{\mathrm{m}}, 60$ de longueur, sur $0^{\mathrm{m}}, 21$ à $0^{\mathrm{m}}, 26 \mathrm{de}$ largeur et $0^{\mathrm{m}}, 12$ à $0^{\mathrm{m}}, 13$ d'épaisseur.

On estime qu'il faut de 120 à 130 mètres cubes

(1) La consommation du bois de sapin s'accroît, en France, avec une grande rapidité. En 1865, nous avons reçu de l'étranger plus de 400.000 mètres cubes de bois de charpente, valant plus de 20.000 .000 de francs, el dont les $7 / 10^{e}$ au moins de la Péninsule Scandinave. 
de bois rond pour donner 100 mètres cubes de traverses façonnées, ou environ 1234 pièces dont $1 / 6$ en traverses de joint et $5 / 6$ en traverses intermédiaires. Un mètre cube de bois en grume fournit donc, en moyenne, 10 traverses assorties, et le déchet dû à ce genre de débit est à peu près de 20 p. $0 / 0$ pour le chêne, $30 \mathrm{p}$. $0 / 0$ pour le hêtre.

Il faut environ 1250 traverses assorties pour la construction d'un kilomètre de chemin de fer, y compris les voies d'évitement et de garage. Or, le réseau français comprend aujourd'hui plus de 15000 kilomètres dont la moitié à double voie, ce qui fait en tout 22500 kilomètres, dans la construction desquels il est entré, en nombres ronds, 28.000.000 de traverses assorties, ou 2.800.000 mètres cubes de bois en grume.

Les traverses de chêne durent de 10 à 15 ans; celles en hêtre injecté, 9 à 10 ans; soit en moyenne 12 ans. L'entretien seul de nos voies ferrées actuelles exigerait donc une fourniture annuelle de 200.000 mètres cubes au moins, fourniture qui s'accroîtra beaucoup dans l'avenir, si on ne parvient pas à remplacer le bois par quelqu'autre matière dans la construction des chemins de fer.

Parmi les boị de service on peut encore ranger les poteaux télégraphiques, les étais ou étançons de minne et les bois de chêne, de frêne, d'orme, de charme, de sapin et de pin sylvestre que l'artillerie emploie dans les arsenaux. Les poteaux télégraphi- 
ques se font surtout en pin et en sapin, mais aussi en autres bois (1). Les étais ou étançons de mines servent à la construction des galeries dans les houillières, les minières, etc. Ce sont des perches de dimensions variables et de toutes essences, qui peuvent être fournies en grande partie par les taillis. Ce genre de débit, déjà important aujourd'hui dans quelques départements, paraitt appelé à prendre une extension de plus en plus grande, en raison du développement des exploitations houillières et du transport facile de ces bois par les rivières, les canaux et les chemins de fer (2).

S II. - Mode de mesurage, de cubage et de vente des bois ronds, ou en grume.

Pour évaluer le volume entier d'une pièce de bois ronde, avec ou sans écorce, on la considère ordinairement comme un cylindre de hauteur égale à la longueur de la pièce, et ayant pour base la surface du cercle mesuré au milieu, ou une moyenne entre les cercles mesurés au petit et au gros bout. On obtient ensuite le cube de la pièce par un calcul que tout le

(1) Les poteaux télégraphiques ont, en moyenne, $0^{\mathrm{m}}, 20$ de diamètre au milieu sur 8 mètres de longueur, ce qui représente un cube do $0^{\mathrm{m} \cdot c \cdot, 250}$. Injectés ou non, ils durent au plus 10 ans. Nous avons aujourd'hui en France plus de $\mathbf{3 0 . 0 0 0}$ kilomètres de lignes télégraphiques qui, à raison de 13 poteaux par kilomètre, en comprennent environ 450.000. Leur entretien seul exige donc annuellement, dès aujourd'hui, plus de 12000 mètres cubes de bois.

(2) Quels qu'ils soient, ces bois durent très-peu dans l'air chaud et humide des mines. 
monde connaît, ou bien, ce qui est plus commode et plus expéditif, à l'aide d'un tarif qui donne les volumes cylindriques de tous les arbres dont on connaît la longueur et la circonférence moyenne, ou la longueur et le diamètre moyen. La longueur de la pièce et la circonférence se mesurent avec un ruban gradué ; le mesurage du diamètre se fait avec le compas forestier.

Le résultat du cubage effectué par ces deux procédés s'exprime en mètres et en décimètres cubes. Mais, dans le commerce, on exprime assez généralement ce résultat en solives.

La solive est une fraction du mètre cube. On distingue l'ancienne et la nouvelle solive.

La solive ancienne se considérait sous deux points de vue : $1^{0}$ comme pièce de charpente : c'était un prisme rectangulaire droit de 12 pieds de haut et de 6 pouces d'équarrissage valant, par conséquent, trois pieds cubes; $2^{\circ}$ comme unité de volume : c'était, sous une forme quelconque, l'équivalent de trois pieds cubes, ou en mètres cubes $0^{\text {m.c. }}, 102830$, quand le pied employé était le pied de roi, qui avait une longueur de $0^{\mathrm{m}}, 32484$.

La nouvelle solive n'est autre chose que le décistère ou la dixième partie du mètre cube; elle équivaut, par conséquent, à 100 décimètres cubes. Elle diffère peu, comme on voit, de la solive ancienne; 1000 solives anciennes équivalent à 1028 solives nouvelles (1).

(1) Dans les très-nombreuses localités où le commerce des bois n’a 
Nous venons de dire comment on évalue le volume entier des bois ronds. En terme de cubage, on désigne sous le nom de volume en grume, le volume cylindrique ou entier des bois ronds, qu'ils soient ou non recouverts de leur écorce. Mais on n'emploie guère les bois sous cette forme; le plus souvent on les équarrit plus ou moins fort avant de les mettre en œuvre, soit pour enlever l'aubier en tout ou en partie, soit pour tout autre motif. De là est venu l'usage, presque partout admis dans le commerce, de cuber les bois d'œurre en grume, comme si les pièces étaient équarries.

Il y a trois manières principales de cuber les bois ronds comme s'ils étaient équarris, savoir :

$1^{\circ}$ Au quart sans déduction;

$2^{\circ}$ Au cinquième déduit ;

$3^{\circ}$ Au sixième déduit .

Le cubage d'un tronc d'arbre ou d'une pièce de bois au quart sans déduction consiste à prendre le quart de la circonférence mesurée au milieu de la pièce, ou le quart de la circonférence moyenne entre les deux circonférences mesurées au petit et

pas encore adopté les mesures du système métrique, on attribue à la solive des valeurs très-variables de province à province, et même de ville à ville dans une même contrée. C'est ainsi que dans certains pays la solive métrique, qui ne devrait ètre autre que le décistère, est comprise exactement neuf fois dans le mètre cube. Ces mêmes dénominations appliquées à des unités de mesure différentes peuvent être la cause de tromperies ou de contestations qu'il serait facile de prévenir au moyen d'un réglement arrêté par les principaux représentants du commerce des bois. 
au gros bout, à élever ce quart au quarré, et à multiplier cette surface par la longueur de la pièce. Le volume que l'on obtient ainsi est égal aux $0,78^{\circ} \mathrm{du}$ volume cylindrique, soit un peu moins des $4 / 5^{\circ} \mathrm{du}$ volume total de la pièce.

Le cubage au cinquième déduit consiste à retrancher le cinquième de la circonférence moyenne, à prendre le quart du reste et à l'élever au quarré, puis à multiplier cette surface par la longueur de la pièce. On arrive au même résultat, en élevant au quarré le cinquième de la circonférence, et en multipliant le produit par la longueur. Le volume au cinquième déduit est sensiblement égal à la moitié $\mathrm{du}$ volume cylindrique, ou exactement 50,3 p. 0/0 du volume en grume.

Le volume au sixième déduit s'obtient en retranchant le sixième de la circonférence, en élevant au quarré le quart du reste et en multipliant le produit par la longueur de la pièce. Le volume au sixième déduit est sensiblement égal aux $0,54^{\circ}$ du volume cylindrique.

Pour abréger les calculs de cubage, on se sert de tarifs qui donnent les volumes au 1/4 sans déduction, au 1/כ̆ ou au 1/6 déduit correspondant à une circonférence et à une longueur données.

Tels sont les différents procédés usités dans le commerce pour le cubage des bois ronds. Chacun d'eux a pour objet de donner le volume utilisable dans certains cas déterminés. Ainsi le cubage au quart sans déduction s'applique souvent aux chênes 
et surtout aux sapins destinés à faire de la charpente et qui ne perdent guère qu'un cinquième de leur volume par suite d'un équarrissage ébauché. Le cubage au $1 / \mathrm{S}^{\mathrm{e}}$ déduit correspond à peu près à l'équarrissage des chênes à vive arête et sans aubier. Le cubage au $1 / 6^{\mathrm{e}}$ déduit correspond à un équarrissage un peu moins parfait.

Les résultats de ces divers procédés de cubage s'expriment toujours, comme nous l'avons dit précédemment, en mètres et décimètres cubes, ou simplement en solives ou décistères. Mais pour qu'ils soient exacts, il faut évidemment que le mesurage ait été fait rigoureusement, avec un ruban métrique, par exemple, qui ne serait susceptible ni de s'allonger, ni de se raccourcir. Or, il arrive presque toujours, dans le commerce, que ces mesurages se font avec des ficelles préparées à l'avance et dont la longueur est divisée, par des nœuds, en parties correspondant à des fractions du mètre ou du pied. Ces ficelles sont très-hygrométriques; elles s'allongent ou prennent du retrait, selon qu'elles sont mouillées ou sèches, ce qui donne lieu souvent à des fraudes assez importantes entre acheteurs et vendeurs. Ces fraudes proviennent surtout du mesurage des circonférences dont la fonction entre au carré dans l'expression du volume. Elles sont toujours très-faciles à commettre, alors même que l'on se servirait du ruban gradué, soit en mesurant la circonférence obliquement à l'axe de la pièce, soit en faisant passer le ruban sur 
des nœuds ou des renflements de la tige, soit enfin en serrant plus ou moins le ruban, ou en n'appelant pas fidèlement la graduation accusée par le mesurage.

Une autre cause de fraude encore, c'est la multiplicité et la variété des tarifs avec lesquels on calcule le volume des pièces d'après les mesures prises. Ces tarifs donnent le volume des arbres de toutes dimensions en mètres et décimètres cubes, ou en décistères ou solives. On trouve de ces comples faits dans toutes les localités forestières et, en général, ils sont appropriés par leur forme et leur construction aux usages du commerce de la contrée. Or, il est de ces tarifs qui ont été construits d'après des données trèsvariables et qui, pour une quantité de bois un peu considérable, conduisent à des résultats très-différents ; tels sont les tarifs dont la construction a pour bases les anciennes mesures en pieds et pouces, mesures variables, comme on sait, avec les localités, quoique semblables en apparence par les noms qu'elles affectent dans leurs divisions et subdivisions. Lors donc que l'on veut se servir d'un tarif pour abréger les calculs de cubage, il faut avoir soin de l'examiner avec attention, de se rendre compte exactement de la manière dont il a été construit, et d'employer, dans le mesurage de la longueur et de la circonférence des pièces, les unités linéaires et le mode de mesurage qui ont servi de bases à la construction de ce tarif. 
En général, dans le commerce, on abandonne le centimètre impair dans la mesure de la circonférence, les fractions de décimètre et le décimètre impair dans la mesure de la longueur; ou bien on mesure la circonférence de pouce en pouce en négligeant les fractions, et l'on abandonne également quelques pouces sur la longueur. - Dans la construction des tarifs, on a tenu comple de ces habitudes du commerce et, pour toutes les hauteurs, on n'y trouve que les volumes correspondant aux circonférences mesurées de deux en deux centimètres ou de pouce en pouce.

Au lieu de mesurer la circonférence moyenne des pièces à cuber, on se contente quelquefois de prendre le diamètre moyen avec un compas forestier. Dans ce cas, il faut avoir soin de présenter l'ouverture du compas dans le sens du plus grand et du plus petit diamètre, afin de déterminer le plus exactement possible le diamètre moyen. On dit qu'un arbre est méplat lorsqu'il existe une différence sensible entre le plus grand et le plus petit diamètre mesurés en un même point.

Dans le commerce des bois de charpente et dans les chantiers de la marine, le mot stère est souvent employé pour exprimer la solidité du mètre cube plein.

Le tableau ci-après est un tarif au moyen duquel on peut obtenir le volume, pour un mètre de longueur, des bois abattus : $1^{\circ}$ en grume; $2^{\circ}$ au $1 / 4$ sans déduction; $3^{\circ}$ au $1 / 6^{\mathrm{e}}$ déduit; $4^{\circ}$ au $1 / 5^{\circ}$ déduit. 
DES BOIS DE SERVICE.

\begin{tabular}{|c|c|c|c|c|c|}
\hline \multirow[b]{2}{*}{ DIAMÈTRE. } & \multirow{2}{*}{$\begin{array}{l}\text { CIRCONFÉ- } \\
\text { RENCE. }\end{array}$} & \multicolumn{4}{|c|}{ VOLJME POOR ON MĖTRE DE LONGOEUR. } \\
\hline & & $\begin{array}{c}\text { Cylindrique } \\
\text { ou en } \\
\text { grume. }\end{array}$ & $\begin{array}{c}\mathrm{Au} \mathrm{1/4} \\
\text { sans } \\
\text { déduction. }\end{array}$ & $\begin{array}{l}\text { Au 1/6 } \\
\text { déduit. }\end{array}$ & $\begin{array}{l}\text { Au 1/5̆ } \\
\text { déduit. }\end{array}$ \\
\hline $\begin{array}{l}\mathrm{m} . \\
\mathbf{0}, \mathbf{2 0}\end{array}$ & m. & m. c. & $\mathrm{m}_{0,024} \mathrm{c.}$ & $\begin{array}{c}\text { m. c. } \\
0,017\end{array}$ & $\begin{array}{c}\text { m. c. } \\
0,016\end{array}$ \\
\hline 0,22 & $\mathbf{0 , 6 9}$ & 0,038 & 0,050 & 0,021 & 0,019 \\
\hline $\mathbf{0 , 2 4}$ & 0,85 & 0,043 & 0,055 & 0,023 & 0,023 \\
\hline $\mathbf{0 , 2 5}$ & 0,28 & 0,049 & 0,038 & 0,027 & 0,025 \\
\hline $\mathbf{0 , 2 6}$ & $\mathbf{0}, \mathbf{8 2}$ & 0,053 & 0,042 & 0,029 & 0,027 \\
\hline 0,28 & 0,88 & 0,062 & 0,049 & 0,034 & 0,031 \\
\hline $\mathbf{0}, \mathbf{3 0}$ & 0,94 & 0,071 & 0.056 & 0,059 & 0,036 \\
\hline $\mathbf{0 , 3 2}$ & $\mathbf{1 , 0 0}$ & 0,080 & 0,063 & 0,044 & 0,040 \\
\hline $\mathbf{0 , 3 4}$ & $\mathbf{1}, \mathbf{0} 8$ & 0,091 & 0,071 & 0,050 & 0,046 \\
\hline $\mathbf{0 , 3 5}$ & $\mathbf{1}, \mathbf{1 0}$ & 0,096 & 0,075 & 0,0502 & 0,048 \\
\hline $\mathbf{0}, \mathbf{3 6}$ & $\mathbf{1}, \mathbf{1 3}$ & 0,102 & 0,080 & 0,0506 & 0,051 \\
\hline $\mathbf{0 , 3 8}$ & $\mathbf{1}, \mathbf{1 9}$ & 0,113 & 0,089 & 0,062 & 0,057 \\
\hline $\mathbf{0 , 4 0}$ & $\mathbf{1}, \mathbf{2 6}$ & 0,126 & 0,099 & 0,069 & 0,063 \\
\hline 0,42 & $\mathbf{1}, \mathbf{3 2}$ & 0,158 & 0,108 & 0,07כ & 0,069 \\
\hline 0,44 & 1,38 & 0,152 & 0,119 & 0,083 & 0,076 \\
\hline $0,4: 5$ & 1,41 & 0,159 & 0,125 & 0,087 & 0,080 \\
\hline $0,4 \mathbf{4}$ & 1,44 & 0,166 & 0,150 & 0,090 & 0,083 \\
\hline 0,48 & $\mathbf{1}, \mathbf{5 1}$ & 0,181 & 0,142 & 0,099 & 0,091 \\
\hline $\mathbf{0}, 50$ & 1,58 & 0,196 & 0.154 & 0,107 & 0,099 \\
\hline $\mathbf{0 , 5 2}$ & 1,63 & 0,212 & 0,166 & 0,116 & 0,107 \\
\hline $\mathbf{0 , 5 4}$ & 1,80 & 0,229 & 0,180 & 0,125 & 0,115 \\
\hline $\mathbf{0 , 5 5}$ & 1,83 & 0.238 & 0,187 & 0,150 & 0,120 \\
\hline $\mathbf{0 , 5 6}$ & 1,86 & 0,246 & 0,193 & 0,134 & 0,124 \\
\hline
\end{tabular}




\begin{tabular}{|c|c|c|c|c|c|}
\hline \multirow[b]{2}{*}{ DIAMÈTRE. } & \multirow{2}{*}{$\begin{array}{l}\text { CIRCONFÉ- } \\
\text { RENCE. }\end{array}$} & \multicolumn{4}{|c|}{ VOLUME POUR UN MĖTRE DE LONGUEUR. } \\
\hline & & $\begin{array}{c}\text { Cylindrique } \\
\text { ou en } \\
\text { grume. }\end{array}$ & $\begin{array}{c}\text { Au 1/4 } \\
\text { sans } \\
\text { déduction. }\end{array}$ & $\begin{array}{l}\text { Au } 1 / 6 \\
\text { déduit. }\end{array}$ & $\begin{array}{l}\text { Au 1/5 } \\
\text { déduit. }\end{array}$ \\
\hline $\begin{array}{l}\mathrm{m} . \\
\mathbf{0 , 5 8}\end{array}$ & m. & $\begin{array}{c}\text { m. c. } \\
0,264\end{array}$ & $\begin{array}{c}\text { m. c. } \\
0,207\end{array}$ & $\begin{array}{c}\text { m. c. } \\
0,144\end{array}$ & $\begin{array}{l}\text { m. c. } \\
0,135\end{array}$ \\
\hline $\mathbf{0 , 6 0}$ & 1,88 & $0,28 \overline{3}$ & 0,222 & 0,134 & 0,142 \\
\hline $\mathbf{0 , 6 2}$ & 1,95 & 0,302 & 0,257 & 0,163 & 0,152 \\
\hline 0,64 & $\mathbf{2}, \mathbf{0 1}$ & 0,522 & 0,253 & 0,175 & 0,162 \\
\hline $\mathbf{0 , 6 5}$ & $\mathbf{2}, \mathbf{0 4}$ & 0,552 & 0,261 & 0,181 & 0,167 \\
\hline $\mathbf{0 , 6 6}$ & $\mathbf{2}, \mathbf{0} 8$ & 0,342 & 0,268 & 0,186 & 0,172 \\
\hline 0,68 & $\mathbf{2}, \mathbf{1 3}$ & $0, \overline{5} 6 \mathbf{3}$ & 0,285 & 0,198 & 0,185 \\
\hline $\mathbf{0}, \mathbf{0}$ & $\mathbf{2}, \mathbf{2 0}$ & 0,585 & 0,302 & 0,210 & 0,194 \\
\hline $\mathbf{0}, \mathbf{2}$ & $\mathbf{2}, \mathbf{2 6}$ & 0,407 & 0,319 & 0,222 & 0,205 \\
\hline 0,21 & $\mathbf{2}, \mathbf{3} \mathbf{2}$ & 0,430 & 0,557 & 0,234 & 0,216 \\
\hline 0,25 & $\mathbf{2}, \mathbf{3 6}$ & 0,442 & 0,547 & 0,241 & 0,222 \\
\hline 0,76 & $\mathbf{2}, \mathbf{3 9}$ & 0,454 & $0, \overline{5} 56$ & 0,247 & 0,228 \\
\hline 0,8 & $\mathbf{2}, \mathbf{4} \mathbf{5}$ & 0,478 & $0, \overline{375}$ & 0,260 & $0,2 \div 0$ \\
\hline 0,80 & 2,51 & $0, \check{03}$ & $0, \overline{395}$ & 0,274 & 0,255 \\
\hline 0,82 & $2, \overline{5}$ & $0, こ 28$ & 0,414 & 0,288 & 0,266 \\
\hline $\mathbf{0}, \mathbf{8}^{4}$ & $\mathbf{2 , 6 4}$ & 0,354 & $0,4 \overline{3} 5$ & 0,502 & 0,279 \\
\hline 0,85 & $2,6 z$ & 0,567 & 0,445 & 0,509 & 0,285 \\
\hline $\mathbf{0}, \AA \mathbf{6}$ & 2,70 & 0,581 & 0,456 & 0,317 & 0,292 \\
\hline 0,88 & 2,86 & 0,608 & 0,477 & 0,531 & $0, \overline{3} 06$ \\
\hline $\mathbf{0}, \mathbf{9 0}$ & $\mathbf{2}, \mathbf{8 3}$ & 0,636 & 0,499 & 0,347 & $0, \overline{3} 20$ \\
\hline $\mathbf{0 , 9 2}$ & $\mathbf{2 , 8 9}$ & 0,665 & 0,522 & 0,362 & 0,354 \\
\hline 0,94 & $\mathbf{2 , 9 5}$ & 0,694 & 0,545 & $0, \overline{578}$ & $0, \overline{3} 49$ \\
\hline
\end{tabular}


DES BOIS DE SERVICE.

\begin{tabular}{|c|c|c|c|c|c|}
\hline \multirow[b]{2}{*}{ DIA NÈTRE. } & \multirow{2}{*}{$\begin{array}{l}\text { CIRCONFÉ- } \\
\text { RENCE. }\end{array}$} & \multicolumn{4}{|c|}{ VOLUIIE POUR UN HETRE DE LONGJEUR. } \\
\hline & & $\begin{array}{c}\text { Cylindrique } \\
\text { ou en } \\
\text { grume. }\end{array}$ & $\begin{array}{c}\text { Au 1/4 } \\
\text { sans } \\
\text { déduction. }\end{array}$ & $\begin{array}{l}\text { Au 1,6 } \\
\text { déduit. }\end{array}$ & $\begin{array}{l}\text { Au 1/J } \\
\text { déduit. }\end{array}$ \\
\hline $\begin{array}{l}\mathrm{m} . \\
\mathbf{0}, \mathbf{9}\end{array}$ & $\stackrel{\text { m. }}{2,98}$ & $\begin{array}{r}\text { m. } \mathbf{c .} \\
0,709\end{array}$ & $\begin{array}{c}\text { m. c. } \\
0, \text { כ̆ฐ6 }\end{array}$ & $\begin{array}{l}\text { m. c. } \\
0,586\end{array}$ & $\begin{array}{l}\text { m. } \\
0, \bar{z} 57\end{array}$ \\
\hline $\mathbf{0 , 9 6}$ & $\mathbf{3}, \mathbf{0} 1$ & 0,724 & 0), こ68 & $0,59 \check{3}$ & 0,364 \\
\hline (1,98 & $\mathbf{3}, \mathbf{0 8}$ & 0,754 & $0, \breve{591}$ & 0,411 & 0,379 \\
\hline $\mathbf{1}, \mathbf{0 0}$ & 3, 14 & 0,783 & 0,616 & 0,428 & $0, \overline{5} 9 \check{~}$ \\
\hline $\mathbf{1}, \mathbf{0} 2$ & $\mathbf{3}, \mathbf{2 0}$ & 0,817 & 0,641 & 0,445 & 0,411 \\
\hline 1,04 & $\mathbf{3}, \mathbf{2} 2$ & 0,849 & 0,666 & 0,463 & 0,427 \\
\hline 1,05 & $\mathbf{3}, \mathbf{3}$ & 0,866 & 0,680 & 0,472 & 0,450 \\
\hline $\mathbf{1}, \mathbf{0 6}$ & $\mathbf{3}, \mathbf{3 3}$ & 0,882 & 0,692 & 0,481 & 0,444 \\
\hline $\mathbf{1}, 08$ & $\mathbf{3}, \mathbf{3 9}$ & 0,916 & 0,719 & 0,499 & 0,461 \\
\hline 1,10 & $\mathbf{3}, \mathbf{4 5}$ & 0,950 & 0,746 & 0,518 & 0,478 \\
\hline 1,12 & $\mathbf{3}, \mathbf{5 9}$ & $0,983 ้$ & 0,773 & 0,5557 & 0,495 \\
\hline 1,14 & $\mathbf{3 , 5 8}$ & 1,021 & 0,801 & $0,5 ็ 56$ & 0,514 \\
\hline 1,15 & $\mathbf{3}, \mathbf{6 1}$ & 1,039 & 0,816 & 0,566 & 0,325 \\
\hline 1,16 & 3,61 & 1,057 & 0,850 & 0,576 & 0,552 \\
\hline 1,18 & 3,80 & 1,094 & $0,85 \% 8$ & $0, \check{9} 96$ & 0, วั50 \\
\hline $\mathbf{1}, \mathbf{2 0}$ & 3,86 & 1,131 & 0,888 & 0,616 & $0, \check{569}$ \\
\hline $\mathbf{1}, \mathbf{2} 2$ & $3 \cdot 83$ & 1,169 & 0,918 & 0,657 & $0, \preceq 88$ \\
\hline $1,24$. & 3,89 & 1,208 & 0,948 & 0,658 & 0,608 \\
\hline 1,25 & $\mathbf{3}, \mathbf{9} \mathbf{3}$ & 1,227 & 0,963 & 0,669 & 0,617 \\
\hline 1,26 & $\mathbf{3 , 9 6}$ & 1,247 & 0,979 & 0,680 & 0,627 \\
\hline 1,28 & 4,02 & 1,287 & $1,0\rfloor 0$ & 0,701 & 0,647 \\
\hline 1,30 & 4,08 & $1, \overline{327}$ & 1,042 & 0,725 & 0,667 \\
\hline
\end{tabular}


S III. - Mode de mesurage, de cubage et de vente des bois équarris ou carrés.

Les différents procédés de cubage que nous venons d'indiquer s'appliquent particulièrement aux bois ronds ou en grume, avec ou sans écorce, tels qu'ils ont été découpés, après l'abatage, dans les coupes en exploitation. Les bois de travail ou d'industrie se vendent et se cubent ordinairement sous cette forme. Quant aux bois de service qui doivent être exportés loin du lieu de production, nous avons dit qu'on les équarrit sur le parterre des coupes, afin de rendre leur transport plus facile et moins coûteux. Tels sont, pour la plupart, les bois de charpente de chêne qui alimentent'le commerce de Paris; tels sont aussi les bois de chêne que l'on destine aux constructions navales. Les bois de commerce sont souvent peu équarris, tandis que les bois façonnés sur devis sont quelquefois équarris à vive arête, c'est-à-dire, de manière que les angles soient bien marqués, de bois dur et solide, et sans aubier.

Par le mot équarrissage, on entend aussi, en terme de cubage, les dimensions en largeur et en épaisseur d'une pièce de bois équarrie. - On dit qu'une pièce a 16 centimètres ( 6 pouces) d'équarrissage, si elle a cette mesure sur chaque face. $0 \mathrm{n}$ dit qu'une pièce a 16 sur 22 centimètres ( 6 sur 8 pouces) d'équarrissage, quand elle a 16 centimètres d'épaisseur sur 22 centimètres de largeur. 
Pour cuber une pièce de bois équarrie, on la considère comme un parallélipipède rectangle ayant pour hauteur la longueur de la pièce, et pour base le rectangle formé par les mesures d'équarrissage prises au milieu ou par la moyenne des mesures d'équarrissage prises au petit et au gros bout. Les côtés d'équarrissage se mesurent avec une équerre qui se compose d'une règle divisée en centimètres ou en pouces, et portant à l'une de ses extrémités une autre règle fixée à angle droit.

Les bois de commerce étant souvent équarris fort irrégulièrement, des règlements particuliers déterminent le mode de mesurage et de cubage de ces bois dans chaque localité. Ainsi, à Paris, le mesurage des dimensions de la base se fait de 3 en 3 centimètres pleins, et celui des longueurs de $2 \breve{5}$ en $2 \breve{\partial}$ centimètres pleins (arrêté des entrepreneurs de char-

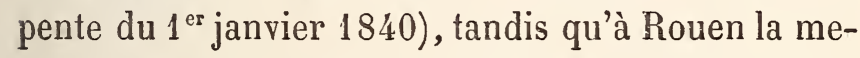
sure de l'équarrissage se prend de 2 en 2 centimètres, et les longueurs de 20 en 20 centimètres. Le volume de ces bois se calcule ensuite, de même que celui des bois ronds, à l'aide d'un tarif construit sur les bases adoptées dans la localité pour le mesurage de la longueur des pièces et des côtés d'équarrissage.

Le tableau suivant permet de calculer avec rapidité le volume des bois équarris dans les conditions où on les emploie habituellement dans les constructions. 
POUR LES BOIS ÉQUARRIS ET POUR UN MÈTRE DE LONGUEUR.

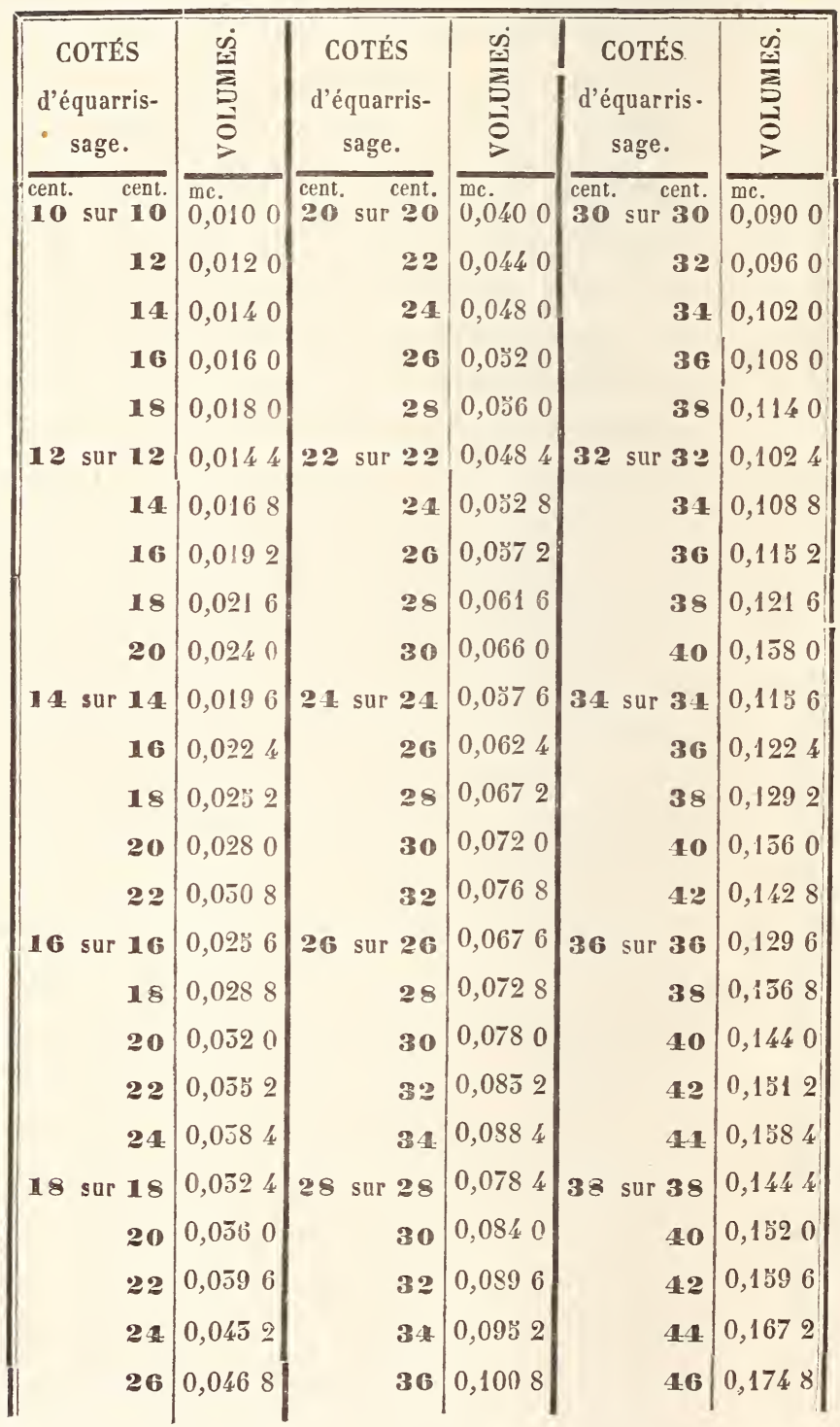




\begin{tabular}{|c|c|c|c|c|c|c|}
\hline $\begin{array}{c}\text { COTES } \\
\text { d'équarris- } \\
\text { sage. }\end{array}$ & \multirow{2}{*}{ 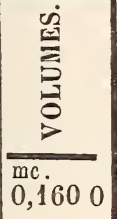 } & \multicolumn{2}{|c|}{$\begin{array}{l}\text { COTÉS } \\
\text { d'équarris- } \\
\text { sage. }\end{array}$} & \multirow{2}{*}{ 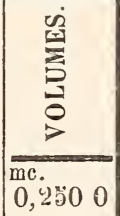 } & $\begin{array}{c}\text { COTÉS } \\
\text { d'équarris- } \\
\text { sage. }\end{array}$ & \multirow{2}{*}{ 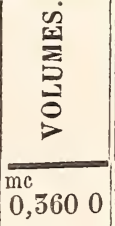 } \\
\hline $\begin{array}{l}\text { cent. cent. } \\
\mathbf{1 0} \text { sur } \mathbf{1 0}\end{array}$ & & cent. sur & $\begin{array}{l}\text { cent. } \\
\mathbf{5 0}\end{array}$ & & ent. cent & \\
\hline 42 & 0,1680 & & 52 & 0,2600 & 62 & 0,3720 \\
\hline 4.1 & 0,1760 & & 54 & 0,2700 & 64. & 0,5840 \\
\hline 46 & 0,1840 & & 56 & 0,2800 & 66 & 0,5960 \\
\hline 48 & 0,1920 & & 58 & 0,2900 & 68 & 0,4080 \\
\hline $\mathbf{4 2}$ sur $\mathbf{1 2}$ & 0,1764 & 52 sur & 52 & 0,2704 & $62 \operatorname{sur} 62$ & 0,3844 \\
\hline 44 & 0,1848 & & 54 & 0,2808 & 64 & 0,3968 \\
\hline 46 & 0,1932 & & 56 & 0,2912 & 66 & 0,4092 \\
\hline 48 & $0 ; 2016$ & & 58 & 0,3016 & 68 & 0 \\
\hline 50 & 0,2100 & & 60 & 0, & 80 & 0,4340 \\
\hline 4.1. $\operatorname{sur} 41$ & 0,1956 & 54 sur & 54 & 16 & 64 sur 64 & 0,4096 \\
\hline 46 & 0,2024 & & 56 & $0, \overline{5} 024$ & 66 & 0,4224 \\
\hline 48 & 0,2112 & & 58 & 0,5152 & 68 & 0, \\
\hline 50 & 0,2 & & 60 & 0,5240 & 80 & 0 , \\
\hline 52 & 0,2 & & 62 & 0 & 82 & 0 \\
\hline 46 sur $\mathbf{4 6}$ & 0,2 & 56 sur & 56 & & 66 sur 66 & 6 \\
\hline 48 & 0,2208 & & 58 & 0 & 68 & 0,4488 \\
\hline 50 & 0,2500 & & 60 & 0 & 80 & 0,4 \\
\hline 52 & 0,2592 & & 62 & & 22 & 0,4752 \\
\hline 54 & 0,2484 & & 64 & 84 & 84 & 0 \\
\hline 48 sur $\mathbf{4 8}$ & 0,2 & 58 sur & 58 & 0,5564 & 68 sur 68 & 0, \\
\hline $\mathbf{5 0}$ & 0, & & 60 & 0 & 80 & 0,4760 \\
\hline 52 & 0,2496 & & 62 & 0,5596 & 82 & 0 \\
\hline 54 & 0,2092 & & 64 & 12 & 84 & 0 \\
\hline 56 & 0,2688 & & 66 & 0,5828 & $\begin{array}{r}86 \\
80 \\
8 u r 80\end{array}$ & $\begin{array}{l}0,516 \\
0,490\end{array}$ \\
\hline
\end{tabular}


Article III.

Mode de débit, de mesurage et de vente des bois de travail ou d'industrie faconnés dans les coupes.

\section{§ I. - Sciages de chêne.}

Les bois de travail que l'on façonne dans les coupes sont livrés aux ouvriers des différents métiers, pour la plus grande partie, en planches de diverses épaisseurs. Le chêne, le hêtre, le sapin, l'épicéa, le pin sylvestre et les peupliers sont les essences qui fournissent le plus de planches à la consommation. On donne le nom de madriers aux planches qui ont plus de $0^{\mathrm{m}}, 08$ d'épaisseur.

Les sciages de chêne se font ordinairement sur le parterre même des coupes en exploitation. On emploie pour ce genre de débit des ouvriers spéciaux que l'on nomme scieurs de long (1). Tout le monde sait comment est organisé l'atelier des scieurs de long; nous n'en parlerons pas. Avant de monter la pièce qui doit être sciée, soit sur tréteaux, soit sur chevalet (on se sert le plus ordinairement de chevalets dans les coupes), les ouvriers équarrissent légèrement le bois sur 4,6 ou 8 faces, puis ils tracent sur la pièce les traits qu'ils doivent suivre en la dé-

(1) Depuis quelques années, on a monté de petites scieries à vapeur que l'on transporte successivement dans les coupes où il y a du bois à débiter. L'expérience n'a pas encore démontré s'il y a intérêt à substituer ces machines aux ateliers de scieurs de long. 
DES BOIS DE TRAVALL OU D'INDUSTRIE.

bitant. Ces traits se marquent avec un cordeau frotté dans du charbon délayé dans de l'eau. C'est l'ouvrier monté sur le chevalet qui dirige la scie suivant le trait; par conséquent, dans chaque atelier de scieurs, c'est le plus habile qui doit occuper ce poste.

Le sciage peut être dirigé simplement suivant des plans parallèles entre eux dans toute l'épaisseur de la pièce. Ce genre de débit tout primitif est, pour le chêne, fort inférieur aux autres; on l'emploie spécialement pour faire des planches dites de bateau, qui servent à construire des barques et des bateaux pour la navigation des rivières et des canaux, ou pour la pêche et le cabotage sur le littoral de la mer.

Le plus souvent on opère de la manière suivante. Après un équarrissage grossier, à la hache, des quatre faces $a a$. ., (voir la figure 2) on enlève avec la scie deux planches irrégulières par leurs bords, $b$ et $c$, appelées déballes, d'une épaisseur assez forte pour qu'elles emportent une bonne partie d'aubier sur leurs bords en laissant à la pièce deux belles faces planes; ensuite on détache un talon $d$, d'une épaisseur variable et qui sera débité à part; puis enfin l'on débite la pièce ainsi préparée et formant un ou plusieurs bancs en planches ou madriers de diverses dimensions et combinés de manière à donner les sciages les plus avantageux. Le tracé dı sciage exige alors beaucoup d'habitude et même d'habileté de la part de l'ouvrier qui en est chargé, sans quoi l'on ne tire pas le meilleur parti possible de la pièce à débiter; 
et, comme les scieurs de long travaillent ordinairement à la tâche, le propriétaire du bois à scier doit surveiller de près celte opération, pour empêcher les ouvriers de diriger le sciage uniquement dans leur intérêt, qui souvent diffère du sien.

Le tracé du sciage influe aussi sur la qualité et la beauté de la planche; on le voit aisément par les planches que l'on obtient dans le sciage sur maille. Ce genre de débit consiste à mener le trait de scie de la circonférence au cœur de la pièce, c'est-à-dire suivant la direction des rayons médullaires. On conçoit donc, a priori, qu'une pièce de bois ne peut pas être sciée tout entière de celte manière, mais on obtient la plus grande surface possible de planches sur maille en suivant le tracé indiqué par la figure 3. Les planches sur maille du chêne et de toutes les essences à gros rayons se reconnaissent facilement par les taches d'un blanc nacré et de formes diverses dont elles sont émaillées. Non-seulement elles sont plus belles et, par conséquent, plus recherchées pour la menuiserie et l'ébénisterie que les planches débitées dans une direction perpendiculaire aux rayons, mais elles font un usage beaucoup meilleur, elles s'usent moins vite dans certains emplois comme les planchers et les parquets, et elles se gercent et se tourmentent moins dans les ouvrages de menuiserie comme les panneaux de portes, les lambris, etc.

On évite cependant, dans le débit de certaines pièces, de diriger exactement le trait de scie dans le 
sens des rayons médullaires, afin que la planche ou le madrier ne renferme aucune partie du cour de l'arbre. Cette précaution est souvent exigée dans le sciage des bois que l'on débite sur devis, notanment dans le sciage des planches ou madriers destinés à la construction des wagons (1), parce que le bois du cœur est quelquefois de moins bonne qualité, et qu'il éclate, se tourmente et se détache plus facilement, lorsque la pièce est mise en ouvre.

Le chêne rouvre, que les ouvriers de certaines localités appellent le drille ou le drillard, est préférable, en général, au pédonculé pour la belle menuiserie, l'ébénisterie et la sculpture; son bois est plus doux, plus facile à travailler, et il se gerce et se tourmente moins, quand il est mis en œurre, que celui du chêne pédonculé.

Hors le cas où on travaille sur devis, et où l'on tient exclusivement à scier sur maille, les propriétaires ou les marchands font débiter leurs bois suivant des dimensions déterminées et conformes aux

(1) En France, les compagnies de chemins de fer possédaient ensemble, au $1^{\text {er }}$ janvier $1866,90.000$ wagons à marchandises el environ 15.000 voitures à voyageurs. La quantité de bois employée dans la construction de chaque wagon ou voiture varie de 3 à 8 mètres cubes (cube net), comprenant toujours du chêne pour $\mathbf{3}$ mètres cubes à peu près, puis du sapin, du frène, de l'orme et autres bois pour le surplus. Il en résulte que la construction seule de ce matériel a absorbé en bois de chêne de premier choix 300.000 mètres cubes employés, soit au moins le double en grume. La durée de ce matériel roulant n'est pas encore bien connue; mais ou n'estime pas à moins de $\mathbf{3 0 . 0 0 0}$ mètres cubes en grume la quantité de bois de chène nécessaire à son entretien. 
usages du commerce local. Dans le centre de la France on débite les chênes en planches et madriers dont les dimensions varient avec celles des arbres à scier. Mais la planche murchande ou ordinaire, dans laquelle on laisse subsister l'aubier, doit avoir au minimum $0^{\mathrm{m}}, 27$ à $0^{\mathrm{m}}, 28$ ( 10 pouces) de largeur sur $0^{\mathrm{m}}, 03$ (1 pouce) d'épaisseur. Cette planche se vend au mètre carré. - Il en est de même des madriers dont le prix se règle de la même manière, et augmente proportionnellement à l'épaisseur. - Le déchet dû à ce mode de sciage n'est que de 22 à 2 ว p. $\% / 0 \mathrm{du}$ volume en grume.

Ailleurs, et surtout dans les localités dont les produits alimentent le commerce de Paris, on a adopté les noms et les dimensions des planches du débit de Paris, savoir :

Le grand battant, largenr, $0,{ }^{\mathrm{m}} \overline{5} 5 \bar{j}$, épaisseur, $0, \mathrm{~m}_{11}$, ou $12 / 4$ pouces.

\begin{tabular}{|c|c|c|c|c|c|}
\hline Le petit battant, & - & 0,23 & - & 0,08 , ou $9 / 5$ & 一 \\
\hline La doublette, & - & $0,3 \overline{3} \overline{,}$ & - & 0,06, ou $12 / 2$ & \\
\hline L'échantillon, & & 0,25 , & - & 0,04 , ou 9 p. sur & $11 /$ \\
\hline La membrure, & & 0,165 & - & 0,08 , ou $6 / \overline{3}$ po & puces. \\
\hline revous, & & 0,25 & - & 0,03 , ou $9 / 1$ & - \\
\hline evron, & & 0,08 , & 一 & 0,08 , ou $3 / 3$ & - \\
\hline zembrette, & & $0, \quad 18$, & $0^{\mathrm{m}}$ & à 0,06 , ou $6 / 2$ & 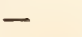 \\
\hline
\end{tabular}

La frise, ou planche à parquet, largeur $0,{ }^{\mathrm{m}} 12$ à $0,{ }^{\mathrm{m}} 13$, épaisseur, $0,{ }^{\mathrm{m}} 03$, ou 4 pouces de largeur sur 1 pouce d'épaisseur.

On fait encore avec le chêne des planches moins épaisses dont les plus en usage sont : 
DES BOIS DE TRAVAIL OU D'INDUSTRIE.

Le panneau, largeur, $0^{\mathrm{m}}, 22$ à $0^{\mathrm{m}}, 24$, épaisseur, $0^{\mathrm{m}}, 020$ à $0^{\mathrm{m}}, 022$ on 9 pouces sur 9 lignes.

La volige, largeur, $0^{\mathrm{m}}, 22$ à $0^{\mathrm{m}}, 24$, épaisseur, $0^{\mathrm{m}}, 013$ à $0^{\mathrm{m}}, 015$ ou

9 pouces sur 6 lignes.

Le feuillet, largeur, $0^{\mathrm{m}}, 22$ à $0^{\mathrm{m}}, 24$, épaisseur, $0 \mathrm{~m}, 006$ à $0^{\mathrm{m}}, 007$ ou

9 pouces sur 5 lignes.

Ces trois planches, les deux dernières surtout, étant spécialement employées dans la menuiserie de luxe et dans l'ébénisterie, doivent être débitées autant que possible sur maille, et ne peuvent être fournies que par des chênes de choix, à grain fin, et sans nœuds.

Quant à la longueur des planches, elle varie sans cependant descendre au-dessous de 2 mètres (1), excepté pour les frises que l'on débite souvent à la longueur de 1 mètre ou 1 mètre 50.

Les planches du débit de Paris ne doivent point contenir d'aubier, et se vendent ordinairement par lots assortis de différentes pièces. Dans ce cas, l'unité de vente est le cent de toises courantes, ou les deux cents mètres mesurés dans la longueur. Dans le toisé de ces planches, on mesure les longueurs de $0^{\mathrm{m}}, 25$ en $0^{\mathrm{m}}, 25$, de sorte qu'une planche qui porte $2^{\mathrm{m}}, 20$ de longueur ne compte que pour 2 mètres; et comme les planches nint des dimensions très-différentes, sous le rapport de la largeur el de l'épaisseur,

(1) On fait quelquefois des planches qui n'ont que 1 mètre ou 1 mètre 50 de longueur, mais elles sont moins estimées et se payent relativement moins cher que les planches plus longues, parce qu'en général elles proviennent des branches, ou de chênes éboutés par suite de vices. 
on les compare et on les rapporte toutes à deux types, l'échantillon et l'entrevous, dont les volumes sont des sous-multiples ou des multiples du volume des autres. C'est ainsi que :

Le grand battant vaut quatre échantillons ou six entrevous ;

Le petit battant vaut deux échantillons ou trois entrevous;

La doubletle vaut deux échantillons ou trois entrevous ;

La membrure vaut un échantillon fort;

Le chevron vaut un entrevous ;

La membrette vaut un entrevous faible ou un demi-échantillon fort ;

La frise vaut un demi-entrevous.

On peut donc exprimer la valeur de toutes les pièces d'un lot de sciage en échantillons ou en entrevous; et comme l'entrevous vaut lui-même les $3 / 4$ de l'échantillon, il en résulte que quand l'échantillon vaut $200 \mathrm{fr}$. (le cent de toises, ou les 200 mètres), l'entrevous vaut 1 วั0 à $160 \mathrm{fr}$. Les prix de ces deux espèces de marchandises se rapprochent ou s'écartent souvent un peu plus, selon les besoins ou la demande du commerce.

I.es sciages de chêne se vendent le plus souvent par lots spéciaux d'échantillons comprenant : échantillon, membrure, doublette, petit et grand battant; ou par lots d'entrevous formés d'entrevous, de chevron et de membrette. D'après les usages du commerce, les lots d'échantillons doivent toujours 
comprendre 10 à $15 \mathrm{p}$. $0 / 0$ de membrures et même proportion de doublettes. La membrure seule, quand elle est bien conditionnée, se paye toujours mieux que l'échantillon.

La frise, ou planche à parquet, qui représente, pour la largeur, un entrevous scié en deux, se vend ordinairement à part et moins cher, proportionnellement, que l'entrevous de même qualité.

Dans la vente des planches, il est d'usage assez général d'accorder à l'acheteur 10 p. 0/0 de fourniture, c'est-à-dire que le vendeur lui livre 110 mètres au lieu de cent, ou 220 mètres pour le grand cent.

En moyenne, on calcule qu'un mètre cube de bois en grume donne 50 mètres courants d'échantillon; d'où il suit qu'il faut 4 mètres cubes en grume, ou 2 mètres cubes au $5^{\circ}$ déduit, pour 100 toises d'échantillon, ou encore une solive de bois au $\breve{5}^{\mathrm{e}}$ pour donner 5 toises ou 10 mètres d'échantillon.

On calcule de même qu'il faut $2^{\text {m.c. }}, 600$ à $3^{\text {m.c. }}, 000$ de bois rond, ou $1^{\text {m.c. }}, 300$ à $1^{\text {m.c. }}, 500$ au $5^{\mathrm{e}}$ déduit, pour fournir le cent de toises d'entrevous (1).

(1) Ces chiffres sont des moyennes qui varient avec les dimensions et la forme des pièces. Plus les arbres sont gros, toutes choses égales d'ailleurs, moins il y a, relativement au volume brut, de déchet dans le débit en marchandises. Le déchet est de moitié environ, quand le travail est fait dans les coupes par des scieurs de long, et il est sensiblement moindre dans les scieries mécaniques où l'on fait usage de scies à lames plus minces, et où l'outillage permel d'utiliser tout le bois parfait. 


\section{§II. - Sciages de hêtre.}

Le hêtre que l'on scie en planches et madriers est surtout employé par les menuisiers et les ébénistes à la fabrication des meubles. Dans beaucoup de localités, ce genre de débit du hêtre n'est soumis à aucune règle fixe; mais dans les forêts dont les produits sont destinés à l'alimentation de grands centres de fabrication, comme Paris, on tend généralement à adopter un mode de débit uniforme, lequel se rapproche beaucoup de celui du chêne. C'est ainsi qu'à Villers-Cotterets, l'une des contrées qui fournissent le plus de sciage de hêtre à la capitale, un règlement convenu entre les exploitants et la Compagnie des marchands de bois de Paris, règlement approuvé par une décision ministérielle du mois de mai 1835, détermine les dimensions à donner aux planches de hêtre, les réductions à faire subir à celles qui n'auraient pas les dimensions prescrites, et enfin les causes qui peuvent faire rebuter ou refuser par l'acheteur les bois qui lui auraient été expédiés par le vendeur. D'après ce règlement, les sciages de hêtre comprennent plus spécialement quatre échantillons que l'on désigne sous les noms de : entrevous ou feuillet, membrure, doublette ou trappe, et quartelot.

L'entrevous, ou feuillet de hêtre, est la planche que l'on fabrique le plus abondamment. Ses dimensions en largeur et en épaisseur sont généralement de $0^{\mathrm{m}}, 216$ à $0^{\mathrm{m}}, 243$, sur $0^{\mathrm{m}}, 033$ à $0^{\mathrm{m}}, 031$, et doivent 
DES bOIS DE TRAVAll OU D'industrie.

toujours être telles qu'une section droite en travers de la pièce donne une surface de $0^{\text {m.q. }}, 0073$. C'est, comme on voit, une pièce de même volume, ou à peu près, que l'entrevous ordinaire de chêne.

La membrure de hêtre n'a pas de dimensions fixes, mais la section droite doit présenter une surface de $0^{\text {m.q. }}, 0154$ à $0^{\text {m.q. }}, 0175$. On lui donne ordinairement $0^{\mathrm{m}}, 165$ de largeur sur $0^{\mathrm{m}}, 110$ d'épaisseur, ou $0^{\mathrm{m}}, 180$ sur $0^{\mathrm{m}}, 100$, ou $0^{\mathrm{m}}, 200$ sur 0,080 . La membrure de hêtre est, par conséquent, plus forte que celle de chêne, à laquelle on ne donne que $0^{\mathrm{m}}, 165$ sur $0^{\mathrm{m}}, 08$.

La doublette ou trappe de hêtre porte généralement $0^{\mathrm{m}}, 33$ de largeur sur $0^{\mathrm{m}}, 075$ à $0^{\mathrm{m}}, 081$ d'épaisseur, et doit présenter une surface de $0^{\text {m.q. }}, 0254$ à $0^{\mathrm{m} . q .}, 0277$ sur une section droite en travers de la pièce. Elle est plus forte que la doublette de chêne dont la largeur est la même et dont l'épaisseur ne dépasse pas $0^{\mathrm{m}}, 062$.

Le quartelot de hêtre est une planche qui porte

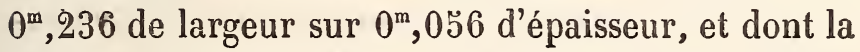
section doit avoir une surface de $0^{\text {m.q. }}, 0123$ à $0^{\text {m.q. }}, 0139$. Par son volume, cette pièce peut se comparer à un fort échantillon ou, plus exactement, à une membrure de chêne.

En résumé, dans ce débit du hêtre, l'entrevous représente la planche mince et le quartelot la planche épaisse; mais dans chacun des types, c'est la surface de la section transversale qui est fixée, bien plus que chacune de ses dimensions. 
Les planches de hêtre n'ont pas de longueur déterminée; elles se vendent à Paris, comme celles de chêne, au cent de toises, ou aux deux cents mètres courants, ou au grand cent, ce qui est la même chose. De même aussi, dans le toisé de ces planches, on les rapporte toutes à un seul type qui est la membrure ou le quartelot; la doublette compte pour deux, l'entrevous pour deux tiers.

On voit, par cet exemple, que le débit régulier du hêtre se rapproche de celui du chêne, et ne diffère de celui-ci qu'en ce que les planches de hêtre ont plus d'épaisseur que les planches correspondantes de chêne.

Le règlement de Villers-Cotterets fixe également les dimensions à donner aux planches de chêne. Ces dimensions en largeur et en épaisseur ne sont pas exactement celles adoptées pour le débit régulier de Paris, mais, pour une même longueur, le volume du feuillet, de la membrure, de la doublette et du quartelot de chêne, est le même que celui de l'entrevous, de la membrure, de la doublette et de l'échantillon du débit de Paris.

Avec le hêtre on fabrique aussi des madriers épais, appelés étaux, qui servent à faire des tables de boucherie ou de cuisine, des établis de menuisier, etc., et des planches minces, dites de petit sciage, qui sont employées à une infinité d'usages dans les arts. Ces menues planches ont une longueur uniforme de $2^{\mathrm{m}}, 25$ sur $0^{\mathrm{m}}, 11$ à $0^{\mathrm{m}}, 25$ de largeur et $0^{\mathrm{m}}, 015$ à $0^{\mathrm{m}}, 006$ d'épaisseur, et se vendent par bottes. 
Les sciages de hêtre n'ont qu'une importance secondaire et le prix des bois qui se débitent de cette façon n'est pas beaucoup plus élevé que celui du hêtre destiné au chauffage ; il ne dépasse guère dans l'Est 16 francs le mètre cube en grume et sur pied.

\section{\$II. - Sciages de sapin.}

Le sapin, l'épicéa et le pin sylvestre fournissent des planches dont on fait une grande consommation, surtout en menuiserie, et que l'on désigne indistinctement dans le commerce sous le nom de planches ou sciages de sapin.

Le mode de débit du sapin en planches diffère suivant les localités. Dans certaines contrées, on donne à la planche des longueurs et des largeurs variables avec les dimensions des pièces à scier, et une épaisseur uniforme de $0^{\mathrm{m}}, 027$ ou un pouce. Ce genre de sciage, qui est encore pratiqué dans quelques localités de l'Alsace et de la Franche-Comté, s'appelait autrefois débít de Strasbourg; il consiste à diriger tous les traits de scie dans le même sens, de telle sorte, qu'après le sciage, les planches peuvent se replacer les unes sur les autres et recomposer la pièce. Mais aujourd'hui on tend à adopter, dans les sapinières les plus importantes de France, le mode de débit usité depuis des siècles dans les Vosges, et appelé pour cette raison débit des Vosges.

Tandis que dans le Jura et les Alpes françaises on 
ne débite en planches qu'une faible partie du produit des sapinières, dans les Vosges, au contraire, la grande majorité des sapins sont sciés sur place, suivant les règles que nous allons faire connaître.

Les arbres destinés au sciage sont découpés sur place en troncons, que l'on nomme tronces de sciage, et auxquels on donne ordinairement $3^{\mathrm{m}}, \mathrm{s} 7$ à $3^{\mathrm{m}}, 90$ (11 ou 12 pieds) de longueur (1). La découpe de ces bois en forêt facilite la vidange et le transport des tronces dans les scieries où elles doivent être débitées.

Les planches que l'on fabrique dans les scieries des Vosges sont de dimensions diverses, mais elles peuvent se comparer ou se réduire toutes à un même type qui a :

de longueur $3^{\mathrm{m}}, 90$, ou 12 pieds, de largeur $0^{\mathrm{m}}, 244$, ou 9 pouces, d'épaisseur $0^{\mathrm{m}}, 027$, ou 1 pouce.

Cette planche se désigne ordinairement sous lo nom de planche de 12/9 (12 pieds de longueur, sur 9 pouces de largeur et 1 pouce d'épaisseur).

Les autres planches du débit des Vosges ont pour dimensions : 12/12,11/12,11/9,12/8,11/8. Toutes ces planches sont supposées avoir un pouce $\left(0^{\mathrm{m}}, 027\right)$ d'épaisseur, mais en général elles sont moins épaisses, surtout quand elles sont sèches. Elles n'ont le plus

(1) Quelques marchands donnent aux tronces de scia gre les dimensions métriques de $3^{\mathrm{m}}, 66$ à $4 \mathrm{~m}$, mais le plus grand nombre font mesurer et débiter leurs tronces à l'ancien pied-de-roi $\left(0^{\mathrm{m}}, 525\right)$. 
souvent que 11 lignes à l'extrémité correspondant au gros bout de la tronce, et 10 lignes au bout opposé. Quelquefois cependant, on donne 15 lignes d'épaisseur à la planche de 12/12 et 2 pouces ou 54 millimètres à la planche de 12/9, mais ces sortes de planches ne se fabriquent que sur commande.

Toutes ces planches reçoivent dans le commerce des dénominations différentes. On appelle :

Planche ordinaire, ou marchande, celle de 12/9 et quelquefois de $11 / 9$;

Planche réduite, celle de 12/8 et quelquefois de $11 / 8$

Planche large, celle de 12/12 et quelquefois de $11,12$.

Dans le sciage, la première planche détachée de chaque tronce se nomme dosseau; l'une de ses faces est plane, tandis que l'autre conserve la forme extérieure et convexe de l'arbre.

On appelle chons, les planches que l'on retire immédiatement après les dosseaux, et dont les côtés sont encore flacheux ou en biseau. Les chons ont une largeur moyenne de 6 à 7 pouces $\left(0^{\mathrm{m}}, 162\right.$ à $\left.0^{\mathrm{m}}, 189\right)$.

On appelle rebut ou planche de rebut, une planche fendue, trouée, cranée, ou qui renferme des nœuds susceptibles de se détacher et de former des trous après la dessiccation du bois.

Avec les rebuts et les chons, on fabrique souvent des lattes que l'on refend à la scie. Les lattes n'ont pas de largeur fixe ; on estime qu'il faut quatre lattes pour une planche de 8 pouces. 
On estime, en moyenne, qu'un mètre cube de sapin en grume, avec écorce, peut donner 26 à 27 planches ordinaires et chons. Le déchet est alors d'environ $34 \mathrm{p} .0 / 0$ ou du tiers du volume en grume. Mais le nombre des planches varie naturellement avec les dimensions des arbres à débiter; il est plus fort ou plus faible, selon la grosseur des tronces de sciage, ef peut se déduire très-approximativement du diamètre au petit bout de la pièce à scier.

En effet, le volume de la tronce en grume est donné par la formule :

$$
1 / 4 \pi \mathrm{D}^{2} \mathrm{H} \text {. }
$$

Le volume de la planche ordinaire de 9 pouces est égal à $9 \times 1 \times \mathbf{H}$; d'où le nombre de planches ordinaires contenu dans la tronce

$$
=\frac{1 / 4 \pi \mathrm{D}^{4} \mathrm{H}}{9 \times 1 \times \mathrm{H}}=\frac{\pi \mathrm{D}^{2}}{4 \times 9}=\frac{\mathrm{D}^{2}}{12}
$$

(en faisant $\pi=3$ pour tenir compte du trait de scie).

Ce qui veut dire que le rendement en planches de 12/9, d'une bille de sapin de 12 pieds de longueur, est égal au carré du petit diamètre (mesuré en pouces) divisé par 12.

Ainsi, une tronce de 24 pouces de diamètre au petit bout donne 48 planches, dont assez ordinairement :

18 planches, $1^{\text {re }}$ qualité, sans nœuds, soit $3 / 8$;

$24-2^{\mathrm{e}}$ qualité, avec nœuds, - $4 / 8$;

6 chons, $\quad-1 / 8$;

Ces rapports varient avec les dimensions des piè- 
ces, et selon que la tronce renferme plus ou moins de nœuds; le rendement lui-même est, sinon plus fort en volume, toujours plus avantageux sous le rapport de la valeur commerciale, quand la tronce est assez forte pour fournir de la planche large.

Pour qu'une tronce de sapin puisse être débitée en planches marchandes sans trop de déchet, il faut qu'elle ait au moins $0^{\mathrm{m}}, 38$, ou 14 pouces, de diamètre au petit bout. Mais on utilise le petit bout des gros sapins, de même que les arbres de faibles dimensions (jusqu'à $0^{\mathrm{m}}, 20$ ou 7 pouces de diamètre) en les débitant d'une autre manière : on en fait de petits madriers, des membrures, des frises, des douves, des lattes, etc. - - On peut ainsi tirer un parti avantageux de sapins qui n'étaient employés autrefois que comme bois de feu, ou bien comme pièces de menue charpente quand les bois avaient assez de longueur.

L'unité de vente des sciages de sapin est le cent ou le mille de planches ordinaires ou marchandes.

Nous avons vu que les planches ordinaires ou marchandes, sont celles de 12/9 et quelquefois de 11/9. Mais lorsque l'on dit que les planches valent 115 ou 118 francs, ce prix s'applique à cent planches de 12/9. Les planches de 11,9 valent toujours 9 ou 10 francs de moins par cent que celles de $12 / 9$.

Les planches réduites de $12 / 8$ et $11 / 8$ se paient, 
en moyenne, 20 francs de moins que celles de 9 pouces de large et de même longueur.

Les planches larges de $12 / 12$ et 11/12 se comptent, dans le commerce, pour une planche et demie ordinaire de même longueur, bien qu'elles ne cubent que un tiers en sus.

Dans un lot de sciage, le cent ou le mille de planches contient ordinairement $1 / 4,1 / 3$, ou même moitié de chons et rebuts; mais on ne compte un chon ou un rebut que pour une demi-planche.

Nous avons dit que, dans les Vosges, le sapin, l'épicéa et le pin sylvestre se débitent en planches dans des scieries spéciales. Ces usines appartiennent les unes à l'Etat, les autres aux communes propriétaires de forêts ou à des particuliers, et sont établies, à proximité des sapinières, sur les nombreux cours d'eau qui sillonnent les vallées. Les scieries de l'Etat sont affectées au service des coupes vendues dans les forêts domaniales, moyennant certaines conditions d'entretien imposées aux adjudicataires par le cahier des clauses spéciales. Il en est de même des scieries communales; mais comme ces usines ne sont pas toujours en nombre suffisant pour effectuer le sciage de tous les bois, dans le délai accordé aux adjudicataires, une partie souvent assez considérable des tronces de sciages est débitée dans des scieries particulières, au prix moyen de 80 francs le mille de planches. Dans l'état actuel de ces usines, une bonne scierie à bloc débite, au maximum, 30000 planches par an; une scierie à manivelle 45000 planches. 
Nous avons déjà vu que le débit du sapin diffère d'une région à une autre. Dans le Jura, où la traite des bois est souvent plus facile que dans les Vosges, on expédie dans leur entier des bois qui seront sciés en planches de grandes longueurs sur les marchés, à Beaucaire ou ailleurs. On y fait aussi, sur place, des planches brutes ou planches de plat, suivant le débit de Strasbourg, et des planches aligrées, c'està-dire lavées sur les bords comme celles des Vosges. On n'emploie que des bois de choix dans la fabrication des planches brutes; et on leur donne une épaisseur de $0^{\mathrm{m}}, 027,0^{\mathrm{m}}, 03^{\mathrm{m}}, 0^{\mathrm{m}}, 040$, ou 12,15 , ou 18 lignes. On les vend à tant le mètre de largeur, celle-ci étant mesurée au milieu de la longueur et du côté le plus étroit pour chaque planche.

On donne aux planches alignées jusqu'à 5 mètres de longueur et une largeur en pouces qui varie de 4 à 12 ; elles se vendent souvent à la douzaine.

On fabrique en outre des sciages plus minces, appelés lambris, qui n'ont que $0^{\mathrm{m}}, 014,0^{\mathrm{m}}, 018$ ou $0^{\mathrm{m}}, 020$ d'épaisseur, 6,8 ou 9 lignes. Non alignés ils se vendent, comme les planches brutes, au mètre de largeur; alignés, à la douzaine.

La bille de sciage, qui a généralement 4 mètres de longueur, est appelée plot. Un plot de $0^{\mathrm{m}}, 50$ de diamètre au petit bout peut donner 10 planches brutes de $0^{\mathrm{m}}, 040$, ou 11 de $0^{\mathrm{m}}, 034$ d'épaisseur; de sorte que le déchet en dosseaux est très-faible.

Les planches de sapin qui alimentent le commerce 
de Paris proviennent, pour la plus grande partie, des Vosges. Celles que l'on fabrique dans le Jura et les Alpes, se déversent plus spécialement dans le bassin du Rhône et de la Saône et dans celui de la Loire. Les sapinières des Pyrénées (1) fournissent leurs produits aux grands centres de consommation du Roussillon et du Languedoc. Les provinces de l'Ouest, surlout celles qui avoisinent l'Océan, s'approvisionnent plus particulièrement dans les ports de mer où affluent les produits forestiers du nord de l'Europe. Cet état de chose semble devoir se modifier depuis que les transports ont été rendus plus faciles, et Paris tend à devenir le principal marché de ce genre de produits, aussi bien pour les bois du Jura, des Alpes et du nord de l'Europe que pour ceux des Vosges.

Sur tous les marchés principaux, les planches de sapin, d'épicéa ou de pin, sont appelées indistinctement planches de sapin, mais on les distingue les unes des autres d'après la couleur du bois. Celles du sapin et de l'épicéa sont désignées sous le nom de bois blanc ou sapin blanc; celles du pin sylvestre

(1) La difficulté des transports (ils ne peuvent s'effectuer le plus sonvent que par traînage) oblige à découper les bois de charpente on de sciage à des longueurs beaucoup moindres que celles qu'on pourrait leur donner, s’il existait des chemins de vidange en bon état. Ainsi dans les sapinières du département de l'Aude, on ne donne que 4 à 12 mètres de longueur aux pièces de charpente, et $2^{\mathrm{m}}, 50$ seulement aux tronces de sciage. C'est un état de chose qu'il serait bien important de faire cesser, surtout à cause de la qualité remarquable et tout à fait exceptionnelle des sapins de celte contrée. 
sont dites de bois rouge ou de sapin rouge. Au point de vue de la qualité du bois, il importe de faire une différence entre les unes et les autres, surtout dans les emplois qui exigent de la solidité. Sous ce rapport, les planches de sapin et d'épicéa offrent à peu près les mêmes qualités, et sont de beaucoup inférieures aux planches de pin sylvestre, quand cellesci sont purgées d'aubier. Mais ce qui influe surtout sur la solidité et la durée de ces planches, lorsqu'elles sont mises en œuvre dans la construction des parquets ou planchers par exemple, c'est la manière dont le bois a été débité. Une planche de sapin, d'épicéa, ou de pin sylvestre fait un bien meilleur usage quand elle a été débitée dans le sens des rayons médullaires, que lorsque le sciage a eu lieu dans la direction contraire; elle est de plus bien moins sujette à se déjeter ou à prendre du retrait dans tous les ouvrages de menuiserie où elle peut être employée. Le pin du Nord, dont on fait une grande consommation dans l'ouest de la France, dure au moins aussi longtemps que le chêne, quand il a été scié sur maille, et il a de plus une couleur jaune rosé, d'un très-joli effet dans l'emploi de ces bois en planches à parquet.

Les peupliers fournissent aussi beaucoup de sciages à la consommation. On en fait des planches, et surtout de la volige un peu plus épaisse que celle de chêne. Ces planches s'emploient à l'intérieur des meubles, à la fabrication des caisses d'emballage et, 
en général, aux mêmes usages que les sciages de sapin. C'est un bois qui ne se tourmente pas lorsqu'il est mis en œuvre, même sans être desséché. Cette propriété le rend précieux en ébénisterie où on l'emploie beaucoup pour faire la carcasse de meubles destinés au placage, et en menuiserie pour faire des panneaux de porte ou de lambris, et même des planchers. Mises en œuvre à couvert et dans un lieu sec, les planches de peupliers font un très-bon usage, surtout quand elles sont recouvertes d'une couche suffisante de peinture à l'huile ou d'encaustique. - On les tire d'ailleurs en bien plus grand nombre des arbres venus isolément que des forêts.

\section{SIV. - Bois de fente.}

Outre les sciages, on façonne encore dans' les coupes beaucoup d'autres bois d'industrie, que l'on désigne sous le nom général de bois de fente. On débite les bois de fente sous différentes formes et sous des dimensions très-diverses, selon les usages auxquels ces bois sont destinés. Ce travail se fait surtout en forêt, parce que le bois se fend beaucoup mieux et plus facilement, lorsqu'il est vert et tout saignant, que lorsqu'il est desséché; parce qu'aussi tous les bois ne sont pas propres à la fente, et que dans les coupes en exploitation, l'ouvrier a plus de facilité pour choisir le bois qui convient à ce genre de débit; parce qu'enfin il peut souvent employer à des ouvrages de fente des pièces de fausse coupe, et 
tirer ainsi le parti le plus utile des bois. Pour qu'un bois soit propre à la fente, il faut qu'il ait une texture égale, que ses fibres soient parfaitement droites, apposées régulièrement les unes contre les autres, et qu'il soit exempt de nœuds, de vices et de tout autre accident de croissance.

Les essences qui fournissent le plus de bois de fente sont le chêne, le hêtre, le sapin et l'épicéa.

Parmi les chênes de France, le rouvre occupe le premier rang comme bois de fente. Le pédonculé est plus noueux, surtout quand il n'a pas été élevé en massif plein; sa fibre est ordinairement plus serrée, son grain plus fin, son bois plus nerveux et plus coriace, toutes circonstances qui le rendent plus difficile à fendre sous le coutre de l'ouvrier. Le bois du chêne tauzin, de l'yeuse et du chêne liége est plus dur encore que celui du pédonculé; il a de plus i'inconvénient de se gercer profondément et de se déjeter en se desséchant, ce qui le rend tout à fait impropre à ce genre de débit.

Entre tous les métiers qui emploient les bois de fente, il faut placer en première ligne la tonnellerie. On n'emploie guère que du chêne, purgé de tout aubier, pour la fabrication des futailles qui doivent contenir du vin et de l'eau-de-vie, parce que les fibres de son bois sont mieux liées, plus serrées et moins perméables. Cependant on fait aussi de bonnes futailles avec le jeune châtaignier et, dans le Midi, on emploie quelquefois à cet usage le bois de 
mûrier blanc; mais le bon chêne est préférable. Le frêne est spécialement employé pour la construction des tonneaux destinés à contenir du kirsch, à cause de la propriété dont il jouit de ne point altérer la couleur du liquide.

On nomme merrain le bois de fente destiné plus particulièrement à la fabrication des douves de tonneaux. On le débite dans les coupes de la manière suivante. La biile à merrain étant sciée à la longueur convenable, on la fend d'abord en gros quartiers dont chacun se divise ensuite en deux, trois ou quatre secteurs. L'ouvrier fendeur travaille alors chacun des secteurs séparément avec un instrument appelé coutre, formé d'une forte lame adaptée à angle droit à un manche assez court. Il enlève le cœur du bois, dont la forme aiguë ne se prête pas à la division en merrains, et il supprime tout l'aubier. Il fend ensuite le restant du secteur, suivant des lignes perpendiculaires au rayon du milieu, en prismes d'épaisseur convenable, et dont le nombre dépend de la grosseur de la bille. Enfin chacune de ces pièces se divise, suivant la direction des rayons, en plancheltes de merrain, qui sont ensuite parées à la plane. On transporte le merrain préparé de la sorte dans les ateliers de tonnellerie où il est mis en œuvre.

Les douves sont de deux sortes; celles qui servent pour construire le corps du tonneau et que l'on désigne sous différents noms tels que longailles, douelles, merrains, passe-rebuts, etc., et celles qui sont employées pour former les fonds du tonneau et que 
I'on nomme foncailles, fonds ou traversins. Dans la fabrication du merrain la longueur et l'épaísseur des deux échantillons principaux sont déterminées d'avance d'áprès les dimensions des futailles à construire, mais leur largeur est variable, au moins dans une certaine mesure, et peut descendre jusqu'à la moitié de celle des types correspondants. Ces pièces de largeur réduite reçoivent différents noms, selon les localités ; on les appelle tricages dans les Vosges, ganivelles ou rebuts dans le centre de la France.

Dans le commerce, un lot de merrains assortis se compose de $2 / 3$ de douelles et $1 / 3$ de fonds. L'unité de vente est le millier, lequel comprend, dans chaque localité, le nombre de douves assorties nécessaire pour construire une quantité déterminée de tonneaux d'une capacité donnée. Mais, pour une même contrée, on fabrique souvent des merrains de dimensions variables; c'est ainsi que pour la Bourgogne on fait du merrain pour des pièces de 228 litres et pour des feuillettes de 114 litres. De sorte que dans l'assortiment du merrain fabriqué pour les besoins de cette contrée, il faut faire entrer, dans une certaine proportion, les diverses espèces de douves qui servent à la construction de ces deux genres de tonneaux. En outre, on comprend dans chaque millier une certaine quantité de douves de largeur réduite, ou tricages, dont la valeur s'apprécie en fonction du type auquel ces pièces se rapportent. 
Dans les Vosges (forêt de Darney), on fabrique plus spécialement du merrain pour la Bourgogne, et on idonne le nom de marquandise aux douves pour feuillettes, afin de les distinguer de celles destinées à construire des pièces. Les dimensions de toutes ces douves sont les suivantes :

Bonne douelle, long. $0^{\mathrm{m}}, 88$, larg. $0^{\mathrm{m}}, 11$ à $0^{\mathrm{m}}, 15$, épais. $0^{\mathrm{m}}, 027$ Tricage de douelle, $\quad 0,88,-0,06$ à $0,10,-0,027$ Bon fond, $\quad-0,66,-0,14$ à $0,19,-0,027$ Tricage de fond, $\quad-0,66,-0,08$ à $0,1 \overline{5},-0,027$ Douelle, marquandise, - $0,77,-0,06$ à $0,10,-0,025$ Fond, - - $\quad-0$, ,0, - 0,08 à $0,13,-0,023$

Le millier des Vosges, complétement assorti, comprend 4,654 pièces qui se répartissent et s'estiment de la manière suivante (1):

Bonnes douelles.... 856, comptées à raison de 107 p. $0 / 0$ pour 800

Tricages de donelles.. $632 \quad-\quad 1601 / 2 \quad-400$ Bons fonds ....... $428 \quad-\quad 107 \quad-400$

Tricages de fonds... $521 \quad-\quad 1601 / 2 \quad-200$

Douelles, marquandise $1605 \quad-\quad 2671 / 2 \quad 600$ Fonds,

$\frac{802}{4634}$

$2671 / 2-\frac{500}{2700}$

Le millier des Vosges, vendu sans marquandise et réduit comme précédemment, se compose de 3,370 pièces, savoir :

(1) Il est d'usage, dans celte contrée, d'ajouter à chaque centaine de douves 7 pièces dites de rhabillage, ou de donner 7 p. $0 / 0$ de fourniture; de sorte qu'il faut 107 pièces de bonnes douelles ou de bons fonds pour faire le cent réduit, $1601 / 2$ de tricage au lieu de 150, et 267 1/2 de marquandise au lieu de 200. 
Bonnes douelles........ 1284 qui comptent pour 1200

\begin{tabular}{|c|c|c|c|}
\hline Tricages de douelles ...... & 963 & - & 600 \\
\hline Bons fonds............ & 642 & - & 600 \\
\hline Tricages de fonds........ & 481 & - & 300 \\
\hline
\end{tabular}

Le millier des Vosges comprend ainsi toujours 27 cents. - Avec le millier, sans marquandises, on peut fabriquer 100 pièces de Bourgogne, de 228 litres, et avec le millier complétement assorti 67 pièces de 228 litres et 66 feuillettes de 114 litres, soit encore l'équivalent de 100 pièces. Mais quand les largeurs sont bonnes, le millier peut donner jusqu'à 110 pièces.

Le volume du millier de merrain fabriqué est approximativement de huit mètres cubes; et il faut de 13 à 24 mètres cubes de bois en grume pour fournir un millier de merrain. Le déchet dû à la fabrication du merrain des Vosges est donc de 40 à 66 p. 0/0. On conçoit du reste que ce déchet varie avec les forêts, qu'il dépend surtout de la quantité d'aubier que renferme le bois, et aussi de la dimension des arbres et de leur aptitude à la fente. Le prix du merrain des Vosges a varié pendant ces dernières années, avec l'abondance des récoltes, entre 540 et $1100 \mathrm{fr}$. le millier.

Dans le pays de Blois, où la fabrication du merrain emploie presque tous les chênes livrés à l'exploitation, le millier de merrain se compose de 4350 pièces, et comprend le nombre de douves as- 
sorties nécessaire pour la fabrication de cent tonneaux de 228 litres. Ces douves sont de six sortes; elles ont toutes la même épaisseur, $0^{\mathrm{m}}, 014$ à $0^{\mathrm{m}}, 015$; on les estime en fonction d'un type qui est le fond marchand, et on les répartit, dans la composition du millier, ainsi qu'il suit :

$1^{\circ} 200$ fonds marchands qui ont $0^{\mathrm{m}}, 66 \mathrm{de}$ longueur, et $0^{\mathrm{m}}, 14$ à $0^{\mathrm{m}}, 20$ de largeur, comptent pour. . . . . . . . .

$2^{\circ} 900$ ganivelles, qui ont $0^{\mathrm{m}}, 66$ de longueur et $0^{\mathrm{m}}, 06$ à $0^{\mathrm{m}}, 14$ de largeur, comptent pour. . . . . . . . . .

$3^{\circ} 300$ chanteaux (1), qui ont $0^{\mathrm{m}}, 50 \mathrm{de}$ longueur et $0^{\mathrm{m}}, 08$ à $0^{\mathrm{m}}, 18$ de largeur, comptent pour. . ....... 100

$4^{\circ} 450$ passe-rebut, qui ont $0^{\mathrm{m}}, 84$ de longueur et $0^{\mathrm{m}}, 14$ à $0^{\mathrm{m}}, 20$ de largeur, comptent pour.

$5^{\circ} 2200$ rebuts, qui ont $0^{\mathrm{m}}, 84$ de longueur et $0^{\mathrm{m}}, 05$ à $0^{\mathrm{m}}, 14$ de largeur, comptent pour 1,100

$6^{\circ} 300$ petits rebuts, qui ont $0^{\mathrm{m}}, 84$ de longueur et $0^{\mathrm{m}}, 05$ de largeur comptent pour. .

Ce qui fait 4350 pièces qui comptent pour $\overline{2,100}$ On fait encore dans les forêts du Blésois du merrain spécial pour le Bordelais; le millier de merrain Bordelais ne comprend que quatre sortes de douves ayant une épaisseur commune de $0^{\mathrm{m}}, 016$; ce sont :

(1) On nomme chanteau une pièce ordinairement moins large que la fonçaille et qui se place en travers et en dehors des fonds de tonneau pour les consolider. 
DES BOIS DE TRAYAIL OU D'INDUSTRIE.

Le fond marchand qui a $0^{\mathrm{m}}, 69$ de long. sur $0^{\mathrm{m}}, 16$ a $0^{\mathrm{m}}, 20$ de larg.

Le chanteau

Le passe-rebut - $0,84 \quad-\quad 0,11$ à $0,20 \quad-$

Le rebut

- $0,84 \quad-\quad 0,06$ à $0,11 \quad$

Le volume du millier de merrain ordinaire de Blois est approximativement de $4^{\text {m.c. }}, 000$; il faut de 7 à 8 mètres cubes de bois en grume pour fournir un millier de merrain fabriqué.

En Champagne, le merrain se vend à la treille. La treille se compose d'un nombre déterminé de longailles, de fonçailles et de chanteaux, qui représentent le bois nécessaire à la construction de 50 tonneaux de 200 litres. On estime qu'il faut en moyenne 3 mètres cubes au $1 / 4$ sans déduction, ou un peu moins de 4 mètres cubes en grume, pour donner une treille de merrain.

Dans les poudreries de l'Etat, on construit des chapes ou tonneaux destinés à loger les poudres, et pour lesquels on emploie du merrain de chêne de dimensions variables. L'adjudication de ces fournitures se fait au mille de pièces de longueur et d'épaisseur déterminées, et dont la largeur, variable pour chaque pièce, doit atteindre un certain minimum fixé d'avance pour chaque dizaine de pièces. Ainsi, par exemple, 10 longailles pour chapes de 100 kil. doivent avoir une largeur totale de $1^{\mathrm{m}}, 30$.

La marine emploie du merrain assorti de trois espèces pour la construction des tonneaux ou des 
pièces dites de 4 , de 3 et de 2. Le millier assorti des trois espèces est de mille longailles et de six cents fonçailles ou de quatorze cents longailles.

Ces exemples suffisent pour faire voir combien la fabrication du merrain est variable suivant les contrées. Pour qui en suit les développements, il est facile de constater aussi que l'on donne une épaisseur bien moindre aux douves de toute sorte à mesure que les chênes, les gros chênes surtout, deviennent plus rares et se vendent plus cher. C'est que cette fabrication n'emploie que des bois de choix et suffirait à elle seule pour absorber une grande partie des chênes que la France produit, si nos viticulteurs ne trouvaient dans l'importation du merrain étranger le complément des produits nécessaires à leur industrie (1).

Avec le chêne on fabrique aussi des échalas en quantité considérable, des lattes de plafonneur, des rais de voiture pour les charrons, des tringles d'espalier, des gournables pour la marine et des cerches. A l'exception des gournables qui exigent du bois de première qualité, et des cerches pour la confection desquelles on ne peut employer que des chênes de forte dimension et de la plus belle fente, tous ces ouvrages se fabriquent avec des bois trop faibles pour être débités en sciages ou en charpente,

(1) En 1865, on a importé en France du merrain d'Autriche pour une valeur de plus de $25,000,000$ de francs. 
DES BOIS DE TRAVAil OU D'INDUSTRIE.

ou avec des chutes et des pièces de fausse coupe. Tous ces produits se vendent par bottes dont chacune renferme une quantité déterminée d'échalas, de lattes, etc., suivant les usages de chaque contrée.

On fait encore du merrain avec du bois poreux tel que le sapin, l'épicéa, le peuplier; mais ces douves ne sont propres qu'à construire des tonneaux destinés à recevoir des marchandises sèches.

Avec le sapin et l'épicéa on fait des essandoles, ou bardeaux pour la couverture des maisons, du merrain pour la fabrication des seaux, cuves et cuveaux, des cerches minces pour les boîtes à fromage et autres menus ouvrages de fente. Les bois que l'on débite de la sorte doivent être exempts de nœuds, parfaitement sains et de bonne fente. Ils se vendent au mètre cube et en grume, et leur prix varie suivant celui de la planche ou de la marchandise qui a le cours le plus régulier dans la localité.

Comme bois de fente, le hêtre sert à une foule d'emplois que l'on désigne d'une manière générale sous le nom d'ouvrages de râclerie. Tels sont les cerches dont on fait des chaserets ou clayettes pour fromages; les éclisses dont on fabrique des boîtes très-légères pour les confiseurs, pharmaciens, parfumeurs, bijoutiers, etc., des seaux et autres mesures de capacité, des jouets d'enfants, etc.; les lattes pour fourreaux d'épée et de sabre; les copeaux pour la 
gaînerie, la miroiterie, etc.; les panneaux de soufflet, les battoirs à lessive, les pelles à four, les bois de brosses. On fabrique aussi avec le hêtre des attelles de collier, des bâts, sellettes et arçons; des semelles de galoche et des sabots; des rames, écopes et avirons.

Pour tous ces ouvrages, le bois doit être de fente facile et droite, et, quand il doit être débité en planches très-minces telles que des cerches, il faut de plus que sa fibre soit à la fois souple, forte et élastique, qualités que l'on ne trouve ordinairement réunies que dans les arbres d'àge moyen, 80 à 100 ans.

Le déchet qui résulte de la confection des ouvrages de râclerie varie entre 50 et 75 p. $0 / 0$; mais les débris sont recueillis et employés au chauffage.

Toutes ces industries n'emploient, relativement, qu'une quantité assez minime de bois de hêtre, sauf cependant celle des sabotiers qui, dans certaines localités, a quelqu'importance. La saboterie a pris plus d'extension depuis quelque temps, par suite de l'emploi de machines à saboter qui fabriquent plus vite et mieux que les ouvriers ordinaires, et de la facilité actuelle d'exporter les produits dans des contrées éloignées des lieux de production (1).

Les sabots se vendent ordinairement à la douzaine

(1) Plusieurs usines à saboter de l'Alsace ont pour principaux débouchés de leurs produits l'Amérique et l'Algérie. 
DES DOIS DE TRAVALL OU D'INDUSTRIE.

de paires, à la grosse de 144 paires, et à la somme de 80 paires. Chaque unité, douzaine, grosse ou somme, contient un tiers de sabots d'hommes, un tiers de sabots de femmes, et un tiers de sabots d'enfants. On calcule qu'un mètre cube de bon bois de fente peut fournir, en moyenne, 100 à 110 paires de sabots assortis. 


\title{
CHAPITRE QUATRIEME.
}

\author{
BOIS DE MARINE.
}

ARTicle I.

Classement, mesurage et cubage des bois de chêne.

S I. - Exercice du martelage dans les forêts domaniales.

Autrefois, le département de la Marine faisait rechercher et marquer par ses Agents les chênes propres aux constructions navales qui devaient tomber dans les coupes à exploiter chaque année dans les forêts de l'Etat, des communes, des établissements publics et des particuliers. Le prix de ces bois, sur place, était réglé de gré à gré et, en cas de contestation, par des experts nommés contradictoirement. Primitivement, ce privilége avait été reconnu à la Marine par une suite d'Ordonnances royales, dont la plus ancienne paraît remonter à 1318; mais l'exercice de ce droit n'a été sérieusement réglementé que par l'Ordonnance de 1669. L'ordonnance royale du 8 août 1816 , le Code forestier et l'Ordonnance du $1^{\text {er }}$ août 1827 n'ont fail que reproduire et consacrer les dispositions de l'Ordon- 
Classement, mesurage et cubage des boIS DE chêne. 103 nance de 1669 , en ce qui concerne le martelage de la marine. Le département de la Marine a donc pu exercer, pendant de longues années, un véritable droit de préemption sur tous les bois de chêne, propres à son usage, que l'Etat, les communes et les particuliers désignaient chaque année à l'exploitation; mais l'exercice de ce droit était entouré de telles difficultés que la Marine avait renoncé, en 1838, à user de son privilége, sauf à y revenir ultérieurement, si cela devenait nécessaire. Depuis lors, le Ministre de la Marine a mis en adjudication publique les fournitures de bois à faire dans les arsenaux pour lo service des constructions navales; mais, après un essai qui a duré 20 ans, il a paru utile de revenir à l'exercice de l'ancien martelage, en le modifiant toutefois conformément au Décret du 16 octobre 1858, que nous allons rapporter.

DÉCRET DU 16 OCTOBRE 1858.

ARTICLE PREMIER.

Notre Ministre des Finances est autorisé à faire réserver et livrer directement, chaque année, par l'Administration des forêts à la marine impériale, les bois extraits des forêts dépendant du domaine de l'Etat et propres aux constructions navales, en se conformant aux prescriptions ci-après :

ART. 2.

Chaque année, avant le $\mathbf{1}^{\text {er }}$ février, la Direction générale des forêts fera connaître au Ministre de la Marine, par départements 
et arrondissements, les forêts domaniales renfermant des arbres de marine et dans lesquelles des coupes devront avoir lieu.

Il sera accusé réception de ce document par le département de la Marine qui, dans le délai d'un mois, sera tenu d'indiquer à la Direction générale des forêts, par départements et arrondissements, les coupes dans lesquelles la Marine désirera que des arbres lui soient réservés. A cet étal en sera joint un autre donnant le détail des espèces et signaux dont les constructions navales auraient plus spécialement besoin, et des espèces et signaux qu'il serait au contraire inutile de comprendre dans le martelage.

\section{ART. 3.}

Les arbres réservés pour la marine impériale porteront l'empreinte d'un marteau spécial et d'un numéro de série appliqués par les agents de l'Administration des forêts.

Cette Administration fera dresser de ce martelage un procèsverbal contenant toutes les indications propres à faire juger de l'importance approximative de chaque arbre. Copie de ce procèsverbal dûment certifiée sera transmise à notre Ministre de la Marine.

\section{ART. 4.}

Les arbres réservés pour la marine ne seront compris dans les ventes que pour les houppiers et, en général, pour toutes les parties non réservées dont le détail sera donné sur les affiches des ventes.

Les adjudicataires seront chargés de l'abatage, de l'écorçage et du transport des arbres martelés à un point déterminé de la forêt, dont la distance au centre de chaque vente sera indiquée sur les affiches susdites. 
ART. 5 .

Aussitôt après que les arbres auront été réunis sur les lieux de concentration, l'Administration des forêts en donnera avis aux Ingénieurs de la marine, préposés à la surveillance des fournitures de bois, qui prendront des mesures pour que l'examen des arbres commence dans un délai qui n'excédera pas un mois. Ils informeront l'Administration forestière du jour fixé pour le début des opérations.

ART. 6.

En procédant à cette visite, et afin de ne faire choix que de pièces propres à la construction des navires, la Marine pourra faire ébouter les arbres et en faire sonder les nœuds ou autres défauts, à la hache ou à la tarière.

Dans le cas où les pièces rebutées auraient subi une dépréciation par suite des sondages, il en sera tenu compte au département des Finances.

\section{ART. 7.}

Les pièces dont la Marine aura fait choix seront marquées de son marteau; elles seront ensuite découpées et équarries par ses soins et à ses frais. La Marine ne devra au département des Finances que le prix des pièces équarries, en raison de leur cube et de leur nature par espèce, ce cube étant calculé suivant les procédés de recette de la marine.

Les pièces rebutées, de même que les remanants de toute nature, resteront à la charge de l'Administration des forêts qui en opèrera la vente suivant les formes ordinaires.

\section{ART. 8.}

Un procès-verbal, dressé contradictoirement par l'Ingénieur de la marine et l'Agent forestier, constatera : 
$1^{\circ}$ Le nombre et les dimensions des pièces livrées à la Marine ainsi que l'essence des bois ;

$2^{\circ} \mathrm{La}$ valeur de ces pièces estimées isolément;

$3^{0}$ Le montant de l'indemnité qui pourra être due par la Marine pour la dépréciation causée par les sondages aux pièces rebutées.

Ce procès-verbal contiendra l'avis distinct de l'Ingénieur de la marine et de l'Agent forestier; en cas de désaccord entre eux sur le montant des prix ou des indemnités, il sera dressé en double minute, dont l'une sera adressée au département de la Marine et l'autre à la Direction générale des forêts.

Les bois ne pourront être enlevés par les agents de la marine qu'après la rédaction du procès-verbal sus énoncé.

\section{ART. 9 .}

Une commission nommée par le Ministre des Finances el le Ministre de la Marine sera chargée, chaque année, d'arrêter définitivement le compte des sommes dues par le département de la Marine.

Ces sommes seront payées au département des Finances, selon le mode indiqué par l'article 19 de l'Ordonnance du 31 mai 1838; elles figureront en recette au budgel de l'Administration des forêts.

ART. 10

Nos Ministres secrétaires d'Etat aux départements des Finances et de la Marine sont chargés de l'exécution du présent décret.

Fait au palais de Saint-Cloud, le 16 octobre 1858 .

$$
\text { Signé : Napoléon. }
$$

Les dispositions prescrites par ce Décret ont subi 
plusieurs modifications successives et, à la date du 24 février 1866 , une instruction, concertée entre le Directeur général des Forêts et le Directeur du matériel au Ministère de la Marine, a résumé les règles aujourd'hui en vigueur pour la délivrance des bois de marine dans les forêts de l'Etat. Nous ne rapporterons de ces règles que celles qui intéressent le plus directement l'exercice du martelage, le cubage et la délivrance :

A. Avant l'ẻpoque du balivage des coupes, les Agents de la Marine désignent, au moyen d'un ceinturage à l'huile, tous les arbres situés dans les cantons en tour d'exploitation annuelle ou périodique, et qu’ils jugent propres aux constructions navales.

B. Il est procédé au martelage des arbres de la marine en même temps qu'à la marque des coupes, et par les mêmes Agents.

Parmi les arbres qu'ils jugent à propos d'abandonner à l'exploitation dans l'enceinte de chaque coupe, les Agents forestiers ne marquent, pour la Marine, que ceux préalablement désignés par elle.

Ces arbres sont frappés d'un marteau spécial à $1^{\mathrm{m}}, 33$ du sol, et. sur deux faces opposées.

c. L'adjudicataire de chaque coupe procède à l'abatage des arbres de marine en se conformant aux dispositions du cahier des charges et aux indications des Agents. Les branches de ces arbres font seules partie de la vente à moins qu'elles n'aient été exceptionnellement réservées.

L'adjudicataire en effectue la coupe suivant les indications du chef de cantonnement.

D. Après l'abatage, ces arbres sont examinés par l'Ingénieur de la marine.

Les pièces dont la Marine a fait choix sont marquées de son 
marteau, découpées et équarries sur place par ses soins et à ses frais.

L'Administration forestière est chargée de la vente des rebuts et remanants.

E. Le compte des sommes dues par le département de la Marine à celui des Finances est réglé par unité de volume réel des pièces acceptées et des rebuts, et pour une période de cinq ans.

Les prix doivent être revisés au début de chaque période quinquennale par une commission mixte, siégeant à Paris.

Pour se rendre un compte exact des opérations de la Marine, et pouvoir apprécier l'intérêt qui s'attache à cette partie du service forestier, il est important que les Agents soient en état :

10 De reconnaître si un arbre est propre à donner une pièce de marine;

2. De déterminer, l’arbre étant sur piè, la nature de la pièce qu'il pourra fournir.

Or, les conditions à remplir pour qu'un chêne soit propre à la marine tiennent à la forme de l'arbre, à ses dimensions et à la qualité de son bois.

\$II. - Nomenclature et configuration des pièces de bois de chêne propres aux constructions navales.

En principe, toute pièce de bois de bonne essence et de configuration régulière, quelles que soient ses dimensions, est propre aux constructions navales.

Partant de cette donnée, le département de la Marine a fait construire un tarif qui permet de classer et de recevoir, conformément à l'emploi dont ils 
sont susceptibles, tous les bois de formes régulières et de dimensions quelconques qu'offre la nature. Les pièces comprises dans ce tarif sont groupées en trois catégories :

Les bois droits, pièces tout à fait droites, ou ne présentant que de faibles courbures inférieures à 20 millimètres par mètre de longueur (le plançon peut, par exception, être fortement courbé dans un sens). $(P l .11$.

Les bois courbants, pièces prises dans le fût d'un arbre présentant une courbure minima de 25 millimètres par mètre de longueur ; ils se subdivisent en bois courbants à une courbure, à deux courbures dans un même plan et à deux courbures dans deux plans différents $(P l$. II et $I I I)$.

Les bois courbes, formés par l'insertion d'une forte branche dans le corps de l'arbre sous des angles déterminés $(P l$. III et $P l . I V$, fig. 2).

Chacune de ces catégories est ensuite divisée en signaux et espèces.

On donne le nom de signal aux diverses pièces de chaque groupe dont la forme est déterminée d'une manière précise par l'emploi auquel elles sont propres.

Les pièces de même forme, ou d'un même signal, sont subdivisées en espèces d'après leurs dimensions.

C'est donc la forme des bois qui détermine surtout le classement des pièces de marine en signaux portant des noms différents, tandis que le nom d'espèce sert à distinguer les pièces de même forme, mais de 
dimensions différentes. Pour opérer la division de chaque signal en espèces, on est parti de ce principe que les bois de même espèce, appartenant à des signaux différents, devaient tous avoir une même valeur, basée sur leur degré d'utilité ou de rareté. D'où il résulte, au point de vue commercial, que le caractère principal de l'espèce est de soumettre à un prix commun du mètre cube tous les signaux qu'elle comprend.

Chaque signal est désigné daus les tarifs par la lettre initiale du nom qu'on lui a donné; l'espèce se désigne par un numéro d'ordre placé devant cette initiale. C'est ainsi que le signal Étambot se subdivise en quatre espèces qui sont désignées dans le tarif de la manière suivante :

$1 \mathrm{ET}-108-\frac{50}{44} \ldots .$. Ce qui signifie : Étambot de première espèce, ayant 108 décimètres de longueur et $ّ 0$ sur 44 centimètres d'équarrissage au milieu et au petit bout.

$2 \mathrm{ET}-86-\frac{46}{40} \ldots .$. Ce qui signifie : Étambot de deuxième espèce, ayant.....

$3 \mathrm{ET}-80-\frac{44}{36} \ldots$.

$4 \mathrm{ET}-70-\frac{40}{52} \ldots$.

De même le signal appelé demi-bau se subdivise en trois espèces, 2DB, 3DB, $4 \mathrm{DB}$ qui, en raison de leurs formes et de leurs dimensions ou de leur degré d'utilité et de rareté, ont une valeur, au mètre cube, 
égale à celles des $2^{\mathrm{e}}, 3^{\mathrm{e}}$ et $4^{\mathrm{e}}$ espèces d'étambot, de pièces de quille, etc.

Les dimensions de l'équarrissage sont données, dans le tarif, sous les noms de largeur sur le tour et épaisseur sur le droit.

La largeur sur le tour, ou simplement le tour, exprime la distance qui sépare les deux faces courbes dans les bois qui présentent une courbure.

L'épaisseur sur le droit, ou simplement le droit, exprime la distance qui sépare les deux faces planes dans les mêmes bois.

Par analogie, on a conservé ces dénominations pour les côtés d'équarrissage des bois tout à fait droits, et alors le tour s'entend du plus grand côté d'équarrissage quand la pièce est méplate.

Les signaux tels que la demi-varangue, l'allonge, la guirlande, etc., pour lesquels le tarif n'indique qu'une seule des dimensions de l'équarrissage au petit bout, sont ceux dont la largeur sur le tour peut être moindre au petit bout qu'au milieu. La dimension exigée est celle de l'épaisseur sur le droit, laquelle doit toujours être la même, dans tous les bois courbants, au petil bout qu'au milieu. Il n'y a d'exception à cette règle que pour le jas d'ancre et la pièce de tour dont les deux dimensions peuvent être moindres au petit bout qu'au milieu, et pour la préceinte et le bois à deux bouges dont les dimensions au petit bout sont exactement fixées.

Ces explications suffisent pour faire comprendre les termes et les signes du tarif suivant : 


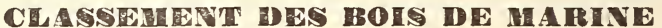

arrêté le 2 juin 1852 par le Ministre de la Marine et modifié par

l'Instruction ministérielle du 8 juin 1859.

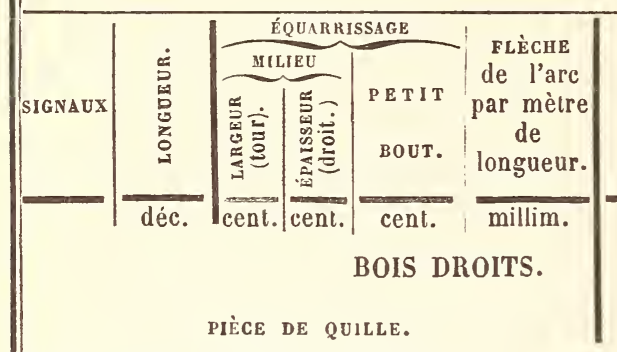

$\left.\begin{array}{|rr|r|r|r|r|}1 & Q^{\prime} & 110 & 44 & 44 & 44-44 \\ 2 & Q^{\prime} & 100 & 40 & 40 & 40-40 \\ 3 & Q^{2} & 90 & 36 & 36 & 56-36 \\ 4 & Q^{\prime} & 80 & 52 & 32 & 32-32 \\ 2 & Q^{2} & 90 & 44 & 44 & 44-44 \\ 3 & Q^{2} & 80 & 40 & 40 & 40-40 \\ 4 & Q^{2} & 70 & 36 & 36 & 36-36\end{array}\right\}$

MÈCHE DE GOUVERNAIL.

$\left.\begin{array}{|r|r|r|r|r}1 \text { MG } & 100 & 72 & 66 & 40-44 \\ 2 \text { MG } & 96 & 64 & 62 & 36-40 \\ 3 \text { MG } & 90 & 56 & 54 & 32-56 \\ 4 \text { MG } & 80 & 46 & 44 & 28-52\end{array}\right\}$

BITTE.

$$
\left.\begin{array}{ll|l|l|l|l}
\text { 马 } & \text { BI } & 46 & 42 & 42 & 38-58 \\
6 & \text { BI } & 40 & 36 & 36 & 30-50
\end{array}\right\}
$$

\begin{tabular}{|c|c|c|c|c|}
\hline $2 \mathrm{P}$ & 110 & 40 & 40 & $56-36$ \\
\hline z p & 100 & 34 & 34 & 30.30 \\
\hline & 100 & 36 & 32 & 52-28 \\
\hline $4 \mathrm{P}$ & 90 & 30 & 30 & $26-26$ \\
\hline & & $\begin{array}{l}32 \\
26\end{array}$ & $\begin{array}{l}28 \\
26\end{array}$ & $\begin{array}{l}28-24 \\
22-22\end{array}$ \\
\hline & 70 & 28 & 24 & $24-20$ \\
\hline $6 \mathrm{P}$ & รั0 & 22 & 22 & $20-20$ \\
\hline
\end{tabular}

La pièce sera tout à fait droite et sans défournis. - Le minimum, au petit bout, sera susceptible de tolérance sur le tour, pourvu que le défourni n'existe que sur une des faces et seulement sur une longueur égale au 1/6 de la longueur totale. - Ce signal exclut tous les bois affectés de défauts qui seraient de nature à occasionner des voies d'eau; tels sont, dans une certaine mesure, les gerçures, gélivares, cadranures, roulures, fibres torses, etc. 1

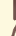

Ce signal est assujetti aux mêmes conditions que la pièce de quille, sauf qu'il n'est pas toléré de défourni au petit bout.

La pièce sera tout à fait droite et sans défournis. - Les largeur et épaisseur sont \{ prises à deux mètres du pied. - Ce signal exclut particulièrement les bois à fibres torses.

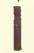

La pièce sera tout à fait droite et sans $\{$ défournis.

1

Les plançons des cinq premières espèces pourront être courbes sur les deux faces, pourvu que la flèche d'une des deux courbures ne dépasse pas 12 millimètres par mètre de longueur. Chaque courbure isera bien suivie et dans le même sens. Ceux de la $6^{\mathrm{e}}$ espèce seront droits sur les deux faces. - Ce signal exclut les bois affectés de défauts qui ne permettraient pas le débit en bordages. 


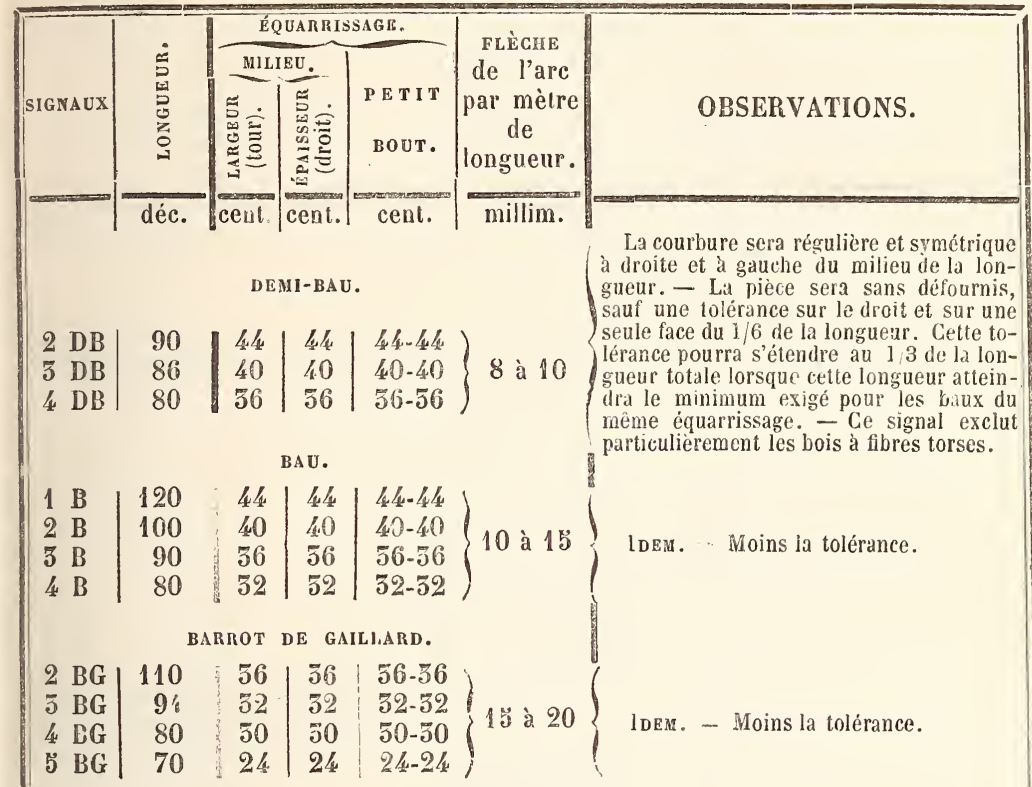

\section{BOIS COURBANTS.}

\section{Rois à une courloure.}

JAS D'ANCRE.

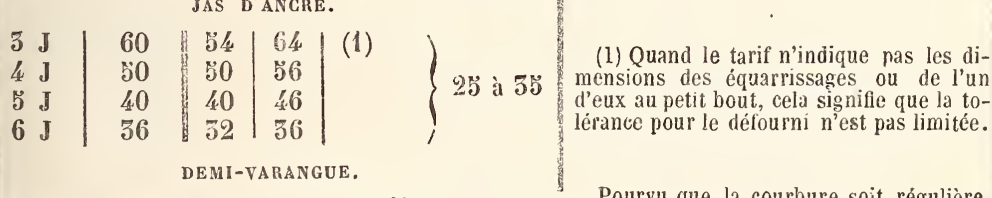

$\left.\begin{array}{ll|l|l|l|l|c|c}5 & \mathrm{DV} & 60 \\ 4 & \mathrm{DV} & 50 & 40 & 50 & -40 \\ 5 & \mathrm{DV} & 40 & 40 & \mathbf{5 6} & -58 \\ 40 & -56\end{array}\right\} \begin{gathered}53 \\ \text { et } \\ \text { au-dessus. }\end{gathered} \quad \begin{aligned} & \text { Pourvu que la courbure soit régulière, } \\ & \text { il n'est pas nécessaire qu'elle soit symétri- } \\ & \text { longueur. }\end{aligned}$
BOUT D'ALLONGE.

$$
\left.\begin{array}{ll|l|l|l|l}
6 & \mathrm{BA} & \mathbf{3 6} & 52 & 28 & -28 \\
7 & \mathrm{BA} & 26 & 22 & 22 & -22
\end{array}\right\} \begin{gathered}
53 \\
\text { et } \\
\text { au-dessus. }
\end{gathered}
$$
VARANGUE PLATE.

\begin{tabular}{|ll|l|l|l|l|l|}
2 & $\mathrm{~V}$ & 80 & 48 & 40 & -40 & \\
3 & $\mathrm{~V}$ & 70 & 44 & 36 & -36 & 35 \\
4 & $\mathrm{~V}$ & 60 & 40 & 32 & -32 & et \\
$\mathrm{g}$ & $\mathrm{V}$ & 50 & 56 & 28 & -28 & au-dessus. \\
6 & $\mathrm{~V}$ & $\mathbf{4 6}$ & 32 & 24 & -24 &
\end{tabular}




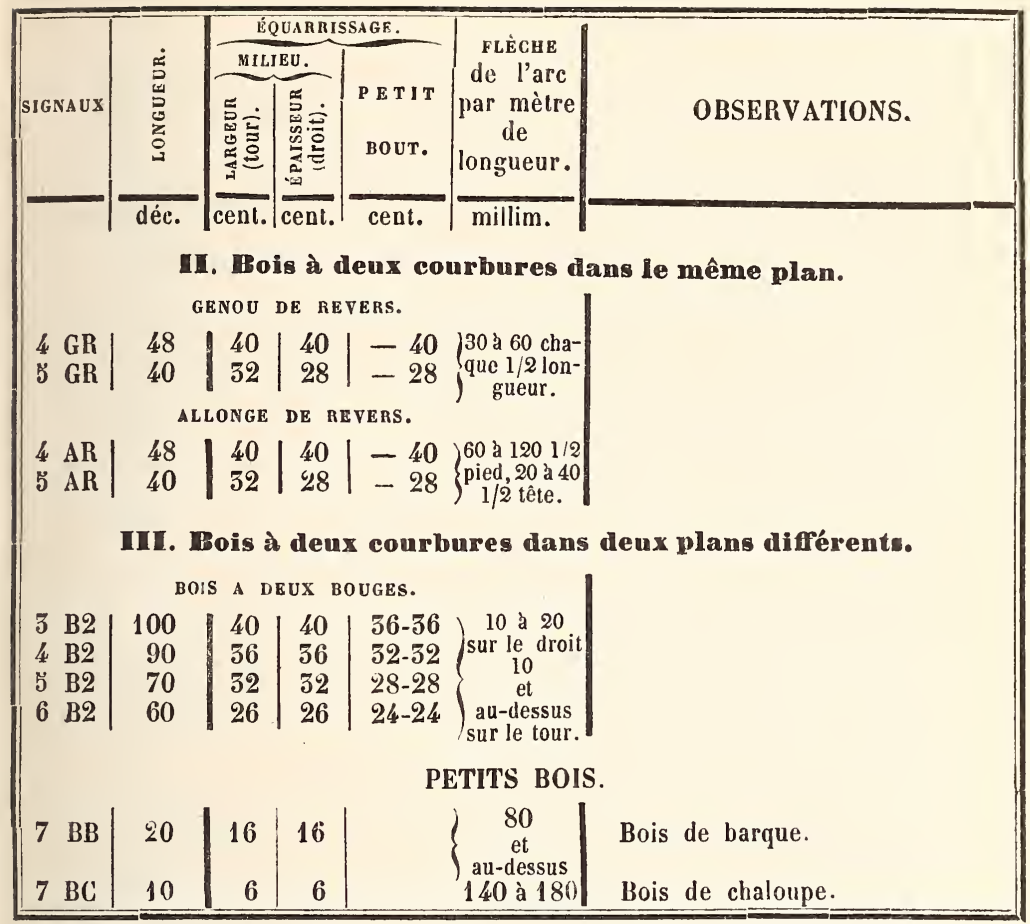

\section{COURBES.}

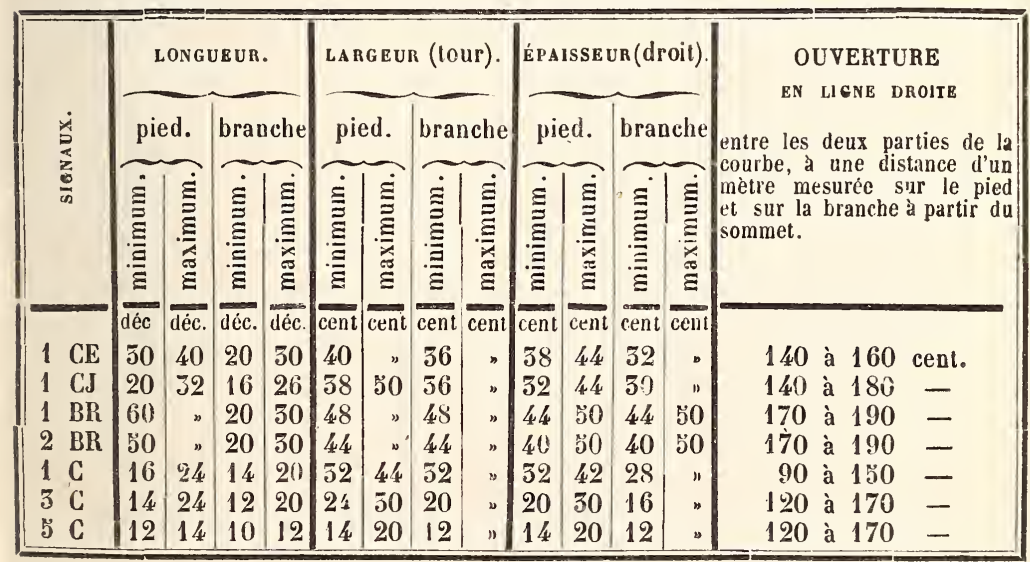


SIII. - Hode de mesurage, de cubage et de classement des bois de marine abatlus, équarris ou en grume.

Les pièces de marine se mesurent différemment selon les formes qu'elles affectent, mais les dimensions s'expriment, d'une manière uniforme, en nombre pair de décimètres pour la longueur et de centimètres pour les côtés de l'équarrissage. Toute fraction d'un décimètre et au-dessous ou d'un centimètre et au-dessous est négligée dans les mesurages; celle qui dépasse un décimètre ou un centimètre compte pour deux.

Pour les bois courbants, le mesurage de la longueur se fait suivant l'arc des pièces ; l'arc se mesure suivant la courbure naturelle des arbres.

Le degré de courbure s'obtient en figurant la corde de l'arc à l'aide d'un ruban, en mesurant la flèche maxima et en la divisant par la longueur. La courbure s'exprime en millimètres de flèche par mètre de longueur de la pièce $(P l . I V$, fig. 4).

Dans les bois courbes, les longueurs du pied et de la branche se mesurent à partir d'un sommet déterminé par la rencontre de deux lignes droites tracées par les milieux des largeurs sur une des faces latérales de la courbe. L'ouverture de la courbe est la distance entre deux points marqués sur ces lignes à 1 mètre du sommet.

Lorsqu'il s'agit de cuber une pièce de marine, les dimensions de l'équarrissage se prennent au 
milieu de la longueur, ou se déduisent, par une moyenne, des équarrissages mesurés sur deux points également distants du milieu. On fait de même à l'égard des courbes, en considérant séparément le pied et la branche.

Quant à la manière de procéder à ce mesurage, l'instruction ministérielle du 8 juin 1859 et le cahier des charges du 4 août de la même année stipulent : que l'aubier et les défournis ou fl. ches existant aux angles des pièces ne donneront liea à aucune réduction, lorsqu'ils n'excèderont pas, à chaque angle, quinze pour cent de la largeur de la pièce à l'endroit correspondant à l'aubier et au défourni, et lorsque d'ailleurs les faces seront saines et sans défectuosités. L'aubier ou le défourni se mesurera sur les faces des pièces et non diagonalement. - Or, on démontre que, pour obtenir les dimensions de l'équarrissage avec tolérance de $150 / 0$ d'aubier à chaque angle, il suffit de multiplier le diamètre sur franc bois, à l'endroit où l'on veut mesurer l'équarrissage, par le coefficient 0,82 .

Les côtés d'équarrissage, ainsi déterminés au milieu et au petit bout, serviront également à classer, par signal et par espèce, toute pièce de marine qui appartiendra à la catégorie de celles pour lesquelles le cahier des charges admet la tolérance d'aubier ou de défourni.

Mais si la pièce doit être rangée parmi les pièces de quille, les étambots, mèches de gouvernail, bittes, 
baux, demi-baux, barrots ou étraves, on ne pourra plus la classer, comme signal et comme espèce, que d'après les côtés de son équarrissage, supposé à vive arête, lesquels s'obtiendront en multipliant le diamètre sur franc bois, au milieu et au petit bout, par le coefficient 0,706 .

Par exemple, soit une pièce, équarrie ou non, qui mesure 9 mètres de longueur et $0^{\mathrm{m}}, 52$ de diamètre au milieu. - Si l'aubier a une épaisseur de deux centimètres, le diamètre sur franc bois sera de $0^{\mathrm{m}}, 48$, et les côtés d'équarrissage avec tolérance de flache ou d'aubier seront de $0^{\mathrm{m}}, 48$ $\times 0,82=0^{\mathrm{m}}, 393$, ou $0^{\mathrm{m}}, 40$. Dès lors la pièce sera reçue pour un cube de $0^{\mathrm{m}}, 40 \times 0^{\mathrm{m}}, 40 \times 9^{\mathrm{m}}=$ $1^{\mathrm{me}}, 440$, et pourra être classée comme une deuxième préceinte, $2 \mathrm{PR}$, si elle a assez de courbure et un équarrissage suffisant au petit bout.

Mais si la pièce est droite et peut donner une quille, le côté de son équarrissage à vive arête sera de $0^{\mathrm{m}}, 48 \times 0,706=0^{\mathrm{m}}, 34$ au milieu. - On calculera de mêne le côté de l'équarrissage sur franc bois au petit bout, et, si cet équarrissage atteint $0^{\mathrm{m}}, 32$, la pièce tombera en $4 Q^{\prime}$, ou en quatrième espèce de quille; mais elle sera reçue comme précédemment pour un cube de $1^{\mathrm{m} c}, 440$.

Le tableau suivant donne immédiatement, en fonction du diamètre sur franc bois, le côté de l'équarrissage à vive arête ou du carré inscrit dans le franc bois, le côté d'équarrissage avec 130 0/0 d'au- 
DES BOIS DE CHÊNE.

bier ou de défourni et le cube correspondant pour un mètre de longueur, (1).

(1) Le classement et le cubage des pièces de marine se faisant d'après les dimensions de l'équarrissage mesurées de 2 en 2 centimètres, on a exprimé, dans la table ci-après, les còtés d'équarrissage en nombre pair de centimètres, en négligeant, dans le calcul, les fractions de 1 centimètre et au-dessous, et en comptant pour 2 centimètres les fractions qui dépassẻnı 1 centimètre.

Les volumes portés dans la table ont été calculés d'après la même règle. 


\begin{tabular}{|c|c|c|c|c|c|c|c|}
\hline \multirow{2}{*}{$\begin{array}{l}\text { Diauètre } \\
\text { sur } \\
\text { franc } \\
\text { bois. }\end{array}$} & \multicolumn{2}{|c|}{ ÉQUARRISSAGE } & \multirow{2}{*}{$\begin{array}{c}\text { сове } \\
\text { pour } \\
\text { un mètre } \\
\text { de } \\
\text { longueur. }\end{array}$} & \multirow{2}{*}{\begin{tabular}{|c} 
DIAMÈTRE \\
sur \\
franc \\
bois.
\end{tabular}} & \multicolumn{2}{|c|}{ ÉQUARRISSAGE } & \multirow{2}{*}{$\begin{array}{c}\text { ссве } \\
\text { pour } \\
\text { un mètre } \\
\text { de } \\
\text { longueur. }\end{array}$} \\
\hline & $\begin{array}{c}\text { à } \\
\text { vive } \\
\text { arête. }\end{array}$ & \begin{tabular}{|c|} 
avec \\
1 o p. 0/0 \\
d'aubier.
\end{tabular} & & & $\begin{array}{l}\text { à } \\
\text { rive } \\
\text { arête. }\end{array}$ & $\mid \begin{array}{cc}\text { arec } \\
1 \stackrel{\text { pa }}{0} & 0 \\
\text { d'aubier. }\end{array}$ & \\
\hline $\begin{array}{l}\text { cent. } \\
\mathbf{2 0}\end{array}$ & $\begin{array}{c}\text { cent. } \\
14\end{array}$ & $\begin{array}{c}\text { cent. } \\
16\end{array}$ & $\begin{array}{l}\text { m.c. } \\
0,026\end{array}$ & $\begin{array}{l}\text { cent. } \\
\mathbf{4 5}\end{array}$ & $\begin{array}{c}\text { cent. } \\
52\end{array}$ & $\begin{array}{c}\text { cent. } \\
56\end{array}$ & $\begin{array}{l}\text { m.c. } \\
0,150\end{array}$ \\
\hline 21 & 14 & 18 & $0,0 \overline{2}$ & 46 & 52 & 58 & 0,144 \\
\hline 22 & 16 & 18 & 0,052 & 貝8 & 34 & 58 & 0,144 \\
\hline 23 & 16 & 18 & 0,052 & 88 & 34 & 40 & 0,160 \\
\hline 24 & 16 & 20 & 0,040 & 49 & 54 & 40 & 0,160 \\
\hline 25 & 18 & 20 & 0,040 & 50 & 56 & 40 & 0,160 \\
\hline 26 & 18 & 22 & 0,048 & 51 & 56 & 42 & 0,176 \\
\hline 28 & 20 & 22 & 0,048 & ส2 & 56 & 42 & 0,176 \\
\hline 28 & 20 & 22 & 0,048 & 53 & 58 & 44 & 0,194 \\
\hline $\mathbf{2 9}$ & 20 & 24 & $0,0 \unlhd ั 8$ & 51 & 58 & 44 & 0,194 \\
\hline 30 & 22 & 24 & 0,0 อั8 & $5 \overline{5}$ & 38 & 46 & 0,212 \\
\hline 31 & 22 & 26 & 0,068 & 56 & 40 & 46 & 0,212 \\
\hline 32 & 22 & 26 & 0,068 & 58 & 40 & 46 & 0,212 \\
\hline $\mathbf{3 3}$ & 24 & 28 & 0,078 & 58 & 40 & 48 & 0,250 \\
\hline 34 & 24 & 28 & 0,078 & 59 & 42 & 48 & 0,250 \\
\hline 35 & 24 & 28 & 0,078 & 60 & 42 & 50 & 0,250 \\
\hline 36 & 26 & 50 & 0,090 & $B 1$ & 44 & รั0 & 0,230 \\
\hline 38 & 26 & 50 & 0,090 & 62 & 44 & รั0 & $0,2 \% 0$ \\
\hline 38 & 26 & 52 & 0,102 & 63 & 44 & 52 & 0,270 \\
\hline 39 & 28 & 52 & 0,102 & 61 & 46 & 32 & $0,2>0$ \\
\hline 10 & 28 & 52 & 0,102 & ชี & 46 & $5 \dot{4}$ & 0,292 \\
\hline 1.1 & 28 & 34 & 0,116 & 36 & 46 & 54 & 0,292 \\
\hline 42 & 50 & 54 & 0,116 & 68 & 48 & 54 & 0,292 \\
\hline 48 & 50 & 56 & 0,150 & 68 & 48 & 56 & $0, \overline{3} 14$ \\
\hline 44 & 32 & 56 & 0,150 & 69 & 48 & э6 & 0,514 \\
\hline
\end{tabular}


DES BOIS DE CHENE.

\begin{tabular}{|c|c|c|c|c|c|c|c|}
\hline \multirow{2}{*}{$\begin{array}{l}\text { DIAMÉTRE } \\
\text { sur } \\
\text { franc } \\
\text { bois. }\end{array}$} & \multicolumn{2}{|c|}{ ÉQUARRISSAGE } & \multirow{2}{*}{$\begin{array}{c}\text { cube } \\
\text { pour } \\
\text { un mètre } \\
\text { de } \\
\text { longueur. }\end{array}$} & \multirow{2}{*}{$\begin{array}{c}\text { DIAMÉTRE } \\
\text { Sur } \\
\text { franc } \\
\text { bois. }\end{array}$} & \multicolumn{2}{|c|}{ ÉQUARRISSAGE } & \multirow{2}{*}{$\begin{array}{c}\text { CUBE } \\
\text { pour } \\
\text { un mètre } \\
\text { de } \\
\text { longueur. }\end{array}$} \\
\hline & $\begin{array}{l}\text { à } \\
\text { vive } \\
\text { arête. }\end{array}$ & $\begin{array}{c}\text { avec } \\
15 \mathrm{p} .0 / 0 \\
\text { d'aubier. }\end{array}$ & & & $\begin{array}{c}\text { à } \\
\text { vive } \\
\text { arête. }\end{array}$ & $\begin{array}{c}\text { avec } \\
15 \text { p. } 0 / 0 \\
\text { d'aubier. }\end{array}$ & \\
\hline $\begin{array}{l}\text { cent. } \\
80\end{array}$ & $\begin{array}{c}\text { cent. } \\
50\end{array}$ & $\begin{array}{c}\text { cent. } \\
58\end{array}$ & $\begin{array}{l}\text { m.c. } \\
0,550\end{array}$ & $\begin{array}{c}\text { cent. } \\
\mathbf{9 4}\end{array}$ & $\begin{array}{c}\text { cent. } \\
66\end{array}$ & $\begin{array}{c}\text { cent. } \\
78\end{array}$ & $\begin{array}{l}\text { m.c. } \\
0,608\end{array}$ \\
\hline 21 & ร0 & 58 & 0,356 & 95 & 68 & 78 & 0,608 \\
\hline 82 & 50 & 58 & $0, \overline{3} 36$ & 96 & 68 & 78 & 0,608 \\
\hline 83 & ร2 & 60 & $0, \overline{560}$ & 98 & 68 & 80 & $0,6: 0$ \\
\hline 84 & 52 & 60 & 0,360 & 98 & 70 & 80 & 0,640 \\
\hline 85 & 52 & 62 & $0, \overline{384}$ & 99 & 70 & 82 & 0,672 \\
\hline 86 & 54 & 62 & $0, \overline{584}$ & 100 & 70 & 82 & 0,672 \\
\hline 88 & 54 & 64 & 0,412 & 101 & 72 & 82 & 0,672 \\
\hline 88 & 56 & 64 & 0,412 & 102 & 72 & 84 & 0,706 \\
\hline 89 & ร้6 & 64 & 0,412 & 103 & 72 & 84 & 0,706 \\
\hline 80 & รั6 & 66 & 6,456 & 104 & 74 & 86 & 0,740 \\
\hline 81 & 58 & 66 & 0,436 & 105 & 74 & 86 & 0,740 \\
\hline 82 & ว8 & 68 & 0,462 & 106 & 74 & 86 & 0,740 \\
\hline 83 & 58 & 68 & 0,462 & 108 & 76 & 88 & 0,774 \\
\hline 84 & 60 & 68 & 0,462 & 108 & 76 & 88 & 0,774 \\
\hline 85 & 60 & 70 & 0,490 & 109 & 76 & 90 & 0,810 \\
\hline 86 & 60 & 70 & 0,490 & 110 & 78 & 90 & 0,810 \\
\hline 87 & 62 & 72 & 0,318 & 111 & 78 & 90 & 0,810 \\
\hline 88 & 62 & 72 & 0,518 & 112 & 80 & 92 & 0,846 \\
\hline 89 & 62 & 72 & 0, อ̆18 & 宜夏 3 & 80 & 92 & 0,846 \\
\hline 90 & 64 & 74 & $0, \breve{b 8}$ & 1 直 & 80 & 94 & 0,884 \\
\hline 91 & 64 & 74 & 0,548 & 115 & 82 & 94 & 0,884 \\
\hline 92 & 64 & 76 & 0,578 & 116 & 82 & 94 & 0,884 \\
\hline $\mathbf{9 3}$ & 66 & 76 & 0,578 & 118 & 82 & 96 & 0,902 \\
\hline
\end{tabular}


SIV. - Mode de classement des arbres sur pied en signaux et espèces de marine d'après leurs formes et leurs dimensions.

Pour classer, approximativement, les chênes sur pied en signaux et espèces de marine, trois choses sont à déterminer :

$1^{\circ}$ La longueur du fût ;

$2^{\circ}$ Les dimensions de l'équarrissage au milieu et au petit bout;

$3^{\circ} \mathrm{Et}$, si c'est un bois courbant, le degré de courbure du fût, ou la valeur de la flèche en millimètres par mètre de longueur.

La longueur du fût à utiliser comme bois de marine est toujours facile à obtenir au moyen d'une perche ou à l'aide d'un dendromètre.

Les dimensions de l'équarrissage au milieu et au petit bout ne peuvent pas se déterminer directement, mais on peut les déduire, avec une approximation suffisante, de la circonférence ou du diamètre de l'arbre mesuré à hauteur d'homme. L'expérience prouve, en elfet, que la circonférence ou le diamètre au milieu d'un tronc de chêne, dont la longueur ne dépasse pas celle exigée par la marine, est généralement égale aux 9/10 de la grosseur mesurée à 1 mètre ou $1^{\mathrm{m}} 30 \mathrm{du}$ sol; et, de même, que la grosseur au petit bout est égale aux $4 / 5$ de la grosseur prise à la base. On peut donc toujours, en se fondant sur ce fait d'expérience, déterminer approximativement la grosseur et, par suite, les dimensions 
d'équarrissage au milieu et au petit bout d'un arbre sur pied, sauf à modifier cette loi dans son application, si cela est reconnu nécessaire dans la forêt où on opère.

Quant au degré de courbure d'un arbre sur pied, il n'est pas toujours nécessaire de le déterminer très-exactement; car, pourvu que la courbure soit régulière, la pièce pourra toujours fournir l'un des signaux du tarif appartenant à la catégorie des courbants. Que si, cependant, on tenait à connaître, aussi sûrement que possible, le signal qu'on en pourra tirer, on déterminerait son degré de courbure, avec une approximation assez grande, en figurant la corde de l'arc avec une baguette que l'on tiendrait à la main, et en appréciant la longueur de la flèche par rapport au diamètre calculé de la pièce au milieu. Il ne resterait plus alors qu'à déterminer la valeur en millimètres de la flèche pour chaque mètre de longueur du fùt ( $\boldsymbol{P l}$. IV , fig. 1 et 3$)$.

Quand on pourra admettre la loi de décroissement des circonférences dont il a été question plus haut, on trouvera, dans l'emploi des tableaux qui vont suivre, un moyen de classer très-approximativement les arbres sur pied en signaux et espèces de marine.

Le premier de ces tableaux est applicable au classement des pièces qui doivent figurer parmi les signaux pour lesquels la marine exige l'équarrissage à vive arête (pièces de quilles, étambots, mèches de gouvernail, bittes, baux, demi-baux, barrots et étra- 
ves). Le second tableau pourra servir à classer les pièces pour lesquelles la marine accorde une tolérance de 150,0 d'aubier ou de défourni sur les dimensions de l'équarrissage. Seulement, nous devons avertir que, dans la construction de ce tarif, nous avons supposé que les arbres avaient une épaisseur d'écorce et d'aubier égale à 4 centimètres sur le rayon, ou 8 centimètres sur le diamètre. Et, comme l'épaisseur de l'aubier et de l'écorce peut varier d'une localité à une autre, ou même seulement avec l'âge des arbres, nous prévenons que, pour chaque centimètre d'aubier en plus ou en moins sur le rayon, il faudrait, pour obtenir l'équarrissage porté sur le tab̉leau, que la circonférence de base eût 8 centimètres de plus ou de moins. Par exemple, le tableau $B$ indique que pour 1 mètre 88 cent. de tour à la base, on doit avoir un équarrissage de 0 mètre $38 \mathrm{c}$. au milieu; si l'écorce et l'aubier avaient 5 centimètres d'épaisseur, il faudrait que l'arbre mesurât $1^{\mathrm{m}} 96$ de tour au lieu de $1^{\mathrm{m}} 88$ pour fournir le même équarrissage, et un $2 \mathrm{PT}$, si la pièce a le degré de courbure exigé. 
일

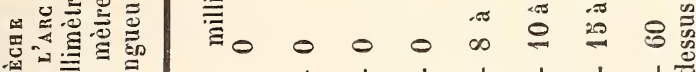

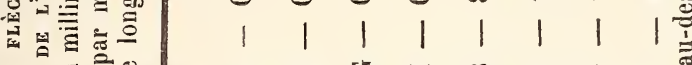

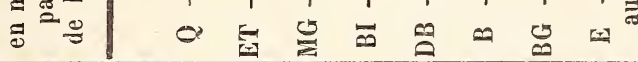

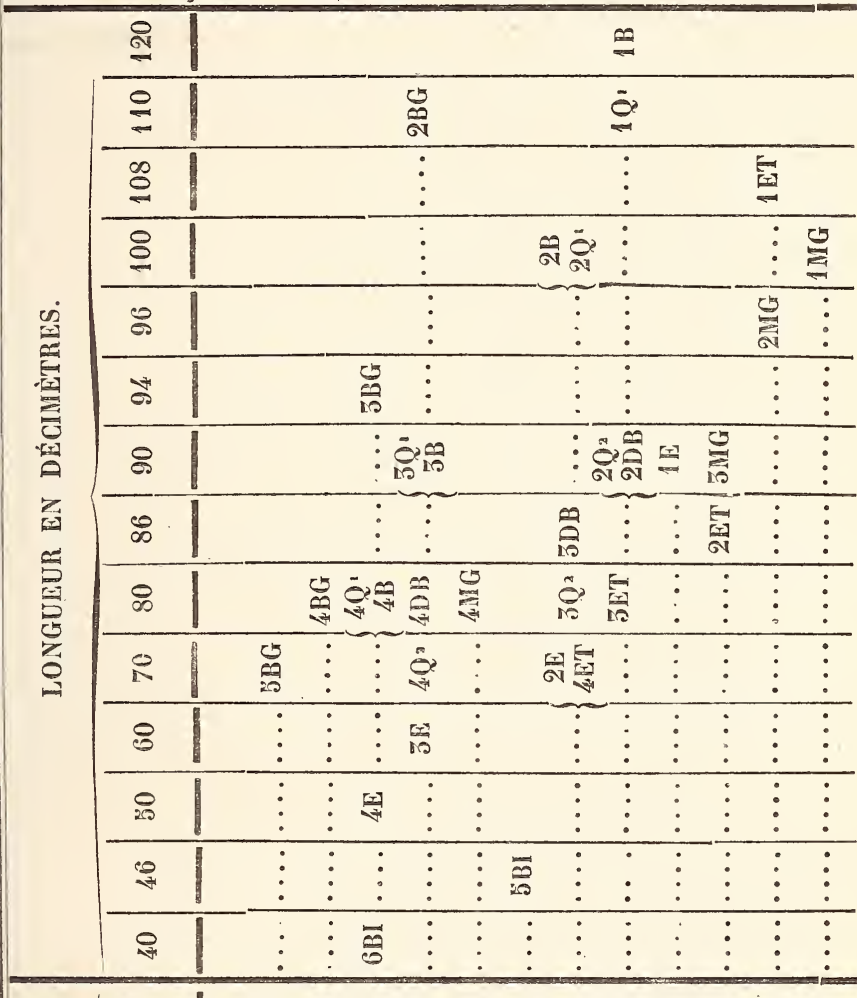

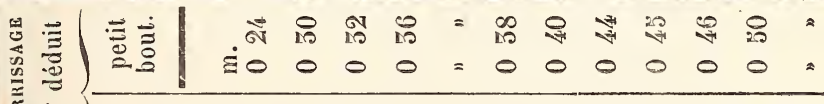

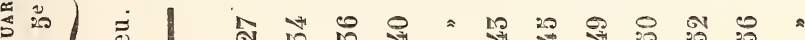
暏 -juppuodsədal $\infty$

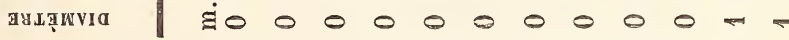

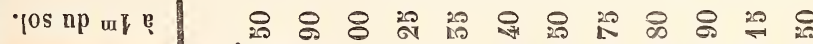

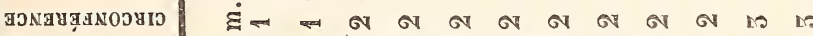


B. - Signaux qui, dans le classement, admettent à

(Tous les bois courbants, moins

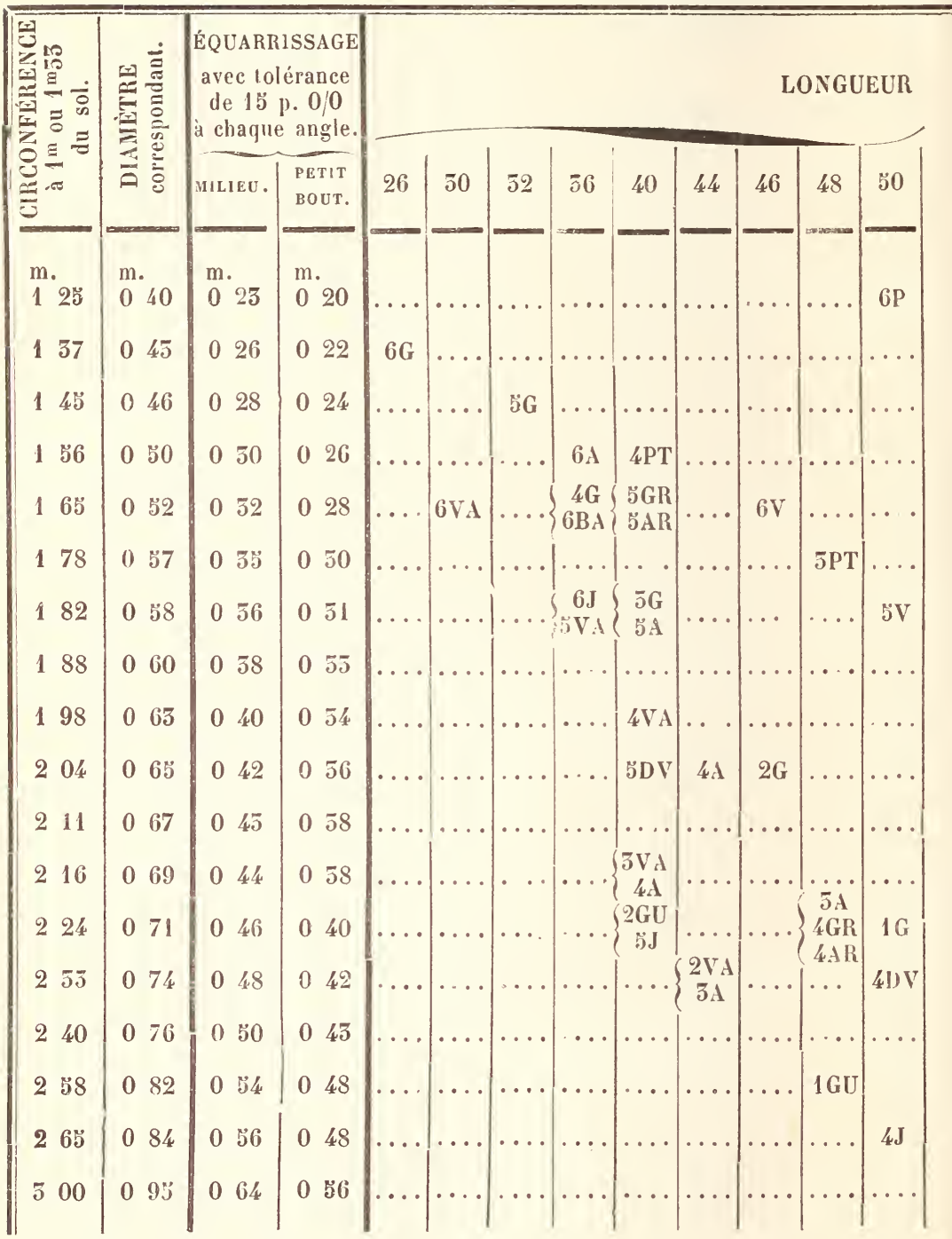

Nota. - (Voir la page 128.) 
chaque angle 15 p. 0/0 de flaches, défournis ou aubier.

l'étrave, plus le plançon.)

\section{EN DÉCIMÈTRES.}

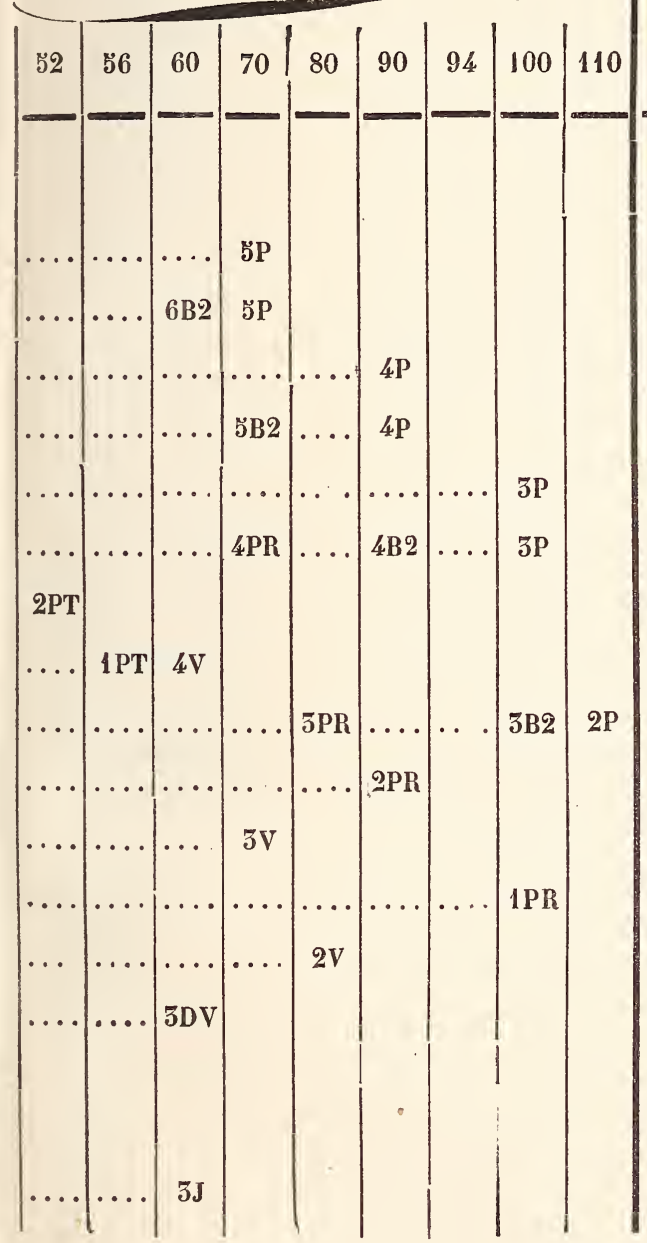

\section{FLÈCHE DE L'ARC}

en millimètres par mètre

de longueur.

millim.

$6 \mathrm{P}-0$.

$2 \mathrm{P}-0$ à 12 dans un sens;

3P courbure quelconque

$4 \mathrm{P}$ mais régulière dans

๖р l'autre.

J -25 à 35.

DV

$\underset{\mathrm{VA}}{\mathrm{BA}}\{-55$ et au-dessus.

PR - 5כ el au-dessus sur le tour, 0 à 12 sur le droit.

A -50 et au-dessus.

VA -75 et au-dessus.

PT -80 et au-dessus sur le tour, 0 à 12 sur le droit.

GU $\}-100$ et au-dlessus.

GR - 50 à 60 chaque $1 / 2$ longueur.

$\mathrm{AR}-60$ à $1201 / 2$ pied, 20 à $401 / 2$ tète.

B2 - 10 et au-dessus sur le tour, 10 à 20 sur le droit. 
NoтA. - Les indications portées dans les tableaux qui précèdent, ne peuvent être données pour certaines.

En effet, nous avons supposé, dans la construction du $1^{\mathrm{er}_{\mathrm{r}}}$ tableau, que l'équarrissage à vive arête ou sur franc bois correspondait à l'équarrissage au כ̃e déduit ou, ce qui revient au même, que le côté de l'équarrissage à vive arête est égal au cinquième de la circonférence sur écorce. Or, cela n'a pas toujours lieu, surtout dans les petits arbres dont l'aubier et l'écorce ont une épaisseur généralement plus grande, relativement au diamètre total, que dans les très-gros chênes. Il est facile de s'assurer d'ailleurs, par un calcul très-simple, que pour qu'un arbre équarri au $5^{e}$ ne renferme pas d'aubier il faut que le diamètre sur franc bois soit au moins égal aux 89 centièmes du diamètre sur écorce, ou que l'aubier et l'écorce n'aient ensemble qu'une épaisseur de $110 / 0$ du diamètre total.

De même, on a pu remarquer que la plupart des bois de marine peurent être classés par signaux et par espèces, d'après les côtés d'équarrissage mesurés au milieu et au petit bout avec une tolérance de $150 / 0$ d'aubier à chaque angle. Or, on peut s'assurer aussi que pour que l'équarrissage au כ̌ contienne $150 / 0$ d'aubier à chaque angle, il faut que le diamètre sur franc bois soit les 77 centièmes du diamètre sur écorce, ou que l'épaisseur de l'écorce et de l'aubier soit au moins égale à $230 / 0$ du diamètre total.

On voit, d'après cette observation, que l'épaisseur de l'écorce et de l'aubier, relativement au franc bois, joue un rôle important dans le classement des bois équarris et, comme cette épaisseur ne peut jamais être déterminée très-exactement tant que l'arbre est sur pied, il s'ensuit que les indications portées dans le $2^{\mathrm{e}}$ tableau ne peurent être fournies que comme des données approchées. Ajoutons seulement que, pour tous les bois auxquels la Marine accorde la tolérance de $150 / 0$ d'aubier sur l'équarrissage, ces indications sont plutôt au-dessus qu'au-dessous de la vérité. 


\section{Article II.}

Emploi des bois de chêne.

Nota. - Afin de faciliter l'étude et l'emploi des tarifs de recette de la marine, afin d'apprendre à distinguer sûrement les conditions que doit réunir une pièce de bois en grume pour être propre aux constructions navales, et la valeur particulière qu'elle peut avoir en raison de sa forme et de ses dimensions, nous allons indiquer l'emploi de chaque signal dans la construction d'un vaisseau, la place qu'il occupe dans la charpente du bâtiment et les qualités particulières que le bois doit offrir, selon l'emploi auquel il est destiné. Dans cette nouvelle nomenclature, nous suivrons à peu près l'ordre dans lequel les divers signaux sont mis en œuvre.

SI. - Emploi des pièces de quille, élambols, courbes d'étambot, étraves et brions.

Quille. - Pièce droite formant la base du bâtiment et placée dans son axe longitudinal. Elle reçoit les entailles des couples ou côtes qui composent la carcasse du navire. C'est sur elle que s'appuient, à l'avant et à l'arrière, l'étrave et l'étambot qui sont avec elle dans un même plan et dessinent les contours extrêmes du plan longitudinal. On superpose ordinairement sur la quille une contre-quille pour la renforcer, et sous la quille une autre pièce que l'on nomme fausse-quille. ( $P l . V$, fig. 1 ; q, quille; $c q$, contre-quille; $f q$, fausse-quille.)

Е́тамвот. - Pièce droite qui se dresse à l'arrière 
sur la quille de manière à former avec elle un angle plus ou moins obtus que l'on nomme quête. L'étambot porte les ferrures qui soutiennent le gouvernail. Il se relie à la quille par la courbe d'étambot, dont une branche est chevillée avec la quille et l'autre avec l'étambot. $(P l . V, f i g .1$, étambot monté sur la quille : e t, étambot; c e t, contre-étambot; fe t, faux-étambot; $d$, courbe d'étambot; $g$, chevilles.)

Étrave. - Pièce à forle courbure, formant l'avant du bâtiment; elle se relie à la quille par une pièce que l'on nomme brion. ( $P l . V$, fig. 2, étrave montée sur la quille : $e$,étrave; c e, contre-étrave; $\mathrm{tm}$, taille-mer, il forme la base de la guibre qui termine le vaisseau à l'avant.)

Brion. - Pièce courbe, droite dans sa partie inféricure pour le prolongement de la quille et formant un coude dans sa partie supérieure pour ébaucher la base de l'étrave. ( $P l . V$, fig. $2 ; b$, brion; $a$, écart ou assemblage de la quille avec le brion; $q, c q, f q$, comme dans la fig. 1.)

\section{OBSERYATIONS.}

Toutes ces pièces doivent être en bois de chêne de la meilleure qualité, parce que ce sont elles qui ont à supporter le plus de fatigue dans la marche et la manœuvre du bâtiment. Elles doivent donc être exemptes de tous défauts et notamment de ceux qui seraient de nature à diminuer la force du bois, ou à occasionner des voies d'eau. 
Le tarif de recette admet les quilles qui présenteraient un défourni (diminution sur l'équarrissage) au petit bout, pourvu que ce défourni n'existe que sur une des faces, et seulement sur une longueur égale au 1/6 de la longueur totale. Le motif de cette tolérance est que les pièces de quille doivent être entaillées sur une face pour s'assembler entre elles ou avec la courbe de brion par l'une de leurs extrémités. La même tolèrance est admise pour l'une des faces de tour de l'étrave, en raison de son mode d'assemblage avec la branche du brion. Quant à l'étambot, il est assujetti aux mêmes conditions que la quille, sauf qu'il n'est pas toléré de défourni au petit bout; cette différence s'explique par son mode d'assemblage avec la quille (Pl. V, fig. 1).

L'orme champêtre pourrait remplacer le chêne dans les pièces de quille et, en général, dans toutes les parties de la charpente qui sont sous l'eau. Mais cette précieuse essence est rare dans nos forêts et son prix est généralement supérieur à celui du chêne, bien que sa croissance soit beaucoup plus rapide (1). On en emploie cependant une quantité assez notable, dans les arsenaux de l'État, à la fabrication ou à la construction d'objets d'armeinent et, dans les chantiers du commerce, à la construction des navires de la marine marchande, des bateaux de pêche, etc. Ces bois provienuent ordinairement d'arbres plantés isolément dans des propriétés particulières, et ne présentent que rarement les dimensions et les qualités nécessaires aux grands emplois.

Les pièces de quille, étambots, étraves et brions sont rares dans nos forêts, du moins en première espèce.

(1) Le cahier des charges de 1857 autorise les fournisseurs de la marine à remplacer le chène par l'orme champêtre dans la fourniture des pièces de quille de $1^{\mathrm{re}}$ et $2^{\mathrm{e}}$ espèces. On cite particulièrement en France le département du Nord, comme produisant des ormes propres aux grands emplois de la marine. 


\section{S II. - Emploi des varangues, genoux et allonges.}

La membrure d'un vaisseau, autrement dit les couples, se composent de différentes pièces énumérées ci-dessous et qui entrent dans leur assemblage suivant les formes qu'elles affectent. Ces formes leur sont imposées par le tracé du bâtiment à construire. En commençant par le bas, ce sont :

Les varangues plates. - Pièces à une courbure qui s'entaillent par le milieu, se placent à cheval sur la quille, perpendiculairement à celle-ci, et s'étendent des deux côtés sur une certaine longueur, avec leur convexité par le bas. Ces pièces s'emploient dans la partie centrale.

Les demi-varangues. - Pièces à une courbure accolées ou juxta-posées aux précédentes pour former le couple, lequel se compose, comme son nom l'indique, de deux plans de bois. Les demi-varangues se joignent bout à bout. - Les pièces qui forment les deux plans de chaque couples sont assujetties par des chevilles carrées en fer appelées gougeons. $(\boldsymbol{P l}$. V, fig. 3, partie basse d'un couple du milieu d'un vaisseau à trois ponts : $v p$, varangue plate; $d v$, demi-varangue placée devant ou derrière la varangue plate, dans le second plan de bois du couple; $q$, quille; $c$, carlingue, pièce de bois placée parallèlement à la quille, à l'intérieur du bâtiment; ff, 
chevilles reliant la carlingue et la quille; $k$, gougeon.)

Les Genoux. - Ce sont des pièces à une courbure, placées à la suite des varangues et des demi-varangues dans les parties cintrées du couple. ( $P l . V$, fig. $3, g$, genou.)

Les allonges et les bouts D'Allonge. - Pièces à une courbure servant à compléter la formation du couple jusqu'à la partie supérieure du bâtiment. Cés pièces sont apposées bout à bout, les unes à la suite des autres, de manière que les joints ou écarts des pièces d'un plan correspondent à peu près au milieu des pièces de l'autre plan. ( $P l$. VI, fig. 1. a, allonge; $b a$, bout d'allonge.)

Les varangues acculÉes. - Ces pièces à une forte courbure s'emploient comme les varangues plates dans les parties moins plates de la cale des bâtiments. (Pl. V, fig. 4, partie basse d'un couple de vaisseau vers les extrémités : $v a$, varangue acculée; g, genou; c, carlingue; q, quille.)

Les Genoux DE REvers. - Ce soni des pièces à deux courbures très-prononcées qui remplacent les varangues dans les parties aiguës de l'avant et de l'arrière. $(P l . V I, f g .1$, coupe d'un vaisseau dans les parties fines et acculées : $g r$, genou de revers; c, carlingue; $q$, quille.) 
Les allonges De revers. - Pièces à deux courbures qui remplacent les genoux dans les parties aiguës de l'avant et de l'arrière. ( $P l$. VI, fig. 1, $a r$, allonge de revers; $a$, allonge; $b$ a, bout d' allonge.)

En résumé, on voit que, perpendiculairement au plan qui passe par l'axe de la quille; s'élèvent une suite de fermes doubles, composées chacune de deux rang de pièces de bois appliquées les unès à côté des autres et chevillées solidemeñt ensemble. Cé sont lés couples dont l'ensemble forme la membrure du bâtiment. Entre deux couples, on laisse toujours un intervalle de quelques centimètres qu'on nomme maille. Ces vides rendent la charpente plus légère et facilitent la circulation de l'air et l'écoulement de l'eau entre les différentes pièccs quiue des contacts trop multipliés feraient pourrir très-rapidement.

\section{OBSERVATIONS.}

Les pièces qui servent à former la membrure ou les couples des bâtiments de la marine militaire sont en bois de chêne. Ce que l'on demande spécialement aux bois destinés à cet emploi, c'est d'être sains, nerveux, exempts de défauts qui pourraient engendrer ou favoriser la pourriture et, par suite, compromettre la solidité et la durée du bâtiment. On conçoit en effet que les pièces les plus importantes, celles qui forment la partie inférieure des couples, sont d'autant plus exposées à s'échauffer et à pourrir qu'elles sont placées dans un milieu móns aéré, plús humide et plus chaud. Mais les fortes gercures; les fibres 
torses (1), de légères roulures, les nœuds sains et les cavités qui proviennent du sondage des pièces, ne s'opposent pas à leur emploi dans cette partie de la charpente. Les fortes gerçures et les fentes sont, au contraire, un signe qui atteste la qualité de ces bois comme force et durée; seulement, pour prévenir les voies d'eau qu'elles pourraient occasionner, on prend la précaution de les calfater après que les pièces sont mises en œuvre.

Le tarif de recette ne dit pas que ces pièces puissent êlre admises avec un défourni, mais on lit dans le cahier des charges de 1857, article 24: a Sauf les exceptions portées au tarif pour les pièces de quille, étambots, mèches de gouvernail, bittes, baux, barrots de gaillard et étraves, l'aubier et les défournis ou flaches existant aux angles des pièces ne donneront lieu à aucune réduction, lorsqu'ils n'excèderont pas, à chaque angle, quinze pour cent de la largeur de la pièce à l'endroit correspondant à l'aubier ou au défourni, et lorsque d'ailleurs les faces seront saines et sans défectuosités. - Le défourni ou l'aubier se mesurera sur les faces des pièces et non diagonalemeint. » Cette tolérance est extrêmement importante et nous la signalons comme facilitant beaucoup la fourniture des bois courbants les plus rares par leurs formes et leurs dimensions.

Parmi les bois qui entrent dans la construction de la membrure, se trouvent des pièces à très-forte courbure que l'on ne rencontre que rarement dans nos forêts, du moins en $4^{\text {re }}$ et $2^{\circ}$ espèces, parce que dans les exploitations nous arons une tendance générale à ne réserver que des arbreミ droits et d'un beau port, comme étant moins dummageables aux sous-bois par leur couvert Il serait cependant d'un grand intérêt pour l'Etat d'élever, dans les futaies comme dans les taillis, des chênes propres à fournir des courbes et des courbants à la marine, en réservant spécialement ceux qui, placés sur la limite des forêts, sur les lisières des coupes, ou sur le bord des chemins, affectent les formes les plus rares et les plus recherchées.

(1) Voir au chapitre suivant les défauts et qualités des bois. 
Les varangues acculées, les genoux et les guirlandes qui arrivent dans nos ports proviennent, pour la plupart, de chênes qui ont crû isolément. On voit beaucoup de ces chênes plantés en haie ou en bordure le long des fossés qui servent de limites aux propriétés, dans les départements de l'ouest de la France. De là le nom de bois de fossé ou bois champêtre que l'on donne, dans les ports, aux pièces de marine qui proviennent de ces arbres. Cos chênes sunt souvent viciés pas suite des élagages répétés auxquels ils ont été soumis; mais, quand ils sont exempts de vices, leur bois est réputé de la meilleure qualité et valoir celui des chênes d'Italie. Tels sont ceux qui proviennent du Calvados ou des contrées voisines, et ceux qui viennent des Landes et que l'on désigne plus spécialement sous le nom de bois de Bayonne (1).

S IIl. - Emploi des baux, courbes de pont, guirlandes et courbes de jollereau.

Pour compléter la construction du bâtiment comme coque, on applique à l'intérieur et à l'extérieur des couples un revêtement de pièces de bois, juxta-posées longitudinalement, que l'on nomme bordages et qui, avec les couples, forment la muraille du bâtiment. Ce revêtement prend à l'intérieur le nom de vaigrage, et les bordages sont alors des vaigres. Les

(1) J'ai vu de ces chènes à Cherbourg et à Brest et j'ai élé frappé de leur qualité, comme force, et de la rapidité avec laquelle ils ont crû. Leur bois, dur comme de la corne, se prêterait mal à des ouvrages de fente; sa couleür est d'un blanc jaune rosé, et les couches concentriques annuelles alteignent souvent plus d'un centimètre d'épaisseur. Du reste, ces bois sont généralement courts, noueux, mal conformés et fortement gercés; ils seraient d'un emploi difficile dans les constructions ordinaires et d'un mauvais usage comme sciages. 
vaigres ordinaires se nomment vaigres de point; les vaigres de plus forte épaisseur que l'on place dans les parties inférieures pour renforcer les fonds, se nomment vaigres d'empâture. (Pl. $V$; fig. 3, v, vaigres de poirt; $v$ e, vaigres d'empâture.)

Le revêtement extérieur du vaisseau, ou la pose des bordages, n'a lieu qu'après le vraigrage ou le revêtement intérieur et l'établissement de la charpente des ponts. Cette charpente doit être très-solide, parce qu'elle sert à relier entre elles les membrures opposées de chaque bord, et parce que, dans les vaisseaux de guerre, les ponts sont surtout destinés à porter l'artillerie. Voici les principales pièces qui entrent dans cette construction.

Les BAUx. - Ce sont des pièces à courbure légère que l'on place à l'intérieur du bâtiment, dans un sens perpendiculaire à la quille, pour recevoir les bordages ou planchers des ponts. Les baux s'appuient par leurs extrémités sur une sorte de corniche en bois que l'on nomme bauquière. Cette corniche est formée de forts bordages placés longitudinalement les uns à la suite des autres contre la membrure, et solidement chevillés avec elle. Les baux ont leur faxe convexe tournée vers le ciel, de manière à déterminer la forme en dos d'âne qu'il convient de donner à la surface du plancher des ponts, pour faciliter l'écoulement des eaux.

Dans les vaisseaux de guerre, on est souvent obligé de faire les baux de deux ou de trois pièces, à cause 
de la grande disfance qu'il y a d'un bord à l'autre. De là, les demi-baux qui arc-boutent l'un contre l'autre et sont assemblés et fortement reliés entre eux par des chevilles rivées ou boulonnées.

Les baux qui sont destinés au pont supérieur ou pont des gaillards, prennent le nom de barrots de gaillard.

Les courbis De Pont. - Pièces courbes que l'on place aux angles des ponts avec les murailles; elles sont formées par l'insertion d'une branche dans le tronc de l'arbre. Ces courbes ont chacune une branche chevillée avec un des baux et l'autre avec la membrure. Elles ont pour objet de maintenir l'union des ponts avec les parties latérales de la charpente. $(P l . V I, f i g .2$, section d'un vaisseau à trois ponts, perpendiculaire à la quille : $C V$, couple du vaisseau; $b$, bordages; $v$, vaigres; $v$ e, vaigre d'empâture; $p b$, plat bord; $B G$, barrot de gaillard; $\boldsymbol{B} \boldsymbol{P}$, bau de pont ; $\boldsymbol{D} \boldsymbol{B}$, demi-bau; $b p$, bordages des ponts; $f g$, fourrure de gouttière, dans l'angle du pont avec la muraille; elle est percée d'ouvertures ou dalots servant à l'écoulement des eaux; $c p$, courbe de pont; c p f, courbe de pont en fer; $h r$, hiloires renversées, placées dans toute la longueur du vaisseau pour relier les baux entre eux; $e p c$, épontille de cale servant à soutenir les nonts; $e p$, épontille de pont; e $m$, emplanture du mât; $m$, mât ; $b i$, bitte de pied de mât.)

Les guirlandes. - Pièces à forte courbure que 
l'on emploie à l'irtérieur du bâtiment, à l'avant et à l'arrière, pour relier et solidifier les pièces de la membrure et empêcher l'écartement des bords.

La courbe De jottereau. - Pièce courbe, destinée à soutenir l'éperon saillant que porte l'avant du navire. L'une des branches du jottereau s'applique sur la membrure et l'autre sur cet éperon.

OBSERYATIONS.

Les pièces que l'on emploie pour former la charpente des ponts sont: les baux, les demi-baux, les barrots de gaillard et lès courbes de pont. Ces mêmes pièces servent aussi avec les güirlandes à relier et à solidifier les parties opposées des couples et à empêcher l'écartement des bords.

Les baux et les demi-baux entrent en très-grand nombre dans la construction de la charpente des ponts. On ne compte pas moins de 150 baux dans la charpente d'un vaisseau de premier rang. Le Montelello, vaisseau de 120, dont le modèle est à l'École forestière, en porte 148. - Les baux et les demi-baux se font en bois de chêne, ou en bois de pin sylvestre du Nord, de pin Laricio et de pin des Florides. Mais la plus grande partie dés baux sont en bois résineux, et l'on n'emploie le chêne à cet usage que dans les ponts inférieurs et dans les parties de la chärpente des autres ponts qui supportent le plus de fatigue, ou qui sont le plus exposées aux causés de destruction. La raison de la préférence que l'on dỏnne au pin sur le chêne dans cet emploi, c'est que le pin étant plus léger que le chêne charge moins le bâtiment, et que d'ailleurs il dure et résiste au moins autant que le chêne lorsqu'il est mis en œuvre dans de bonnes conditions. On emploie aussi le pin en demi-bau, mais plus rarement que le chêne, parce que les chevilles qui servent à relier 
les deux parties d'un bau tiennent moins bien dans le bois résineux.

Les baux de chêne de $1^{\text {re }}$ et $2^{\mathrm{e}}$ espèces sont assez rares dans nos forêts, parce que ce sont des pièces de grande longueur, d'un fort équarrissage et dont la courbure doit être très-régulière. Ceux de pin sylvestre étant souınis aux mêmes conditions, quant aux dimensions, à la forme et à la qualité des pièces, seraient aussi très-difficiles à trouver, surtout à cause des dimensions de l'équarrissage ; mais, jusqu'à présent, on n'en a point cherché dans les pineraies de France, sauf en Corse où les forêts de Laricio fournissaient naguère, sous ce rapport, de précieuses ressources à la marine.

Les baux étant des pièces de longueur, pour lesquelles on n'admet ni défourni, ni tolérance d'aucune sorte en ce qui concerne la qualité du bois, ne pourraient être fournis en grande quantité que par des arbres élevés en massif de futaie. Mais les bois qui viennent en futaie pleine n'atteignent pas toujours, à l'âge marqué par la révolution, les dimensions nécessaires pour suffire aux conditions d'équarrissage des pièces de marine de $\mathbf{1}^{\text {re }}$ et même de $2^{\circ}$ espèce. Tout au plus trouve-t-on aujourd'hui, dans les massifs de futaie de chêne ou de pin, des arbres qui, à l'âge d'exploitation, ont les dimensions nécessaires pour donner des barrots de gaillard. - Pour obtenir des arbres qui offrissent ces grandes dimensions à l'époque de leur exploitation, il faudrait que les futaies de chêne et de pin sylvestre fussent éclaircies beaucoup plus fortement qu'on ne le fait d'habitude, à partir du moment où les arbres ont atteint toute leur croissance en hauteur de fût (1); ou bien il faudrait, qu'au moment des colipes de régénération, on fît une réserve assez importante des arbres qui, par leurs formes et leur état de végétation, pourraient fournir, au bout d'un temps plus ou moins long, des

(1) Voir le Cours de culture des bois de MM. Lorentz et Parade. Livre $3^{\mathrm{e}}$, chapitre $2^{\mathrm{e}}$, article III et XIV. 
pièces de $1^{\text {re }}$ et $2^{\mathrm{e}}$ espèces aus constructions navales (1). Sans cela, les baux de chêne, de même que les quilles et les autres bois droits de $1^{\text {re }}$ et $2^{\mathrm{e}}$ espèces, seront toujours fournis en grande majorité par des arbres élevés sur taillis, lesquels ne peuvent jamais donner, à nombre égal, la même quantité (parce qu’ils sont souvent viciés ou mal conformés) de bois droits propres aux grands emplois, que des arbres de mêmes dimensions venus en futaie (2).

Les courbes de pont sont en bois de chêne; ces pièces sont assez difficiles à trouver, du moins en quantité suffisante pour les besoins de la marine, aussi les remplace-t-on généralement aujourd'hui par des courbes en fer. Ces courbes ont aussi l'avantage d'occuper moins de place dans l'intérieur du bâtiment que les courbes de pont en bois; par contre, elles n'ont pas autant d'élasticité.

Le plancher des ponts se fait en bois de chêne et de pin. Les planches qui servent à cet usage reçoivent le nom de bordages de pont. Leur largeur varie de quinze à vingt centimètres, leur épaisseur de quatre à douze centimètres. Les ponts et les faux ponts sont tout entiers bordés avec du pin, à l'exception de la partie voisine des bords, sur laquelle roulent les canons dans la manœuvre, qui est bordée avec du chêne. On évite aussi d'employer du bois susceptible d'un très-beau poli, le pin des Florides par exemple, pour border le pont des gaillards, parce qu'il devient trop glissant.

(1) Voir le Cours de culture des bois de MM. Lorentz el Parade, Livre $3^{e}$, chapitre $2^{\mathrm{e}}$, article III.

(2) Dans la construction de la nouvelle flolte blindée, la Marine a généralisé l'emploi du fer, et les baux sont en fers laminés à double T. 
§IV. - Emploi des plançns, pièces de tour, préceintes et bois à deux bouges.

Plançons. - C'est dans le but de se procurer des bordages de toutes dimensions que la marine recherche les plançons, pièces droites ou ayant peu de courbure que l'on débite à la scie dans les arsenaux.

PiÈces de tour. - Ce sont des pièces à une courbure, destinées à être employées comme bordages à l'avant et à l'arrière, et à former les joues et les hanches du bâtiment. Les pièces de tour sont aussi employées comme les guirlandes.

Précerntes. - Ce sont des bordages de plus fortes dimensions que le reste du revêtement, que l'on place à la hauteur des ponts et qui doivent recevoir les ferrures qui servent à la manœuvre des canons. (Pl. VI, fig. 2.p, préceintes; $b$, bordages; v, vaigres.)

Les préceintes de tour sont celles que l'on emploie à l'avant et à l'arrière; elles ont une courbure plus forte que celles qui s'appliquent dans la partie centrale de la muraille.

Bors a deux bouges. - Ce sont des pièces à deux courbures dans deux plans différents, que l'on emploie comme les préceintes, ou dont on se sert pour dessiner la forme extérieure de l'arrière du vaisseau.

$(P l . V I l, f g .1$, coupe longitudinale de la muraille d'un vaisseau faisant voir le boisage : $c$, cou- 
ples; $P R$, préceintes; $P$ B. plat bord; $S 1$, sabords de la $1^{\text {ro }}$ batterie; $S 2$, sabords de la $2^{\mathrm{e}}$ batterie; $S 3$, sabords de la $3^{\mathrm{e}}$ batterie; $S$ G, sabords des gaillards; $m$, maille ou écart de deux couples.)

\section{OBSERYATIONS.}

Les plançons, les pièces de tour, les préceintes de tour et les bois à deux bouges sont des signaux qui servent au bordage intérieur et extérieur du bâtiment. Ces pièces s'emploient entières, ou refendues à la scie en madriers plus ou moins épais, selon la place qu'ils doivent occuper. Les bordages les plus épais, après les préceintes, sont ceux qui occupent la partie la plus évasée de la coque; les autres vont en diminuant d'épaisseur en se rapprochant de la quille ou du plat bord.

La plus grande partie des bordages sont en bois de chêne; les uns proviennent du débit des pièces que nous venons d'énumérer, les autres nous arrivent tout façonnés des provinces de la Baltique, et sont soumis atix conditions de recette indiquées dans le tarif ci-après.

Tarif spécial de recette des bordages de chêne de la Ballique.

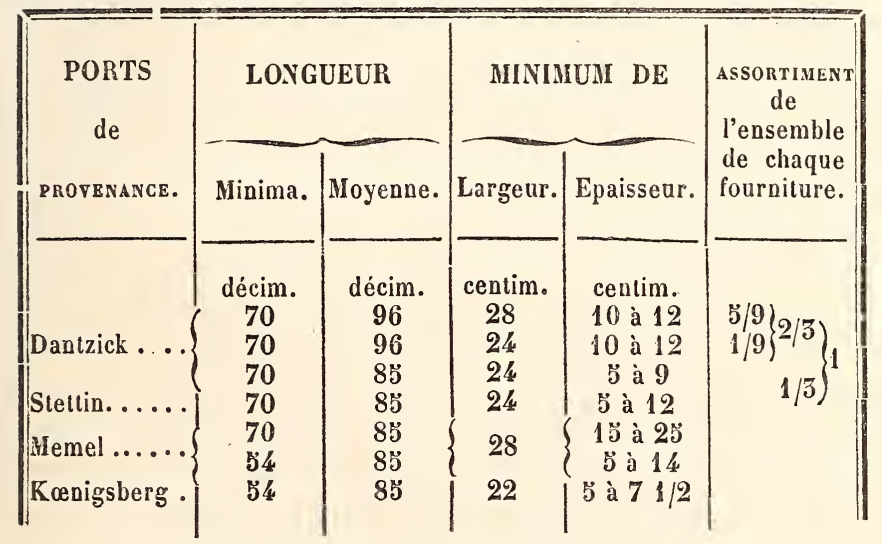


Le pin sert aussi quelquefois à cet usage, mais seulement dans les parties voisines du plat bord. On prétend même que le pin sylvestre ou du Nord de bonne qualité, le pin des Florides et le mélèze pourraient être employés comme bordages dans les parties basses de la coque, sous l'eau, et qu'ils feraient un aussi bon usage que le chêne. Mais ces bois deviennent trop rares pour recevoir cet emploi en grand. Il serait done d'un grand intérêt pour notre pays d'arriver à produire en France du pin sylvestre qui pût remplacer celui que l'on fait venir des provinces de la Baltique, pour les besoins de la marine impériale. Nous avons vu une quantité considérable de bordages de pin dans les magasins de Cherbourg, et nous pouvons affirmer qu'ils ne sont pas supérieurs en qualité à ceux que l'on pourrait tirer des forêts de Bitche et de Haguenau. Mais nous reconnaissons que ces forêts, dans l'état actuel de leurs peuplements, ne pourraient fournir que des quantités peu importantes de ces bois, parce que la largeur des bordages de pin exige que les arbres dont ils proviennent aient des dimensions en diamètre, défalcation faite de l'aubier, que l'on ne rencontre pas communément dans les conditions actuelles d'exploitation des pineraies de ces contrées. On en jugera du reste par l'extrait que nous donnons ci-après du tarif général de recette des bois de marine. 
Tarif spécial de recelte des bois résineux de la Baltique.

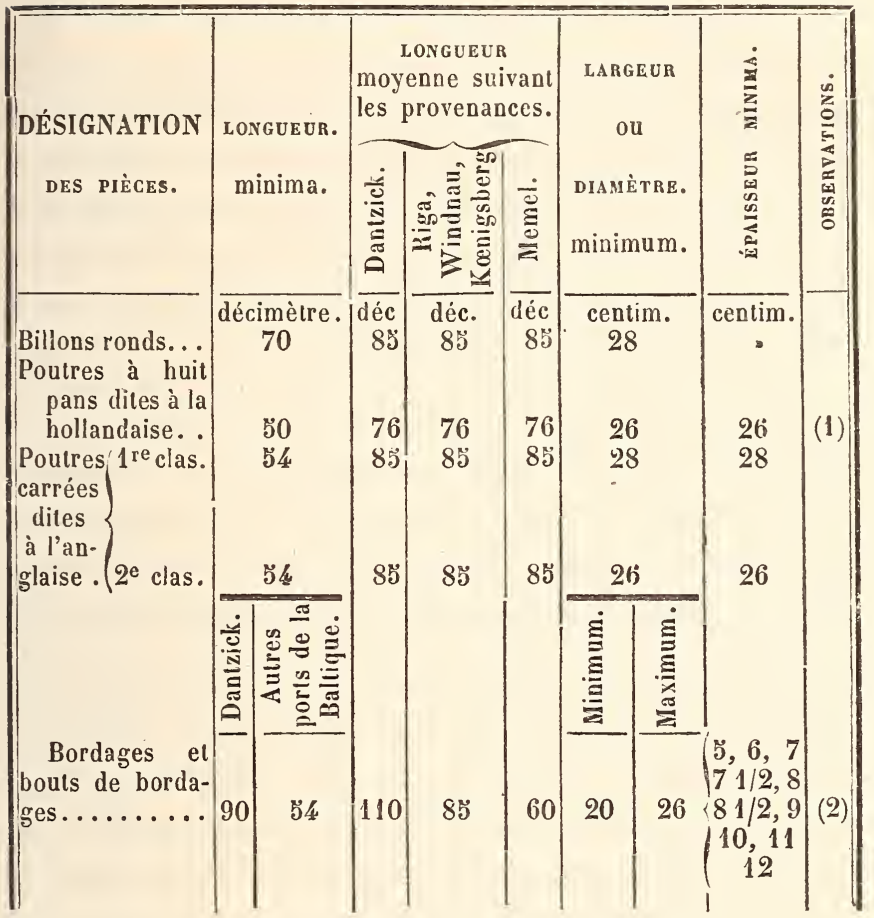

Les plançons, les pièces de tour, les préceintes de tour, les bois à deux bouges doivent être exempts de tous défauts, tels que la roulure, la gélivure, la torsion des fibres (3), etc., qui

(1) Les tolérances à l'égard de l'aubier, des nœuds et des fentes seront indiquées dans le cahier des charges.

(2) Les cahiers des charges fixeront les proportions suivant lesquelles les bordages de chaque épaisseur devront entrer dans les fournitures : ne pourront ètre classés comme bordages ceux où le cœur serait compris entre les deux faces, ou marqué sur les deux faces, ou même marqué sur une seule face, s'il pénètre jusqu'au milieu de l'épaisseur.

(3) Voir an chapitre suivant les défauts et qualités des bois. 
ne permettraient pas le débit en bordages. On conçuit, en effet, que les bordages qui proviendraient du débit d'un bois tors ou virant n'auraient pas toute la solidité désirable, parce que, dans le sciage des pièces, les fibres seraient coupées à plusieurs endroits et perdraient beaucoup de leur force. Mais, pour les pièces de chêne que l'on doit employer comme bordages, on n'exige pas que le bois soit aussi dur, aussi nerveux que pour les autres parties de la membrure, parce que les bois très-nerveux sont les plus disposés à se fendre, et que les grosses fentes pourraient occasionuer des voies d'eau.

Le hêtre peut aussi servir au bordage extérieur des bâtiments, mais seulement dans la partie de la coque qui est sous l'eau. Le vaisseau le Conquérant qui a été construit sous le premier Empire et qui a été démoli depuis peu d'années, était bordé avec du bois de hêtre qui a fait un très-bon usage. Le défaut que l'on reproche surtout au hêtre, c'est qu'il se voile beaucoup quand il est mis à sec, et que son bois se pique et s'altère promptement quand il est exposé aux alternatives de sécheresse et d'humidité. On avait espéré pouvoir remédier à cet inconvénient en employant du hêtre injecté et quelques hâtiments de transport ont été bordés d'un côté en chêne et de l'autre en hêtre injecté. Mais il a été reconnu que le sulfate de cuivre ne se fixe pas dans le hois à l'état de combinaison; l'eau de mer, qui filtre toujours en plus ou moins grande quantité par les murailles du bâtiment, l'entraîne avec elle dans le fond de la cale, et y produit une atmosphère toxique, dangereuse pour la santé de l'équipage.

$\S \mathrm{V}$. - Emploi des biltes, mèches de gouvernail, jas d'ancre.

Les BITtEs sont des pièces droites qui sont fixées debout à peu de distance des mâts. Ces pièces sont garnies de trous, de ferrures et de rouets pour la 
manœuvre des mâts supérieurs et des vergues. (Pl. VI, fig. 2. b i, bitte.)

La mèche de gouvernall. - Pièce principale qui reçoit le trou dans lequel passe la barre destinée à manouvrer le gouvernail. $(P l$. VII, fig. 2, gouveruail de vaisseau : $M G$, mèche de gouvernail; $a$, trou de la barre; $b$, entailles pour les ferrures destinées à porter le gouvernail.)

Le JAS D'ANCRE. - Pièce à faible courbure embrassant la verge de l'ancre au-dessous de l'organeau. Dans l'emploi, le jas d'ancre se compose de deux pièces de bois juxla-posées perpendiculairement au plan des becs, et destinées à empêcher l'ancre de tomber à plat au fond de la mer quand on mouille.

Les POUTRES, solives ET ACCOREs sont des signaux de déchéance provenant de bois viciés ou fendus. - Les accores en particulier servent à soutenir le bâtiment sur cale pendant sa construction.

OBSERVATIONS.

De toutes les pièces de bois qui sont employées dans les constructions navales, la mèche de gouvernail est celle qui présente le plus fort équarrissage. De plus la pièce doit être tout à fait droite et sans défourni, le bois sain, nerveux, exempt de tout défaut et particulièrement de la torsion des fibres. C'est dire combien ces pièces doivent se rencontrer rarement dans nos forêts; cependant, on ne voit pas dans le cahier des charges relatives aux fournitures de la marine que ce signal se paie 
arec prime, comme les quilles et les étambots de $1^{\text {re }}$ espèce, ou comme les courbes de $1^{\mathrm{re}}$ et $2^{\mathrm{e}}$ espèces. Cela tient peut-être à ce que la mèche de gouvernail peut avoir une tout autre forme que celle qu'on lui voit dans la figure 2, Pl. VII, surtout depuis la modification que l'on a dû apporter à la construction de l'arrière dans les bâtiments à hélice, et à l'usage qui s'est introduit depuis peu de substituer le fer au bois dans la partie supérieure de cette pièce.

Article III.

Bois résineux.

§I. - Des résineux qui servent aux constructions navales.

La marine emploie dans ses constructions une quantité considérable de bois résineux parmi lesquels figurent au premier rang les pins sylvestre, laricio et des Florides.

Le sapin et l'épicéa ne sont employés dans la marine militaire qu'à des ouvrages de menuiserie. Ces bois proviennent, pour la plus grande partie, des provinces de la Baltique (Russie, Suède et Norwége), et sont fournis à nos ports sous la forme de billons ronds, de poutres équarries à quatre ou à huit pans et de planches de diverses dimensions.

Le mélèze est peu employé parce qu'il est rare, et qu'il est surtout assez difficile de s'en procurer qui ait des dimensions convenables. Les bois de cette essence qui arrivent dans nos ports provieńnent de l'Adriatique et sont connus sous le nom de mélèzes de Trieste. Le mélèze peut servir à des 
emplois importants tels que baux, barrots de gaillard et bordages, quand il a des dimensions suffisantes et qu'il est de bonne qualité.

Le pin sert à faire des baux, des demi-baux et des barrots de gaillard qui sont assujettis aux mêmes conditions de recelte que les baux et barrots de chêne. Il esí aussi très-recherché pour bordages, mais son emploi spécial et essentiel c'est la mâture. Le pin sert exclusivement à la màture des bâtiments de la marine militaire, parce que de tous les bois résineux le pin est le plus résistant (1), le plus élastique et le plus durable. On le préfère au sapin, dont on fait usage cependant dans la marine du commerce, parce que le bois de sapin ne renfermant pas de résine se dessèche promptement et profondément, qu'il est sujet à s'échauffer, à se pourrir et dure peu, parce qu'il est moins élastique et que les mâts de cette essence ne se redressent pas exactement, comme ceux de pin, quand ils ont été courbés sous l'action d'une pression forte et quelque

(1) Ce qui fait la véritable supériorité du pin sur le sapin, c'est la résine concrète qui imprègne ses tissus et le garantit contre toutes les causes de destruction dont sont plus particutièrement menacés les bois prompts à se dessécher. Quant aux autres qualités, des expériences faites en 1846, par une commission composée de forestiers et d'ingénieurs civils, militaires et maritimes, ont consiaté que les sapins des forêts du département de l'Aude, arrondissement de Limoux, offrent une plus grande force de résistance ou de tenacité que les autres bois résineux (le pin des Florides excepté), tels que le pin du Nord, le pin du Canada, le mélèze de Trieste, l'épicéa el le sapin de Trieste que l'on emploie dans les chantiers de la marine à Toulon. 
peu prolongée. Mais les pins que nous produisons en France n'ont ni les dimensions, ni les formes, ni les qualités de bois réclamés pour la mâture, et jusqu'à présent on a préféré pour cet usage le pin sylvestre du nord de l'Europe, le pin des Florides (pinus australis), et le pin laricio ou de Corse.

Le pin laricio peuple des étendues considérables en Corse et ses excelientes qualités sont bien connues de nos constructeurs. A son sujet, on lit ce qui suit, dans un mémoire adressé à M. le Ministre de la Marine par un ingénieur qui fut chargé, vers 1845 , de parcourir les forêts de la Corse et de faire connaître approximativement les ressources qu'elles peuvent offrir aux constructions navales : "Le pin D laricio croît dans des sols granitiques très-peu "profonds, son grain est fin et serré, ses couches 》 annuelles sont étroites, ce qui est le caractère (1) ") des bois de bonne qualité; son tronc très-droit " parvient à une très-graude élévation sans branches 》) et semble destiné par son port à la mâture; la „ résine y est abondante, ce qui lui assure de la ఐ flexibilité et de la durée. Son aubier varie de 20 à 30 centimètres sur le diamètre et, dans certaines " localités, il n'est que de 8 à 10 cent. " - L'auteur de ce mémoire ajoute que, dès 1787 , on tira de la

(1) Un bois résineux dont le grain est fin et serré est généralement de bonne qualité, mais on n'en peut pas dire autant de tous les bois dont les couches annuelles sont étroites, car pour le chène notamment, plus son accroissement est rapide, toutes circonstances égales d'ailleurs, plus son bois a de qualité. 
Corse un grand nombre de mâts des plus fortes dimensions, et que pendant 10 ans, de 1812 à 1822, les pins de Corse suffirent à l'alimentation du port de Toulon. Enfin, dans ces dernières années, on a constaté que ces forêts renfermaient encore un bon nombre d'arbres propres à donner des mâts, des mâtereaux, des plançons, des baux et des espares. La difficulté est de sortir ces bois des forêts qu'ils peuplent et de les amener jusque dans les ports d'embarquement de la Corse. Or, en tenant compte des dépenses que coûterait l'établissement des routes nécessaires à la vidange de ces produits, et en affectant cette dépense uniquement $a ̉$ la production forestière actuelle, on a calculé que ces bois rendus à Toulon coûteraient à l'Etat moins cher que ceux qu'il fait venir du nord de l'Europe et des Florides. On va même jusqu'à affirmer que si ces forêts étaient convenablement traitées et aménagées, leurs produits suffiraient aux approvisionnements de nos arsenaux en bois résineux, et notamment en mâtures: qu'il est si difficile de se procurer à présent, et dont la rareté augmente tous les jours.

En présence de ces faits, nous croyons utile de faire connaître les conditions générales que doivent présenter les bois résineux dont on se sert pour la mâture dans les constructions navales. 
S II. - Proportions el classement de la mâture par degré de qualité el d'utilité.

\begin{tabular}{|c|c|c|c|c|c|c|c|}
\hline DÉSIGNATION. & 离 & 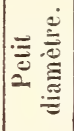 & 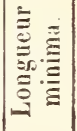 & DÉSIGNATION. & 可 & 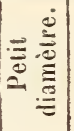 & 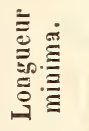 \\
\hline $\begin{array}{c}\mathrm{Il}^{\mathrm{e}} \text { et IIIe } \\
\text { classes. } \\
\text { Mèches et } \\
\text { jumelles supé- } \\
\text { rieures et infé- } \\
\text { rieures (1). }\end{array}$ & \begin{tabular}{|c|} 
cent \\
78 \\
75 \\
72 \\
69 \\
66 \\
63 \\
60 \\
57 \\
54 \\
51
\end{tabular} & $\begin{array}{c}\text { cent. } \\
52 \\
50 \\
48 \\
46 \\
44 \\
42 \\
40 \\
38 \\
36 \\
34\end{array}$ & $\begin{array}{l}28 \\
27 \\
26 \\
25 \\
24 \\
25 \\
22 \\
21 \\
20 \\
19 \\
18 \\
17\end{array}$ & $\begin{array}{l}\text { Màls } \\
\text { tronçonnés. }\end{array}$ & \begin{tabular}{|l} 
cent. \\
81 \\
78 \\
75 \\
72 \\
69 \\
66 \\
63 \\
60 \\
57 \\
54 \\
51 \\
\\
48 \\
45 \\
42 \\
59 \\
56 \\
\\
53 \\
30 \\
27 \\
24
\end{tabular} & \begin{tabular}{|c|} 
oent. \\
54 \\
52 \\
50 \\
48 \\
46 \\
44 \\
42 \\
40 \\
58 \\
56 \\
54 \\
52 \\
50 \\
28 \\
26 \\
24 \\
\\
22 \\
20 \\
18 \\
16
\end{tabular} & $\begin{array}{l}\text { m. } \\
18 \\
17,40 \\
16,80 \\
16,20 \\
15,60 \\
15 \\
14,40 \\
13,80 \\
15,20 \\
12,60 \\
12 \\
16,80 \\
16,50 \\
16,20 \\
15,90 \\
15,60 \\
15 \\
14 \\
15 \\
12\end{array}$ \\
\hline
\end{tabular}

S III. - Précis des règles générales de recetl.e des bois de mâture.

La force ou l'espèce d'un mât est généralement désignée par sa grosseur, et son prix est établi en conséquence.

Pour qu'il soit admis en recette d'après sa gros-

(1) La troisième classe comprend les bois de qualité inférieure. 
seur et au prix qui en résulte, il faut qu'il réunisse la longueur exigée et le proportionné qui forme le mât régulier.

La grosseur du mât régulier, ou son grand diamètre, ne doit pas être prise au pied de l'arbre, mais au sixième de sa longueur totale, point que l'on appelle le proportionné du gios bout.

Le mesurage du grand diamètre procède de 3 en 3 centimètres : la fraction de 15 millimètres et audessous est nulle; celle au-dessus de 15 millimètres est comptée pour 3 centimètres.

La Jongueur du mât régulier doit être, en mètres, le tiers du nombre de centimètres contenus au grand diamètre; elle se mesure à partir du pied de l'arbre, en négligeant la fraction de 15 centimètres et audessous, et en comptanl pour 3 décimètres celle audessus de 15 centimètres.

Le proportionné du mât, c'est-à-dire, son diamètre à la tête, se prend au point où finit la longueur du mât régulier; il doit avoir les deux tiers du grand diamètre, et se mesure par centimètre, en négligeant la fraction de 5 millimètres et au-dessous, et en comptant celle au-dessus de 5 millimètres pour un centimètre.

Un mât de 72 centimètres régulier est celui qui a 72 centimètres de grand diamètre mesuré à la sixième partie de sa lorgueur réglementaire en partant du pied de l'arbre, et qui a 48 centimètres de diamètre pris à sa longueur de 24 mètres.

Mais si ce mât n'a que 47 centimètres à 24 mètres 
de longueur, on descend le proportionné à 23 mètres de longueur; alors la pièce devient un mât régulier de 69 centimètres. Il est payé comme tel, en tenant compte des excédants, tant sur le grand diamètre qu'au proportionné, ainsi que de la longueur audelà de 23 mètres.

$\mathrm{I}^{\mathrm{re}}$ CLASSE. Mâts et vergues de hune. - II faut, pour remplir ce premier et essentiel service de la mâture, des mâts d'élite, sains, vigoureux, droits, sans nœuds préjudiciables à leur solidité, qui réunissent très-exactement la longueur et le proportionné exigés par le tableau de classement.

II $^{\mathrm{e}}$ CLasse. Mèches et jumelles supérieures. Quelques vices légers n'empêchehent p̂as les mâts de remplir ce service intéressant.

On entend par vices légers : $1^{\circ}$ Lorsqu'un mât est un peu courbé sur un sens, et qu'on peut, en le travaillant, le redresser sans perte;

$2^{\circ}$ Lorsqu'il a des nœuds petits et sains, mais trop fréquents, ou commençant trop bas pour permettre d'en faire un mât de hune, dont il aurait d'ailleurs la qualité ;

$3^{\circ}$ Lorsqu'il a de l'aubier assez épais pour faire craindre qu'en le travaillant il ne faille réduire le proportionné.

III ${ }^{\bullet}$ CLasse. Mèches et jumelles inférieures. L'impossibilité d'obtenir que les achats de mâture 
n'offrent que des mâts de $1^{\text {re }}$ et $2^{\mathrm{e}}$ classes oblige d'en recevoir de qualité inférieure, qui d'ailleurs remplissent, à peu de chose près, le même service pour les mâts d'assemblage.

C'est ici qu'est assignée la place de toutes les pièces qui ont quelques défectuosités.

On entend par défectuosités : $1^{\circ}$ Le mât un peu courbé sur deux sens, ou même assez courbé sur un seul sens pour être obligé de le redresser aux dépens du bois, ce qui en diminue la force;

$2^{\circ}$ Le mât chargé de nouds trop multipliés ou trop gros, ou dont quelques-ûns sont gâtés;

$3^{\circ}$ Celui dont la cime est, ou desséchée, ou dans le cas d'être réduite pour cause d'altération;

$4^{\circ}$ Le fil du bois tourné;

$5^{\circ}$ Les roulures, gercures, frottures peu considérables, après s'être assuré que le mal ne pénètre pas.

Mâts tronçonnés. - Les mâts tronçonnés sont ceux qui, se trouvant coupés par des nœuds réunis ou par des vices au-dessous de la longueur régulière, sont cependant capables, avec leur longueur réduite, de former des beauprés d'une seule pièce.

Mâtereaux. - Les mâtereaux de 48 à 36 centimètres doivent être d'une belle essence, droits, sans défauts, ayant au moins la longueur portée au tarif.

Menus mâtereaux. - Mêmes conditions que pour les mâtereaux. 
Qualités des mâts. - Le bon mât a le bois de couleur rouge pâle, les couches ligneuses égales, la substance résineuse suffisamment abondante et par couches régulières, le grain fin et serré, les fibres rapprochées et adhérentes, en sorte que, quand on entame la pièce, les copeaux s'en détachent sans sauter en éclats sous le coup de hache; et si on veut les désunir, ils se déchirent au lieu de se rompre. On observe que ces qualités se manifestent principalement dans les mâts de fraìche coupe, et ảiminuent dans les mâts de coupe ancienne.

Vices des mâts. - Les vices d'essence et même accidentels sont en général annoncés par la couleur blanche et rouge foncé du bois, par le défaut d'une quantité suffisante de substance résineuse, par des nœuds gros, multipliés et gâtés. - Ils sont confirmés par les taches, roulures et gélivures que l'on découvre aux deux bouts de la pièce. Ces vices sont principalement :

$1^{\circ}$ Quand l'arbre est couronné ou mort sur pied, ce qui se reconnait particulièrement au dessèchement de la tête du mât et à l'altération de toute sa substance;

$2^{\circ}$ Les roulures au cœur, qui permettent à l'eau de pénétrer dans l'intérieur de la pièce, et provoquent son èchauffement et sa pourriture;

$3^{\circ}$ Les gélivures simples et les cadranures qui, par une ou plusieurs fentes allant du cœur de l'arbre à la circonférence, rompent les fibres du bois et altèrent la force de la pièce; 
EMPLOI SPÉCIAL DES bOIS DE MATURE.

$4^{\circ}$ L'entr'écorce, la frotture, lorsqu'elles ont donné lieu à un principe de pourriture;

$5^{\circ}$ Des nœuds gros, fréquents, prenant du pied ou du tiers de l'arbre, se détachant avec facilité, ou pourris, ou en rosette c'est-à-dire dans la même ligne sur plusieurs sens. De pareils nœuds coupent le mât et le mettent le plus souvent tout à fait hors de service, quand même l'essence serait bonne;

$6^{\circ}$ Trop d'aubier, trop d'arc sur deux sens, même sur un seul, le cœur de l'arbre placé sur le côté. Ces trois vices obligeraient de réduire la pièce en la travaillant, au point de ne pas pouvoir compter sur sa solidité dans un mât d'assemblage (1).

\section{Article IV.}

Emploi spécial des bois de mâture.

Les mâts des vaisseaux, frégates, corvettes et bricks se composent généralement, dans leur ensemble, de quatre longueurs disposées les unes audessus des autres. Le bas mât qui s'implante à fond de cale, le mât de hune, le mât de perroquet et le mât de cacatois.

Différentes conditions essentielles de la mâture se combinent pour rendre obligatoire la construction de chacun des mâts supérieurs en une seule pièce de bois. Il faut qu'ils conservent toute leur flexibilité, que les vergues puissent glisser facilement contre

(1) Extrait de l'instruction du Ministre de la Marine, en date da 29 février 1848 . 
eux quand on les amène, et enfin que le mât luimême, lorsqu'on le guinde ou qu'on le cale (l'élève ou l'abaisse) ne soit arrêté par aucun obstacle dans son glissement à travers le trou du chouquet. (Le chouquet est une pièce de bois percée de deux trous, l'un carré et l'autre rond; le trou carré reçoit la tête du mât inférieur, le trou rond permet le passage du mât supérieur quand on le cale.)

Les bas mâts d'un bâtiment au-dessus du rang de brick ont des dimensions trop considérables pour permettre de les faire d'un seul arbre. On a recours alors aux mâts d'assemblage composés d'un plus ou moins grand nombre de pièces en épaisseur et en longueur. On comprendra d'autant mieux cette nécessité en remarquant qu'un bas mât de vaisseau de 120 canons a de 38 à 40 mètres de longueur et $1^{\mathrm{m}}, 06$ centimètres de gros diamètre; le diamètre au petit bout est les 2/3 du gros diamètre, c'est-à-dire, $0^{\mathrm{m}}, 70$ centimètres $(1)$.

(1) Le bas mât d'un vaisseau de 120 coûte environ 40,000 francs.

En 1859, le port de Cherbourg a reçu des Iles Vancouver plusieurs pièces de màture, essence Pin, dont le bois a le grain très-fin, les couches étroites et régulières, suffisamment chargé de résine et pas très-lourd. Ces arbres âgés en moyenne de 230 ans portaient les dimensions suivantes :

\begin{tabular}{|c|c|c|c|c|c|}
\hline $\begin{array}{c}\text { Longueur. } \\
56.90\end{array}$ & $\begin{array}{c}\text { Grand diamètre. } \\
0.92\end{array}$ & $\begin{array}{c}\text { Petit diamètre. } \\
0.7 \text { b̆ }\end{array}$ & $\begin{array}{c}\text { Prix. } \\
7,8 \div 9^{\text {f } 8}\end{array}$ & & $\begin{array}{l}\text { Cube. } \\
26.04\end{array}$ \\
\hline 36.60 & 0.90 & 0.76 & 7,74ว & . & 25.21 \\
\hline 36 : & 0.90 & 0.71 & 7,484 & - & 2304 \\
\hline 54.50 & 0.81 & 0.63 & 4,7905 & 0 & 17.88 \\
\hline 55. & 0.81 & $0.6 \mathrm{I}$ & $4, \check{6} 6$ & - & 16.65 \\
\hline 53. & 0.81 & 0.66 & 4,753 & , & 17.59 \\
\hline 50.50 & 0.78 & 0.62 & 4,089 & , & 1494 \\
\hline
\end{tabular}


DES BOIS EMPLOYÉS AUX EMMÉNAGEMENTS, ETG.

Pour composer un mât d'assemblage, on réunit plusieurs couches de bois et on a soin que, dans la longueur, les écarts des pièces qui entrent dans l'assemblage se croisent assez pour assurer la solidité de la construction. Ces pièces de bois se réduisent à la tête, suivant les dimensions du mât.

Les couches de bois étant parfaitement dressées au rabot, on les enduit d'une peinture épaisse et on insère entre les couches juxta-posées une série de dés ronds en bois dur, placés en échiquier, de manière à prévenir le glissement des pièces les unes contre les autres. Le mât bien arrondi, on consolide l'assemblage au moyen de cercles en fer forgé d'une pièce.

\section{$\Lambda$ RTICLE V.}

Des bois employés aux emménagements et à l'armement des vaisseaux.

Sans vouloir entrer ici dans des détails que ne comportent ni le but ni le cadre de cet ourrage, nous indiquerons brièvement l'emploi que l'on fait des principales essences, soit dans les emménagements de l'intérieur des vaisseaux, soit dans la construction ou la fabrication des objets d'armement.

Le chêne, nous l'avons déjà dit, est le bois dont on fait le meilleur merrain. La marine fait une consommation considérable de merrain de chêne, pour la fabrication des tonneaux qui servent à loger les 
liquides, les vivres, les objets d'habille ment, etc., etc. Le merrain que la marine emploie est débité suivant des dimensions spéciales, et provient en général du nord de l'Europe (1).

Le chêne s'emploie encore, concurrem ment avec le pin, le sapin (2) et l'épicéa, à tous les ouvrages de menuiserie, notamment à la construction des soutes, des chambres et logements d'officiers, etc., etc.

Le hêtre sert également à la menuiserie, mais il est peu employé.

(1) J'ai vu dans un de nos ports du merrain de Dantzick dont la longaille avait de $0^{\mathrm{m}}, 07$ à $0^{\mathrm{m}}, 08$ d'épaisseur, sur $0^{\mathrm{m}}, 1 \mathrm{~b}$ de largeur et $1^{\mathrm{m}}, 90$ à $1^{\mathrm{m}}, 93$ de longueur. Ces bois se vendent à Paris à peu près sur le pied de la planche échantillon. Ils sont très-recherchés pour la menuiserie, surtout pour les parquets, parce qu'ils sont tendres, faciles à travailler et peu disposés à se voiler, à se fendre ou à se gercer, quand ils sont mis en œuvre. En général, le bois de chêne des provinces de la Baltique ne vaut pas celui que nous produisons en France, et comme il importe d'employer du merrain de bonne qualité pour la constraction des tonneaux destinés à contenir des liquides (surtout les spiritueux) et à séjourner dans les cales, il semble que ce serait tout profit pour l'Etat de n'employer que du merrain de France, dût-il le payer un peu plus cher que celui de Dantzick.

(2) Le sapin et l'épicéa que l'on emploie dans les chantiers de la marine proviennent en grande partie des forêts de la Suède et de l'Illyrie. Les uns s'appellent bois blancs ou bois rouges de Suède, suivant l'essence, les autres sapins de Trieste. Pour tous les usages auxquels servent ces bois, on peut regretter, comme pour le merrain de chêne, que l'on demande à l'étranger des produits que nos sapinières fournissent en grande abondance, et dont la qualité a été reconnue supérieure, au moins en ce qui concerne les sapins de l'arrondissement de Limoux. La marine du commerce emploie même en mâture beaucoup de sapins provenant de nos belles forêts du Jura et des Alpes. 
Avecl'orme on fait des poulies (1), des galoches, des cabestans, des caps de mouton, des affûts de canon et, en général, tous les ouvrages qui exigent de la solidité et qui sont exposés au frottement. L'orme sert aussi à faire des canots et toute espèce d'embarcations à parois minces et légères, pour la construction desquelles il faut employer des bois qui ne soient sujets ni à se fendre ni à se déjeter.

Le frêne s'emploie à peu près exclusivement à faire des avirons (2).

(1) Les roues des poulies et des galoches sont ordinairement faites d'un bois exotique très-dur qu'on nomme bois de Gaïac.

(2) Je dois à l'obligeance de M. Schlumberger, ingénieur de la marine, tous les renseignements contenus dans ce chapitre sur l'emploi des bois dans les constructions navales. 


\section{CHAPITRE CINQUIEME.}

DES QOALITÉS ET DES DÉFAUTS DES BOIS D'OEOVRE.

Article I.

De la structure des bois.

Après avoir énuméré dans les chapitres précédents les qualités que l'on exige des bois de marine, et les défauts ou les vices particuliers dont doivent être exempts les bois destinés aux différentes parties de la construction d'un vaisseau, nous allons indiquer les moyens d'apprécier les qualités diverses des bois d'œuvre en général, nous ferons connaître ensuite la nature des défauts ou des vices qui portent atteinte à leur organisation.

Comme tous les corps organisés, les bois sont soumis à toutes les vicissitudes de la vie. Tant qu'ils végètent, ils sont exposés à des accidents et à des maladies qui peuvent altérer leur constitution et, quand ils sont morts ou abattus, ils se décomposent -plus ou moins lentement, selon leur qualité, sous l'influence de l'air, de la chaleur' et de l'humidité. Pour pouvoir apprécier les qualités diverses des bois et se rendre compte des causes d'altération et de 
destruction auxquelles ils sont exposés, il faut remonter à leur constitution analomique.

Le bois est intimement composé de cellulose (1) et de lignine. Il renferme en outre des principes minéraux qui, par la combustion, donnent de 1 à 5 p. 0/0 de cendres.

La cellulose constitue les membranes propres de tous les tissus élémentaires, dans les cavités desquels sont renfermés des principes divers, fécule, sucre, gomme, matières azotées éminemment fermentescibles.

La lignine ou ligneux est un principe toujours lié à la cellulose. C'est la matière qui incruste la cellulose des organes élémentaires pour constituer le bois.

"Le bois est formé de couches annuelles, dispo》) sées concentriquement autour de la moelle, dont „ les plus extérieures sont les plus récentes. Chaque ఎ couche ligneuse peut être formée de fibres, de "parenchyme ligneux, de canaux résinifères, de "vaisseaux disposés en faisceaux longitudinaux, ఐ) entre lesquels s'interposent les rayons médullaires " qui se dirigent de la moelle à l'écorce. Les fibres 》) et les rayons ne manquent jamais et constituent D souvent à eux seuls le bois (conifères). Les vais" seaux ne se rencontrent que dans les bois feuillus, „ les canaux résinifères que dans les conifères. Le

(1) La cellulose est un principe immédiat neutre, c'est-à-dire, dans lequel l'hydrogène et l'oxygène sont dans les proportions nécessaires pour former de l'eau. - La lignine est un principe surhydrogéné. 
》parenchyme ligneux fait souvent défaut. Tous ces

„ tissus ont leurs parois composées de cellulose. Ces

" parois de cellulose sont elles-mêmes incrustées

๖ plus ou moins de lignine.

DLes différents éléments du bois ne sont pas 》) toujours uniformément répartis et de forme con》) stante dans l'épaisseur d'une même couche; en 》 général, ils déterminent au bord interne de celle》 ci un tissu plus lâche et plus mou que celui du D bord externe. On appelle bois de printemps et bois D d'automne, ces deux régions habituellement diffé) rentes d'une même couche, en raison de la saison ๖ dans laquelle chacune d'elles s'est principalement „ développée.

๖ Les fibres constituent essentiellement la masse D du bois et ne manquent jamais; elles en forment „ la partie la plus compacte que l'on dirait pleine » à l'œil nu. Elles varient suivant les essences en D longueur et en diamètre; leurs parois peuvent D ètre plus ou moins épaisses, jusqu'au point d'obsఐ truer presque entièrement leur cavité centrale; ఐ elles sont tantôt uniformément réparties les unes ๖ à côté des autres, tantôt groupées en faisceaux » droits et parallèles entre eux ou plus ou moins „ ondulés. C'est en partie de ces circonstances que 》 résultent les bois durs et les bois téndres, ceux à » grain fin ou à grain grossier, ceux qui sont aptes ¿ à la fente et ceux qui y sont impropres.

„Les vaisseaux sont disséminés longitudinale» ment au milieu du tissu fibreux; ils ne se trouvent 
" pas nécessairement dans tous les bois. Ils man-

" quent complétement dans les conifères. Générale" ment d'un plus gros diamètre que les fibres et à "parois plus minces, ils sont le plus souvent visibles „ à l'œil nu, surtout sur une section transversale où D ils apparaissent sous forme de trous. Les vaisseaux D existent toujuiris dans les bois fevillus.

"Les canaux résinifères" s'observent chez beauD coup de conifères et manquent dans les bois pourఎ vus de vaisseaux dont ces canaux paraissent être " les représentants; ils sont composés d'une cavité " étroite, longitudinale, rayonnante et entourée "d'une couche de cellules d'une nature spéciale, " très-petites, délicates et serrées. A l'œil nu, ils " apparaissent sur la tranche du bois sous forme de "perforalions analogues à celles des vaisseaux; et " vus sur le fil, ils représentent de petites lignes lon" gitudinales brunes ou rougeâtres, en raison de la ఎ résine concrète qui s'y trouve accumulée.

"Les canaux résinifères sont très-apparents dans „ les pins et le mélèze; ils sont très-rares et peu "visibles dans l'épicéa; dans le sapin, le cèdre, les " genèvriers, l'if, on ne rencontre plus que quel" ques cellules résinifères disséminées dans le bois. - Les rayons médullaires apparaissent sur la " tranche du bois sous la forme de lignes rayon"nantes plus ou moins longues; suivant le fil, ils " déterminent des taches nacrées, des maillures plus D foncées ou plus claires que le bois. La forme, " la grandeur et le nombre de ces maillures dépen- 
" dent de la direction du débit, de la hauteur des

- rayons, de leur nombre. Elles sont les plus grandes

" possible, quand le bois est coupé dans la direction

ఐ des rayons eux-mêmes, c'est-à-dire, quand il est

1). débité sur maille; elles sont les plus petites possible

ఐ au contraire, quand le débit est perpendiculaire à

„ ces mêmes rayons.

" Les rayons médullaires sont plus ou moins „ apparents à l'œil nu, mais ils ne manquent jamais.

D Leur longueur est très-variable dans un même

" bois, mais leur direction est toujours perpendicu-

"laire aux couches qu'ils traversent.

"La couleur du bois est sujette à quelques varia»tions suivant le sol, l'exposition, l'altitude, le cli" mat. Tous les bois sont blancs dans leurs premières " années, même l'ébène. En général ils ne conservent

- pas cette couleur. Le dépôt de lignine qui, en n incrustant leurs tissus, les solidifie, et de l'état \ d'aubier les fait passer à l'état de bois parfait, est ๖ généralement accompagné d'un dépôt de matière " colorante, spéciale pour chaque espèce, de sorte "que souvent la coloration plus ou moins foncée " d'un bois est un indice d'une lignification plus n complète. Cependant il est des espèces dont la - lignine n'es! associée à aucune matière colorante, $\triangleright$ et dont la solidification ou transformation d'aubier \ en bois parfait se fait sans qu'il se produise de w changement de couleur; ces bois restent blancs : - charmes, érables, etc. Chez d'autres, il ne se pro") duit en quelque sorte jamais de bois parfait : bois „blancs. 
QUALITÉS ET DÉFAUTS DES BOIS D'OEUVRE.

"Dans les bois colorés, la transformation de - l'aubier en bois parfait se fait diversement. Ainsi " elle peut se produire au bout d'un temps plus ou ఐ moins long pour la même espèce, d'où résultent " des bois ayant beaucoup ou peu d'aubier. Cette ע transformation peut ensuite se faire à peu près " régulièrement, de sorte que la limite entre l'au") bier et le bois parfait est très-nette. Tels sont les " grands arbres à tige élevée, chênes, pins, mélèzes, » etc. (1)."

\section{Article II.}

Des causes qui influent sur la qualité des bois de même espèce et des caractères qui servent à la faire reconnaître.

La qualité d'un bois d'œuvre ne peut s'apprécier que relativement à l'usage auquel on le destine, et dépend toujours de la quantité de lignine qui incruste les tissus, de la structure des fibres, de la longueur des rayons, de la disposition et de la grosseur des vaisseaux et, s'il s'agit de bois résinenx, de la quantité de résine concrète dont ils sont imprégnés.

Les bois à vaisseaux gros, à fibres fortes et allongées et à croissance régulière, sont à la fois résistants et élastiques; tels sont le chêne, le châtaignier, le frêne, l'orme. Les bois résineux à fibres fortes et

(1) Extrait de la Description des bois, par M. Mathieu, professeur d'histoire naturelle à l'Ecole forestière. 
allongées jouissent des mêmes propriétés et sont d'autant plus tenaces et durables qu'ils renferment plus de résine dans leurs tissus. La résine, dans de certaines proportions, peut aussi en accroître l'élasticité.

Les bois à fibres allongées, droites et parallèles entre elles, sont les plus propres à la fente. Les principaux parmi les bois feuillus sont le châtaignier, le hêtre, le chêne et parmi les bois résineux l'épicéa, le pin sylvestre, le sapin.

Les bois les plus durs, les plus tenaces et les plus compactes ont la fibre courte, les rayons très-minces, les vaisseaux petits ou très-petits; ils ne sont pas propres à la fente, mais ils sont susceptibles d'un beau poli et conviennent surtout aux emplois dans lesquels le bois est exposé à un frottement continu, comme les dents de roues, les écrous, les vis, les manches d'outils, etc. Tels sont, parmi nos bois indigènes, le charme, les érables, les alisiers, les sorbiers, le pommier, le poirier, l'épine blanche et le cornouiller mâle.

Mais les qualités propres à chaque essence sont plus ou moins développées selon les conditions de climat, de sol et de traitement dans lesquelles les bois ont crû. Ces conditions n'influent pas dans le même sens sur toute espèce de bois; car, tandis que le pin sylvestre des plaines de la Russie a plus de densité et de ténacité que celui de notre pays, les chênes du midi de la France sont plus denses et plus durables que ceux de même espèce qui croissent 
dans les départements du nord; tandis que les bois résineux acquièrent plus de qualité dans les sols médiocres que dans les terrains très-substantiels, les chênes élevés dans des terrains fertiles et profonds ont une qualité de bois meilleure que celle des chênes de même espèce qui croissent dans des sols maigres; tandis que le chêne et le pin syjlvestre demandent pour prospérer à participer largement aux influences de la lumière, le sapin réclame la participation aux mêmes influences dans une mesure beaucoup moins large et acquiert une qualité de bois d'autant meilleure, qu'il croît dans une gêne plus grande, pourvu que cette gêne ne soit pas telle qu'elle s'oppose au développement régulier de sa végétation.

Toutefois, il n'est pas rare de rencontrer dans une même forêt des arbres de même espèce, soumis exactement aux mêmes conditions de végétation, ayant le mème port et les mêmes apparences extérieures, qui, lorsqu'ils sont abattus, présentent des différences sensibles dans la qualité de leur bois. D'où il résulte que, si on peut avoir des données assez précises sur la valeur générale des bois d'une forêt, on n'est jamais certain, tant qu'un arbre est sur pied, de porter un jugement exact sur la qualité de son bois. Nous nous bornerons donc à indiquer ici les caracières distinctifs de la qualité des bois abattus, mais en nous limitant aux essences qu'il importe surtout d'étudier sous ce rapport, à cause de leur emploi dans les grandes constructions, le chêne, le sapin et le pin sylvestre. 
Les bois de chêne sont de deux qualités principales, les bois durs ou nerveux, les bois tendres ou gras. Les caractères généraux qui servent à distinguer ces deux qualités du bois de chêne, tiennent à l'épaisseur des couches annuelles, aux fentes qui se manifestent après l'abatage par suite du dessèchement, à la coloration du bois et à l'odeur qu'il exhale.

Des bois de chêne nerveux, ceux qui ont le plus de qualité sous le rapport de la force, de la solidité, de la ténacité, de l'élasticité et de la durée, ont ordinairement les couches annuelles très-développées et variant d'épaisseur entre $0^{\mathrm{m}}, 005$ et $0^{\mathrm{m}}, 015$ et au-dessus. La partie extérieure de chacune de ces couches, qui comprend ce que l'on appelle le bois d'automne, est formée d'un tissu serré, plein, compacte, d'apparence cornée, tandis que la partie intérieure, ou bois de printemps, n'est que peu développée et ne présente qu'une zone étroite de vaisseaux qui apparaissent très-nettemeut sur la tranche sous la forme de petits trous très-rapprochés $\langle P l$. VIII, fiq. 1). Les bois de cette qualité ont les fibres fortes, serrées, difficiles à rompre ; les copeaux qui tombent sous la hache se cassent difficilement, et les morceaux ne se séparent que par déchirures. Lorsqu'on les rabote, la varlope enlève de longs rubans qui offrent une certaine résistance à la rupture, le bois est poli et brillant, et la fibre parait comme tapissée et incrustée d'une substance sirupeuse ou gommeuse toujours fraîche. 
Les bois de chêne nerveux prennent un retrait prononcé par le desséchement. Ce retrait se manifeste par des fentes plus ou moins fortes, profondes et nombreuses, que l'on remarque à la surface extérieure des pièces écorcées et sur la section d'abatage. Des fentes très-marquées sur la section d'abatage sont un indice des plus certains de la qualité du bois; mais, quand elles sont exagérées, elles peuvent constituer un véritable défaut. C'est ainsi que les chênes d'Italie, de Provence et d'Algérie éclatent quelquefois de manière à devenir impropres à toute espèce d'emploi comme bois d'œuvre (1).

La couleur n'est pas un indice bien certain de la qualité du bois, parce qu'elle s'altère facilement et assez promptement sous l'influence des agents extérieurs. Cependant, on remarque assez généralement que les bois de chêne de bonne qualité ont une.couleur à peu près uniforme qni devient seulement un peu plus foncée dans les couches voisines du cœur; de plus, le bois fraîchement abattu et qui n'a pas été

(1) Les chènes dont le bois est le plus dense sont : le tauzin, le liège, l'yeuse et le ballote qui n'est qu'une variété de l'yeuse et qui peuple de grandes forêts en Algérie. Le bois de ces chênes est plus nerveux, plus durable que celui du rouvre et du pédonculé, et peut être très-utilement employé dans certaines parties des constructions navales. Mais, outre que la tige de ces arbres a ordinairement peu de longueur et une conformation peu régulière, la fibre est toujours si serrée et le bois tellement disposé à se déjeter et à se gercer, que ces chênes ne peuvent supporter la comparaison, ni comme bois de travail, ni comme bois de construction, avec le rouvre et le pédonculé des climats doux et tempérés de France. - On remarque aussi, généralement, que plus un chêne a d'aubier, plus le bois parfait est dur et nerveux. 
flotté, a ordinairement une couleur jaune tantôt blanchâtre, tantôt brunâtre, tantôt rosée; mais le caractère tiré de la couleur ne doit s'observer que sur une section faite à quelque distance des racines, parce que dans la partie inférieure de la pièce, dans la culée, le bois peut être coloré diversement par les substances étrangères que charrie la séve.

Le chêne a une odeur sui generis qu'il est assez difficile de définir, mais que l'on reconnaît facilement quand on a fréquenté les coupes en exploitation et assisté à la découpe et au débit des bois. Cette odeur aigre et amère est d'autant plus forte que le bois est plus sain, plus vigoureux, mieux nourri, et c'est un signe à peu près certain de sa bonne qualité et de sa solidité.

Le bois de chêne nerveux est propre à tous les usages auxquels on destine cette précieuse essence; mais il doit être recherché surtout pour les emplois qui exigent de la solidité, comme la charpente dans les constructions civiles et les pièces qui forment la membrure des vaisseaux dans les constructions navales. Sous ce rapport, le chêne péúunculé paraît avoir l'avantage sur le chêne rouvre de France.

Par opposition au bois de chêne nerveux, on appelle bois gras celui dont le tissu est mou, poreux, peu résistant, dont le grain est peu serré, la fibre lâche et imparfaitement lignifiée. Dans les bois gras les couches annuelles sont ordinairement peu développées, et le bois de printemps occupe dans chaque 
couche une place d'autant plus grande, par rapport au bois d'automne, que le chêne lui-même a été moins bien nourri $(P l . V I I I, f i g .3)$. Il suit de là que le bois a d'autant moins de force que les couches concentriques sont moins développées et que les vaisseaux de la couche de bois de printemps sont plus nombreux' et plus ouverts. Les chênes de la moins bonne qualité sous ce rapport n'ont presque pas de bois d'automne, et cette partie de la couche annuelle est si peu compacte qu'on la confond avec le bois de printemps; sur la tranche, les couches annuelles paraissent dans toute leur largeur percées de trous ou de vaisseaux comme un crible. - Le bois gras a la fibre sèche et cassante; les copeaux qui tombent sous la hache se rompent avec facilité et netteté, sans éclats ni déchirures; ceux qu'on détache avec la varlope, au lieu de former de longs rubans, se séparent sous l'outil en petites plaques. - Le bois de chêne gras prend peu de retrait par le dessèchement, et par conséquent n'est pas sujet aux grosses fentes comme le bois nerveux. - La couleur du bois fraîchement coupé est ordinairement brune, tirant sur le jaune fauve, le roux, ou le violet, d'un aspect terne et sec, souvent tachetée ou diversement veinée. Mais, en se desséchant, le bois prend une couleur plus claire, et, lorsqu'il est bien traité et mis à couvert en lieu sec et aéré, sa teinte devient plus belle et se rapproche souvent de celle du bois nerveux. - L'odeur du chêne gras est moins forte, moins acide, plus douceâtre que celle du chêne nerveux. 
Les bois gras absorbent très-facilement l'humidité de l'atmosphère et sont très-accessibles à la fermentation et à la pourriture, dès qu'ils sont exposés aux injures de l'air ou placés dans un milieu chaud et humide. Cette disposition des pièces à s'altérer promptement dans toutes leurs parties, fait considérer les bois de cette qualité comme absolument impropres aux constructions navales et à tous les emplois qui exigent de la solidité, de la force et de la durée. Cependant le bois gras, lorsqu'il est à couvert et dans un lieu sec, résiste assez longtemps et peut utilement servir à des emplois où le bois ne supporte ni charge ni fatigue. C'est ainsi que les chênes gras ou tendres, quand ce défaut n'est pas exagéré, sont les plus recherchés pour les beaux ouvrages de menuiserie et d'éhénisterie, parce que le bois est plus facile à travailler et qu'il n'est sujet ni à se fendre, ni à se voiler, ni à prendre du retrait, quand il est mis en œuvre. A ce point de vue, le chène rouvre de qualité ordinaire parait avoir l'avantage sur le chêne pédonculé, du moins si on en juge par ce qui se passe dans les pays accidentés où les menuisiers et les fabricants de merrain accordent une préférence marquée aux chênes de montagne ou de côteau qui sont ordinairement de cette espèce. Toutefois il y a des chênes rouvres dont le bois est très-nerveux, comme il y a des chênes pédonculés dont le bois est mou, poreux, gras.

Entre les deux types extrêmes de la qualité des bois de chêne, il existe une infinité de nuances qui 
se rapprochent plus ou moins des bois nerveux ou des bois gras et qu'il serait impossible de définir par des caractères bien tranchés $(P l$. VIII, fig. 2). Mais ces nuances sont faciles à apprécier pour qui veut les examiner, et comparer entre eux les bois de chênes qui ont crû dans des conditions différentes de sol ou de climat (1).

Le sapin est, de tous les bois résineux, le plus commun en France et le plus employé, soit dans les constructions, soit dans les ouvrages de menuiserie; mais on n'apporte généralement pas, dans le choix des sapins qu'on met en œurre, la même attention qu'on donne au chêne, parce qu'ils ne servent pas à des usages aussi importants que celui-ci. Cependant, soit qu'on l'emploie comme charpente, soit qu'on le destine en menuiserie à des ouvrages qui exigent de la solidité et de la durée, il n'est pas indifférent de négliger la qualité du bois, attendu que la durée d'une charpente de sapin peut varier beaucoup, suivant que le bois est de qualité médiocre ou supérieure. Le sapin de bonne qualité se distingue facilement à ses couches annuelles régulières, peu développées, et présentant sur la tranche un grain fin, serré, plein. Le sapin de qualité médiocre, au contraire, a souvent les couches concen-

(1) Les bois de chêne de qualité moyenne, qui ne sont ni très-nerveux ni très-tendres, sont réputés les meilleurs pour la fabrication des futailles, à cause de l'influence qu'ils exercent sur la conservation et la bonification des vins. 
triques très-larges, et son bois est formé d'un tissu mou, poreux, spongieux.

Il en est de même de l'épicéa et du pin sylvestre, dont la qualité s'apprécie de la même manière, d'après la régularité et l'épaisseur des accroissements annuels du diamètre, et d'après l'aspect plus ou moins compacte du bois vu sur une section perpendiculaire à l'axe. Mais, tandis que le sapin et l'épicéa n'ont pas d'aubier, ou du moins que l'on ne fait aucune distinction dans leur emploi entre le bois du cœur et celui des couches extérieures, le pin sylvestre a un aubier très-marqué, souvent trèsdéveloppé, dont le bois diffère essentiellement, sous le rapport de la qualité, de celui du cœur ou du bois parfait. Le bois aubier du pin sylvestre est blanc jaunâtre et se distingue très-facilement du bois parfait qui est brun rougeâtre, ou jaune rosâtre. Le bois aubier du pin se dessèche, s'altère et se décompose promptement à l'air; il est surtout exposé à la vermoulure; tandis que le hois parfait est extrêmement solide, résistant, élastique et aussi durable, si ce n'est plus, que les meilleurs bois de chêne. Sous le rapport de la solidité, de l'élasticité et de la durée, le pin sylyestre est (avec le mélèze et le laricio) infiniment supérieur aux autres bois résineux que nous produisons en France; mais la nécessité où l'on est de purger ce bois de son aubier pour certains emplois, et par conséquent de réduire beaucoup les dimensions utiles des pièces, constitue 
le pin sylvestre dans un état d'infériorité réelle, comme bois d'œuvre, vis-à-vis du sapin ou de l'épicéa.

ARticle III.

Des principaux vices ou défauts qui se rencontrent dans les bois d'œuvre.

\section{SI. - Généralités.}

Ce que nous venons de dire suffit pour apprendre à distinguer les différentes qualités de bois de chêne, de sapin et de pin sylvestre. Cetle distinction est importante à faire lorsqu'il s'agit de bois de grandes constructions et spécialement de bois de marine, car il est constant que la plus ou moins bonne qualité des bois qui entrent dans la construction d'un vaisseau peut faire varier du simple au double la durée du bâtiment. Cette importance sera plus frappante encòre lorsqu'on saura que la marine militaire emploie annuellement environ 40,000 mètres cubes de bois de chêne équarris, qu'il entre à peu près 5,000 mètres cubes de bois dans la charpente d'un vaisseau de premier rang, et que le prix de revient de la construction seule ne coûte pas moins d'un million et demi à l'Etat.

Mais, après avoir fait connaître les caractères généraux d'après lesquels on apprécie la qualité des bois abattus, il nous reste à parler des défauts ou vices particuliers qui peuvent affecter tous les bois, 
particulièrement les bois de chêne, et faire rejeter des constructions ceux mêmes qui, par leur constitution, seraient de la meilleure qualité. Ces vices ou défauts sont :

La torsion des fibres;

La gélivure:

La roulure;

La cadranure et la pourriture;

La grisette, les nøuds, les taches du bois;

La lunure;

La frotture, l'entr'écorce, les trous de vers;

\section{S II. - De la torsion des fibres.}

Les bois à fibres torses, que l'on appelle aussi bois tors ou bois virants, sont ceux dont les fibres, au lieu d'être parallèles à l'axe de l'arbre, décrivent autour de lui des hélices plus ou moins allongées. Ces bois sont moins résistants et moins élastiques que les bois à fibres droites, mais ce défaut, quand il n'est pas excessif, n'empêche pas que les bois ne soient utilement employés dans les constructions civiles. La marine ne considère pas non plus la torsion des fibres comme une cause suffisante de rebut pour les bois courbants (l'étrave exceptée), mais elle l'exclut absolument de tous les bois droits et de toutes les pièces qui peuvent être débitées en bordages. On conçoit en effet qu'un bois virant ne peut donner que de mauvais bordages, parce qu'en le débitant à la scie, il est impossible que les fibres ne soient 
pas coupées à divers endroits, ce qui diminue considérablement la force du bois. Débités en planches du commerce, les bois tors ne fournissent aussi que de mauvais sciages; ils sont d'ailleurs essentiellement impropres à la fente.

Les bois dont la fibre est torse sont faciles à reconnaître tant que l'arbre est recouvert de son écorce, mais quand ils sont débités en planches, ou simplement équarris, il faut une certaine habitude pour distinguer un bois virant de celui qui a les fibres droites.

\section{§III. - De la gélivure.}

La gélivure consiste dans une crevasse ou fente longitudinale qui va de la circonférence de l'arbre vers le centre, à des profondeurs variables, et dont la cicatrice forme extérieurement un bourrelet qui reste toujours visible $(P l . I X, f i g .1)$. La gélivure rompt les fibres du bois dans la direction des rayons médullaires et, quand elle s'étend beaucoup en longueur et en profondeur, elle altère la force du bois au point que la pièce ne peut plus être employée dans les grandes constructions, même dans son entier. Si au contraire la gélivure s'étend peu, la pièce peut encore servir à certains usages comme charpente, mais on préfère généralement débiter les bois gélifs en planches ou en merrain.

La fente de la gélivure a presque toujours ses deux parois atteintes de pourriture, par suite de l'infil- 
tration des eaux pluviales ou d'un écoulement de séve; elle n'en devient alors que plus dangereuse, et il faut qu'on puisse l'enlever complétement pour que la pièce soit utilisable en marine. Nais une gélivure peu développée peut être tolérée dans les pièces de marine qui ne sont pas destinées au débit.

On pense que la gélivure est ordinairement produite par une contraction ou une dilatation inégale dans les fibres du bois, soit par suite des gelées et des vents froids, soit par l'action intense du soleil. La fente se recourre ensuite de hon bois et, par conséquent, ne règne pas toujours du centre à la circonférence.

Duhamel prétend aussi qu'il a produit des gélivures dans le corps de jeunes arbres en les pliant et les forçant beaucoup, de la même manière que pourrait le faire un grand vent ou un poids considérable de givre. ll a observé que la gélivure et la roulure se trouvent souvent réunies dans un même corps d'arbre. - Les coups de marteau peuvent aussi produire des fentes analogues à la gélivure.

$$
\text { SIV. - De la roulure. }
$$

Dès qu'un arbre est abattu, on remarque souvent sur la taille d'abatage une solution de continuité entre deux couches concentriques contiguës, de façon que ces couches ne sont point adhérentes. Cette fente circulaire constitue ce que l'on nomme 
une roulure $(P l .1 X, f i g .2)$. Quelquefois la roulure n'est que partielle, mais souvent elle forme un cercle complet, et alors l'arbre présente un cylindre creux de bois qui renferme un autre cylindre plein. Lorsque ce défaut règne sur toute la longueur de l'arbre et apparaît au pied et à la tête, la pièce est tout à fait défectueuse et il est rare qu'on puisse s'en servir comme bois d'œurre. Mais assez souvent la roulure ne s'étend pas à une très-grande hauteur et n'a lieu qu'au pied. Dans ce cas, la pièce alteinte de roulure peut encore être d'un bon service, lorsqu'elle est employée dans son entier.

Néanmoins, comme l'humidité qui s'introduit et séjourne toujours dans la roulure peut être une cause de plus prompt dépérissement, et que d'ailleurs ce défaut est toujours un obstacle pour le débit, une pareille pièce ne peut être employée dans les grandes constructions civiles ou navales que dans des conditions toutes spéciales, ou bien, si la roulure s'étend peu, qu'après une réduction de sa longueur.

La roulure est, comme la gélivure et la torsion des fibres, un défaut ou un accident, et non une maladie du bois; mais la roulure ne se manifeste par aucun signe extérieur, tant que l'arbre est sur pied, et ne se montre que sur la tranche du bois abattu. La roulure n'est même pas toujours immédiatement apparente sur la taille d'abatage; mais elle devient très-évidente, au bout de peu de temps, quand le bois est suffisamment ressuyé. 
On constate souvent plusieurs roulures dans la même section d'un arbre, et l'on remarque généralement que plus elles sont nombreuses, petites, rapprochées et superposées, moins elles s'étendent dans la hauteur de la pièce; tandis qu'une roulure isolée, peu distante du cœur, circulaire et trèsmarquée, s'étend assez souvent jusqu'à l'extrémité opposée, et apparaît de la même manière aux deux bouts de la pièce.

Les couches ligneuses se formant entre l'écorce et le bois, on attribue la roulure à toutes les causes qui peuvent occasionner la séparation de l'écorce d'avec le bois. Parmi ces causes, Duhamel cite en première ligne le vent. "Il est sensible, dit-il, que "lorsque le vent agite et plie en différents sens les " jeunes arbres, leur écorce qui n'est presque pas " adhérente au bois peut s'en séparer dans quelques ¿ points, surtout quand les arbres sont en séve et v chargés de leurs feuilles. - En hiver, le poids du " givre peut produire le même effet malgré l'adhé" rence de l'écorce au bois. Ce défaut peut encore ఎ être produit par les voitures dont les moyeux 》 endommagent l'écorce et donnent lieu à une v roulure ordinairement peu grave qu'on nomme ¿ frotture. ")

Duhamel termine ses observations en affirmant qu'il a produit artificiellement des roulures :

$1^{\circ}$ En détachant l'écorce du tronc d'un arbre et en la remettant à sa place. Ce morceau d'écorce ainsi replacé s'est greffé avec celle qui était restée adhé- 
rente au bois, il s'est formé de nouvelles couches ligneuses; mais à l'endroit où l'écorce avait été séparée du bois, il est resté une solution de continuité, autrement dit une roulure;

$2^{\circ}$ En pliant bien fort de jeunes arbres dont il voulait rompre une partie du corps ligneux, il a occasionné dans leur intérieur des roulures qu'il a retrouvées quelques années après, quoique les plaies extérieures eussent été parfaitement cicatrisées.

Il a observé, enfin, que les baliveaux élevés dans un taillis sont plus sujets à être roulés que les arbres venus en massif et aussi que ceux qui ont crû isolément en plein air.

D'autres naturalistes prétendent que la roulure doit être attribuée exclusivement à la gelée qui, en détruisant le cambium sur certains points de l'arbre, occasionne la séparation en ces points de l'écorce d'avec le bois.

Si cette explication était exacte, tous les arbres de même âge, dans une même forêt, ayant subi les mêmes influences, devraient se trouver roulés de la même manière, et la position de la roulure indiquerait exactement l'année dans laquelle la gelée précoce aurait eu. lieu. Or, on a constamment observé le contraire sur des arbres roulés de même âge et placés dans les mêmes conditions climatériques.

Enfin, on dit que la roulure provient aussi quelquefois du choc qu'éprouvent les arbres en tombant, lors de l'abatage. Ce fait est difficile à constater, 
mais ce qui est certain, c'est que les coups de marteau dont on frappe les arbres réservés dans les coupes produisent très-fréquemment des roulures partielles ( $P l . X I 1, f i g .1)$.

\section{§V. - Cadranure et pourriture.}

Lorsqu'on abat un vieux chêne, la couleur du bois qui entoure le canal médullaire a souvent une teinie birunâtire plus foncée que celle des parties plus éloignées du cœur. Si en même temps les couches de bois du centre paraissent comme écrasées par la compression de celles qui les entourent, et si elles semblent plus chargées d'humidité, c'est un signe que le bois du cœur est moins bon que celui des couches qui l'enveloppent, qu'il a déjà subi une altération dans sa qualité propre, et que l'arbre luimême était dépérissant. Cette décomposition du cœur des vieux chênes, effet naturel de la décrépitude, se manifeste souvent, sur une section faite à la base de l'arbre, par des fentes qui partent du cœur et se dirigent vers lá circoniférence comme les rayons d'un cadran. De là, le nom de cadranure sous lequel on désigne, d'une manière générale, le vice que nous venons de décrire, soit qu'il apparaisse au pied, soit qu'il se manifeste au petit bout d'une pièce de chêne, ou même dans le cœur des branches $(P l . X$, fig. 1).

Quand le mal n'est pas ancien, la cadranure n'existe souvent qu'au pied de l'arbre, et alors il 
suffit de le tronçonner à une certaine hauteur pour pouvoir employer la partie saine comme bois d'œurre dans son entier. Mais quand les bois sont cadranés dans toute leur longueur, ils ne sont plus propres aux constructions, parce qu'ils ont déjà perdu une grande partie de leur force et que le commencement d'altération ou de pourriture dont ils sont atteints ne tarde pas à faire des progrès rapides.

Les fentes de cadranure ne peuvent être confondues avec les fentes de gélivure, ni avec celles que l'on remarque fréquemment sur la tranche des bois nerveux $(P l . V I I I, f i g .1)$ et qui proviennent du retrait que prend le bois en se desséchant. Les premières ont un aspect noirâtre et ne s'étendent jamais au-delà des couches de bois cadrané dont le tissu est mou, quelquefois friable, et répand d'ailleurs une odeur de pourri facile à reconnaitre. Souvent aussi la cadranure ne se manifeste au pied de l'arbre que par une seule fente ouverte et noirâtre, mais si on tronçonne la pièce à une petite distance du pied, les fentes augmenteñi en numbre et en ouverture, puis diminuent un peu plus haut pour augmenter encore.

Au début, la cadranure n'est que le signe d'une maladie qui fera bientôt des progrès rapides dans l'arbre abattu, si on l'emploie dans son entier, et qui eût abouti à la pourriture complète et à la mort de l'arbre si on l'avait laissé plus longtemps sur pied. 
La pourriture que l'on appelle pourriture rouge ou simplement le rouge, à cause de sa couleur (brun-canelle), provient, comme la cadranure dont elle n'est que le développement, soit de la décrépitude des arbres surannés, soit d'un accident ou d'une maladie qui atteint l'un des organes principaux de la vie du végétal, avant qu'il soit parvenu à maturité. Quand le mal est ancien, le bois qui en est atteint devient cassant, friable, et finit par se réduire, dans l'intérieur même de l'arbre, en poussière fine qui ressemble à du tabac d'Espagne. Les arbres les plus vigoureux en apparence sont quelquefois en proie à cette maladie qui les ronge intérieurement, mais ce sont les bois tendres, à végétation lente, ceux surtout qui croissent dans des terrains sans profondeur, qui y sont le plus exposés. Souvent donc ce mal n'offre pas de signe extérieur apparent, d'autres fois il se manifeste au pied de l'arbre, entre le point d'attache de deux fortes racines, par des trous d'où l'on voit sortir une poudre grossière et brune semblable à celle de la chicorée à café; mais, en général, quand la maladie existe à un degré quelque peu avancé dans la partie inférieure d'un arbre, on peut presque toujours la reconnaître au son creux que rend le tronc, lorsqu'on le frappe avec un marteau ou le dos d'une hache. Toutefois, ce son, qui indique toujours que le bois est altéré ou que l'arbre est creux à l'intérieur, ne peut pas faire préjuger d'une manière certaine la présence de la pourriture rouge, 
DES BOIS D'OEUVRE.

parce qu'il existe d'autres maladies ou vices qui peuvent produire le même effet dans le corps d'un arbre sur pied : tels sont la pourriture blanche et la grisette dont nous parlerons plus tard.

La pourriture blanche peut être occasionnée par différentes causes, et se montrer dans toutes les parties du corps et des branches d'un arbre; mais on la qualifie différemment suivant sa provenance et le danger qu'elle présente pour les parties saines de bois qui se trouvent en contact avec elle. Celle dont nous voulons parler ici, et qui est plus spécialement connue sous le nom de pourriture sèche, est de toutes les pourritures blanches la moins contagieuse. Elle prend sa source au pied ou dans les racines de l'arbre, s'attaque ordinairement au cour comme la cadranure et pénètre dans la tige où on la voit finir en pointe à des hauteurs peu considérables et que l'on peut mesurer en sondant la pièce avec une tarière. Ce vice transforme le bois qu'il atteint en une substance sèche et molle, cotonneuse, filandreuse, facile à désagréger, de couleur jaune pâle passant successivement au blanc mat à mesure que le mal devient plus ancien; mais, pendant longtemps, il reste circonscrit dans un nombre limité de couches annuelles, sans s'étendre dans les parties environnantes dont le bois ne parait nullement souffrir du contact ou du voisinage des couches viciées. Les chếnes qui croissent en terrain maigre, et les vieux arbres élevés sur souche sont 
plus particulièrement exposés à ce genre de maladie (1).

Les chênes atteints de la pourriture blanche ne peuvent être employés dans les constructions qu'après réduction de la partie viciée et appartiennent plus habituellement à la catégorie des bois gras ou tendres.

\section{SVI. - Grisette, næuds, taches du bois.}

Lorsqu'une des grosses branches qui forment la tête d'un arbre ou qui partent de son tronc vient à mourir et se brise, ou bien si l'une de ces branches encore en vie est rompue par le vent, cassée ou coupée par un délinquant, ou retranchée par un élagage inintelligent, il reste au tronc un chicot ou moignon plus ou moins long dont les fibres pénètrent jusqu'au cœur de l'arbre, et dont l'autre extrémité se termine par un faisceau d'esquilles. Celles-ci s'altèrent promptement, absorbent l'humidité atmosphérique, retiennent les eaux pluviales, à la manière d'une éponge, et déterminent leur infiltration dans le chicot et dans le corps de l'arbre lui-même, suivant la direction des fibres. Cet accident constitue ce que l'on nomme une gouttière, et se rencontre fréquemment dans les vieux arbres et surtout dans les chênes couronnés.

On nomme abreuvoir une espèce de gouttière qui

(1) Les sols trop substantiels produisent souvent aussi le mème effet sur le pin sylvestre. 
se forme aux aisselles des branches, lorsque celles-ci, par les grands vents ou par le poids de la neige ou du givre, se délachent partiellement du tronc. La blessure, tout en se cicatrisant, présente une cavité dans laquelle les eaux s'amassent, et d'où elles finissent par s'infiltrer dans l'intérieur de l'arbre.

Les gouttières et les abreuvoirs produisent ordinairement dans le bois d'un chêne un vice ou une maladie que l'on nomme la grisette, et qui n'est autre chose qu'une décomposition plus ou moins avancée du tissu ligneux.

Plus le bois est tendre, plus les eaux de gouttière s'infiltrent facilement et déterminent rapidement la fermentation et la pourriture. Mais le danger que présente toujours une gouttière est plus ou moins grand, selon les circonstances dans lesquelles l'accident a eu lieu.

Si une branche arrachée, coupée ou rompue, n'est pas de trop forte dimension, il arrive souvent que la plaie se recouvre en peu d'années d'une couche de bon bois, et se trouve ainsi à l'abri des influences extérieures. L'affluence de la séve peut alors arrêter les progrès de la pourriture dont le chicot peut être déjà attaqué, et la branche seule continuera à se décomposer lentement, sans grand danger pourla partie du corps de l'arbre qui la renferme. Le bois attaqué se transforme en une substance blanche ou blanche jaunâtre, molle, filandreuse, spongieuse, complétement inodore, et forme 
dans le corps de l'arbre un amas de bois décomposé qui constitue ce que l'on nomme un nceud blanc ou une huppe. Comme le nœud blanc est enveloppé entièrement de bois sain, on en purge facilement la pièce qui ne perd de sa valeur qu'en raison de la grandeur de la poche que l'on a dû faire pour enlever le bois décomposé.

Mais si la plaie d'une branche cassée ou arrachée n'a pu être recouverte et protégée contre les agents extérieurs, la maladie progresse d'une manière continue et pénètre de plus en plus dans l'intérieur de l'arbre, soit en suivant les fibres de la branche qui peuvent l'amener jusqu'au cœur de l'arbre, soit en envahissant les fibres longitudinales du tronc, au-dessus et au-dessous de la branche, jusqu'à des distances souvent très-considérables. Dans ces circonstances, la présence du vice dans le corps de l'arbre se manifeste assez souvent sur la taille d'abatage par des taches, ou à l'extérieur sur l'écorce par le suintement d'une liqueur noirâtre, ou enfin, sous l'écorce, sur le fil du bois par des veines de couleur rousse, grisâtre ou brunâtre, de telle sorte qu'en suivant la trace de ces taches on peut toujours remonter à l'origine et au foyer de la grisette. Ces taches et ces veines reçoivent le nom de flammes de grisette (Pl. XI, fig. 1).

On peut également préjuger de l'intensité d'une grisette d'après la couleur du bois sur la section des branches ou des nœuds rez-tronc. Ainsi la section d'un nœud ou d'une branche attaquée par une 
grisette encore peu avancée, affecte une teinte brunchocolat très-caractéristique, et en travaillant le bois on s'aperçoit facilement qu'il n'a plus de nerf et qu'il est déjà décomposé. Dans cet état, le vice ne pénètre généralement pas fort avant et n'a encore attaqué que la branche. - Dans un degré plus avancé, le bois d'une branche grisettée est parsemé de points noirs et de points blancs; ceux-ci sont produits par la section de fibres complétement décomposées $(\boldsymbol{P l} . X I$, fig. 2). C'est alors ce que l'on nomme une grisette blanche ou vive, l'un des vices les plus dangereux du chêne, à cause de la rapidité avec laquelle il se propage, même après l'abatage et l'emmagasinage de la pièce. - Quelquefois aussi les filets ou les points blancs sont remplacés par des filets ou des points de couleur jaune orange, qui semblent indiquer un degré de pourriture encore plus avancé. Dans ce cas, le vice pénètre généralement jusqu'au cœur, les fibres longitudinales du tronc sont atlaquées jusqu'à une grande distance de la branche et le bois décomposé a souvent une odeur fétide et nauséabonde. - Mais si la section d'une branche rez-tronc présente une surface noire d'un tissu serré et sans odeur particulière, on est presque certain que le nœud est sain. C'est ainsi que les ouvriers disent que les nœuds noirs ne sont nullement dangereux et dénotent même une qualité supérieure du bois.

Dès qu'un arbre est abattu, il importe donc de rabattre rez-tronc toutes les branches, bosses ou lou- 
pes, et de visiter tous les nouds, même les plus petits et les moins suspects, avec la tarière et la gouge, si on veut être parfaitement sûr de la qualité du bois. Si un vice est signalé, on le sonde dans la direction de la branche pour en constater la profondeur, puis on s'assure s'il n'a pas fait de ravages dans le corps de l'arbre en entaillant le bois audessus et au-dessous du trou de sonde. Si le mal s'étend, au-dessus ou au-dessous de la branche, à une profondeur considérable, il ne reste plus qu'à tronçonner l'arbre à une distance convenable du foyer du vice, afin de s'assurer si le cœur lui-même n'est pas attaqué, et si les filets de grisette se sont suffisamment assainis pour que la pièce puisse servir comme bois d'œurre dans les grandes constructions civiles ou navales. Lorsque le vice n'a pas encore envahi tout le corps de l'arbre et se trouve concentré, comme cela arrive souvent, dans la région du cœur, on peut utiliser toutes les parties saines de la pièce, après les avoir purgées de tout le bois grisetté, en les débitant en sciages, merrain, etc.

Dans la marine, les arbres qui renferment des nœuds ne peuvent être admis en recette qu'après qu'ils ont été sondés et purgés de tout le bois gâté qu'ils pouvaient renfermer. Si les trous de sonde sont peu considérables, la pièce est admise sans réduction sur l'équarrissage; s'ils sont profonds et de nature à diminuer la solidité de la pièce, celie-ci peut être rebutée, ou réduite dans les dimensions de son équarrissage en proportion de la grandeur des trous de sonde. 
D'après ce que nous avons dit, on comprendra qu'une grisette peut se produire dans un arbre sans qu'elle soit necessairement due à un abreuvoir ou à une gouttière. Un épanchement de séve dans la fente d'une roulure ou d'une gélivure produit quelquefois des effets analogues à ceux d'une gouttière, et il n'est pas rare de voir les parois de ces fentes atteintes de griseltes, ce qui se reconnaît à la teinte noire que prend le bois malade et à l'odeur qu'il exhalc. Les taches que l'on remarque assez fréquemment sur la taille d'abatage des chênes ne proviennent pas toujours non plus d'une goultière; souvent elles sont dues à la présence d'une racine malade ou à des sucs co'orés que charrie la séve. De couleur ordinairement plus foncée que les taches de gouttière, les taches de racine affectent toutes les nuances entre le gris et le noir; elles vont en diminuant d'étendue à mesure qu'elles s'élèvent dans l'arbre et finissent toutes en pointe, à des hauteurs variables, mais que l'on peut assez facilement préjuger, soit en éboutant l'arbre à quelques centimètres du pied et en voyant dans quelle proportion elles diminuent de largeur sur la nouvelle section, soit en sondant le bois taché avec une tarière. Quelle que soit leur couleur, à moins que leur étendue ne soit exagérée, ces taches n'empêchent pas que le bois ne soit employé dans les constructions ordinaires; mais la marine ne reçoit pas de bois tachés, parce qu'en raison de l'emploi qui en sera fail, on ne peut rien fonder de certain sur leur solidité et leur durée. On remarque en effet 
que le tissu des parties tachées est presque toujours moins résistant, et absorbe plus facilement l'humidité atmosphérique que le bois environnant; ce qui fait dire que, même dans les constructions ordinaires, il est prudent de ne placer les bois tachés qu'à l'abri des influences extérieures.

\$ VII. - Lunure.

De tous les défauts qui peuvent affecter la qualité des bois de chêne, la lunure est un de ceux qui se présentent le plus fréquemment et qui méritent le plus de fixer notre attention. La lunure ou lune apparait, sur la tranche du bois, sous la forme d'un cercle $(P l . X$, fig. 2), ou quelquefois d'un arc de cercle, formé de plusieurs couches annuelles de couleur plus foncée ou plus claire que celle du bois environnant, et dont le tissu est atteint d'une maladie qui le rend plus particulièrement accessible à la pourriture et à la vermoulure.

Duhamel qualifiait ce défaut de double aubier et lui attribuait une grande importance, "parce que, " disait-il, le double aubier, qui est souvent de plus " mauvaise qualité que le vrai aubier, tombe bientôt ఐ en pourriture; ce qui fait qu'un arbre luné n'est " pas même bon à être employé dans son entier ni, " à plus forte raison, propre à être débité en bois de " sciage et de fente."

Quand un chêne est luné, la maladie apparaît presque toujours de la même manière aux deux ex- 
trémilés de chaque partie du tronc découpé en pièces de bois d'œurre et, suivant que la couleur du bois luné est plus foncée ou plus claire que celle des couches voisines, on dit que la lunure est rousse ou blanche. Quant à l'intensité du vice en lui-même, on peut la préjuger, d'une manière générale, d'après les circonstances suivantes:

$1^{\circ}$ Quand la couronne de bois luné est formée d'un tissu poreux, spongieux, friable, imbibé de séve noirâtre ou brunâtre, exhalant une odeur fétide, on ne peut douter que les couches qui forment la lunure ne soient déjà plus ou moins altérées, et que le bois entier de la pièce ne soit compromis dans sa qualité. Dans ce cas, la pièce entière ne peut servir à aucun usage comme bois de service, et ne peut être débitée en bois de sciage ou de fente qu'après avoir été purgée de toutes les parties viciées.

$2^{\circ}$ Quand le cercle de bois luné ne donne aucun signe d'altération, mais est formé de couches annuelles très-étroites dans lesquelles on n'aperçoit pour ainsi dire que des vaisseaux, on le considère comme un vice qui doit faire exclure la pièce des constructions, parce que la couronne de bois luné offre peu de solidité et que, par la facilité avec laquelle elle absorbe l'humidité de l'atmosphère, elle s'altèrera promptement et pourra communiquer la pourriture aux parties voisines. Les planches qui renferment du bois affecté de cette maladie sont bientôt alteintes de la vermoulure, après qu'elles sont mises en œuvre, et durent peu. Il importe donc 
d'exclure les bois lunés des planches que l'on "emploie en menuiserie, d'autant plus que, si la teinte de la lunure est blanche, elle ne laisse presque pas de trace sur le fil du bois, quand il est desséché, et que, dans le commerce, on ne fait point de différence entre ces planches et celles qui proviennent d'arbres parfaitement sains. Quant au merrain, il n'est pas de vice plus dangereux pour cette marchandise, parce qu'il est souvent difficile de le reconnấtre, quand le bois est travaillé, et qu'il suffit d'une douve de bois luné dans une futaille pour compromettre la qualité et la conservation du liquide qu'elle renferme.

$3^{\circ}$ Mais quand les couches annuelles qui forment la lunure sont aussi larges et aussi compactes qu'elles doivent l'être dans un bon bois de chêne, et qu'elles ne présentent d'ailleurs aucun signe d'altération, en un mot quand la couronne de bois luné ne diffère absolument des parties voisines que par une coloration plus claire, il est évident, à priori, que ce genre de lunure est moins immédiatement dangereux que le précédent. Les bois qui en sont atteints ne perdent rien de leur valeur commerciale lorsqu'ils sont destinés au sciage, bien qu'ils soient plus particulièrement exposés à la vermoulure, mais ils sont réputés dangereux dans l'emploi comme merrain, et on hésite toujours à s'en servir comme charpentes dans les grandes constructions, notamment dans la marine, parce que l'on n'a pas confiance dans leur solidité et surtout dans leur durée. 
Cependant, des expériences faites en Danemarck pour mesurer la ténacité des bois lunés ont prouvé que le bois dont les couches annuelles offrent une texture satisfaisante et ne diffèrent des voisines que par une coloration plus claire est plus résistant, dès qu'il est desséché, que le bon bois des couches environnantes et même que le bon bois d'arbres non lunés. "Toutefois, ajoute l'auteur (1) auquel nous D empruntons ce renseignement, dans la limite de 1) durée de ces expériences, les bois à anneaux „ blancs, ou blancs jaunâtres, absorbent plus d'eau, " quand on les immerge, que le bois qui les enve》) loppe. C'est le seul défaul qui puisse faire hésiter ") à employer les bois à anneaux blancs. Si donc on " ne met en place les bois à anneaux blancs, qu'après " les avoir complétement desséchés, s'ils sont, dans „) un vaisseau, à l'abri de l'humidité, de manière 1) que leur dessiccation continue, leur emploi offrira "beaucoup moins de danger qu'on ne pourrait le D supposer.

"Les expériences que l'on vient de rapporter ont " été faites sur des bois dont la texture el la coloration ") sont normaies et dont quelques couches annuelles 》) seulement offrent une coloration différente. Mais "le cas inverse peut se présenter, et l'on voit quel" quefois, dans du bois de qualité inférieure (bois " gras), un plus ou moins grand nombre de couches " qui possèdent tous les caractères de texture et de

(1) Mr D. H. Funch, underskibbymesler. Copenhague, 1833. 
„ coloration des meilleurs bois. Dans ce dernier cas, » il est évident, à première vue, que l'anneau ne ๖ porte aucun préjudice à la solidité de la pièce; " mais pour s'en convaincre on a soumis ces deux " natures de bois à des expériences analogues aux " précédentes, et on a trouvé que le bois de l'anneau ¿a, sous tous les rapports, des qualités supérieures "au bois qui l'environne. "

Remarquons bien que ces expériences n'ont porté que sur des bois lunés qui ne donnaient aucun signe d'altération, et n'ont eu pour objet que de mesurer la ténacité de ces bois. Loin de contester les résultats de ces expériences, nous ajouterons que l'aubier aussi, tant qu'il est encore intact, est souvent plus dur et plus tenace que le bois de cœur du chêne. Mais la ténacité n'est pas la qualité principale de cette précieuse essence, et c'est plutôt pour son élasticité et sa durée qu'elle est surtout recherchée pour les grands emplois. Or, il est constant que ni l'aubier ni le bois luné ne peuvent être comparés, sous le rapport de la durée, au bois de cœur du chêne lorsqu'il est sain; et, à défaut de sujets d'observation, on se rendra facilement compte de l'infériorité de l'aubier et du bois luné, en remarquant avec quelle facilité ce bois se dessèche et, tour à tour, s'imprègne de l'humidité atmosphérique; avec quelle avidité il absorbe l'eau dans laquelle on le plonge; combien il est poreux ; combien sa fibre est sèche et dénuée de cette substance grasse, d'apparence gommeuse ou gélatineuse, qui revêt et incruste 
le tissu des bons bois de chêne et assure leur conservation. Pour nous donc la lunure est, suivant son degré d'intensité ou d'avancement, une maladie ou un vice qui compromet les principales propriétés du chêne et qui, un peu plus tôt ou un peu plus tard, se transformera en un foyer de pourriture, longtemps avant que les couches voisines de bon bois soient alteintes de décomposition (1).

Duhamel attribuait la lunure à ce que les arbres qui en sont atteints ont crû dans des terrains maigres ou secs, ou à une maladie quelconque qui attaque les arbres et qui se guérit après un certain temps. Il a observé en outre que ce défaut se rencontre surtout dans les arbres qui ont crû aux expositions de l'Est et du Midi et, dans ce cas, il présume qu'on doit l'attribuer soit à un coup de soleil qui a desséché l'écorce et l'aubier du côté où l'arbre a été frappé, soit au verglas qui, pendant les grands froids de l'hiver, aura endommagé l'écorce et l'aubier du côté opposé au soleil.

L'auteur danois que nous citions tout à l'heure

(1) Des ouvriers fendeurs de la Franche-Comté m'ont affirmé qu'ils avaient débité en merrain les bois d'une coupe d'ensemencement dont presque tous les arbres abandonnés étaient atteints de lunare plus ou moins foncée, et qu'étant revenus sur le mème point, 9 ans après, pour débiter de la mème manière les arbres abattus dans la coupe définitive, ceux-ci s'étaient trouvés lunés comme les précédents, mais avec cette différence que la maladie avait fait de très-grands progrès depuîs l'exploitation précédente. Dans la coupe d'ensemencement, les arbres abattus étaient atteints de lunures jaunâtres on rousses dont le tissu était encore exempt de pourriture, tandis quic dans les arbres abattus, lors de la coupe définitive, les couches de bois luné étaient généralement noirâtres et en pleine décomposition. 
prétend que la lunure est produite par les couches de terre, de nature différente, que les racines de l'arbre ont dû traverser pendant son existence et, tout en remarquant que la lunure blanche est moins mauvaise que la lunure rousse, il attribue les différences de couleur de la lunure à la coloration particulière des sucs que la séve a charriés pendant le séjour des racines dans les différentes couches de terrain superposées. Au contraire, dans beaucoup de localités où cette maladie est aussi appelée gelure, on l'attribue exclusivement à des froids excessifs qui ont gelé l'aubier, en totalité ou en partie, pendant les hivers rigoureux. Quant à nous, nous n'hésitons pas à nous ranger à cette dernière opinion, d'autant plus que nos observations personnelles nous ont conduit à remarquer que la lunure se rencontre surtout dans les forêts ou dans les cantons de forêts, tels que certains bas-fonds, où les froids se font sentir d'une manière plus constante et plus intense, et que les couches de bois luné correspondent le plus ordinairement aux années d'hiver rigoureux, comme 1789 et 1830, par exemple. Tous les ouvriers fendeurs (et ce sont ceux qui connaissent le mieux les défauts du chêne) que nous avons vus travailler dans les coupes, sont aussi de cet avis et citent également les hivers de 1789 et de 1830 comme ayant produit beaucoup de lunures, notamment dans les forêts de chêne de l'Est de la France (1).

(1) On a remarqué aussi qu'une lunure située dans les couches 
Quant à la différence entre la lunure blanche et la lunure rousse, nous croyons qu'elle n'existe que temporairement, et que la couleur rousse ou brunâtre de la lunure indique tout simplement un degré plus ou moins avancé de la maladie qui, au début, affecte toujours une couleur blanchâtre ou blanche jaunâtre. Que si la couleur blanche jaunâtre de la lunure persiste plus ou moins longtemps dans un arbre que dans un autre, cela tient à la plus ou moins grande vigueur de la végétation et à la qualité du chêne qui en est atteint, les arbres les plus vigoureux et dont le bois est de la meilleure qualité résistant toujours mieux et plus longtemps aux causes de destruction quelconques dont ils sont altaqués que les chênes tendres et à végélation lente.

La lunure est toujours plus ou moins apparente sur la tranche d'un arbre fraîchement coupé, mais si le bois reste exposé à l'air et au soleil, ou s'il est seulement ressuyé et mis à l'abri de l'humidité, la teinte de la lunure se fond quelquefois avec celle des couches voisines, surtout si la lunure est blanche, de telle sorte qu'on u'en voit plus de trace. Mais, pour peu que l'on soupçonne la présence d'une lunure dans une pièce de charpente, par

voisines du cœur ne s'élève pas à une graude hauteur dans le tronc de l'arbre, et qu'un chêne peut être luné dans presque toute sa longueur, sans que le vice existe, au gros bout si l'arbre a élé coupé en terre, au petit bout si la section de la pièce a été faite près des branches audessus ou au-dessous de la naissance du houppier. Ces observations tendraient encore à confirmer l'opinion que la lunure doit être attribuée à la gelée. 
exemple, on peut la faire reparaître en versant de l'eau sur la tranche, ou mieux encore en éboutant la pièce à quelques centimètres. De même, lorsqu'on veut purger un lot ou une fourniture de merrain des pièces qui peuvent être atteintes de cette maladie, on trempe successivement chaque douve dans de l'eau, et l'on voit aussitôt, soit sur la tranche, soit sur le fil, si le bois présente des traces de lunure; on prend même souvent la précaution, dans les arsenaux de la guerre, d'arrozer les douves avec de l'eau chaude, afin de faire mieux ressortir la différence de couleur à laquelle on reconnaît les bois lunés (1). Enfin, pour le merrain, comme pour les planches ou le morceau d'une pièce de charpente éboutée, on peut encore reconnaître la lunure en mouillant la tranche du bois à un bout, et en soufflant fortement dans le sens des fibres à l'autre bout; si le bois est luné, l'air insufflé par un bout passe à travers la pièce, ce qui se reconnaît aux petits globules qui se forment à l'autre extrémité sur la tranche mouillée.

Déjà nous avons dit que l'on jugeait surtout de la qualité relative des bois de chêne d'après le développement des couches annuelles et la nature de leur tissu. Mais les chênes les plus nerveux, pas

(1) L'eau chaudes'emploie peut-être aussi dans le but de faire fondre les substances grasses, comme le suif, dont on se sert quelquefois pour enduire les couches de bois luné, el que l'eau froide ne suffirait pas à faire reparaître. 
plus que les chênes les plus tendres, ne présentent presque jamais des accroissements en diamètre bien réguliers. Ise plus souvent, au contraire, on remarque, même dans ceux qui offrent le plus d'homogénéité dans la formation de leurs accroissements successifs, des zones concentriques formées de plusieurs couches annuelles sensiblement plus étroites que celles qui précèdent et qui suivent. Quand ces zones n'affectent pas une coloration différente du reste de la tranche, et que d'ailleurs il existe une proportion convenable entre le bois d'automne et le bois de printemps de chaque couche, on ne peut pas les considérer comme un défaut. Mais si ces zones sont formées de couches tellement étroites qu'on n'y distingue plus que des vaisseaux, le bois pourra être très-accessible à la pourniture sèche et à la vermoulure, surtout s'il y a interruption complète des rayons médullaires sur la tranche formée par ces couches. Cet état constitue donc. un vice qui peut avoir la même importance que la lunure, et qui est plus difficile à observer parce qu'il n'est pas accusé par une différence de coloration bien tranchée.

S VIII. - Frollure, entr'écorce, trous de vers.

Lorsqu'une partie de l'écorce d'un chêne a été enlevée par une cause accidentelle, telle que le frottement des essieux d'une voiture, la chute d'un arbre voisin, les blanchis destinés à recevoir une 
empreinte de marteau, etc., si le liber placé à l'intérieur de l'écorce a été attaqué, il se forme toujours à l'endroit meurtri une couche de bois mort qui se recouvre d'une nouvelle écorce el que rien ne décèle à l'extérieur. C'est ce que l'on nomme une frotture $(P l . X I l, f i .1)$. Ce vice est extrêmement fréquent, mais il n'a pas toujours beaucoup de gravité, parce qu'en général il s'étend peu. Cependant si, sous la roulure partielle qui limite la frotture à l'extérieur, on s'aperçoit que le bois a une teinte verdàtre ou brunàtre différente de la couleur générale des autres couches, c'est un indice qu'il y a un commencement d'altération ou de pourriture dont il importe de purger la pièce avant de la mettre en œuvre, soit en l'entamant arec l'herminette et la gouge, soit en la tronçonnant quand la maladie a pris trop d'extension.

Les éperons ou crochets en fer dont se servent les délinquants ou les élagueurs, pour monter aux arhres, produisent des effets analogues. La piqûre du crochet dans l'écorce laisse une trace ordinairement visible à l'extérieur, et détermine à l'intérieur un épanchement de séve qui produit une tache noire plus ou moins large sur le bois et occasionne souvent la pourriture. Ce défaut, de même que la frotture dont il n'est qu'un diminutif, se manifeste surtout dans le débit du chêne en merrain, et peut être une cause de plus ou moins grand déchet dans la fabrication.

Bien que les frottures laissent toujours une trace 
sur l'écorce du chêne, elles ne sont pas toujours apparentes dans les bois abattus, aprèz qu'ils sont écorcés ou équarris; mais sourent elles se trahissent, sur l'une des faces de l'équarrissage, par des flammes de couleur foncée, ou simplement par des lignes noires ou des fentes d'éclat très-fines qu'il faut toujours visiter et sonder avec soin, quand on veut s'assurer de la gravité du vice et de la qualité du bois.

Le défaut nommé entr'écorce n'a pas non plus de gravité. Cet accident peut se présenter sur un point quelconque d'un arbre et résulte ordinairement de la soudure de deux branches entre elles; on le rencontre surtout dans les arbres fourchus dont les branches sont très-rapprochées. L'entr'écorce n'a d'autre effet que de diminuer la force de la pièce, par suite de la séparation des fibres du trone et de la branche qu'il renferme.

Les trous de vers proviennent de plusieurs espèces de larves. Ceux que produisent les gros vers du capricorne ne sont pas dangereux en ce sens qu'ils n'occasionnent pas de pourriture, mais, quand ils sont nombreux, ils peuvent avoir pour effet d'affaiblir la pièce attaquée. Ceux que produisent les petits vers sont beaucoup plus à craindre, surtout ceux qui sont dus à la larve d'un insecte appelé le Lymexilon naval. La piqûre des petits vers produit dans l'intérieur des pièces une fermentation telle que le bois 
QUALITÉS ET DÉFAUTS DES BOIS D'OEUVRE.

est bientôt grisetté et entre en décomposition. Ce sont les ravages du Lymexilon qui obligent, dans beaucoup de ports de mer, à conserver les approvisionnements de bois sous l'eau.

Lorsqu'on peut débiter à temps les pièces attaquées, en sciages par exemple, la dessiccation fait mourir les larves, arrête la décomposition du bois et le rend encore susceptible d'un bon emploi. 


\section{CHAPITRE SIXIEME.}

DE LA CONSERVATION DES BOIS D'OEUVRE.

\section{Article I.}

Des conditions dans lesquelles les bois sont employés ou mis en œuvre.

Les bois de feu, les bois de service et la plus grande partie des bois de travail ne s'emploient d'ordinaire que lorsqu'ils sont parvenus à un certain degré de desséchement.

Le bois de feu brûle mal lorsqu'il est vert, et ne donne pas, à volume égal, la même quantité de chaleur que lorsqu'il est sec, parce que, dans la combustion, il dépense une partie de sa puissance calorifique à faire évaporer l'eau qu'il renferme. C'est pourquoi on ne brûle généralement les bois de feu dans les foyers domestiques que dans l'hiver qui suit l'abatage, c'est-à-dire, après sept ou huit mois de coupe; c'est pour la même raison que dans les usines on soumet les bois de feu à une forte dessiccation avant de les employer.

Les bois de construction se dessèchent moins vite 
et moirs profondément que les bois de feu, parce qu'ils ont des dimensions plus fortes. Souvent on les emploie comme charpentes, lorsqu'ils sont encore verts ou du moins peu desséchés, pourvu que ce soit à couvert, dans un lieu sec et aéré; mais alors on évite de charger les pièces de bois vert d'un poids trop considérable parce qu'elles se courberaient et perdraient de leur force. Les longues pièces peuvent même quelquefois se conrber sous leur propre poids, de manière à compromettre la solidité d'une charpente. En général, on estime que les bois de construction ne derraient jamais être employés avant d'avoir deux ans de coupe, ceux de peuplier exceptés. Par contre, on évite aussi d'employer comme charpentes des bois extrêmement vieux et secs, parce qu'ils ont perdu une grande partie de leur élasticité et qu'ils se rompent, sans plier, sous un poids ou un effort moins considérable que s'ils étaient moins desséchés.

Parmi les bois d'industrie, il en est aussi qui ne peuvent ètre travaillés avec facilité sans se rompre, qu'à la condition de n'être pas trop desséchés. Tel est le merrain que les tonneliers sont obligés d'attendrir, lorsqu'il est sec, par l'action combinée de l'eau et du feu, pour faire prendre aux douves la courbure qu'ils veulent leur donner. Au contraire, les bois de menuiserie qui ne supportent que peu ou point de charge ne peuvent jamais être trop secs, si l'on veut éviter qu'ils ne se déjettent, se fendent et se retirent, 
lorsqu'ils sont mis en œuvre; il en est de même des bois qui doivent être employés à des ouvrages de précision.

\section{Article II.}

Des procédés ordinaires de conservation des bois d'œuvre.

Les bois de service ou de travail, quelle que soit leur destination, ne sont donc généralement travaillés et mis en œuvre qu'après un temps plus ou moins long, suivant le degré de dessiccation qu'ils doivent atteindre. Mais, pendant ce temps, les bois sont exposés à plusieurs dangers dont les principaux sont : les grosses fentes, la pourriture et la vermoulure. Pour les en préserver, on emploie des moyens différents selon les localités, et aussi selon les dangers qu'on a le plus à redouter.

Les fentes sont dues au retrait que prend le bois en se desséchant. Quoi qu'on fasse, on ne peut en préserver entièrement certains bois, particulièrement les grosses pièces de chêne, qu'en les débitant, aussitôt après l'abatage, en sciages ou en bois de fente. Quant aux bois de charpente, ce qu'on doit chercher à éviter ce ne sont pas les petites gerçures qui ne nuisent en rien à la qualité du bois, mais les grosses fentes qui pourraient diminuer la force et la solidité des pièces. Dans les pays froids et humides où l'on n'a pas beaucoup à craindre les grosses fentes, les marchands se bornent à empiler leurs bois 
en plein air, et à prendre tous les soins possibles pour les préserver de l'échauffement et de la pourriture. A cet effet, on établit sous les piles des sommiers assez forts pour que les pièces du fond ne soient pas en contact immédiat avec le sol et, dans les dépôts bien tenus, on bétonne et on pave l'emplacement des piles pour intercepter les émanations de la terre; on sépare les bois d'une même pile par des cales, de façon que l'air puisse librement circuler entre chaque pièce, et on les abrite contre la pluie et les ardeurs du soleil, en recouvrant le lit supérieur avec des planches disposées en toit. Ce moyen est le plus communément employé dans les magasins des marchands et dans les chantiers de construction de l'Etat.

Mais, dans les pays chauds, les bois ainsi empilés en plein air se fendraient et se tourmenteraient tellement, sous l'action des fortes chaleurs, qu'ils deviendraient souvent hors de service. C'est pour parer à cet inconvénient que dans le midi on abrite les bois sous des hangars bien aérés et construits de façon à préserver, autant que possible, les pièces des alternatives de sécheresse et d'humidité. On remarque, du reste, que les bois du midi, notamment les chênes de Provence, étant plus nerveux que ceux des départements du nord, sont plus disposés à se fendre, et qu'on risquerait d'en perdre un bon nombre, si l'on ne prenait tou!es les précautions nécessaires pour éviter le dessèchement brusque et inégal de ces bois. Lorsque les bois 
équarris ou débités en charpentes, traverses, etc., semblent disposés à se fendre, ce qui apparaît ordinairement dans la région du cœur, on arrête et on prévient les grosses fentes, en reliant les parties qui tendent à se séparer avec des $\mathrm{S}$ en fer que l'on enfonce dans le bois.

Ces procédés sont ceux qu'on emploie dans les arsenaux de la marine pour conserver une partie des approvisionnements en bois. Mais, quelque soin que l'on prenne, les bois empilés en plein air ou sous des hangars sont toujours exposés à la piqûre, ou bien ils s'échauffent et pourrissent sous l'influence de l'humidité et de la chaleur, ou bien, enfin, ils souffrent de l'excès de sécheresse qui nuit surtout aux bois de mâture en faisant évaporer la résine qu'ils renferment. Pour éviter ces inconvénients, on immerge les bois les plus précieux dans des réservoirs ou des bassins remplis d'eau. L'expérience a prouvé que l'eau de mer était plus favorable à la conservation des bois que l'eau douce; mais l'immersion des pièces dans l'eau de mer pure les exposerait à un ennemi bien autrement dangereux, le tarêt naval (1), qui pullule dans la mer avec une remarquable fécondité et qui s'aitache indistinctement à tous les bois. Or, on a remarqué que le tarêt ne

(1) Tarêt naval. (grandeur naturelle) (Pl. XII, fig. 2).

$a$, Mandibules tranchantes ayant la forme de tarières (en calcaire) ; $b$, petite coquille bivalve de la queue; $c$, corps du tarèt, glaireux; $d$, tubes ayant constamment un mouvement d'inspiration el d'expiration; e, logement du tarèt dans une pièce de bois. 
peut vivre dans les eaux saumâtres ni dans la vase et, suivant les localités, on s'est basé sur ce fait pour arriver à une conservation parfaite des bois de marine les plus précieux.

A Brest et dans les ports à marée, on enfouit les bois dans les vases molles qui couvrent le littoral et qui sont toujours imprégnées d'eau de mer par la marée. On a pu utiliser ainsi des mâtures du nord qui étaient enfouies dans ces vases depuis 100 ans, sans qu'on ait remarqué la moindre altération dans le bois parfait. A Toulon, on a préparé, dans le même but, d'immenses fosses dans lesquelles on introduit à la fois de l'eau de mer et de l'eau douce, jusqu'à ce que le degré marqué par le pèse-sel indique un mélange suffisant pour tuer les tarêts. C'est aussi en partie pour préserver les bàtiments des atteintes du tarêt naval, que l'on revêt d'une feuille de cuivre la face extérieure de la carène jusqu'à la hauteur de la ligne de flottaison.

Les bois que l'on façonne dans les coupes, les sciages, le merrain, etc., sont moins sujets à se fendre que les pièces de charpente en grume ou équarries; ces bois sont aussi moins exposés, surtout le merrain, à la vermoulure et à la pourriture, parce qu'ils sont ordinairement bien purgés d'aubier et de tout principe de maladie. Mais lorsque les planches et le merrain ne sont pas mis à l'abri des injures de l'air, ils se voilent et se tourmentent, si on ne prend soin de les disposer en pile de la manière que tout 
le monde connaît. Dans les magasins, les dépôts ou les chantiers, les sciages et les bois de fente s'empilent séparément et par espèce, de même que les bois ronds ou équarris, et l'on prend les mêmes précautions pour faire circuler l'air dans chaque pile.

Les planches et les charpentes du commerce sont, en général, d'autant plus recherchées qu'elles sont plus sèches. Le flottage hâte le dessèchement des bois et, pour les planches comme pour les charpentes, on remarque de plus que celles qui ont été flottées se tourmentent el se déjettent moins quand elles sont mises en œuvre. Mais le flottage altère la couleur des bois, et comme les planches sciées sur maille sont surtout employées en ébénisterie et en menuiserie à des ourrages de luxe, et que d'ailleurs elles sont moins exposées que les autres à se voiler et à se retirer par suite du dessèchement, on évite, autant qu'on le peut, de les flotter.

\section{ARticLe III.}

De la conservation des bois d'œuvre par le procédé du docteur Boucherie.

D'autres moyens sont encore employés pour conserver les bois d'œuvre. Les enduits, tels que les peintures à l'huile, le goudron, le brai, dont on a coutume de se servir, sont excellents pour empêcher que les bois ne soient pénétrés et endommagés par la pluie ou par l'humidité de l'air. Mais on ne doit en couvrir la surface entière des bois que lorsque ceux-ci sont parvenus à un certain degré de dessè- 
chement, attendu que si les enduits s'opposent à la pénétration de l'humidité extérieure, ils apportent en même temps un obstacle à l'évaporation de l'humidité renfermée dans le bois et provoquent à l'intérieur la fermentation et la pourriture.

Les autres procédés consistent à expulser la séve des bois abattus et à la remplacer par une substance qui assure leur conservation. Après bien des essais, qui furent tentés surtout en Angleterre, le docteur Boucherie, de Bordeaux, a trouvé un moyen simple de résoudre l'important problème de la pénétration des bois.

Les procédés qu'il a employés reposent sur ce principe : que le liquide qui doit donner au bois certaines propriétés particulières doit remplacer la séve qui y existe, pénétrer dans tous les espaces qu'elle occupe et l'en expulser complètement.

II. Boucherie s'était d'abord servi de la force de succion qu'exercent les feuilles pour faire pénétrer dans l'arbre encore vivant le liquide conservateur qui devait remplacer la séve. Bientôt il vit qu'une faible pression de 2 ou 3 mètres d'eau pouvait remplacer l'appel des feuilles, et qu'il suffisait que le bois fût abattu depuis peu de temps pour que le liquide conservateur pût pénétrer dans son tissu, faire écouler la séve qui s'y trouvait renfermée, en occuper la place et y déposer les matières qui doivent assurer la conservation du bois. En agissant sur des bois abattus depuis deux ou trois mois au plus 
et encore remplis de séve, on peut, sous cette faible pression, opérer la pénétration dans un temps qui varie de quelques heures à deux jours au plus, suivant la grandeur de la pièce de bois et la nature de l'arbre.

Toutes les solutions aqueuses peuvent ainsi pénétrer le tissu du bois et y déposer ensuite la matière qu'elles tenaient en dissolution; de nombreux essais ont maintenant constaté que le sulfate de cuivre était le sel qui assurait le mieux la conservation du bois placé dans les circonstances atmosphériques les plus défavorables.

Le liquide injecté pénètre seulement les tissus parcourus par la séve. Ainsi, chez beaucoup d'arbres dans lesquels l'aubier et le bois parfait forment des parties bien distinctes, comme le chêne, le mélèze, les pins, etc., l'aubier seul peut être injecté. Au contraire, les bois dans lesquels la différence entre l'aubier et le bois parfait n'est pas sensible, comme le charme, le hêtre, le sapin, l'épicéa, etc. sont pénétrés dans toute leur épaisseur. Encore observet-on que, parmi ceux-ci, toutes les parties ne sont pas également imprégnées, et c'est là, peutêtre, une des causes qui influent sur le plus ou moins d'efficacité des procédés d'injection.

Le procédé du docteur Boucherie force à opérer sur des bois de fraîche coupe, en grume, et recouverts de leur écorce. On injecte ainsi des parties qui peuvent ne pas être utilisées, puisque elles sont 
susceptibles d'être retranchées par l'équarrissage.

Pour éviter cet inconvénient, MI. Legé et FleuryPironnet ont imaginé de placer dans des cylindres en tôle les pièces équarries, travaillées, prêtes à être assemblées et mises en œuvre, et ils font arriver dans ces cylindres un jet de vapeur qui chasse la séve; puis on fait le vide et, agissant avec une pression de plusieurs atmosphères, on fait pénétrer dans l'intérieur du bois une dissolution de sulfate de cuivre chauffée à près de $70^{\circ}$. De cette manière, ils injectent tout l'aubier et obtiennent même une pénétration superficielle du bois parfait (quelques millimètres).

Quel que soit le procéde employé, nous conclurons, d'après les résultats connus d'expériences déjà anciennes, que les bois les plus exposés à la vermoulure et à la pourriture peuvent acquérir une durée beaucoup plus longue, et remplacer les bois précieux, au moins dans certains emplois, lorsqu'au préalable ils sont injectés de sulfate de cuivre. A ce point de vue, la découverte du docteur Boucherie nous paraît donc très-importante. Mais hâtons-nous de dire que si une dissolution de sulfate de cuivre, substituée à la séve el à tous les principes fermentescibles qu'elle renferme, a pour effet de retarder la décomposition et la désorganisation des tissus ligneux, cette préparation ne peut rien changer à la structure propre du bois et, par conséquent, ne doit rien ajouter à sa qualité sous le rapport de la 
ténacité et de l'élasticité (1). Que si, par exemple, une traverse de hêtre injectée de sulfate de cuivre peut remplacer, sous le rapport de la durée, une traverse de chêne, il n'est pas présumable que le hêtre, quelque préparation qu'il subisse, puisse jamais remplacer le chêne ou le pin dans les grands emplois des constructions civiles ou navales (2).

Les procédés de conservation des bois, imaginés par le docteur Boucherie et quelques autres inventeurs, consistent donc à introduire dans les tissus ligneux soit un toxique comme le sulfate de cuivre, soit des matières empyreumatiques et créosotées. Parmi ces matières, les unes, comme la créosote, sont volatiles, et leur efficacité cesse au bout de peu de temps; les autres, comme le sulfate de cuivre, sont entraînées par les eaux ou peuvent devenir

(1) Certains constructeurs sont mème portés à craindre qu'une dissolution de sulfate de cuivre, substituée à la séve, ne nuise à l'élasticité des bois de charpente en les rendant plus aptes à se dessécher complétement. Par contre, j'ai entendu exprimer l'opinion que l'on pourrait pe ut-être remédier au défaut d'élasticité du sapin, et à sa trop grande disposition à se dessécher à l'air dans certains emplois comme la mâture, si on le pénétrait d'un sel déliquescent, tel que le chlorure de cuivre ou autre, qui entretiendrait toujours une certaine bumidité dans le bois. Quant à moi, je crois que le plus grand progrès possible à faire dans celte voie serait d'arriver à utiliser l'aubier des bois de construetion, comme le chêne et le pin, en l'injectant d'une substance qui puisse suppléer à ce qui lui manque pour être aussi durable que le bois parfait.

(2) Des renseignements pris auprès des Compagnies de chemins de fer, il résulte que les traverses de hêtre les mieux préparées résistent tout au plus aussi longtemps que les traverses de chêne de qualité ordinaire. 
dangereuses quand les bois qui les contiennent sont employés dans des lieux habités. Aucun de ces procédés n'est d'ailleurs applicable à la plus précieuse de nos essences forestières, le chêne, qui est rebelle à l'injection.

Frappé de la prompte décomposition des bois de chêne, qu'une nécessité du service avait fait mettre en ouvre à bord de nos vaisseaux avant leur dessiccation, et du rapide développement de champignons qui en fut la conséquence, M. de Lapparent, directeur des constructions navales, a cherché un moyen de combattre ce danger. Dans ce but il a repris, en le perfectionnant, un ancien procédé qui consiste à carboniser la surface extérieure des bois que l'on veut mettre à l'abri de la pourriture.

Lorsqu'on carbonise le bois au feu libre, l'opération se fait inégalement, par places, et l'action du feu ne pénètre pas dans les fentes; mais si, par un moyen quelconque, on lance avec force un jet de flammes à la surface du bois, le résultat est tout différent.

Sous l'action énergique d'un jet de flamme à la température de 1,000 à 1,200 degrés, tous les germes de moisissures qui se seraient déjà déposés et accumulés à la surface du bois sont détruits, et la douche de flamme produit une légère couche charbonneuse imprégnée des matières empyreumatiques et créosotées produites par la distillation du bois sur lequel on opère. On parvient ainsi à empêcher le développement des champignons et la fermentation sur les 
faces extérieures du bois; ce procédé est, de plus, applicable à toutes les essences, et enfin les expériences faites jusqu'à présent semblent justifier pleinement les bons effets dus à son application.

C'est ainsi qu'à Cherbourg des barreaux de chêne carbonisés sur la moitié de leur longueur et enfouis pendant un an dans du fumier humide en ont été retirés parfaitement sains dans la partie carbonisée, tandis que l'autre moitié commençait à se décomposer, et que des échalas en peuplier, dont la pointe avait été carbonisée, se sont trouvés en très-bon état après un an de séjour en terre.

Actuellement, dans la plupart des ports de construction français et étrangers, on carbonise en grand toute la surface des couples et des bordages qui entrent dans la construction d'un vaisseau. L'opération se fait alors au moyen du gaz d'éclairage qu'on allume au bout d'un tuyau conducteur, traversé luimême par un second tuyau communiquant à une petite machine soufflante. Cette disposition permet de promener la flamme sur toutes les surfaces. Au besoin, on obtient le même résultat à l'aide d'une lampe d'émailleur portative alimentée par des huiles minérales. Enfin, les grandes industries emploient pour la carbonisation des traverses de chemins de fer, des poteaux télégraphiques, des perches à houblon, etc., un appareil spécial alimenté par la houille.

On a calculé que la carbonisation de la frégate cuirassée la Flandre a consommé 5,000 mètres cubes de 
gaz et que la dépense par mètre carré n'a pas dépassé 0 fr. 28 c. Le mètre cube de gaz figure dans cette évaluation au prix de $0 \mathrm{fr} .35 \mathrm{c}$., l'heure de travail à 0 fr. 25 c. ; un ouvrier carbonise 3 mètres carrés par heure. Avec la lampe d'émailleur, on a calculé que la carbonisation du mètre carré reviendrait à 0 fr. 116 (1).

(1) Cette notice et les chiffres qui l'accompagnent sont extraits d'une brochure publiée par M. de Lapparent, directeur des constructions navales. - Paris. Arthus-Bertrand. 


\section{DEUXIÈME PARTIE}

\section{ESTIMATION ET VENTE DES BOIS \\ SUR PIED.}

ESTIMATION DE LA VALEUR DES FORETS

EN FONDS ET SUPERFICIE. 



\section{ESTIMATION}

\section{ET VENTE DES BOIS SUR PIED}

\section{CHAPITRE PREMIER.}

DE L'ESTIMATION EY MATIĖRE DES BOIS SUR PIED.

\section{Article I.}

\section{Générali tés.}

L'estimation en matière des bois sur pied est une opération qui peut avoir pour objet : ou de calculer la possibilité d'une forêt, c'est-à-dire, de fixer en bloc la quotité, en mètres cubes, des produits de toute espèce qu'elle peut fournir annuellement; ou de déterminer le volume des produits de chaque espèce abandonnés à l'exploitation dans une coupe à vendre sur pied.

Une coupe est une étendue déterminée dans une forêt pour y abattre le bois en totalité, ou avec réserve d'un certain nombre d'arbres. Asseoir une coupe, ou faire l'assiette d'une coupe, c'est désigner son emplacement.

Dans les futaies, la possibilité des coupes principales s'établit par volume et s'exprime en mètres cubes. La possibilité des coupes d'amélioration se 
fonde sur la contenance et s'exprime en unités de surface. - La détermination de la possibilité par volume consiste à évaluer pour un temps limité, ordinairement une période de la révolution, le volume actuel des bois à ahattre, en coupes principales, et le volume dont ces bois s'accroîtront jusqu'au moment de leur exploitation. La somme du volume actuel et du volume futur, divisée par le nombre d'années de la période, exprime la possibilité ou le nombre de mètres cubes à exploiter chaque année, en coupes principales, pendant la durée de cette période. La recherche de la possibilité principale des futaies comprend donc deux opérations is tinctes : l'estimation du volume actuel, et le calcul du volume futur des bois à abattre dans un temps donné. Dans la pratique, ce travail se complique on outre de détails particuliers à chaque forêt et d' $\epsilon$ - périences d'accroissement qui sont plus spécialement du ressort de l'aménagement (1). Nous n'aborderons pas ici l'examen des procédés dont on se sert pour calculer l'accroissement futur des bois; n? nous bornerons simplement à dire, dans le cours de, ce chapitre, comment on procède à l'estimation du volume actuel des bois à comprendre dans le calcul de la possibilité des futaies, et comment on doit appliquer cette possibilité dans l'assiette et l'estimation des coupes annuelles à vendre sur pied.

Dans les taillis, la possibilité des coupes princi-

(1) Voir notre Cour's d'aménagement. 
psles s'établit par contenance. Elle s'exprime en unités de surface et s'obtient en divisant l'étendue totale de la série par le nombre d'années de la révolution. Chacune des coupes à exploiter successivement a, par conséquent, la même étendue, et ses limites sont fixées à l'avance sur le terrain; mais les produits de chaque coupe ne sont pas assujettis, comme dans les futaies, à un rendement déterminé.

Chaque année, les agents forestiers, chefs de service, dressent un état des coupes à exploiter dans leur circonscription pour l'exercice suivant, quelquefois même pour deux exercices. Ce tableau indique la nature des exploitations à faire dans chaque coupe, la quotité en mètres cubes des produits à fournir par les coupes dont la possibilité est basée le volıme, et l'étendue que l'on veut donner à celles qui s'exploitent par contenance. - Ce tableau, auquel on donne le nom d'Etat d'assiette des coripes, est soumis à l'examen et à l'approbation de l'Administration centrale, qui contrôle et quelquefois modifie les propositions qu'il renferme, et le renvoie ensuite aux Agents chargés de la direction des opéracions forestières.

En terme de métier, on comprend sous le nom d'opérations de martelage celles qui ont pour objet la désignation des arbres à réserver ou à exploiter dans les coupes, et l'estimation en matière des bois à vendre sur pied.

Dans les coupes de taillis, les arbres ou baliveaux de toutes catégories que l'on veut réserver sont 
marqués, à la racine ou au pied, du marteau de l'Etat, savoir : les baliveaux de l'âge et les anciens, d'une seule empreinte; les modernes, de deux empreintes sur deux blanchis; cela s'appelle marteler, marquer ou baliver en réserve. L'estimation en matière des bois abandonnés à l'exploitation se fait en même temps que le balivage, ou immédiatement après.

Dans les coupes de futaie, on balive tantôt en réserve, comme dans les taillis, et tantòt en délivrance. Le balivage en délivrance consiste à marquer sur deux blanchis, l'un au corps et l'autre à la racine, les arbres qui doivent être exploités. Soit que l'on marque en réserve ou en délivrance, on procède en même temps à l'estimation des arbres abandonnés, afin de pouvoir arrêter le martelage et les limites de la coupe aussitôt que le volume des arbres désignés pour être abattus atteint le chiffre de la possibilité.

Les principaux procédés qui sont employés dans la pratique des estimations sont au nombre de quatre, savoir :

L'estimation par le comptage et le cubage individuel des arbres;

L'estimation à vue d'œil et par pied d'arbre;

L'estimation à vue d'œil, par hectare ou par rirée ;

L'estimation par places d'essai. 
ARticle II.

De l'estimation par le comptage et le cubage individuel des arbres.

\section{SI. - Exposé de la méthode.}

Ce mode d'estimation trouve son application la plus importante, et un emploi à peu près exclusif, dans le calcul de la possibilité par volume des coupes principales de futaie. Dans ce cas, étant connue la surface à parcourir par ces coupes dans un temps déterminé, on dénornbre tous les arbres à exploiter en mesurant le diamètre de chacun d'eux, et on les classe par catégories, dont chacune renferme tous ceux qui ont sensiblement la même hauteur et dont les diamètres sont compris entre des limites assez rapprochées, $0^{\mathrm{m}}, 20$ à $0^{\mathrm{m}}, 25,0^{\mathrm{m}}, 25$ à $0^{\mathrm{m}}, 30$, par exemple; on détermine par des expériences le volume moyen, ou volume type, d'un arbre pour chaque classe de diamètre, et on multiplie ce volume type par le nombre d'arbres appartenant à la même catégorie. Faisant ensuite la somme de tous ces produits, on obtient le volume actuel total des bois à abattre en coupes principales dans un temps donné et, quand on ne veut pas tenir compte du volume futur dans le calcul de la possibilité annuelle, on la déduit immédiatement de ce matériel total en le divisant par le nombre d'années qui devra être employé à le réaliser. 
Cela posé, nous allons voir comment on peut procéder au mesurage des dimensions de la tige des arbres, au calcul du volume réel des arbres types et, subsidiairement, aux expériences qu'il est toujours utile de faire à l'occasion de la recherche de la possibilité des futaies.

\section{\$II. - Mesurage des dimensions de la tige des arbres.}

Les dimensions que nous avons besoin de connaître sont, avons-nous dit, la grosseur et la hauteur des arbres. La grosseur s'exprime par la circonférence ou le diamètre mesuré à hauteur d'appui, $1 \mathrm{~m}$. ou $1^{\mathrm{m}}, 33 \mathrm{du}$ sol ou, pour mieux dire, à l'endroit où la tige commence à prendre sa forme régulière. La mesure de la circonférence est plus rigoureuse que celle du diamètre, mais elle est plus difficile à prendre exactement, et l'usage a généralement prévalu, surtout dans les comptages qui ont pour objet la détermination de la possibilité des futaies, de mesurer les diamètres avec le compas forestier.

Pour mesurer exactement la circonférence, on enroule le ruban gradué autour de l'arbre, de manière à figurer une section perpendiculaire à l'axe. A cet effet, on fixe avec une pointe l'extrémité ou le zéro du ruban sur l'écorce, puis on le déroule et on l'applique sur la tige en faisant le tour de l'arbre.

Le mesurage des diamètres avec le compas forestier est plus expéditif, mais pour faire un emploi convenable de cet instrument, il y a plusieurs pré- 
cautions à prendre. D'abord il faut s'assurer de sa bonne confection, en ce qui concerne l'exacte graduation de la grande règle et le jeu régulier de la règle mobile. Il faut en outre, dans le maniement de l'instrument, que la grande règle soit toujours appuyée contre le corps de l'arbre et perpendiculairement à son axe. L'instrument ainsi placé, on fait mouvoir la règle mobile de manière à serrer légèrement le corps de l'arbre entre les deux petites règles; on lit alors une première fois la mesure du diamètre sur la grande règle, puis une seconde et une troisième fois, en présentant l'instrument sur toutes les faces de l'arbre, et on prend la moyenne entre ces différentes mesures pour la grosseur ou le diamètre de l'arbre à sa base. La grande règle étant divisée en centimètres, le mesurage des diamètres avec le compas forestier peut se faire de centimètre en centimètre, ou de deux en deux, ou de cinq en cinq centimètres, selon le degré d'approximation que l'on recherche. Mais lorsqu'on opère de cinq en cinq centimètres, il est bon, pour éviter les erreurs de lecture, de faire correspondre les chiffres 10,15 , 20, 25, etc., aux graduations $71 / 2,121 / 2,17$ 1/2, $221 / 2$ centimètres de la grande règle. Cette précaution est surtout utile lorsqu'on opère sur une grande échelle, par exemple dans les comptages de possibilité, et que l'on est obligé de confier l'opération à des ouvriers ou à des subordonnés peu intelligents ou peu soigneux. On prend d'ailleurs, contre ces ouvriers, toutes les mesures nécessaires 
pour assurer la fidélité et la bonne exécution de leurs opérations.

Jusqu'à présent, nous ne nous sommes occupés que du comptage des arbres et du mesurage des diamètres. Or, il est bien clair qu'avant de les classer d'après leurs diamètres, pour appliquer le même volume type à tous'ceux d'une même catégorie, il est nécessaire de tenir compte de leur hauteur. On comprend, en effet, que suivant les terrains et les autres conditions de la végétation, les arbres de même essence et de même diamètre peuvent avoir des hauteurs très-inégales et, par conséquent, des volumes bien différents. Si donc on tient à calculer la possibilité avec quelque approximation, il faut bien tenir compte de cette circonstance et, pour un mème diamètre, faire des catégories séparées et calculer un volume type distinct pour les parties ou parcelles d'une même forêt, dont les arbres présenteraient des différences sensibles sous le rapport de la hauteur. De là la nécessité, en certain cas, de rechercher la hauteur moyenne des arbres d'une même catégorie.

Le moyen le plus sûr et le plus simple de l'obtenir, c'est de choisir quelques arbres qui, à simple vue, paraissent avoir la hauteur moyenne de tous ceux de la mêrne classe, de les faire abattre, et d'en mesurer la longueur quand ils sont par terre. Mais ce moyen n'est pas toujours praticable et, dans bien des cas d'ailleurs, il est bor de savoir mesurer la hauteur d'arbres sur pied. On se sert, à cet effet, d'instruments appelés dendromètres, au moyen des- 
quels on peut obtenir, sans calcul, la hauteur d'un arbre ou d'une partie d'arbre en fonction d'une longueur mesurée horizontalement sur le sol. On en a imaginé de très-ingénieux, mais qui ont l'inconvénient d'être trop compliqués ou peu portatifs. Nous ne nous occuperons que des plus simples, parce qu'ils sont aussi les meilleurs.

La planchette ordinaire, ou dendromètre-planchette, est un rectangle aux deux extrémités duquel sont appliquées, sur l'épaisseur des petits côtés, deux petites réglettes en cuivre (Planche $I$, fig. 14). Ces régleties débordent le côté supérieur du rectangle, de façon à présenter une ligne le mire déterminée par un trou 0 formant oculaire à l'une des réglettes, et par une fenêtre que traverse un fil horizontal $F$ formant objectif à l'autre. L'arête inférieure $\mathrm{AB}$ de la planchette est divisée en centimètres et en millimètres à partir du point $\mathrm{A}$; elle est parallèle à la ligne de mire $\mathrm{OF}$, et distante de cette ligne d'une longueur fixe égale à $0^{\mathrm{m}}, 10$. Le fil horizontal qui fait objectif porte un plomb P. L'instrument se tient à la main, à l'aide d'un anneau fixé au centre de la planchette du côté opposé à la graduation $\mathrm{AB}$.

Voici comment on fait usage de la planchette :

On se transporte à une distance horizontale de 10 mètres de l'arbre que l'on veut mesurer; on place la ligne de mire $\mathrm{OF}$ dans la direction du rayon visuel aboutissant au point $\mathrm{C}$ dont on veut avoir la hauteur. Dans cette position, le fil à plomb FP vient battre le côté inférieur de la planchette, et forme un triangle 
rectangle FAK, semblable au triangle OCD formé dans l'espace par la longueur CD, l'horizontale $\mathrm{OD}$ et la ligne de visée OC. Le côté FA est l'homologue de OD et le côté AK l'homologue de CD. Mais OD $=10$ mètres et $\mathrm{FA}=0^{\mathrm{m}}, 10$, c'est-à-dire que $\mathrm{OD}$ $=\mathrm{FA} \times 100$; done, $\mathrm{CD}=\mathrm{AK} \times 100$. Ce qui revient à dire que le nombre de centimètres et de millimètres marqués par le fil à plomb de $\mathrm{A}$ en $\mathrm{K}$, exprimera en mètres et en décimètres la hauteur $\mathrm{CD}$, à laquelle on ajoutera, la hauteur DE que l'on mesurera directement pour aroir la hauteur cherchée CE.

La planchette est un instrument très-commode et qui donne la hauteur des arbres avec l'approximation désirable. Mais elle exige que l'on stationne à 10 mètres, ou bien à 20 mètres en doublant la longueur $\mathrm{AK}$ interceptée par le fil à plomb, ou bien à une distance quelconque, auquel cas on n'obtient plus la hauteur directement, mais par un calcul de proportion.

On évite ces inconrénients en se servant du dendromètre à base variable de $\mathrm{II}$ Regneault $(P l .1$, fig. 15).

Cet instrument est formé de deux règles ajustées à angle droit et divisées toutes deux en centimètres et millimètres. L'une A B supporte les pinnules, l'autre $\mathrm{CD}$ porte le fil à plomb; cette dernière glisse à volonté dans une rainure, en restant perpendiculaire à $\mathrm{AB}$, ce qui permet de donner à $\mathrm{OC}$ une longueur exactement égale au centième de la distance 
du pied de l'arbre au point de station et, par conséquent, de choisir à volonté ce point de station. On se sert, du reste, de ce dendromètre comme on se sert de la planchette.

En prolongeant suffisamment la règle $\mathrm{A} 0$ de 0 en $B$, ce dendromètre peut servir à mesurer sans déplacement la hauteur $\mathrm{H}^{\prime}$, en lisant la graduation interceptée par le fil à plomb de 0 en $B$.

Pour opérer avec l'un ou l'autre de ces instruments, il faut être deux: l'un des opérateurs met l'instrument en station, l'autre lit les graduations. Mais on peut éviter cet inconvénient en se servant de la planchette à perpendicule de M. Bouvart, inspecteur des forêts.

La construction de cet instrument $(P l .1, f g .6)$ est fondée sur les propriétés des tangentes.

Deux planchettes rectangulaires sont juxta-posées de manière à laisser entre elles un espace libre dans lequel se meut, autour d'un axe placé en $\mathrm{A}$, un perpendicule terminé par un are gradué; la graduation est double et symétriquement tracée des deux côtés de la ligne de foi du perpendicule. Une ouverture pratiquée dans le bas de la planchette laisse apercevoir la graduation, et un index fixe coïncide avec le zéro lorsque la ligne de visée est horizontale. Un ressort muni d'un bouton $\mathrm{R}$ donne ou ôte la liberté au perpendicule.

Lorsqu'on se sert de ce dendromètre et qu'on vise le haut de l'arbre au moyen des pinnules placées en $\mathrm{V}$ et $\mathrm{V}^{\prime}$, le perpendicule, obéissant aux lois de la pe- 
santeur, maintient sa ligne de foi dans la verticale, et l'arc que l'index mesure indique l'angle d'inclinaison du rayon visuel. Si la graduation était établie en parties aliquotes du cercle, comme celle des éclimètres, il faudrait, pour avoir la hauteur, multiplier la distance horizontale qui sépare l'observateur de l'arbre par la tangente de l'angle d'inclinaison, et par conséquent se servir de tables; mais les divisions du limbe étant celles qui correspondent aux angles dont les tangentes, pour le rayon 1 , sont 0,01 , $0,02,0,03,0,04$, etc., etc., il suffira de multiplier la distance horizontale par le nombre qu'indiquera l'index pour avoir la hauteur cherchée.

Voici comment on se sert de l'instrument : on se place en un point d'où l'on puisse apercevoir à la fois le pied de l'arbre et le point de la tige dont on veut connaître la hauteur; on vise successivement ces deux extrémités par les pinnules $\mathrm{V}$ et $\mathrm{V}^{\prime}$, on donne la liberté au perpendicule en pressant le bouton $\mathrm{R}$ pendant la visée, et quand le perpendicule est redevenu immobile, on le fixe dans la position qu'il a prise en lâchant le bouton. On lit chaque fois la graduation qui se trouve sous l'index, on fait la somme des deux cotes lues et on multiplie la distance horizontale par cette somme; le produit donne la hauteur cherchée à partir du sol.

Pour éviter les multiplications, on pourrait placer sur le revers de la planchette une petite table où on les trouverait toutes faites. 
\III. - Détermination du volume réel des arbres types.

Les arbres ayant été dénombrés et classés par catégories de diamètres, on en fait abattre un certain nombre choisis, dans chaque catégorie, parmi ceux qui paraissent offrir la forme et la hauteur qui se rapprochent le plus de la moyenne des arbres de même essence. Considérant un de ces arbres en particulier, on décompose la tige en billes de un ou de deux mètres de longueur, jusqu'à ce qu'on arrive à la partie supérieure qui, eu égard à sa forme, peut être regardée comme un cône. On cube chacun des billons comme un cylindre de même longueur et de grosseur égale au diamètre mesuré en son milieu. On cube séparément l'extrémité supérieure de la tige comme un cône régulier. On fait la somme des volumes de tous les cylindres et du cône terminal, et l'on obtient avec une très-grande approximation le volume réel de la tige.

Pour obtenir le volume des branches, on distingue dans chaque catégorie les arbres très-branchus, moyennement branchus, et peu branchus; on établit ainsi trois classes dans chaque catégorie ; on fait débiter et façonner séparément toutes leurs branches en bois de corde et en fagots. On cube exactement les bûches de chauffage et les fagots, par les procédés que nous avons décrits pages 42 et suivantes, et on obtient ainsi, pour chaque arbre d'expérience, le volume réel de tout le bois compris dans les branches. 
La somme du volume réel de la tige et de celui des branches représente le volumé réel de l'arbre sur lequel on opère. En répétant cette expérience sur un certain nombre d'arbres de chaque catégorie, on en déduit des moyénnes qui représentent le volume de l'arbre type pour chaque classe de diamètres.

Quand on procède aux calculs et aux différentes opérations que nous venons d'indiquer, pour la détermination du volume réel des arbres types, il est bon de recueillir ces données avec un certain ordre, et de les consigner dans un calepin spécial d'expériences, dont nous donnons un modèle aux pages 238 et 239 .

Lorsqu'on fait de ces expériences, soit à propos de la détermination de la possibilité des futaies, soit en vue de l'estimation en matière des arbres à abattre dans les coupes annuelles, on s'enquiert naturellement de la destination et de l'emploi des produits de chaque forêt. Dès lors, on est conduit à rechercher, par catégorie d'arbres, le rendement du mètre cube brut de bois de travail en marchandises fabriquées, et le rapport du volume brut du bois de service au volume des mêmes pièces débitées selon les usages du commerce de la localité. Ces nouveaux coefficients sont très-importants à connaître par les Agents chargés d'exécuter les aménagements et de procéder à l'estimation en matière et en argent des produits à vendre dans les coupes. En effet, on sait que, dans la fabrication du merrain par exemple, 
à volume égal deux pièces de bois de même qualité, mais de dimensions différentes, fourniront des produits inégaux, et que le rendement de la plus forte en diamètre sera plus avantageux que celui de la plus faible. On sait aussi, que, à volume égal, le rendement en sciages du chêne et du sapin varie avec la forme et les dimensions des pièces; que si, par exemple, un chêne tortueux ou de petite dimension ne peut fournir, avec beaucoup de déchet, qu'une petite quantité de planches, ou du sciage de médiocre qualité, souvent il est possible d'en tirer un parti plus avantageux en le débitant en bois de charronnage ou en traverses de chemin de fer, etc. On sait encore que l'équarrissage et le débit des bois de construction entraînent un déchet plus ou moins considérable selon la destination des pièces; que, pendant que la marine ne reçoit que des bois équarris à vive arête ou à peu près, les bois de commerce ne s'équarrissent souvent qu'au quart sans déduction; qu'enfin, la destination et par suite la valeur en argent de ces bois dépendent le plus souvent de leurs dimensions en diamètre et en longueur. 


\section{CALEPIN D'EXPÉRIENCES.}

Parcelle ou canton A. - Chêne de 190 ans.

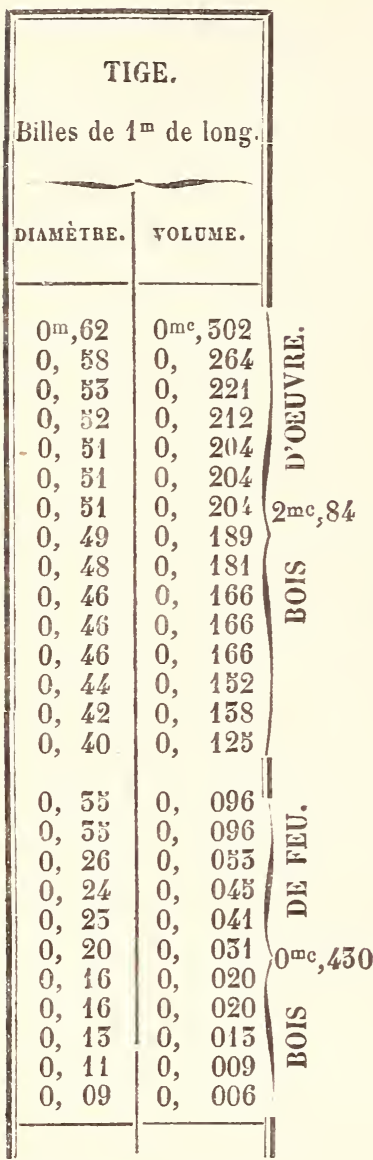

\section{BRANCHES.}

Billes de $1^{\mathrm{m}}$ de longueur.

\begin{tabular}{|c|c|c|}
\hline DIAUĖTKE. & NOMBRE. & TOLUME. \\
\hline $0^{\mathrm{m}}, 06$ & 28 & $0^{\mathrm{mc}}, 078$ \\
\hline 0,08 & 17 & $0, \quad 08$ อ \\
\hline 0,10 & 13 & $0, \quad 103$ \\
\hline 0,12 & 11 & $0, \quad 124$ \\
\hline 0,14 & 3 & $0, \quad 046$ \\
\hline 0,16 & 2 & $0, \quad 040$ \\
\hline 0,20 & 2 & $0, \quad 063$ \\
\hline 0,22 & 1 & $0, \quad 058$ \\
\hline 0,24 & 1 & $0, \quad 040$ \\
\hline 0,26 & 2 & $0, \quad 106$ \\
\hline TотаL... & 80 & $0^{m}, 728$ \\
\hline
\end{tabular}

Les branches débitées et faconnées en bois de feu ont donné 1 st., כ00 de bois de corde et 18 fagots.

Le volume réel du bois com. pris dans un cent de fagots (d'après expériences), étant de $1^{\text {mo }}, 200$, le volume de 18 fagots équivaut à $0^{\mathrm{mc}}, 2: 16$, d'où le volume total des branches $=0^{\mathrm{mc}}, 944$.

$0^{\text {" }}, 08$ base idu cône

$5^{\mathrm{mi}}$ hauteur terminal. . $0^{\mathrm{mc}}, 005$

Volume total de la tige. $\overline{5^{\mathrm{mc}}, \mathbf{3 2 9}}$ 
De ces chiffres on déduit facilement les renseignements ciaprès, renseignements au moyen desquels il est souvent possible de déterminer pour une même forêt des lois dans la végétation, et des cuefficients qui peuvent être très-utiles à consulter pour le travail des estimations en matière et en argent des coupes à vendre sur pied.

Diamètre de la tige à $1^{\mathrm{m}}, 33 \mathrm{du} \mathrm{sol} \mathrm{.} \mathrm{.} \mathrm{.} \mathrm{.} \mathrm{.} \mathrm{.} 0^{\mathrm{m}}, 58$

Hauteur totale de l'arbre. . . . . . 29 $9^{\mathrm{m}}, 00$

Volume réel de la tige. . . . . $3^{\mathrm{mc}}, 329$

Volume du bois d'œurre de la tige. . . . . 2, 894

Volume réel du bois de feu de la tige.... . 0,435

Volume réel du bois de feu des branches.. . . 0, 944

Volume total de l'arbre, tige et branches.. . . 4, 273

Volume conique de la tige........ 2, 5 รั4

Facteur du volume des branches par rapport au volume réel de la tige. . . . . . .

Proportion du bois d'œurre compris dans le volume réel de la tige........ . 87 p. $0 / 0$

Proportion du bois d'œurre compris dans le volume total de l'arbre . . . . . . 67 p. $0 / 0$

Proportion du bois de feu compris dans le volume total de l'arbre.......... . 33 p. $0 / 0$

Facteur de conversion du volume conique au volume réel de la tige ......... . . 1,30

Le diamètre de la tige (bois d'œuvre) va en décroissant de $0^{\text {m }}, 012$ par mètre de hauteur.

Le diamètre au milieu de la tronce du bois d'œuvre égale 0,84 du diamètre de base.

Le dianètre au petit bout de la tronce du bois d'œuvre égale 0,69 du diamètre de base. 
\$IV. - Estimation des arbres abandonnés à l'exploilation dans les coupes de futaie ou de taillis composé.

Le procédé que nous venons de décrire est le seul, nous le répétons, qu'il convienne d'appliquer à la détermination de la possibilité des futaies, parce qu'il est le seul dont les résultats puissent être sérieusement contrôlés et vérifiés. On s'en sert également pour déterminer le volume brut des arbres à abattre dans les coupes de futaie et des réserves abandonnées à l'exploitation dans les coupes de taillis composé.

Et d'abord, on comprend que dans les futaies dont la possibilité principale a été calculée d'après ce mode d'estimation, on comprend, disons-nous, que pour faire une application rationnelle et exacte de cette possibilité, on doive nécessairement employer le même procédé à l'estimation en matière des coupes annuelles. Cela est évident; mais, dans tous les cas possibles, il ne s'agit pas seulement d'appliquer la possibilité matérielle dont le chiffre est indiqué par l'état d'assiette, il faut encore estimer la valeur en argent des produits qui seront abandonnés à l'exploitation et, par conséquent, déterminer, avec toute l'exactitude possible, les quantités de bois d'œuvre et de bois feu que la coupe pourra fournir.

A cet effet, les gardes estimateurs mesurent et appellent le diamètre à la base de chacun des arbres à abattre, ainsi que la hauteur de la partie de la tige 
propre à donner du bois d'œurre. Quant aux branches et aux arbres qui ne peuvent être débités qu'en bois de chauffage, on les estime séparément soit à vue d'œil, soit d'après les résultats d'expériences faites sur des arbres abattus. Avec ces données et les coefficients d'expérience qui ont dû être préalablement déterminés, on calcule avec toute l'exactitude désirable :

$1^{\circ}$ Le volume brut des bois à abattre dans chaque coupe, conformément aux prescriptions de l'état d'assiette ;

$2^{\circ}$ La proportion du bois d'œuvre et du bois de feu compris dans le volume entier de la coupe.

Le premier de ces calculs s'exécute sur le terrain, parce que les Agents qui opèrent doivent s'assurer, sur le terrain même, que le produit des arbres abandonnés atteint et ne dépasse pas le chiffre de la possibilité. Le second peut ne s'effectuer que plus tard, lorsque les Agents auront à rédiger le procès-verbal d'estimation en argent des produits à vendre sur pied. Et comme le bois d'œurre se décompose dans une certaine proportion en bois de service et de travail de qualité et de valeur variables, les Agents prennent note sur le terrain de l'emploi et de la destination probable des produits, selon les dimensions des arbres et la qualité des bois, selon la situation de la coupe, la facilité ou les difficultés de la vidange, enfin et surtout selon la nature des marchandises qui, dans le moment, sont plus particulièrement recherchées par le commerce. Ils apprécient 
en même temps la proportion des produits divers en bois de feu, et ils tiennent compte de tous les frais qu'exigeront l'exploitation et le façonnage des bois, le débit ou la transformation en marchandises fabriquées de chaque espèce, et le transport au lieu de consommation. Munis de tous ces renseignements et des facteurs qui expriment le rendement du mètre cube en marchandises fabriquées, les Agents peurent calculer, avec toute l'approximation voulue, la valeur en argent des bois à vendre sur pied dans chaque coupe.

L'estimation en matière des réserves abandonnćes à l'exploitation dans une coupe de taillis composé peut se faire de la même manière. Mais comme les produits bruts de ces coupes ne sont pas assujettis à un rendement déterminé d'avance, on se borne ordinairement à estimer le volume du bois d'œurre de chaque arbre considéré individuellement, d'après ses dimensions en diamètre et en hauteur, et à évaluer en stères de chauffage et en fagots le volume du houppier. Ici, comme dans les coupes de futaie, on obtient le volume du bois d'œuvre en considérant cette partie de la tige comme un cylindre, dont la circonférence moyenne ou le diamètre moyen est égal à la circonférence ou au diamètre de la base diminué d'une certaine quantité. La loi de décroissement du diamètre ou de la circonférence se détermine par des expériences sur des arbres abattus et s'exprime à tant de centimètres ou de millimètres 
par mètre de hauteur. Assez généralement dans les taillis, on admet que la circonférence au milieu d'une pièce de bois d'œurre, essence chêne, est égale aux $9 / 10$ de la circonférence à $1^{\mathrm{m}}, 33 \mathrm{du}$ sol. Mais comme la forme de la tige des arbres varie souvent beaucoup, de forêt en forêt, ou même de canton à canton dans une même forêt, il est prudent de vérifier cette loi sur les arbres abaltus dans les coupes en exploitation, avant de l'appliquer à l'estimation des arbres à abattre dans les coupes voisines. Quant à la hauteur, ordinairement peu considérable de cette partic de la tige, on la déterminera toujours facilement et avec une exactitude suffisante, par comparaison avec les divisions d'une perche de trois ou quatre mètres de longueur que l'on appliquera verticalement contre le corps de l'arbre. Le volume du houppier, qui n'est propre qu'à donner du chauffage, s'estime le plus souvent à vue d'œil, à cause des différences considérables que présente le développement de la tête des réserves dans les taillis et des difficultés qu'il y aurait à déterminer, d'une manière satisfaisante, le rapport existant entre le volume des branches et le volume de la tige des arbres de chaque catégorie.

Pour effectuer les calculs de cubage de la partie de la tige propre à donner du bois d'œuvre, nous venons de dire que l'on assimilait cette partie de l'arbre à un cylindre de même hauteur et de circonférence égale à la circonférence du milieu de la pièce. 
Cette pratique se fonde sur l'usage établi dans le commerce de cuber les bois en grume abattus, comme des cylindres de même longueur et de circonférence égale ou à la circonférence du milieu ou à une moyenne proportionnelle entre les circonférences mesurées au petit bout et au gros bout. Or, il est constant que la différence entre les circonférences au gros bout et au milieu d'une pièce de bois d'œurre, est comprise entre 0 et $35 \mathrm{p} .0 / 0$ au plus de la circonférence au gros bout; autrement dit, que si la circonférence au gros bout est égale à $\mathrm{C}$, la circonférence du milieu est égale à $\mathrm{C}$, si la pièce est cylindrique, ou approximativement égale à $0,9 \breve{s} \times \mathrm{C}$, $0,90 \times \mathrm{C}, 0,85 \times \mathrm{C}, 0,80 \times \mathrm{C}, 0,75 \times \mathrm{C}, 0,70 \times \mathrm{C}$, $0,65 \times \mathrm{C}$, selon que la pièce s'approche ou s'éloigne davantage de la forme cylindrique.

En se fondant sur cette donnée d'expérience, on pourrait construire un tarif qui, pour une circonférence de base et une hauteur quelconques, donnerait les volumes cylindriques correspondant à la même hauteur et à la même base diminuée de $\tilde{5}, 10$, $15,20,25,30$, ou 35 p. 0/0. Pour appliquer ce tarif à l'estimation d'arbres sur pied, il suffirait de mesurer la circonférence de base et la hauteur de la partie de la tige propre au bois d'œuvre, puis de déterminer, par quelques expériences sur des arbres abattus, le rapport moyen de la circonférence du milieu à la circonférence de base et, par suite, la colonne du tarif dans laquelle on devra trouver le volume de la pièce à cuber. Ce tarif pourrait être établi de la manière suivante : 
DES BOIS SUR PIED.

\begin{tabular}{|c|c|c|c|c|c|c|c|c|c|}
\hline \multirow{2}{*}{\multicolumn{2}{|c|}{ 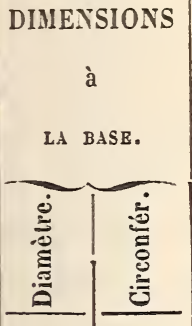 }} & \multicolumn{8}{|c|}{$\begin{array}{l}\text { VOLUMES CYLINDRIQUES } \\
\text { nètre de hauteur et pour des dimensions } \\
\text { es à celles de la base diminuées de : }\end{array}$} \\
\hline & & 0,00 & 0,05 & 0,10 & 0,15 & 0,20 & 0,25 & 0 & 5 \\
\hline $\begin{array}{c}\mathrm{m} . \\
\mathbf{0}, 20\end{array}$ & $\begin{array}{c}\mathrm{m} . \\
\mathbf{0 , 6 3}\end{array}$ & $\begin{array}{l}\text { m.c. } \\
0,051\end{array}$ & \begin{tabular}{l|} 
m.c. \\
0,028
\end{tabular} & m.c. & $\begin{array}{l}\text { m.c. } \\
0,0,23\end{array}$ & $\begin{array}{l}\text { m.c. } \\
\text { o,020 }\end{array}$ & $\begin{array}{l}\text { m.c. } \\
0,018\end{array}$ & $\mid \begin{array}{l}\text { m.c. } \\
0,015\end{array}$ & $\begin{array}{l}\text { m.c. } \\
0,015\end{array}$ \\
\hline 25 & 0,88 & 0,049 & 0,044 & & $0,0 \beth 5$ & 0,051 & 0,028 & & 0,02 \\
\hline 0,30 & 0,94 & 0,071 & 0,064 & & 0,031 & 0,045 & 0,040 & & 0,03 \\
\hline 85 & 1,10 & 0,096 & 0 , & 0,078 & 69 & 0,062 & & & 0,04 \\
\hline (n) & $\mathbf{1}, \mathbf{2 6}$ & 0,126 & 0,113 & & 0,091 & 0,080 & 0,071 & & 0,05 \\
\hline 0,45 & $1, \mathbb{1}$ & 0,159 & 0,143 & & 0,115 & 0,102 & & & 0,067 \\
\hline 0.50 & 1,58 & 0,196 & 0,177 & 0,159 & 0,142 & $0,126 \mid$ & & & 0,08 \\
\hline $0, \mathbf{5 5}$ & 1,83 & 0,258 & 14 & 0,192 & 0,172 & 0,152 & & & 0,10 \\
\hline 80 & 1,88 & 0,283 & $0,2 \%$ & $0,229 \mid$ & 0,204 & 0,181 & & 58 & 1. \\
\hline 35 & 2,04 & 32 & 0,299 & 0,269 & 0,240 & 0,212 & 87 & & 0,14 \\
\hline & 2,20 & & 0,347 & 0,512 & 78 & 6 & 0,216 & & 0,1 \\
\hline & $\mathbf{2}, \mathbf{3 6}$ & 0,442 & $0, \mathbf{5} 98$ & $0,3 \Xi 8$ & 19 & 0,283 & 0,248 & & 0,18 \\
\hline & $\mathbf{2}, \mathbf{5} 1$ & 5 & $0,4 \mathrm{n} 4$ & 0,407 & 65 & 22 & & & 212 \\
\hline & $\mathbf{Z}, 68$ & 0,567 & 2 & 460 & 0,410 & & & & 0,240 \\
\hline & $\mathbf{2}, \mathbf{8 3}$ & 0,636 & 0,374 & $0, \check{1}$ & 0,460 & & & & 0,26 \\
\hline & $\mathbf{2}, 98$ & 0,709 & 0,640 & $0,57 \dot{4}$ & 12 & 0,40 & 0,598 & & 0,29 \\
\hline & 3,14 & 0,785 & 0,709 & 0,636 & & $0,305 \overline{-}$ & 0,442 & 85 & 0,00 \\
\hline,- 00 & $\mathbf{3}, \mathbf{3 0}$ & 0,866 & 0 & 1 & 25 & 34 & 0,487 & $0,424 \mid$ & 0 \\
\hline $\mathbf{1}, \mathbf{1 0}$ & $3,4=5$ & 950 & 0,858 & 0,770 & 0,686 & 0,608 & 0,534 & 0,466 & 0,40 \\
\hline $\mathbf{1}, \mathbf{1 5}$ & 3,61 & 1,0579 & 0, & 0,841 & 0,7 ว 0 & 0,665 & 0,384 & 0,509 & 0,43 \\
\hline $\mathbf{1}, \mathbf{2 0}$ & 3,86 & 1,151 & 1,021 & $0,916 \mid$ & 0,817 & 0,724 & 0,656 & 0,554 & 0, \\
\hline 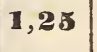 & $\mathbf{3}$, & 1,227 & 1,107 & 0,994 & 0,886 & 0,785 & 0,690 & 0,601 & \\
\hline & 4, & 327 & 1,198 & 1,075 & 0,959 & 0,849 & 0,746 & 0,650 & 0 , \\
\hline
\end{tabular}


Aptricle III.

Estimation à vue d'œil et par pied d'arbre.

Le mode d'estimation à vue d'œil et par pied d'arbre s'applique, comme le précédent, aux arbres de réserve abandonnés à l'cxploitation dans les coupes de taillis composé, aux arbres à exploiter en coupes principales dans les futaies régulières, et au $\mathrm{x}$ arbres à exploiter en jardinant dans les futaies irrégulières. Son emploi consiste à estimer en stères le volume de chaque arbre, tige et branches, comme s'il devait être converti tout entier en bois de feu. Puis, quand l'opération est faite et qu'il s'agit de fixer la valeur en argent de la coupe, on détermine la proportion en bois d'œurre et en bois de chauffage du matériel estimé soit d'après des notes prises sur le terrain, soit d'après des renseignements recueillis dans des coupes précédemment exploitées, soit d'après les résultats d'expériences faites sur des arbres abattus.

Ce procédé est en usage dans beaucoup de forêts, et particulièrement dans les futaies dont tous les produits sont destinés au chauffage. Mais quand les arbres peuvent donner du bois d'œurre, les règlements administratifs prescrivent aux agents estimateurs de mesurer et d'inscrire sur leur calepin la circonférence de base et la hauteur de la partie de la tige qui pourra être convertie en bois de service 
ou d'industrie. Or, à supposer que cette prescription n'implique, pour l'Administration, qu'un moyen de contrôler les opérations des agents estimateurs, on comprend cependant que cette mesure les porte à se contrôler eux-mêmes, en vérifiant par le calcul l'estimation du bois d'œuvre qu'ils ont pu faire à vue d'œil. Ce procédé tend donc, sinon à disparaître de la pratique des estimations, du moins à se modifier de façon à se confondre, ou à peu près, avec le mode par comptage et cubage individuel des arbres.

L'art d'estimer à vue d'œil et par pied d'arbre ne peut pas s'enseigner, mais il est assez facile à acquérir; aussi rencontre-t-on, dans toutes les localités forestières, bon nombre d'estimateurs habiles en ce genre. Ces hommes, exercés par une longue pratique des exploitations, jugent du volume d'un arbre en le comparant par la pensée à d'autres arbres de même essence, de mêmes dimensions et de même forme dont ils connaissent le rendement, parce qu'ils les ont vu exploiter et débiter. Quelle que soit l'habileté du forestier qui s'en sert, ce mode d'estimation, on le conçoit, ne peut jamais conduire à des résultats bien certains. Souvent les erreurs sont peu importantes, mais quelquefois aussi elles sont considérables; de là, des inconvénients qui ont plus ou moins de gravité selon les circonstances dans lesquelles on opère. Au point de vue de la vente des produits, une estimation erronée du ma- 
tériel à exploiter conduit à une évaluation en argent trop faible ou trop forte des produits à vendre sur pied, ce qui est toujours fâcheux. Au point de vue économique, ce mode d'estimation appliqué aux coupes annuelles dans les futaies aménagées présente des dangers plus sérieux, en ce sens que l'on peut être amené à anticiper sur l'avenir, ce qui forcerait plus tard à suspendre les exploitations, ou au moins à réduire considérablement la possibilité des produits restant à exploiter.

Mais, de ce que nous n'approuvons pas l'emploi exclusif de ce procédé dans l'estimation des coupes de futaie, est-ce à dire que nous regardons comme superflu pour un forestier de savoir estimer à vue d'œil, avec une certaine approximation, le volume d'un arbre sur pied et son rendement en marchandises fabriquées? Loin de là. Nous considérons, au contraire, que tout forestier, pour devenir un praticien habile et consommé, doit s'efforcer de devenir bon estimateur; que non-seulement il doit apprendre à estimer les arbres individuellement, mais qu'il doit encore s'appliquer à estimer approximativement, par hectare, des massifs entiers de bois de tous âges et de toute consistance. Pour arriver à ce résultat, il faut se former le coup d'œil en observant beaucoup, en suivant de près les exploitations, en faisant abattre, façonner et débiter sous ses yeux des arbres que l'on a vus sur pied, que l'on a examinés sous tous les aspects, et dont on s'est fixé les formes et les dimensions dans la mémoire. Ou bien, il faut 
suivre assez longtemps et très-attentivement les opérations d'un estimateur habile, et vérifier les types que l'on s'est créés, ou se rectifier le coup d'œil par des expériences sur les arbres abattus ou à abattre dans les coupes en exploitation. Le premier pas à faire dans cette étude et le plus important, c'est de s'exercer à estimer juste le diamètre et la hauteur d'un arbre quelconque, et on y arrive assez vite en s'aidant, dès le début, du compas forestier et du dendromètre. Ces premières données acquises, on se perfectionne d'autant plus vite que l'on fait plus d'applications, et que l'on se donne la peine de se vérifier par le procédé plus sûr que nous avons exposé dans l'Article second. Disons seulement pour finir que, lorsqu'on veut estimer à vue d'œil un arbre sur pied, il faut d'abord l'approcher de tout près, pour se rendre bien compte de son diamètre à hauteur d'homme et de la manière dont la tige se soutient; qu'ensuite, il faut l'examiner de plus loin et sous tous les aspects, de manière à bien voir tout le développement des branches et de la cime. Après ce premier examen, on estime séparément la partie de la tige propre à donner du bois d'œuvre en stères ou en mètres cubes, et le restant de l'arbre en stères de chauffage et en fagots. 
Article IV.

Estimation à vue d'œil, par hectare ou par virée.

L'art d'estimer les bois sur pied à vue d'œil et en masse ne peut être enseigné, parce qu'il est impossible de donner à cet égard aucune règle théorique précise. Ce mode d'estimation ne s'emploie en général que pour déterminer le volume sur pied d'une masse de bois de faibles dimensions et qui, eu égard à la multiplicité des tiges, ne pourraient être soumis à un mode d'estimation plus rigoureux, sans entraîner une perte de temps considérable. Telles sont les coupes de taillis qui s'exploilent à de courtes révolutions, et dont tous les produits doivant être convertis en bois de chauffage. Dans ce cas, l'estimateur parcourt la coupe plusieurs fois et dans tous les sens, et après s'être bien rendu compte de la nature et de la consistance des peuplements, de l'étendue relative des vides et des massifs les plus complets, par rapport au peuplement moyen, de la hauteur et de la grosseur des tiges, et enfin de la manière dont les bois sont plantés, il évalue approximativement le volume du bois à abattre et son rendement en stères de chauffage ou de charbonnette et en fagots. Cette évaluation se fait le plus souvent par unité de surface, par hectare, pour deux raisons : d'abord, parce qu'autrefois les coupes de cette nature se vendaient à l'hectare dans les forêts de l'Etat et des 
communes ; parce qu'aussi ce mode de vente est encore en usage dans la plupart des taillis possédés par les particuliers, et que vendeur's et acheteurs ont dù s'habituer à estimer de cette manière; en second lieu, ces coupes s'exploitant par contenance, on a été conduit à rapporter à l'unité de surface le rendement des coupes exploitées, et à se créer ainsi, pour chaque espèce de peuplement, des types de production qui devaient servir à estimer sur pied les autres coupes à exploiter les années suivantes.

C'est dans la possession de ces types et l'art de les appliquer à l'estimation de peuplements semblables que gît tout le talent de l'estimateur. C'est assez dire que ce mode d'estimation exige de celui qui l'emploie une grande rectitude de coup d'œil, du jugement, de l'esprit d'observation et de comparaison, et surtout une longue pratique des exploitations. Quant au moyen d'acquérir ce talent si précieux pour un forestier, il consiste à beaucoup observer, à examiner de très-près les peuplements des coupes à exploiter, à assister à l'abatage, à la découpe, au façonnage et au mesuragge des bois, et à se rendre exactement compte des produits fournis par les peuplements dont on s'est fixél'image dans la mémoire.

Dans les contrées où les produits des taillis peuvent recevoir différentes destinations, selon les essences qui entrent en mélange dans les peuplements et selon les dimensions des perches, l'estimation du bois sur pied se fait quelquefois à vue d'œil et par 
virée. Ainsi, un taillis dont les produits seront façonnés, partie en bois de feu, partie en bois d'industrie tels que échalas, perches à houblon, étançons de mine ou de houillère, etc., ou qui se vend avec faculté d'écorcer quand il renferme du chêne, un semblable taillis s'estimera quelquefois à vue d'œil et par hectare, si l'on a des termes de comparaison assez sûrs, mais plus souvent en détail et par virée. Dans l'un et l'autre cas, l'estimateur apprécie directement la quantité de marchandises de chaque espèce que le taillis doit fournir, par hectare si l'estimation a lieu à vue d'œil et par hectare, par virée si l'estimation a lieu à vue d'œil et par virée. Toutefois, lorsqu'on opère par virées dont on ne connaît pas la contenance, il vaut mieux évaluer en détail et successivement la quantité de chaque espèce de marchandises que renferme la partie du peuplement qu'on veut estimer.

Ce mode par virée consiste à diviser la surface de la coupe en bandes étroites et parallèles que l'estimateur parcourt successivement, en se tenant à égale distance des lignes qui servent de limites à la bande dont il estime les produits.

Nous avons dit que l'art d'estimer les masses à vue d'œil consiste dans l'application exacte des types de production que l'expérience a gravés dans la mémoire de l'estimateur. Or, il est un fait qui influe beaucoup sur le rendement des taillis et dont nous voulons prévenir ceux qui n'ont pas encore une habi- 
tude suffisante des estimations; c'est, en termes de métier, la manière dont les bois sont plantés. - Par la manière dont les bois sont plantés dans les taillis, on exprime si le peuplement est surtout formé de cepées plus ou moins fortes, ou s'il y a mélange de petites cepées, de brins de semence et de drageons. Un taillis qui se compose surtout de fortes cepées est heaucoup plus productif qu'un taillis aussi complet en apparence, mais dont le peuplement est formé en grande partie de brins de semence ou de drageons. Comme conséquence de ce fait, les taillis d'aune, de chêne, de frêne, de châtaignier, de tilleul, dont les souches produisent beaucoup de rejets, sont généralement plus productifs que les taillis d'essences qui poussent peu de rejets, et se reproduisent surtout par drageons ou par brins de semence.

Donc, en résumé, lorsqu'on veut faire l'estimation d'une coupe de taillis, soit par hectare, soit par virée, il importe tout d'abord de se rendre compte de la proportion du mélange des essences, de la hauteur et de la grosseur des tiges, et de la manière dont les bois sont plantés. Ce n'est qu'après cet aperçu général que l'on peut rapporter et comparer le peuplement à estimer aux différents types de production des taillis simples, ou du sous-bois dans les taillis composés. 
Article V.

De l'estimation en matière des bois sur pied, par places d'essai.

Le mode d'estimation par places d'essai consiste à estimer à vue d'œil ou, plus généralement, à cuber aussi exactement que possible, par les procédés de la dendrométrie, le matériel existant sur une surface déterminée, et à appliquer les résultats de cette estimation aux massifs situés dans les mêmes conditions de peuplement. L'emploi de ce mode exige évidemment que la place d'essai dont le matériel servira de type dans le calcul, soit déterminée de façon qu'elle soit bien l'expression moyenne du peuplement entier. Si le massif à estimer présente des nuances tranchées dans la consistance du peuplement, la forme et les dimensions des arbres, on doit établir autant de places d'essai différentes et donner à chacune d'elles une contenance proportionnelle à l'étendue de la masse correspondante du massif à estimer, ou bien, appliquer séparément à chaque masse de peuplement différent les résultats de la place d'essai correspondante.

Ce qui frappe d'abord dans l'emploi de ce mode d'estimation, c'est qu'il ne peut être appliqué qu'à des masses assez considérables et d'un peuplement à peu près uniforme. En effet, pour une coupe de petite étendue, il serait aussi expéditif de l'estimer tout entière en détail, surtout si l'on considère que, pour 
opérer avec chance de succès, il faut toujours donner une certaine étendue aux places d'essai et en établir un certain nombre, afin d'avoir des résultats moyens suffisamment exacis. Si ensuite on réfléchit au mode d'exécution de l'opération, il se présente au début une difficulté grave, c'est l'établissement même des places d'essai. Il arrive en effet que, quelque soin que l'on apporte dans le choix de ces places, on n'est jamais certain de prendre bien exactement ses types et, par suite, on est contraint de douter soimême des résultats qu'ils donneront. Or, une erreur commise, soit dans le choix du peuplement type, soit dans le résultat de son estimation, peut devenir très-grave, lorsqu'on applique ce résultat à l'estimation d'une masse considérable. Aussi ce procédé n'est-il nullement employé dans l'estimation des coupes de futaie, et si parfois on s'en sert dans les taillis, ce n'est que lorsque la coupe à estimer est très-régulièrement peuplée, et que l'on manque des données nécessaires pour l'estimer à vue d'œil par comparaison avec d'autres coupes précédemment exploitées.

\section{ARticle VI.}

De l'estimation des bois d'œuvre sur pied en produits façonnés.

Nous venons d'exposer les différentes manières d'estimer sur pied le volume brut des bois d'œuvre, le volume brut et fabriqué des bois de feu. Ces pro- 
cédés sont ceux dont les agents forestiers se servent généralement dans l'estimation des coupes à vendre sur pied. Mais, dans beaucoup de localités, tous les marchands de bois et même aussi quelques agents forestiers procèdent autrement. Ainsi, dans les sapinières, il est d'usage assez général parmi les marchands d'estimer chaque arbre individuellement, d'après ses dimensions, en marchandises fabriquées. Par exemple, un sapin de telle ou telle dimension est estimé comme pouvant donner une charpente de telle ou telle catégorie, soit un chevron, une panne simple, une panne double ou une poutre; tel autre qui est propre au sciage est estimé comme devant donner un nombre déterminé de planches réduites ou marchandes. Cette manière d'estimer une coupe de sapins suffit souvent au marchand pour déterminer le prix qu'il peut en offrir, parce qu'en beaucoup de circonstances les remanants d'exploitation et le bois de feu provenant, soit de la cime des arbres vifs, soit de la tige de quelques arbres morts, n'ont pour lui qu'une valeur insignifiante.

Dans les forêts de bois feuillus, les marchands estiment souvent les bois d'œurre, et particulièrement les chênes, en solives ou en décistères, par l'application immédiate des formules d'équarrissage. Ils se servent, à cet effet, de tarifs qui donnent les volumes au 1/4 sans déduction, au $1 / 6^{e}$ ou au $1 / 5^{e}$ déduit des pièces dont ils ont mesuré la hauteur et la circonférence de base. Le choix du mode de cubage qu'ils emploient dépend, en général, des usages 
adoptés dans la localité pour la vente des bois abattus.

Ce mode d'estimation du bois d'œuvre ne donne pas le volume réel, mais seulement le volume du bois supposé façonné. Il en résulte que les agent forestiers ne peuvent l'employer que comme un moyen de se rendre compte des opérations des marchands et de déterminer la valeur en argent des bois d'œuvre, conformément aux usages de la localité. L'emploi de ce procédé ne les dispense pas d'estimer le volume entier et brut des bois d'ouvre, d'abord parce que l'Administration tient à connaître la production réelle de chaque coupe, en second lieu parce que dans les coupes de futaie dont la possibilité est basée sur le volume, les Agents doivent pouvoir justifier que, dans le martelage de chaque coupe, ils sont restés dans les limites de cette possibilité. 


\section{CHAPITRE DEUXIÈME.}

DE L'ESTIMATION EN ARGENT DES BOIS A VENDRE SUR PIED.

Le balivage et l'estimation des coupes à vendre sur pied dans les forêts de l'Etat, des communes et des établissements publics sont confiés aux Agents chargés de l'administration de ces forêts. L'article 78 de l'ordonnance rendue pour l'exécution du Code forestier dit : "qu’il sera procédé à chaque opéra" tion de balivage et de martelage par deux Agents " au moins. "L'article 81 de la même ordonnance ajoute "que l'estimation des coupes sera faite par " un procès-verbal séparé. " Enfin, les instructions de l'Administration portent que " les estimations " des coupes marquées seront faites en commun par " les Agents qui ont procédé au martelage, et ne " doivent être connues que d'eux seuls. "

La circulaire du 9 mai 1840 traçait la marche à suivre dans l'estimation des coupes annuelles, mais une autre circulaire du 26 mai 1848 donne aux agents forestiers toute latitude sur le choix du mode d'estimation en matière. Les agents forestiers sont donc libres d'adopter tel mode d'estimation qui leur convient; mais l'Administration exige que les détails de l'opération soient consignés dans des calepins 
spéciaux qu'ils doivent conserver avec soin, pour pouvoir les présenter à tout fonctionnaire chargé de vérifier leur service. Ces calepins renferment l'indication de tous les renseignements à fournir sur le mode d'exécution et sur les résultats des opérations de balivage et d'estimation de chaque coupe.

En ce qui concerne l'estimation en matière des produits, ces indications diffèrent avec la nature de la coupe, mais on doit toujours faire connaitre :

$1^{\circ}$ Le nombre d'arbres de futaie abandonnés à l'exploitation avec la mesure, pour chacun d'eux, de la hauteur et de la circonférence prise à 1 mètre du sol ;

$2^{\circ}$ Le nombre de mètres cubes de bois de service et de bois d'industrie de chaque qualité;

$3^{\circ}$ Le nombre de stères de bois de chauffage de diverses qualités ;

$4^{\circ}$ Le nombre de stères de bois à charbon;

$5^{\circ}$ Le nombre de bottes d'écorce;

$6^{\circ}$ Le nombre de fagots et de bourrées;

$7^{\circ}$ Le volume total des arbres de la coupe d'après les tarifs d'aménagement.

Le calepin d'estimation doit renfermer en outre le détail des frais qu'entraînera l'exploitation de chaque coupe, savoir :

$1^{\circ}$ Facteur, serment et marteau;

$2^{\circ}$ Abatage des arbres de futaie;

$3^{\circ}$ Elagage des arbres;

$4^{\circ}$ Chauffage du garde X... (façon et transport);

$5^{\circ}$ Bois de chauffage (façon); 
$6^{\circ}$ Bois à charbon (façon);

$7^{\circ}$ Ecorces (façon);

$8^{\circ}$ Fagots (façon).

On complète ces renseignements par la consignation sur le calepin d'observations particulières sur le débit des bois, sur l'emploi et la destination probables des produits, sur le mode de vente dans la localité, sur les difficultés plus ou moins grandes que présente la vidange des produits de chaque coupe et sur les voies et moyens que le propriétaire met à la disposition de l'adjudicataire pour faciliter la sortie, le transport et le débit des bois.

Ces documents suffisent pour établir la valeur brute de chaque unité de marchandise dont la vente a lieu ordinairement sur le parterre de la coupe. Mais lorsque les produits se vendent exclusivement sur un marché, un port ou un lieu de dépôt déterminé, et n'ont cours qu'après y avoir été transportés, il est nécessaire de tenir compte des frais de transport et de les ajouter aux frais d'abatage et de façon.

C'est avec ces données, prises en forêt, que l'on rédige le procès-verbal d'estimation en argent de la valeur des produits à vendre sur pied dans chaque coupe. Des imprimés spéciaux sont fournis aux Agents pour la rédaction de ces actes. Ils se divisent en deux parties :

La première est relative à l'estimation en matière des produits et comprend :

$1^{\circ}$ Le nombre d'arbres de futaie abandonnés à l'exploitation; 
$2^{\circ}$ Le classement ou la décomposition du volume total en bois de service, d'industrie, de chauffage et de charbon;

$3^{\circ}$ Le nombre de fagots, de bourrées et de bottes d'écorce.

La seconde partie a rapport à l'estimation en argent des produits matériels divisés en bois de service, d'industrie, de chauffage, etc., de différentes qualités. L'application du prix de vente à la quantité de chaque espèce et qualité de produits donne au total la valeur brute de la coupe. On obtient ensuite sa valeur nette sur pied en déduisant de l'estimation brute :

$1^{\circ}$ Le bénéfice de l'adjudicataire à raison de..... p. $0 / 0$ de l'estimation brute;

$2^{\circ}$ Le traitement du facteur ou garde-vente ainsi que les frais de serment et marteau;

$3^{\circ}$ Les frais d'exploitation (élagage, abatage et façon) calculés d'après les notes consignées dans le calepin d'estimation;

$4^{\circ}$ Les travaux mis en charge ;

5o Pour les forêts domaniales :

Les délivrances aux usagers (valeur du bois);

Le chauffage du garde (transport et valeur du bois);

Pour les forêts communales:

Les droits fixes de timbre et d'enregistrement des actes de toute nature concernant la coupe.

Reste.... 
262 de L'estimation en ARGENT DES BOIS SUR PIED.

Déduisant encore :

$41 / 2$ p. 0/0 de ce reste pour droits d'enregistrement et frais d'adjudication dans les forêts domaniales,

$21 / 2$ p. $0 / 0$ de ce reste pour taux approximatif des droits d'enregistrement et de caution dans les forêts communales,

La différence exprime la valeur nette de la coupe. 


\section{CHAPITRE TROISIEMIE.}

DES DIFFÉRENTES OPÉRATIONS A FAIRE DANS LES COUPES, SELON LE MODE D'ADJODIGATION ET D'EXPLOITATION DES PRODUITS.

\section{Article I.}

De la vente des bois sur pied et des opérations préparatoires qu'elle exige.

On a vu au chapitre précédent que les ventes de bois sur pied, dans les forêts soumises au régime forestier, doivent être précédées d'opérations qui exigent la coopération de deux agents forestiers. $\mathrm{Ce}$ sont, pour chaque coupe, le martelage des arbres réservés ou des arbres abandonnés à l'exploitation, et l'estimation en matière et en argent de tous les bois à exploiter.

Nous avons décrit les procédés en usage dans la pratique des estimations; nous avons apprécié le mérite de ces procédés et nous avons indiqué les circonstances dans lesquelles chacun d'eux trouvait son application; enfin nous avons fait connaître la nature des données à recueillir pour arriver à une appréciation exacte de la valeur des produits, et nous avons énuméré tous les détails à comprendre dans la rédaction du procès-verbal d'estimation de chaque coupe. 
Quant à l'opération du balivage et du martelage, elle consiste, avons-nous dit, à choisir et à marquer les arbres à réserver ou à abattre dans les exploitations. Le choix et la marque des arbres à réserver ou des arbres à abandonner se font en même temps ; c'est pourquoi l'on confond ordinairement les deux opérations en une seule, et l'on comprend sous l'une ou l'autre dénomination : le balivage qui consiste à faire choix des arbres à réserver ou à exploiter, et le martelage qui consiste à imprimer une marque, ordinairement celle du marteau de l'Etat, sur les arbres choisis et désignés par le balivage.

De toutes les opérations de détail qui sont commandées par la culture et l'exploitation des bois, il n'en est pas qui soit plus importante que celle du balivage; il n'en est pas qui exige plus de soin, de précaution, de réflexion et de savoir faire de la part d'un agent forestier; car, soit que l'on opère dans les futaies ou dans les taillis sous futaie, soit que l'on fasse une coupe de régénération ou d'amélioration, le succès de l'opération ou le résultat qu'on en attend dépend tout entier et exclusivement de la manière dont le balivage est effectué. Aussi, ne peuton qu'approuver les dispositions de la loi qui veut "qu'il soit procédé à chaque opération de balivage par deux Agents au moins, "surtout si l'on se place au point de vue spécial du sylviculteur, et si l'on veut voir dans cette prescription un moyen de garantir la bonne exécution des opérations de culture. 
Les procès-verbaux de balivage et de martelage servent à la rédaction de l'affiche des ventes. Ils servent aussi à établir le nombre d'arbres réservés, ou le nombre de souches exploitées que l'adjudicataire doit représenter au récolement de sa coupe. Les procès-verbaux d'estimation déterminent les prix auxquels peuvent être adjugées les coupes qu'ils concernent.

Le mode d'exploitation, le mode de vidange des produits et le mode de paiement du prix principal de la coupe et des frais d'adjudication sont déterminés par le cahier des charges générales relatives à la vente des coupes annuelles, et par un cahier de clauses spéciales applicables aux exploitations de chaque arrondissement forestier.

\section{ARTICLE II.}

De la rente des bois faconnés par économie ou par entreprise, et du mode d'exploitation de ces produits.

Les opérations dont nous venons de parler s'appliquent plus particulièrement aux coupes dont les produits doivent être vendus sur pied et ensuite abattus, façonnés et utilisés au gré de l'adjudicataire ou de l'acheteur. Mais, dans le traitement des forêts, il est des exploitations qui ne peuvent être bien faites, au point de vue de la culture, qu'autant que l'on procède graduellement à la désignation des arbres à abattre, à mesure que l'on a constaté l'effet 
produit sur la consistance du peuplement par la chute des premiers arbres abattus. Telles sont, en général, les coupes d'amélioration que l'on désigne sous le nom d'éclaircies et de nettoiements. - Tous les arbres à abattre dans ces coupes ne pouvant être désignés avant l'exploitation, sans compromettre le succès ou la bonne exécution de l'opération, ne peuvent, par conséquent, être estimés et vendus sur pied comme les bois à exploiter dans une coupe de taillis ou dans une coupe principale de futaie. D'où la nécessité d'adopter un autre mode d'exploitation et de vente de ces coupes. Celui qui fut le plus généralement appliqué depuis une trentaine d'années, consiste à faire exploiter les bois sous la direction des agents forestiers, soit par des ouvriers payés à la tâche ou à la journée, soit par un entrepreneur responsable qui se charge d'exécuter l'abatage et le façonnage à des prix arrêtés par adjudication publique au rabais. Après le façonnage, les produits sont dénombrés par le chef du cantonnement el ensuite vendus, par adjudication publique, en détail et par lots, soit sur le parterre des coupes, soit dans l'une des communes les plus voisines.

De ces deux modes d'exploitation, le dernier (mode d'exploitation par entreprise) est beaucoup plus usité que le premier (mode par économie), et s'applique non-seulement aux coupes d'amélioration, mais quelquefois aussi aux coupes principales des forêts de l'Etat qui restent invendues, et aux coupes de 
toute espèce dans certaines forêts communales et d'Etablissements publics.

Les avantages de ce mode d'exploitation sont les suivants :

Dans les coupes d'amélioration, éclaircies et nettoiements, la désignation des arbres à abattre n'a lieu qu'au fur et à mesure de l'exploitation, ce qui permet de mieux espacer les tiges réservées et de mieux distinguer celles qu'il est utile ou nécessaire d'abattre ou de conserver. - A cet effet, l'agent ou le préposé forestier qui dirige l'exploitation fait une première désignation, par un simple griffage, des arbres qui doivent nécessairement tomber, puis, après l'abatage de ces arbres, il revient une seconde et une troisième fois sur le même point, pour marquer ceux des arbres restants à comprendre dans l'exploitation. Dans une coupe d'éclaircie, par exemple, le premier griffage atteindra tous les bois morts ou dépérissants, le second portera sur les brins dominés ou sur le point de l'être, et le troisième désignera les tiges bienvenantes, mais surabondantes, qu'il importe de faire disparaittre pour desserrer convenablement le massif. De même, dans une coupe de nettoiement, le premier griffage portera sur les bois dominants qui nuisent le plus par leur couvert et qui sont tout à fait inutiles au soutien des bois durs; le second atteindra les sujets qui ne sont pas encore nuisibles par leur couvert, mais qui le deviendront bientôt, et qui ne sont pas d'ailleurs nécessaires pour maintenir le massif à l'état com- 
plet; le troisième portera sur les arbres qui nuisent par leur couvert, mais dont la présence est plus ou moins nécessaire pour conserver le massif complet, ou pour servir d'appui aux tiges de bois dur; les arbres atteints par ce dernier griffage seront désignés les uns pour être abattus ou ébranchés, les autres pour être étêtés (1).

Le mode d'exploitation par entreprise appliqué aux coupes principales de futaie ou de taillis sous futaie permettrait également de mieux choisir et espacer les arbres à réserver dans ces coupes; mais, d'ordinaire, celles qui s'exploitent par entreprise sont martelées à l'avance comme les coupes à vendre sur pied. Par conséquent, le seul avantage qu'on peut trouver à les exploiter de cette façon ne peut résider que dans la vente en détail des produits façonnés.

Dans les localités où ce mode d'exploitation est installé depuis plusieurs années, dans certaines forêts communales notamment, la vente en détail des produits façonnés, des bois de feu surtout, donne habituellement de bons résuliats et fait profiter le propriétaire de tout le bénéfice qu'il faut abandonner à l'adjudicataire dans les ventes de bois sur pied. Mais, pour les forêts qui, comme celles de l'Etat, sont susceptibles de fournir annuellement des produits variés et considérables, ce mode d'exploitation

(1) Il est bien entendu que chaque opération d'éclaircie ou de nettoiement ne nécessite pas toujours absolument le même nombre de griffages. 
et de vente, tel qu'on l'a appliqué jusqu'à présent, c'est-à-dire, partiellement et seulement dans les coupes d'amélioration, peut présenter des inconvénients dont les principaux sont :

$1^{\circ} \mathrm{D}$ 'exiger, pour les frais d'abatage et de façonnage, une dépense considérable et hors de proportion avec les ressources mises à la disposition de l'Administration pour cet objet. - D'où résulte souvent la nécessité d'ajourner l'exécution de certaines coupes dont l'exploitation est urgente, ou bien de les marteler et de les vendre sur pied, ce qui peut avoir des inconvénients plus ou moins graves selon la nature de l'opération et l'état des peuplements ;

$2^{\circ}$ De ne permettre de débiter les bois qu'en un petit nombre d'espèces de marchandises d'un usage général, sans avoir égard aux besoins particuliers et quelquefois individuels qui peuvent se présenter. Ce qui peut avoir pour effet de ne pas tirer tout le parti utile et commercial des produits;

$3^{\circ} \mathrm{D}$ 'avilir le prix des bois, quand la marchandise est abondante, les consommateurs spéculant sur la vente forcée de produits qui ne peuvent rester longtemps sur le parterre des coupes sans se détériorer ;

$4^{\circ} \mathrm{D}$ 'établir entre l'Administration et les marchands de bois une concurrence fâcheuse, à la suite de laquelle les produits de toute nature éprouvent toujours une dépréciation plus ou moins considérable. 
Article III.

De la vente des bois sur pied à tant l'unité de produits façonnés

Pour éviter les inconvénients que présentent le mode d'exploitation par entreprise et la vente en détail des produits façonnés, des agents forestiers ont proposé et l'Administration a autorisé un nouveau mode d'exploitation et de vente des coupes d'amélioration qui consiste à vendre les bois sur pied, soit au rabais, soit à l'enchère, à des prix déterminés pour chaque unité des différentes sortes de marchandises dans lesquelles les bois à exploiter pourront être débités.

Voici le mécanisme de ce mode d'adjudication :

L'unité de la marchandise qui a le cours le plus régulier dans la localité (le stère de hêtre, chauffage, par exemple) étant prise pour type, on compare à sa valeur moyenne la valeur moyenne de toutes les espèces de marchandises qu'on suppose pouvoir être confectionnées avec les bois dont l'exploitation doit avoir lieu. Le résultat de ces diverses comparaisons donne une série de facteurs dont chacun multiplié par la valeur de l'unité type, exprime la valeur correspondante de l'unité qu'il représente. Le tableau de ces facteurs (ils peuvent varier pour chaque coupe), est annexé aux affiches annonçant la vente, et l'adjudication se fait au rabais ou à l'enchère, pour chaque coupe, sur la mise à prix de l'unité 
prise pour type. En réalité et par suite du mécanisme des facteurs, la mise à prix et l'adjudication portent sur tous les produits de la coupe à la fois, car le prix auquel l'adjudication est tranchée pour l'un d'eux est un des éléments servant à la détermination de la valeur de vente de chacun des autres.

Comme dans les coupes par entreprise, l'exploitation est précédée d'un ou de plusieurs griffages, mais elle se divise en deux opérations distinctes, l'abatage et le faconnage.

Pour assurer la bonne exécution de l'opération et pour éviter des inconvénients ou des abus dont la nature n'a pas besoin d'être expliquée, l'abatage se fait sous la direction des agents forestiers, par des ouvriers choisis par eux et payés soit par l'Administration, soit par l'adjudicataire des produits. Cette opération donne lieu à une dépense peu considérable. - Le façonnage du bois ne commence qu'après que l'abatage est entièrement terminé. Il a lieu aux frais de l'adjudicataire, qui a toute latitude pour transformer les produits en l'espèce de marchandise qu'il désire, pourvu qu'elle soit comprise dans l'état des facteurs et que ses dimensions soient strictement conformes à celles indiquées dans le cahier des charges. Ces restrictions sont indispensables soit pour arriver à la fixation du prix de la coupe, soit pour conserver aux facteurs une valeur qu'ils n'ont obtenue que par une comparaison dans laquelle les dimensions entraient comme un des principaux éléments. En outre, dans un but d'utilité 
publique facile à comprendre, les portions d'arbres propres à donner du bois d'œuvre, et qui sont préalablement désignées par l'agent forestier, doivent rester en grume, au moins jusqu'après le dénombrement des produits, quand bien même il serait plus avantageux pour l'adjudicataire de les débiter en bois de feu.

Le façonnage terminé, il est procédé à un dénombrement contradictoire qui fixe la quantité de chaque espèce de marchandise. Le procès-verbal de cette opération contient en outre l'application à ces quantités du prix résultant de l'adjudication. Le total forme alors ce qu'on appelle le prix principal de l'adjudication, lequel sert à l'évaluation des frais proportionnels prévus par le cahier des charges.

Ce nouveau mode d'exploitation et de vente des coupes d'amélioration participe de tous les avantages du précédent, puisque la même latitude est laissée aux agents forestiers en ce qui concerne la désignation des arbres à abattre. De plus, le nouveau système évite les défauts reprochés au mode par entreprise, car il procure une grande économie dans les dépenses spéciales de l'Administration, il permet de proportionner le débit des diverses natures de marchandises, aux besoins de la consommation, il rend la vente facultative de forcée qu'elle était, et supprime la concurrence par laquelle le prix des bois pouvait être avili.

Toutefois, comme toute méthode nouvelle, celleci présente dans son application certaines difficultés 
que la pratique seule fera successivement disparầtre, au moins pour la plupart.

Ainsi l'Administration fait abaltre tous les bois avant de délivrer le permis de façonner à l'adjudicataire, d'où il résulte : $1^{\circ}$ que le parterre de la coupe peut être embarrassé au point de rendre difficile l'abatage en plusieurs fois; $2^{\circ}$ que le façonnage est retardé, ce qui peut être une cause de dépréciation pour cerlains produits qui demandent à être façonnés et vidés dans un court délai; $3^{\circ}$ qu'en abattant les bois pêle-mêle, les tiges peuvent se briser en tombant les unes sur les autres. - Le façonnage des produits exige une surveillance de tous les instants, et le dénombrement, qui est en même temps une vérification générale du facconnage, est une opération très-longue, très-minutieuse et qui demande beaucoup de soin et d'habitude.

Ces difficultés, on le sent, sont de celles qui s'aplaniront avec le temps; mais il en est une qui subsistera toujours, parce qu'elle est inhérente au système, c'est celle qui résulte de l'établissement de la série des facteurs exprimant la relation entre les valeurs de chaque espèce de marchandise et la valeur de l'unité prise pour type. La détermination de ces facteurs est une opération extrêmement délicale, sinon pour les bois de feu, au moins pour les bois d'œuvre dont la valeur dépend non-seulement des dimensions variables des pièces, mais de la qualité tout aussi variable du bois. On peut bien faire des catégories qui comprennent exactement les pièces 
de dimensions diverses, mais il est impossible de classer d'avance les différentes qualités du bois. $\mathrm{Si}$ donc on veut éviter des appréciations arbitraires et des contestations lors du dénombrement, ce qu'il y a de mieux à faire c'est d'établir les facteurs des bois d'œuvre indépendamment de la qualité du bois. Remarquons au surplus qu'en agissant ainsi on reste exactement dans les conditions où se trouvent placés acheteurs et vendeurs, lorsqu'ils procèdent à l'estimation en matière et en argent des coupes à vendre sur pied, sauf cette différence, que nous nous plaisons à faire ressortir en faveur de ce mode d'adjudication, c'est que l'acheteur ne court jamais le risque de payer, au prix du bois d'œuvre, des arbres pourris à l'intérieur ou tellement viciés qu'ils ne sont propres qu'à donner de mauvais bois de feu. 


\title{
ESTINATION
}

\section{DE LA VALEUR DES FORÊTS}

EN FONDS ET SUPERFICIE.

\section{CHAPITRE PREMIER.}

\author{
EXPOSÉ DES PRINCIPES.
}

\section{Article I.}

Enoncé de la question.

L'estimation de la valeur en fonds et superficie d'une forêt ou d'une portion de forêt est une opération qui a pour objet de déterminer la valeur en argent qu'elle représente actuellement pour le propriétaire, ou pour le capitaliste qui serait disposé à l'acheter en vue de placer son argent en fonds de terre, aux conditions ordinaires des placements de même nature dans la localité.

Lorsqu'il s'agit d'une propriété ordinaire, d'une terre arable, d'un pré ou d'une ferme, chacun sait que sa valeur réelle, positive et commerciale, autrement dit sa valeur comme placement de fonds, abstraction faite de toute considération étrangère telle 
que la convenance dans un but d'industrie, d'agrément, ou de spéculation, chacun sait, disons-nous, que cette valeur dépend exclusivement du revenu qu'elle rapporte ou qu'elle peut rapporter, et du taux des placements en fonds de terre dans la localité. Il en est de même des propriétés forestières. Mais la culture des bois est très-différente de celle des champs; et, tandis que les fruits d'une terre arable peuvent se récolter tous les ans, la production d'un sol forestier ne peut se réaliser périodiquement qu'à des époques assez éloignées et qui peuvent varier, au gré du propriétaire, dans de très-larges limites. Or, il est évident $\dot{a}$ priori que le revenu ou la production en matière et en argent d'un bois doit singulièrement différer suivant l'âge auquel on l'exploite; que si, par exemple, un massif de chêne peut produire à 15 ans une certaine quantité de bois de feu ou de petite industrie, le même massif serait susceptible de fournir un produit matériel bien plus important, sous le rapport de la quantité et de la qualité, et par suite un revenu en argent bien plus considérable, si on reculait le terme de son exploitation de 50, 80 ou 100 ans. Par contre, on remarque aussi, à première vue, que tout retard apporté à l'exploitation d'un bois se traduit, pour le propriétaire, par la perte des intérêts de la somme qu'il aurait pu réaliser en exploitant plus tôt et par la valeur des bois qui se fussent reproduits depuis cette exploitation. D'où ce principe d'économie forestière : que pour tirer d'un bois le revenu en argent le 
plus avantageux, au point de vue de la valeur industrielle et commerciale de la propriété, il faut exploiter ce bois à l'âge où la plus-value que les produits acquièrent annuellement, cesse de dédommager le propriétaire du sacrifice qu'il fait en différant l'exploitation; et réciproquement, que pour obtenir la valeur réelle, industrielle et commerciale d'une forêt, il est nécessaire de prendre pour base de cette évaluation le revenu net que l'on peut tirer de la propriété, en exploitant les bois à l'àge où les produits satisfont le plus complétement les intérêts pécuniaires du propriétaire.

De ces considérations il résulte que, pour procéder d'une manière régulière à l'estimation de la valeur en fonds et superficie d'un bois, d'une forêt ou d'une portion de forêt, il est nécessaire de connaìtre :

$1^{\circ}$ Le taux auquel se font les placements en fonds de bois dans la localité;

$2^{\circ}$ La valeur nette du revenu à l'âge où les bois doivent être exploités, pour que les produits soient le plus profitables à l'intérêt pécuniaire du propriétaire.

Article II.

Choix du taux d'intérêt ou de placement.

Le taux de placement d'une somme placée à intérêts n'est autre chose que le rapport du revenu net au capital qui le produit. Si le capital est fixe, le 
taux varie avec le revenu et dans le même sens que le revenu. Si le revenu et le capital varient à la fois, le taux varie dans le rapport de ces deux sommes. Réciproquement, si le taux et le revenu sont fixes, le capital reste invariable; si le taux est fixe et le revenu variable, le capital varie dans le même sens que le revenu.

Le taux de placement des capitaux engagés dans l'industrie dépend ordinairement des chances commerciales attachées au genre de spéculation que l'on embrasse. Plus les risques à courir sont grands ou nombreux, plus le taux doit être élevé. C'est ainsi que dans le commerce les placements ne se font guère au-dessous de $6 \mathrm{p} .0 / 0$ et atteignent souvent le chiffre de 20 p. $0 / 0$ et plus.

Les placements en fonds de terre sont les plus solides, c'est-à-dire, les moins hasardeux ; par contre, ils donnent, relativement, les revenus les moins élevés. Le taux de ces placements peut différer avec les pays, mais il est à peu près le même dans chaque contrée, et il s'établit d'après les prix moyens qui servent de base aux transactions commerciales de même espèce. En France, le taux des placements en fonds de terre est généralement compris entre 2 et 4 p. $0 / 0$, suivant la richesse des provinces et l'intensité des populations. Ce taux peut varier, dans chaque localité, pour un temps plus ou moins long, suivant les circonstances politiques ou commerciales qui affectent le revenu des propriétés et l'emploi des capitaux; mais les oscillations du taux moyen ne 
dépassent guère $1 / 4$ ou $1 / 2 \mathrm{p} .0 / 0$; rarement l'écart atteint $1 \mathrm{p} .0 / 0$.

Il existe donc, dans chaque localité ou province d'un grand pays, un taux bien déterminé et bien connu qui sert de base aux placements des capitaux en fonds de terre, et, ce taux une fois établi, il n'appartient pas à la volonté d'un individu de le modifier. Ainsi quand, dans une localité, on dit que les terres arables rapportent $4 \mathrm{p} .0 / 0$, cela signifie que la terre vaut 25 fois le revenu net annuel que l'on en peut tirer. Réciproquement, quand la terre se vend et s'achète communément sur le pied de 25 fois le revenu net annuel qu'elle peut fournir, cela veut dire que les placements en fonds de terre se font généralement au taux de 4 p. $0 / 0$.

Eu égard au taux de placement, ce que nous disons des terres arables s'applique également aux propriétés forestières, c'est-à-dire que, dans chaque localité, le taux des placements en fonds de bois est établi d'une manière fixe et à peu près immuable. Si ce taux est de 4 p. $0 / 0$, comme celui des placements en terres arables, cela signifie que, dans la localité, les forêts peuvent être constituées, traitées et exploitées, de manière à fournir un revenu net qui, par rapport au capital producteur, représente un placement de ce capital au taux de 4 p. 0/0.

Le rapport du revenu au capital placé en terres arables ou en fonds de bois ne peut jamais être plus fort ni plus faible que le taux admis dans la localité pour les placements de même espèce. En effet, si ce 
taux est de 4 p. 0/0 et qu'un capitaliste achète pour 100000 fr. une propriété, agricole ou forestière, susceptible de produire un revenu net annuel de $5000 \mathrm{fr}$., cela prouve simplement que l'acquéreur a fait une bonne affaire; car, s'il voulait revendre sa propriété, il pourrait en trouver un prix égal à 25 fois le revenu ou $125000 \mathrm{fr}$. Si au contraire la même propriété a été payée 200000 fr., on peut affirmer, toute question de convenance à part, que l'acheteur a fait un mauvais marché. Donc si, par des soins plus intelligents ou par des améliorations quelconques apportées dans la culture, le propriétaire d'une ferme ou d'un bois parvient à augmenter le produit net de sa propriété, dans une proportion plus forte que l'intérêt des frais consacrés à son amélioration, la valeur réelle et capitale de la propriété se trouvera augmentée dans le même rapport, sans que le taux de placement cesse d'être le même. Inversement, si le revenu net d'une propriété vient à diminuer, soit par négligence ou défaut de soin du propriétaire, soit par des causes indépendantes de sa volonté, la valeur capitale de la propriété se trouvera amoindrie dans la même proportion, sans que le taux subisse aucune variation.

Donc, quelles que soient les conditions dans lesquelles la propriété se trouve placée, eu égard à sa constitution acluelle et au revenu que l'on en peut tirer, ces conditions n'ont aucune influence sur le taux de placement ou d'intérêt à employer dans les calculs d'estimation de la valeur capitale, parce que 
le taux des placements en biens-fonds dans une localité est indépendant, d'une manière générale, de la nature de la propriété à estimer.

On a quelquefois dit que le taux des placements en fonds de bois devait être plus élevé que celui des placements en fonds de terre arable, en d'autres termes, qu'une ferme avait plus de valeur qu'une forêt susceptible du même revenu, parce que les propriétés forestières ne donnent pas des produits aussi réguliers que les champs, et aussi parce que les forêts, ne pouvant pas s'affermer comme les champs, exigent toujours de la part du propriétaire une certaine somme de temps et de soin dont il peut s'affranchir à l'égard des autres propriétés, en les louant à un fermier. Cette opinion ne nous paraît pas fondée, parce que, aux raisons dont on l'appuie, on peut en opposer d'autres tout aussi concluantes pour la combattre. Et d'abord, il est clair pour tout le monde que les dangers qui menacent la production d'une forêt, tels que les incendies, les coups de vent, les ravages d'insectes, etc., trouvent leurs équivalents dans les accidents météoriques de toute sorte auxquels les récoltes des champs, des prés, des vignes sont incessamment exposées. En second lieu, si la valeur des produits forestiers est soumise à des fluctuations qui ne permettent pas de compter sur un revenu parfaitement régulier et uniforme, les récoltes des champs sont exposées à des variations analogues que le propriétaire est obligé de subir, directement s'il exploite lui-mème ou si les fer- 
mages lui sont payés en nature, indirectement par une réduction sur les prix de fermage si la propriété est exposée à des dangers périodiques prévus d'avance, ou si le fermier a à supporter un accident qui compromet le fruit de son travail. De plus, landis que les produits agricoles doivent nécessairement se récolter et se vendre à des époques à peu près fixes, quel que soit le prix de ces denrées, le propriétaire forestier peut attendre le moment favorable à l'écoulement de ses produits, en restreignant ou en suspendant ses exploitations quand le bois se vend mal, en donnant plus d'extension à ses coupes quand les produits sont recherchés. Enfin, si le propriétaire d'une forêt ne peut s'affranchir absolument de tout soin à l'égard de sa propriété, peut-on dire que le propriétaire d'une terre soit toujours exempt de souci vis-à-vis du fermier auquel il a loué ses champs, non-seulement en ce qui concerne le paiement régulier de son fermage, mais encore en ce qui touche la manière dont sa propriété est soignée, cultivée, entretenue?

On objecte encore qu'une forêt est presque toujours une propriété importante, indivisible, inaccessible aux petites fortunes et, par conséquent, difficile à vendre, parce qu'elle se trouve en dehors de la concurrence qui s'exerce toujours entre une foule d'amateurs pour l'achat des petites propriétés. Cela est vrai jusqu'à un certain point; mais remarquons d'abord, en passant, que les effets ordinaires de cette concurrence, de cette envie de posséder qui 
anime les populations des campagnes, sont de leur faire payer la terre à des prix exagérés et nullement en rapport avec le produit qu'elles en peuvent retirer. En second lieu, de ce qu'une propriété n'est accessible qu'à de grandes fortunes, il se peut bien sans doute qu'on ne trouve pas à la vendre à tout moment comme un bout de champ ou de pré, mais ce n'est pas à dire qu'elle ait moins de valeur, relativement, qu'une propriété de moindre importance ; car, si elle dépasse les forces d'un individu, elle pourra convenir à plusieurs qui s'associeront pour l'acquérir et l'exploiter. N'a-t-on pas vu en effet les capitalistes les plus intelligents rechercher avec avidité les forêts, surtout les plus importantes, que l'Etat a mises en vente dans des moments de crise financière, et les payer à des prix qui, en raison de la difficulté des circonstances politiques, n'étaient pas inférieurs à ceux qu'ils auraient pu raisonnablement donner d'une ferme susceptible du même revenu?

Ajoutons enfin qu'un propriétaire forestier peut toujours plus facilement attendre le moment favorable pour la vente de sa propriété que le propriétaire d'une vigne ou d'une ferme, parce qu'une forêt, ou même un bois quelque peu important, est presque toujours constitué de telle façon que le propriétaire peut, dans un moment de gêne ou de besoin, exploiter une partie de la superficie par anticipation, en attendant l'occasion favorable de vendre sa propriété. 
Concluons donc, qu'à moins de circonstances exceptionnelles, la propriété forestière a autant de valeur, à revenu égal, que toute autre propriété foncière, et, conséquemment, que le taux admis pour les placements en terres arables, dans une localité, est cehii dont on doit se servir dans les calculs qui ont pour objet la détermination de la valeur en fonds et superficie d'une forêt située dans la même contrée.

\section{Article 1 III.}

Recherche du revenu.

S I. - Du mode de constitution et de production des propriétés forestières.

L'estimation de la valeur capitale d'une ferme ou d'un champ est une opération qui ne peut jamais présenter de difficulté sérieuse, parce que, dans chaque localité, on peut toujours obtenir des renseignements précis sur la production moyenne des terres arables, et connaître le revenu net en argent qu'elles peuvent rapporter annuellement. Mais s'il s'agit d'un bois ou d'une forêt, la détermination du revenu net, qui doit servir de base à l'estimation de la valeur capitale de la propriété, peut donner lieu à des opérations qui exigent de la part de l'estimateur une connaissance théorique et pratique des questions les plus délicates de l'économie forestière. Pour apprécier cette difficulté et se faire une idée 
nette des moyens à employer pour la résoudre, il est nécessaire de se rendre compte d'abord de la différence entre le mode de constitution et de production des propriétés forestières et celui des champs, des prés, etc.

On sait que la production d'un sol boisé ne peut se récolter tous les ans comme celle d'un pré, par exemple, parce que la matière ligneuse n'acquiert de valeur réelle et utilisable, comme produit forestier, qu'après un nombre d'années plus ou moins grand, suivant les essences, les climats, les terrains et les localités. Ces produits se forment par accroissements annuels et successifs de la matière ligneuse, de la même manière que s'accroît un capital placé à intérêts composés, à cette seule différence près que les capitaux monétaires fonctionnent suivant une loi déterminée et mathématique, tandis que l'accroissement annuel d'un bois et de la valeur en argent qu'il représente à chaque âge ne suit jamais une marche régulière et qui puisse être définie en termes rigoureux. L'accroissement ligneux, autrement dit la pousse ou la feuille de chaque année, est une fonction double du fonds de terre et des accroissements antérieurs ou, en traduisant en argent, la plus value que le bois acquiert chaque année est le produit du capital qui représente la valeur du fonds et celle des accroissements ligneux antérieurs, tout comme l'accroissement annuel d'une somme placée à intérêts composés est le produit du capital primitif et des intérêts accumulés depuis le jour où le placement a été fait. 
Un propriétaire qui plante son champ en bois ne pourra donc en tirer un revenu qu'après un certain nombre d'années, 10 ans par exemple, si, avant cet àge, les bois n'ont pas de valeur commerciale comme produits forestiers. Avant cette époque, la superficie n'aura qu'une valeur d'avenir, laquelle dépendra de celle que les produits pourront fournir lorsqu'ils seront réalisables. D'où l'on voit, qu'avant de tirer aucun profit de sa plantation, le propriétaire est obligé de constituer un capital qui se compose au début de la valeur du fonds de terre, et qui s'accroît successivement, année par année, de la plus-value qu'acquiert le matérie! ligneux. Quant au revenu net à réaliser au moment de l'exploitation, c'est-à-dire à 10 ans, il sera égal à la valeur sur pied des bois à exploiter, diminuée de la dépense que l'on pourra être obligé de faire pour le repeuplement partiel ou total du terrain après l'exploitation et des frais, accumulés avec intérêt, que l'on aura été forcé de débourser annuellement pour l'entretien, la garde et l'impôt depuis la naissance du massif.

Maintenant, si au lieu d'un bois peuplé tout entier d'arbres du même àge et périodiquement exploitable tous les 10 ans, nous considérons une forêt formée de peuplements d'âges gradués et régulièrement aménagée à la même révolution, chaque année on pourra récolter un produit uniforme égal à l'accroissement moyen de toute la superficie. Ainsi constituée, la propriété devient parfaitement assimilable à une ferme qui rapporte le même revenu tous les 
ans; mais, pour assurer la perpétuité du revenu d'une forêt ainsi aménagée, on voit que, à côté de la coupe âgée de 10 ans que l'on exploite chaque année, il faut entretenir et ajouter au capital du fonds de terre un capital superficiel représenté par la valeur d'avenir des coupes âgées de 1 à 9 ans. Quant à la valeur nette du revenu annuel, on peut la considérer comme étant égale au produit moyen de la vente des coupes sur pied, diminution faite des frais que l'on est obligé de débourser annuellement pour la culture, l'entretien, la garde et l'impôt.

Que si enfin le propriétaire réserve, dans chaque coupe, un certain nombre d'arbres destinés à parcourir plusieurs révolutions et à acquérir de fortes dimensions, on voit encore, par analogie avec les cas précédents, que la valeur de ces réserves s'ajoutera au capital producteur, tandis que la valeur de celles qui seront abattues dans chaque coupe feront partie du revenu de la propriété.

Les considérations qui précèdent font voir de quelle manière se constitue le capital producteur des propriétés forestières, et comment se forme le revenu ou le produit à récolter après un temps déterminé. C'est le rapport de ces deux quantités, autrement dit la proportion du revenu net au capital engagé, qui donne la mesure du bénéfice que le propriétaire retire de sa culture. Or, on comprend à priori que si, au lieu d'exploiter son bois à 10 ans, le propriétaire se décide à reculer le terme d'exploitation à 15 ans, à 20 ans, etc., le revenu et le ca- 
pital producteur s'accroîtront simultanément dans des proportions qui pourront être différentes, et si, comme précédemment, on compare le revenu à 15 ans, à 20 ans, etc. avec le capital qui a servi à le produire, on sera conduit à choisir pour terme d'exploitation l'âge auquel le revenu sera le plus grand possible par rapport au capital producteur. Cet âge correspond à ce qu'on appelle, en économie forestière, le terme de Texploitabilité commerciale ou relative à la rente la plus élevée.

D'où l'on conclut, réciproquement, que pour procéder d'une manière exacte à l'estimation de la valeur en fonds et superficie d'une propriété forestière, il faut prendre pour base de cette estimation le revenu net qu'elle pourra fournir, en exploitant les bois à l'âge où iis ont atteint le terme de leur exploitabilité commerciale.

Ces principes généraux posés, nous allons voir comment on procède à la fixation du terme de l'exploitabilité commerciale d'un bois ou d'une forêt et, par suite, comment on détermine le revenu qui doit servir de base à l'estimation de la valeur capitale de la propriété.

SII. - Fixation du terme de l'exploilabilité commerciale et du revenu correspondant d'un bois peuplé d'arbres de même âge. (Taillis simples, futaies régulières.)

Supposons d'abord que l'on ait à opérer sur une portion de forêt ou sur un bois peuplé tout entier d'arbres de même âge, destiné à être exploité pério- 
diquement à des intervalles égaux et parvenu dès à présent au terme de son exploitabilité minima, c'està-dire à l'âge où les produits, en raison de leurs dimensions et de leurs qualités, commencent à avoir une valeur commerciale assurée. Supposons en outre, pour fixer les idées, que ce bois ait actuellement 10 ans et que l'on se demande s'il peut y avoir avantage, au point de vue pécuniaire, à l'exploiter tous les 15 ans plutôt que tous les 10 ans ?

La théorie (1) répond d'une manière générale à cette question que le terme de l'exploilabilité commerciale sera atteint, lorsque le gain qui résulte du retard apporté à l'exploitation cessera de dédommager le propriétaire du sacrifice ou de la perte qu'il fait en différant sa coupe. Or, au cas particulier, le gain que réalisera le propriétaire, en retardant de 5 ans l'exploitation de son bois, sera représenté par la différence entre les valeurs nettes de la superficie à 15 ans et à 10 ans, tandis que la perte qui résulte pour lui de la même opération comprendra : $1^{\circ}$ les intérêts composés pendant 5 ans de la somme qu'il aurait pu réaliser en exploitant son bois à 10 ans; $2^{\circ}$ la valeur d'avenir des 5 premières feuilles ou pousses qui se seraient reproduites, si l'exploitation avait eu lieu 5 ans plus tôt. Si donc on effectue ces calculs et que l'on compare entre elles les valeurs du gain et de la perte, on verra s'il y a avantage ou

(1) Voyez le Cours de culture des bois de MM. Lorentz et Parade, livre second, chapitre $1^{\mathrm{er}}$, article IV. 
non à reculer le terme de l'exploitation jusqu'à 15 ans. Si la différence entre le gain et la perte est négative, on en conclura que la révolution de 10 ans est préférable à celle de 15 ans; si elle est nulle, cela prouvera qu'il n'y a ni avantage ni perte à exploiter soit à 10 ans, soit à 15 ans; si enfin elle est positive, on sera conduit à se demander s'il n'y aurait pas avantage à reculer encore le terme d'exploitation, auquel cas on opérerait de la même manière sur les résultats de l'exploitation à 1 à ans et à 20 ans, et ainsi de suite jusqu'à ce que l'on trouvât qu'il n'y a plus aucun avantage pour le propriétaire à différer sa coupe.

Si simple que celte théorie paraisse, elle présente des difficultés très-sérieuses dans son application, notamment en ce qui concerne la valeur à assigner au second élément de la perte ou, au cas particulier, aux cinq premières feuilles ou pousses qui se fussent reproduites si l'exploitation avait eu lieu à 10 ans. Mais on peut éviter les difficultés ou les objections qui peuvent naître du calcul de la perte, et arriver directement à la solution du problème qui nous occupe par une opération arithmétique très-facile.

En effet, rappelons-nous qu'il s'agit de fixer le terme d'exploitabilité d'un massif d'arbres de même âge, de façon à en tirer le produit le plus profitable à l'intérêt pécuniaire du propriétaire; autrement dit, il s'agit de régler les conditions de la production, de manière à donner à la propriété la plus grande valeur vénale qu'elle puisse atteindre, 
comme propriété de rapport ou comme placement de fonds. Si le bois sur lequel on opère devait être exploité indéfiniment à l'âge de 10 ans, sa valeur en fonds et superficie, au début de la révolution, ou immédiatement après une exploitation, serait égale au capital qui, placé à perpétuité au taux des placements en biens-fonds dans la localité, serait susceptible de produire, en intérêts seulement, tous les 10 ans, une somme égale à la valeur nette de la superficie à 10 ans. De même si, au lieu de 10 ans, le terme d'exploitation devait être fixé à 15 ans, la valeur capitale de la propriété, au début de la révolution, serait égale à la somme qui, placée au même taux, serait susceptible de fournir, en intérêts seulement, tous les 15 ans, un revenu égal à la valeur nette de la superficie à 15 ans.

On voit suffisamment par là comment on devrait opérer pour déterminer la valeur capitale la plus grande que l'on pourrait offrir d'une telle propriété comme placement de fonds. Or, à cette valeur maxima correspond évidemment la production la plus lucrative et, par conséquent, le terme d'exploitation le plus avantageux. Donc, en résumé : Pour fixer le terme de l'exploitabilité commerciale d'un bois à exploiter périodiquement, il suffit de connaître les valeurs nettes de la superficie à tous les âges postérieurs à l'époque où les produits deviennent commerçables, de capitaliser chacun de ces revenus comme une rente périodique à toucher pour la première fois à l'âge correspondant, et de prendre pour 
terme d'exploitabilité l'âge auquel la valeur capitale est maxima.

Cette manière de fixer le terme de l'exploitabilité commerciale d'un bois peuplé tout entier d'arbres de même âge peut également s'appliquer aux forêts aménagées en coupes annuelles, telles que les taillis simples et les futaies régulières, dont tous les produits de chaque exploitation sont fournis par des bois d'un âge uniforme. Il est clair, en effet, que si on expérimente sur un peuplement placé dans les conditions moyennes de production d'une forêt ou d'une série d'exploitation ainsi constituée, le propriétaire aura intérêt à exploiter au même âge tous les massifs de la série qui végèteront dans les mêmes conditions. D'ailleurs on pourrait toujours, à l'aide d'un calcul d'escompte, obtenir la valeur actuelle des revenus successifs à toucher annuellement, pendant chacune des révolutions auxquelles la forêt peut être soumise, et déterminer le capital équivalent à la somme de ces revenus escomptés, cette somme étant considérée comme un produit unique à toucher périodiquement à l'âge marqué par la révolution correspondante; il ne resterait plus alors qu'à comparer entre elles les valeurs capitales correspondantes à chaque révolution, comme on le fait pour les bois à exploitations périodiques.

Tel est le procédé, éminemment simple, à l'aide duquel on peut fixer le terme de l'exploitabilité commerciale d'un bois dont tous les produits sont fournis par des arbres de même âge. C'est le revenu 
net que l'on obtiendrait en exploitant les bois à cet âge qui doit servir de base à l'estimation de la valeur capitale de la propriété. Pour déterminer ce revenu, on voit qu'il est nécessaire de savoir évaluer le rendement probable que l'on pourrait tirer de la superficie, en exploitant les bois à des époques plus ou moins éloignées de l'âge actuel. Or, il n'y a ni théorie, ni règle précise à tracer pour procéder à l'évaluation de la production probable d'un bois à un âge quelconque; il n'y a que l'expérience, c'està-dire, la connaissance acquise des lois de la végétation, qui puisse servir de guide en cette matière; car, soit que l'on ait à évaluer la production future de jeunes bois, soit que l'on ait à rechercher ce que valait la superficie à des époques antérieures à l'âge du peuplement sur lequel on opère, tous les procédés que la théorie indique, comme servant à la détermination de l'accroissement futur ou passé des bois, ne sont que des moyens plus ou moins empiriques et qui exigent implicitement, de la part de ceux qui les emploient, beaucoup de tact et de discernement, un coup d'œil exercé, en un mot toutes les qualités d'un forestier expérimenté.

On comprend d'après cela que le terme de l'exploitabilité commerciale d'un bois ne peut jamais s'obtenir avec une exactitude précise et mathématique, et que cet âge peut varier pour chaque forêt avec les essences, les conditions de la végétation et l'utilité ou la valeur que l'on assigne aux produits dans chaque localité, suivant les dimensions et la 
qualité des bois. Mais pour les forestiers vraiment habiles, et nous entendons par là ceux qui à la connaissance des lois de la végétation réunissent celle des circonstances économiques qui régissent la production des forêts, la fixation du terme de l'exploitabilité commerciale et du revenu probable à cet âge ne présentera jamais de difficulté sérieuse, parce qu'ils sauront écarter, parmi les données du problème, celles qui n'offrent qu'un caractère aléatoire, et ne voudront baser leurs recherches et leurs évaluations que sur des faits positifs et certains (1). Ajoutons, au surplus, que dans beaucoup de localités, le terme de cette exploitabilité se trouve assez exactement fixé par l'àge auquel les particuliers exploitent leurs forêts.

(1) Nous n'entendons pas dire que la valeur capitale d'une forêt doive, dans tous les cas, se déduire strictemeni du revenu à l'âge d'exploitabilité des peuplements existants, quels que soient l'état de végétation et la consistance de ces peuplements. Au contraire, toutes les fois que l'on pourra apprécier la plus value que le revenu sera susceptible d'atteindre dans l'avenir, il sera nécessaire d'en tenir compte dans l'estimation de la valeur capitale de la propriété, de même qu'il faudra tenir compte de la dépense à faire en travaux d'amélioration pour obtenir ce résultat. C'est ainsi, par exemple, qu'il faudrait procéder à l'égard de vides importants, de peuplements compromis par des exploitations vicieuses, de massifs réduits à végéler misérablement faute de quelqques travaux d'amélioration, tels que fossés d'assainissement, etc., etc., en un mot, de tous les peuplements dont la production peut être améliorée par des moyens certains, et dont la plus value nette, au moment des exploitations à venir, peut s'apprécier avec certitude. De même, si les ressources d'une forêt ont été épuisées ou amoindries par des exploitations exagérées, il est évident que le revenu qu'elle a fourni en dernier lieu ne peut être pris pour base de l'estimation de sa valeur caitale. 
SIII, - Fixation du terme de l'exploilabiliié commerciale et du revenu à cel âge d'un peuplement composé de bois d'âges différents (Taillis composés, futaies jardinées, etc.).

Voyons maintenant comment il faudrait procéder à la détermination du revenu qui doit être pris pour base de l'estimation si, au lieu d'un bois ou d'une forêt dont chaque coupe ne comprend que des bois de même âge, on avait à opérer sur un peuplement dont les produits, annuels ou périodiques, devraient être fournis par des arbres d'âges différents, un taillis sous futaie, par exemple, ou une futaie jardinée.

Dans un taillis sous futaie, le rendement de chaque coupe se compose de deux éléments : du produil de tout le sous-bois, et du produit de ceux des arbres de réserve qui sont abandonnés à l'exploitation. Pour ce qui est du sous-bois, on procède, comme pour les taillis simples, à la détermination du revenu net qu'il peut fournir à l'àge où il a atteint son exploitabilité commerciale. Seulernent on doit remarquer que la révolution à lui appliquer doit être déterminée, eu égard aux essences et aux conditions de fertilité, de façon à assurer la bonne venue des arbres de réserve en même temps que la reproduction des souches du taillis. Plus la révolution des taillis est longue, plus beaux sont les résultats que l'on obtient de la réserve, et comme le contingent de la futaie peut entrer pour une forte part, et le plus 
souvent même pour la plus forte part, dans le produit total de chaque coupe, il peut être de l'intérêt du propriétaire de favoriser plus spécialement le développement des arbres de réserve en reculant l'âge d'exploitation du sous-bois au-delà du terme de son exploitabilité commerciale. Cet âge que l'expérience a fixé, pour nos principales essences et suivant les sols, entre les limites de 20 à 25 ans au minimum et 40 ans au maximum, pourra se déterminer, en faisant abstraction de la réserve, de la manière qui a été tracée pour les taillis simples, mais en se maintenant entre les limites que nous venons d'indiquer.

Reste donc à apprécier la part pour laquelle la futaie doit contribuer au rendement total de chaque coupe. Or, on sait que dans un taillis sous futaie la réserve se compose d'un certain nombre d'arbres ou baliveaux qu'on laisse sur pied, à chaque exploitation du taillis, et que l'on classe suivant leur âge en catégories différentes. Les baliveaux de lâge sont des sujets que l'on choisit parmi les plus belles perches du taillis et qui ont le même âge que celui-ci au moment de son exploitation; les baliveaux modernes sont des arbres de deux révolutions; les baliveaux anciens sont de plusieurs sortes et se divisent en anciens de trois âges, anciens de quatre àges, etc. Dans les forêts bien traitées, le nombre des baliveaux de chaque catégorie à réserver, lors de l'exploitation, est réglé par un plan de balivage conçu de façon à pourvoir aux exigences de la cul- 
ture (1) et à satisfaire les intérêts du propriétaire. Le surplus de ces arbres est destiné à l'exploitation et, par conséquent, à fournir le contingent de la réserve dans le produit de la coupe. Dans ce cas, et dans toutes les circonstances où l'on opère sur un taillis composé que l'on considère comme parfaitement constitué, la part pour laquelle la futaie contribue au rendement de chaque coupe est facile à estimer; car si, après l'exploitation du tailìis, la réserve se compose, par exemple, de 50 baliveaux de l'âge, 40 modernes, 30 anciens de trois âges et 20 anciens de quatre âges, il est clair que la part de la futaie dans le rendement de l'exploitation suivante sera représentée par le produit de 20 anciens de 5 âges, 10 anciens de 4 âges, 10 anciens de 3 âges et 10 modernes.

Mais si les conditions qui doivent assurer la meilleure production du peuplement à estimer ne sont pas convenablement remplies, en ce qui concerne la constitution de la réserve, il peut être nécessaire de tenir compte des améliorations qui devront être introduites sous ce rapport et de la plus value qui en résultera dans la production à venir. Pour mieux faire comprendre la marche générale à suivre dans l'estimation de peuplements de cette nature, nous allons d'abord nous supposer placé dans la position d'un propriétaire qui veut élever des réserves dans un taillis simple, et examiner jusqu'à quel point

(1) Voyez le Cours de culture des bois, livre IV, chapitre II. 
cette opération peut être utile ou préjudiciable à ses intérêts.

Considérons un massif de taillis simple parvenu au terme de son exploitabilité commerciale que nous supposerons être 25 ans. Si, en abattant le taillis, le propriétaire veut réserver un certain nombre de brins de chêne, par exemple, pour ne les exploiter que lorsqu'ils seront propres à donner du bois d'œuvre, cette opération aura pour effet de diminuer le revenu de la coupe d'une quantité égale à la valeur des brins réservés (1).

A l'exploitation suivante du taillis, les réserves seront âgées de 50 ans, et auront une valeur immédiatement réalisable qu'il sera facile de déterminer. Pour se rendre compte de l'intérêt que le propriétaire peut trouver à élever ces réserves sur son taillis, on remarqnera d'une part que si ces arbres avaient été exploités $2 \check{5}$ ans plus tôt, le prix que l'on en aurait tiré aurait pu être placé dans les mêmes conditions de solidité que les capitaux en fonds de bois

(1) Ces brins, croissant en quelque sorte isolément au milieu des rejets du taillis, recevront de toutes parts les influences de la lumière; d'ailleurs, le sol qui les porte sera protégé par le courert du sous-bois et enfin, à partir de 25 ans, ils entreront dans un âge plus favorable à leur développement; d'où il résulte en somme que les conditions dans lesquelles ces réserves vont végéter, ne sont pas les mèmes que celles où vont se retrouver les sujets qui repousseront de souche et qui formeront la masse du sous-bois. Ces arbres, considérés individuellement ou en masse, prendront annuellement plus d'accroissement qu'ils n'en ont pris dans les 25 premières années de leur vie, et qu'ils n'en auraient pris si on avait laissé croître le massif entier du taillis jusqu'à 50 ans. 
dans la localité, d'autre part que les arbres réservés pendant une seconde révolution ont pu causer un certain préjudice au sous-bois et amoindrir le revenu du taillis. La somme des intérêts perdus et du préjudice causé au sous-bois, mise en regard de la plus value que les réserves auront acquise pendant la seconde révolution, fera connaître le bénéfice ou la perte qui résultera pour le propriétaire de la constitution d'une réserve sur son taillis. S'il y a bénéfice, le propriétaire continuera à faire une réserve en baliveaux de l'âge et pourra être tenté de laisser les modernes parcourir une troisième révolution du taillis ; mais en même temps il pourra être amené à réduire le nombre des arbres réservés comme modernes, afin de ne pas compromettre la venue du taillis par un couvert trop considérable. En comparant, comme précédemment, le sacrifice qu'il s'impose en réservant un certain nombre de modernes avec la plus value que ces arbres auront acquise à 75 ans, le propriétaire pourra apprécier le profit ou la perte qui résultera pour lui de cette seconde opération. Cette expérience répétée sur les baliveaux de toutes catégories qui peuvent composer la réserve dans un taillis sous futaie le conduira à fixer le nombre des baliveaux de chaque catégorie à réserver et à abattre lors de chaque coupe, et à prendre pour terme d'exploitabilité des réserves les plus anciennes l'àge où la plus value nette qu'elles prendraient, si on les laissait 25 ans de plus sur pied, cessera de le dédommager du sacrifice qu'il s'impose en ne les exploitant pas. 
Donc, réciproquement, quand on veut estimer la valeur en fonds et superficie d'une forêt ou d'une portion de forêt peuplée d'un taillis composé, il faut d'abord déterminer l'âge correspondant à l'exploitabilité commerciale du taillis considéré séparément, et le revenu qu'on en pourra tirer à cet âge. Passant ensuite à l'examen de la réserve, on appréciera, comme il vient d'être dit, l'intérêt que peut présenter l'éducation de réserves de 2 , de 3 , de 4 , etc. révolutions, et l'on fixera l'âge que les réserves ne peuvent dépasser sans perte pour le propriétaire. Cet âge représentera le terme de l'exploitabilité commerciale des réserves, ce qui veut dire que toutes celles qui auront atteint ou dépassé cet âge devront être considérées comme immédiatement exploitables, et que tous les baliveaux moins âgés, quoiqu'ayant une valeur immédiatement réalisable, puisqu'ils peuvent fournir des produits commerçables, ne sont pas encore exploitables, dans la saine acception du mot, parce qu'ils ont, à ce moment même, une valeur d'avenir plus grande que celle que l'on pourrait réaliser en les exploitant.

Quant à la part pour laquelle la réserve contribuera à la formation du revenu, on la déduira du plan de balivage adopté (1), en estimant pour leur

(1) La constitution du plan de balivage est l'opération la plus délicate du traitement des taillis composés, et varie avec les circonstances physiques et économiques dans lesquelles la forêt se trouve placée. Cette question ne saurait être traitée ici sans trop nous écarter de notre sujet, et comme nous avons posé en fait qu'on ne pouvait procéder, d'une ma- 
valeur réalisable, au moment de la coupe du taillis, les modernes, anciens, etc., qui devront être abandonnés à l'exploitation.

On voit, d'après ce qui précède, que l'estimation de la valeur en fonds et superficie des taillis composés présente des difficultés que nous n'avions pas rencontrées dans les forêts où le reverıu, annuel ou périodique, est fourni par une coupe dont tous les bois sont de même âge. Ces difficultés, nous le disons en passant, sont telles qu'elles ne peuvent être abordées et sérieusement résolues que par des hommes de métier qui, à un coup d'œil exercé et à des connaissances pratiques suffisantes, réunissent l'appui indispensable d'une théorie solide. En effet, nous avons dit plus haut que, dans la fixation du terme d'exploilabilité de la réserve, il fallait tenir compte de la valeur réalisable des baliveaux de chaque catégorie, déduction faite du préjudice causé par leur présence au taillis environnant. Or comment estimer ce préjudice?

Si on considère un moderne d'abord, on peut se dire rigoureusement que cet arbre aurait pu être exploité à 2 s ans, et remplacé par un arbre qui aurait actuellement même valeur que lui. De sorte

nière sûre, à l'estimation de la valeur en fonds et superficie de ces sortes de forêts sans être un forestier consommé, nous devons admettre que tout estimateur, qui accepte la responsabilité de telles opérations, a une connaissance exacte des préceptes posés par les maîtres auxquels nous avons déjà plusieurs fois renvoyé nos lecteurs. (Voir le Cours de culture des lois de MM. Lorentz et Parade, livre IV, chap. II.) 
que, pour qu'il y ait eu intérêt à le laisser croître jusqu'à 50 ans, il faut qu'à cet âge le moderne ait une valeur plus grande que celle du baliveau de l'âge et de ses intérêts accumulés pendant 25 ans, plus le tort causé au taillis pendant le cours de la dernière révolution. Pécuniairement, ce tort serait égal à la valeur d'un brin de 25 ans, plus le dommage causé au sous-bois par le couvert du moderne. De même le préjudice causé au taillis par un ancien serait égal à la valeur en argent d'un brin de 25 ans qui aurait pu remplacer cet arbre, s'il avait été exploité comme moderne et du tort apporté à la végétation du sous-bois par son couvert. Telle serait l'appréciation rigoureuse et mathématique que l'on pourrait faire du préjudice causé par le maintien d'une réserve pendant la dernière révolution du taillis; mais, dans la pratique, on doit négliger de tenir comple, dans l'évaluation de ce préjudice, de la valeur du brin de l'àge qui aurait pu remplacer la réserve, si on l'avait exploitée 25 ans plus tôt, de même qu'on néglige de tenir compte de ce qu'on pourrait ajouter à la production possible du sol en supposant que tout le terrain est peuplé de la manière la plus uniforme et la plus complète (1). Reste donc à considérer, comme préjudice, la valeur du dommage causé au sous-bois par le couvert des arbres de réserve.

(1) On peut d'autant plus négliger la valeur du brin de l'âge que souvent elle n'atteint pas 50 centimes et ne dépasse guère 50 ou 60 centimes. 
Ce dommage varie avec les essences qui forment la réserve et dont se compose le sous-bois; il varie aussi avec la qualité du terrain, le nombre des arbres réservés et la catégorie de réserve, moderne, ancien, etc. que l'on considère. Ainsi les réserves à feuillage léger, à quelque catégorie qu'elles appartiennent, ne causent qu'un dommage insignifiant quand les essences qui forment le sous-bois redoutent peu le couvert, que le terrain est fertile et que d'ailleurs le nombre des arbres réservés n'est pas trop grand. Tel serait, par exemple, un balivage modéré en chêne sur un sous-bois de hêtre et charme, dans un terrain fertile. Si, au contraire, le couvert de la réserve est épais, il sera d'autant plus préjudiciable au sous-bois que les arbres réservés seront plus vieux, plus nombreux, moins élancés, et que le taillis a plus besoin de lumière pour végéter. C'est ainsi que le vide se fait le plus souvent sous les réserves de hêtre quand elles arrivent à la catégorie d'anciens, tandis que le sous-bois reste tout aussi fourni sous le couvert de vieux chênes, et n'éprouve souvent qu'un ralentissement insignifiant dans sa végétation lorsque, nous le répétons, le balivage est réglé d'une manière convenable. En résumé, ce dommage s'apprécie en estimant la différence entre le revenu présumé que fournirait le taillis, s'il était débarrassé du couvert de la réserve, et le revenu réel qu'il procure comme sous-bois dans le traitement en taillis composé (1).

(1) Longtemps on a pu dire que le régime du taillis simple convenait 


\section{Si l'estimation des taillis composés exige, comme} on vient de le voir, des connaissances spéciales en théorie et en pratique, il est bien plus difficile encore de procéder à l'estimation de la valeur en fonds et superficie des futaies jardinées. Comme dans toutes les opérations du même genre, le chiffre du revenu correspondant à l'exploitabilité conmerciale devra servir de base à l'estimation de la valeur capitale de la propriété. Ici le terme de l'exploitabilité

surtout à l'intérêt particulier ou, d'une manière générale, que les produits des taillis simples répondaient mieux au but de l'exploitabilité commerciale que les produits des taillis composés, parce que la constitution d'une réserve exigeait l'emploi d'un capital engagé trop fort par rapport au revenu qu'on pouvait en tirer. Cette assertion peut encore être vraie pour certains taillis croissant sur des sols maigres et peu profonds, mais il est incontestable aujourd'lıui que dans tous les taillis situés en bon fonds, et dans les localités où le bois d'œuvre a quelque valeur, il y a intérèt, au point de vue du placement des capitaux en fonds de terre, à élever de la futaie de chène éparse sur le sous-bois.

Les circonstances qui favorisent surtout le maintien ou l'introduction du mode de traitement en taillis composé, dans les forêts possédées par les particuliers, sont les suivantes :

Les bois de chêne de fortes dimensions sont devenus rares en France, depuis un demi-siècle, pour bien des causes qu'il est inutile d'énumérer ici, et ont acquis une valeur relativement plus grande que celle des bois de feu. Cette valeur tend à s'accroître encore chaque jour avec les besoins de la consommation et surtout avec l'amélioration incessante des voies de transport, tandis que les bois de feu ne peuvent pas profiter au mème degré de l'extension des débouchés et de l'économie apportéc dans les transports par la création des nouvelles voies de communication, parce que, dans les localités où leur prix tendrait à s'élever au-dessus de certaines limites, ils se trouvent en concurrence avec la houille et le coke dont l'usage se répand de plus en plus et menace sérieusement d'envahir les foyers industriels et domestiques au détriment du combustible forestier. 
ne peut plus se déduire de la valeur des arbres à un âge déterminé, mais seulement des dimensions qu'ils doivent offrir pour être propres à l'emploi de leur destination, car les arbres croissant à l'état jardiné atteignent les dimensions qui les rendent exploita. bles à des âges très-différents, suivant les conditions dans lesquelles ils ont crû. Or, quelles seront ces dimensions? Cela dépendra de la nature des marchandises qui ont le cours le plus régulier dans la localité; si ce sont, les sciages, par exemple, comme on peut scier en planches du commerce les arbres les plus forts aussi bien que ceux qui ont atteint strictement les dimensions requises pour ce genre de débit, il conviendra d'examiner si le propriétaire a intérêt à laisser les arbres sur pied jusqu'à ce qu'ils aient atteint leurs plus fortes dimensions commerciales, ou s'il doit les abattre aussitôt qu'ils ont les dimensions voulues pour être propres au sciage, ou bien s'il doit choisir un terme intermédiaire entre ces limites extrêmes. Ces dimensions étant déterminées, on considérera comme exploitables immédiatement les arbres qui les ont atteintes ou dépassées, puis on calculera la possibilité et le revenu probable de la forêt, en tenant compte du changement qui sera apporté dans la constitution des peuplements par l'extraction des arbres exploitables.

Cette opération est extrêmement délicate et difficile, parce que les produits de chaque coupe ne se composeront pas seulement d'arbres exploitables, mais encore de brins plus faibles dont les dimen- 
sions et la valeur vénale sout très-variables. On ne peut donc procéder que d'une manière empirique à la détermination de la possibilité matérielle et du revenu probable des futaies jardinées; et, pour peu que l'on connaisse ces sortes de forêts, on reconnaîtra qu'il faut avoir des notions bien exactes sur le déhit des bois et sur les lois de la végétation dans la localité où l'on opère, pour ne pas commettre d'erreur grave dans l'estimation de leur valeur en fonds et superficie. En général, le mojen le plus sûr de procéder à la détermination de la possibilité matérielle des futaies jardinées consiste à prendre pour base, ou pour point de comparaison, le chiffre moyen des produits effectifs fournis par les exploitations jardinatoires des dernières années. 


\section{CHAPITRE DEUXIËME.}

APPLICATION GÉNÉrale DES PRINGipes EN MATière D'ESTIMATION DE FORÊTS EN FONDS ET SUPERFICIE.

\section{Connaissant :}

$1^{\circ}$ Le taux des placements en biens fonds dans la localité ;

$2^{\circ}$ Le terme de l'exploitabilité commerciale de la forêt ou de la portion de forêt sur laquelle on opère, et le revenu net que l'on peut tirer de chaque coupe à cet âge ;

La détermination de la valeur capitale de la propriété ne sera plus qu'une affaire de calcul à laquelle on procèdera de la manière que nous allons indiquer.

\section{I.}

Si le produit est uniforme, annuel et continu, la valeur de la propriété s'obtiendra par une simple capitalisation du revenu net, c'est-à-dire, du produit en argent diminué des frais annuels de garde, d'impôt, etc.

Si le revenu est uniforme et périodique, comme serait le produit d'un bois périodiquement exploitable tous les 25 ans, il y a à distinguer le cas où le premier revenu ne devrait être perçu qu'au bout de 
la période de 25 ans, et celui où le premier revenu devrait être touché plus tôt. Si le premier revenu ne doit être touché que dans 25 ans révolus, ce qui suppose que le sol est nu ou couvert de jeunes semis s'il s'agit d'une futaie, ou que le bois vient d'être exploité s'il s'agit d'un taillis, la question se réduira à calculer la valeur du fonds. Or, la valeur d'un fonds de bois est égale au capital qui, placé à perpétuité, serait susceptible de fournir, aux époques fixées par le terme d'exploitabilité, une sommè d'intérêts équivalente au revenu net de ce bois. D'où il suit que, connaissant ce revenu net, il suffit de le capitaliser comme une rente périodique à toucher tous les 25 ans, pour avoir la valeur réelle de la propriété (1). Mais si le premier revenu peut être perçu plus tôt, au bout de 10 ans par exemple, ce qui revient à dire que le bois est actuellement âgé de 15 ans, on estimera comıme précédemment la valeur du fonds, on lui ajoutera la valeur nette de la superficie à 25 ans, et la somme représentera ce que vaudra la propriété dans 10 ans. Pour avoir sa valeur actuelle, il suffira d'escompter cetle somme pour 10 ans.

(1) Ici le revenu net s'obtient en retranchant du produit de la coupe exploitable les frais accumulés avec intérêts que le propriétaire est obligé de faire dans l'intervalle de deux exploitations. Mais on peut obtenir autrement la valeur nette de la propriété, en retranchant du capital correspondant au revenu brut, ou à la valeur des bois sur pied à 25 ans, le capital susceptible de fournir annuellement, en intérêts seulement, une somme égale à celle des frais annuels de garde, d'impôt, etc. 


\section{II.}

Si le revenu est variable, on doit considérer la superficie d'une part, le sol d'autre part, et calculer séparément leur valeur d'après les données de la question.

Estimation de La superficie. - Pour ce qui concerne l'estimation de la superficie, nous remarquerons que, dans une forêt quelconque, les bois sur pied peuvent toujours se partager en deux groupes distincts, savoir :

$1^{\circ}$ Les bois qui ont atteint ou dépassé le terme de leur exploitabilité commerciale ;

$2^{\circ}$ Ceux qui ne sont point encore parvenus à cette exploitabilité et n'ont, par conséquent, qu'une valeur d'avenir (1).

La première chose à faire sera donc de fixer le terme de cette exploitabilité qui variera selon les localités, selon l'essence, selon la croissance des bois et la destination des produits. Cet âge étant fixé, l'estimation du matériel sur pied devient facile.

En effet, pour les bois qui ont atteint ou dépassé le terme de cette exploitabilité, nous r'avons plus qu'à en estimer le volume, et à appliquer aux différentes parties des arbres (troncs, cimeaux et

(1) Annales forestières. T. V, p. 269. Principes pour l'estimation en fonds et superficie, par M. Parade, Directeur de l'école impériale forestière. 
branches) les prix marchands, déduction faite des frais d'abatage, de façonnage, de transport, etc. En même temps nous avons à apprécier si la valeur de ces bois peut être réalisée en une seule année; ou bien si, en raison de leur quantité, l'acquéreur aura besoin d'un certain laps de temps pour consommer son opération, auquel cas il y aura lieu de tenir compte, d'une part de la perte d'intérêts qu'il éprouvera avant de rentrer intégralement dans son capital, et d'autre part de l'accroissement que les bois'prendront jusqu'au moment de leur abatage.

Pour les bois n'ayant point encore atteint l'âge d'exploitabilité, la question se réduit à rechercher quel sera, à cet âge, leur produit matériel et la valeur vénale de ce produit, puis à calculer, par une simple opération d'escompte, ce que vaut aujourd'hui cette somme.

Estimation du fonds. - La valeur d'un fonds de bois, nous l'avons déjà dit, est égale au capital qui, placé à intérêts composés, au taux ordinaire des placements de même nature dans la localité, serait susceptible de fournir, aux époques fixées par le terme d'exploitabilité, une somme équivalente au revenu net de ce bois. Mais, dans l'estimation que nous venons de faire de la superficie, on remarquera que nous avons déjà supputé la valeur du fonds pour tout le temps qui s'écoulera depuis le moment actuel jusqu'à l'époque assignée pour l'exploitation des peuplements existants, car on a compris dans cette 
estimation non-seulement le matériel actuellement sur pied, mais encore le volume dont les bois s'accroîtront jusqu'au moment de leur exploitation. II résulte de là que le capital du fonds ne pourra être considéré comme productif d'un revenu, qu'à partir du moment où les peuplements actuels seront abattus et remplacés par une nouvelle génération.

Cela posé, si nous considérons d'abord la partie qui renferme les bois exploitables, la valeur du fonds sera ce que vaut aujourd'hui le capital correspondant au revenu que donnera périodiquement cette partie de la forêt, lorsque les peuplements régénérés reviendront en tour d'exploitation. Ce revenu se composera :

$1^{\circ} \mathrm{Du}$ produit des coupes principales qui seront périodiquement exploitées à l'âge fixé par le terme de la nouvelle exploitabilité ;

$2^{\circ} \mathrm{Du}$ produit des coupes d'amélioration qui, si on les considère séparément, reviendront aussi périodiquement en tour d'exploitation, mais à des époques différentes des coupes principales.

Quant à la partie qui renferme les bois jeunes, la valeur du fonds se déterminera, comme nous l'avons fait dans le cas où le revenu est uniforme et périodique, en calculant ce que vaut actuellement le capital correspondant au revenu que donnera cette partie de la forêt après l'entière exploitation du bois qu'elle renferme. Ce revenu sera annuel ou périodique, continu ou discontinu, selon les combinaisons particulières de l'aménagement, et se com- 
posera des produits des coupes principales et des coupes d'amélioration.

Ces différentes sommes réunies feron! connaître la valeur brute de la forêto. Pour avoir sa valeur réelle, il nous reste à déduire de la valeur brute le capital de la dépense.

Dans le calcul de la dépense, on comprend les contributions, frais de garde, d'entretien, d'amélioration, etc. et, s'il y a lieu, la valeur des servitudes ou droits d'usage de toute espèce qui grèvent la forêt. Toutes ces dépenses seront capitalisées au même taux, et la différence entre le capital des recettes et le capital des dépenses exprimera la valeur neite, réelle et actuelle de la forêt.

\section{III.}

Cette manière d'estimer la valeur en fonds et superficie d'une forêt, considérée comme un capital placé en fonds de terre, s'applique à toutes celles que le défrichement ne peut atteindre.

Quant à la manière d'opérer en matière de cantonnement de droits d'usage, la marche à suivre, à l'égard des communes usagères dans les forêts de l'Etat, est tracée par un décret et une instruction administrative dont il ne nous appartient pas de discuter les dispositions. Disons seulement que l'intention du décret a été de favoriser l'exécution des cantonnements par la voie amiable, en concédant aux communes une part plus forte que celle à la- 
quelle elles pourraient prétendre d'après les règles tracées par la jurisprudence.

Mais lorsqu'il s'agit de cantonnements dans lesquels un particulier intervient, soit comme usager, soit comme propriétaire, les mèmes raisons n'existent plus pour obliger ou justifier la libéralité du propriétaire envers l'usager. Dans ce cas, l'opération du cantonnement consiste à détacher de la forêt grevée une partie telle que, si on voulait la vendre immédiatement, on pût en trouver un prix égal au droit capitalisé. Mais alors on se demande à quel taux on devra capitaliser la valeur de l'émolument usager? A cet égard, les tribunaux s'accordent assez généralement à prescrire l'emploi du taux légal, 5 p. 0/0, et plusieurs fois aussi ils ont prescrit l'emploi du même taux dans les calculs de capitalisation et d'escompte auxquels peut donner lieu l'estimation de la portion de forêt à abandonner en toute propriété à l'usager. Cette doctrine, qui nous parait équitable lorsqu'elle s'applique au cantonnement des droits de communes usagères dans les forêts de l'Etat, ne nous semble pas admissible dans le cas où un particulier intervient dans le cantonnement, soit comme usager, soit comme propriétaire. Nous pensons, au contraire, qu'après avoir capitalisé l'émolument usager au taux légal de $5 \mathrm{p} .0 / 0$, le propriétaire est autorisé par la loi elle-même, à employer le taux des placements en biens fonds dans les calculs relatifs à l'estimation de la portion de forêt à abandonner à l'usager, puisque la loi le force à se servir de cette 
monnaie pour se libérer, c'est-à-dire, à donner aux usagers, en propriété, une portion de forêt qu'il a achetée ou qu'il pourrait vendre au prix de son revenu capitalisé au taux ordinaire des placements en biens fonds dans la localité.

La question du taux à appliquer à la capitalisation de l'émolument usager et aux calculs relatifs à l'estimation du cantonnement est très-importante à faire décider, avant l'opération, par les parties lorsqu'il s'agit de cantonnements amiables, ou par les tribunaux dans le cas de cantonnements judiciaires. Il en est de même de la question des frais de garde et d'impôt. Cela fait, on détermine la partie de forêt dans laquelle on se propose d'asseoir le cantonnement, puis on estime les parcelles qu'elle renferme, en opérant séparément sur celles qui diffèrent entre elles par l'âge des bois ou par les conditions de la végétation, et en prenant pour base de l'estimation de chaque parcelle le revenu net qu'elle pourra fournir à l'âge correspondant au terme de son exploitabilité commerciale.

Telles sont les règles générales à suivre dans l'estimation de la valeur en fonds et superficie des propriétés dont la culture en nature de bois doit être conservée. Mais si, dans le problème d'estimation, on introduit la condition que le sol pourra être cultivé, au gré du propriétaire, soit en nature de bois, soit comme champ, comme pré, etc., l'estimateur devra rechercher d'abord la valeur en fonds et superficie de la propriété, comme si elle devait rester boisée; puis 
il calculera la plus value qui résulterait du défrichement de la forêt et de sa transformation en terre arable, en pré, etc., en tenant compte des frais que le changement de culture occasionnera. La comparaison de ces deux résultats fera connaître la vraie valeur de la propriété, et fera ressortir l'avantage ou la perte qui résulterait de sa transformation en terre arable. 


\title{
CHAPITRE TROISIÈME.
}

\author{
EXÉCUTION DES CALCULS.
}

\section{Article I.}

Observation générale.

On a vu au chapitre précédent que tous les calculs relatifs à l'estimation de la valeur des forêts en fonds et superficie se réduisent, en dernière analyse, à des opérations d'escompte et de capitalisation. Pour faciliter l'exécution de ces calculs, nous donnerons, à la fin de ce volume, des tarifs construits sur le modèle de ceux que Cotta a publiés pour le même objet. Au moyen de ces tarifs, dans la construction desquels l'unité a été prise partout pour base, tous les calculs relatifs au jeu des capitaux fonctionnant à intérêts c omposés peuvent se réduire à de simples multiplications.

Dans les formules génératrices de ces tarifs, nous avons désigné par $n$ le nombre d'années, par $t$ le taux de placement, et par $x$ l'inconnue ou la valeur de l'unité. 
Article II.

Formation et emploi du Tarif I.

Le Tarif I indique comment s'accroît l'unité, placée à intérêts composés, pendant un nombre d'années déterminé.

Dans la construction de ce tarif, on a supposé que les intérêts acquis au capital ne s'ajoutent à celui-ci qu'après l'entière expiration du nombre d'années que l'on considère.

La formule génératrice de ce tarif étant :

$$
x=(1+t)^{n},
$$

il s'ensuit, qu'en donnant successivement à $n$ la valeur $1,2,3,4$, etc., pour une valeur constante de $t$, on obtient pour la valeur correspondante de $x$ ce que devient l'unité, augmentée de ses intérêts composés, après une année, deux années, etc., de placement.

L'emploi de ce tarif est des plus simples. Veut-on savoir, par exemple, ce que devient un capital de $100 \mathrm{fr}$., placé à intérêts composés à 4 p. $0 / 0$ au bout de 20 ans? Il suffit de multiplier le capital placé, ou $100 \mathrm{fr}$., par le coefficient des tables correspondant à la $20^{\circ}$ année et au taux de $4 \mathrm{p}$. $0 / 0$, et l'on obtient $100 \times 2,191=219 \mathrm{fr} .10$.

Le même tarif sert encore à calculer les intérêts produits par un capital placé à intérêts composés pendant un temps déterminé, en retranchant la 
somme principale du produit capitalisé. C'est ainsi que l'on obtiendrait, pour les intérêts produits par un capital de 100 fr., iplacé à 4 p. $0 / 0$ pendant 20 ans, la somme de :

$100 \times 2,191-100=219,10-100=119$ fr. 10.

\section{Article III.}

Formation et emploi du Tarif II.

Le Tarif II indique la valeur actuelle de l'unité payable après un nombre d'années déterminé. C'est donc un tarif d'escompte dans la construction duquel l'escompte a été pris en dedans.

La formule génératrice de ce tarif est la suivante :

$$
x=\frac{1}{(1+i)^{n}}
$$

Le Tarif Il sert spécialement à déterminer la valeur actuelle d'une somme à toucher après un nombre d'années déterminé, en multipliant cette somme par le coefficient des tables correspondant au nombre d'années et au taux d'intérêt que l'on considère.

Par exemple, veut-on savoir ce que vaut aujourd'hui une somme de $500 \mathrm{fr}$., payable dans 12 ans révolus, dans l'hypothèse d'un placement à $5 \mathrm{p} .0 / 0$ ? Il suffit de multiplier la somme à toucher par le coefficient porté dans la colonne intitulée 5 p. $0 / 0$, en regard de la $12^{\circ}$ année, et l'on trouve :

$$
500 \times 0,557=278 \text { fr. } 50 \text { cent. }
$$


CalCUls.

La différence entre la somme à toucher et sa valeur actuelle représente ce qu'on nomme l'escompte de cette somme. Dans l'exemple précédent l'escompte serait de $500-278,50=221$ fr. 50 .

Autre exemple. - Un bois âgé de 15 ans est susceptible de fournir un produit net de $6000 \mathrm{fr}$. à 25 ans, terme de son exploitation; que vaut aujourd'hui la superficie de ce bois, en admettant que le taux des placements en fonds de terre dans la localité soit de $3 \mathrm{p} .0 / 0$ ?

$\mathrm{La}$ réponse à cette question s'obtiendra à l'aide du Tarif II, en escomptant à 3 p. $0 / 0$ la somme de $6000 \mathrm{fr}$. à toucher dans 10 ans; ce sera $6000 \times$ $0,744=4464 \mathrm{fr}$.

Article IV.

Formation et emploi du Tarif III.

Le Tarif III est un tarif de capitalisation. Il donne le capital correspondant à un revenu égal à l'unité payable à des intervalles égaux, par exemple tous les 5 ans, tous les 10 ans, etc., mais à la condition que le revenu ne commencera à être perçu qu'à $l^{\prime}$ expiration de la $5^{\mathrm{e}}$, de la $10^{\circ}$ année, etc.

Ce tarif a été formé au moyen de la formule :

$$
x=\frac{1}{(1+l)_{n}-1}
$$

On se sert de ce tarif pour calculer la valeur actuelle 
du capital équivalent à un revenu perpétuel et périodique, en multipliant ce revenu par le coefficient correspondant au nombre d'années de la période et au taux de placement.

Exemple. - Veut-on savoir quelle est la valeur d'un bois qui vient d'être exploité, et qui produit tous les 15 ans un revenu de $500 \mathrm{f}$., en supposant que le taux des placements en fonds de terre dans la localité soit de 3 p. $0 / 0$ ?

On cherche dans le Tarif III le coefficient correspondant à la $15^{\mathrm{e}}$ année et au taux de $3 \mathrm{p} .0 / 0$; on multiplie le revenu à toucher tous les 15 ans par ce coefficient, et l'on obtient pour la valeur cherchée :

$$
500 \times 1,792=896 \mathrm{fr} .
$$

On voit, par cet exemple, que le Tarif III sert spécialement à calculer la valeur actuelle $d u$ fonds de terre dans l'estimation des forêts en fonds en superficie.

\section{Article V.}

Formation et emploi du Tarif IV.

Le Tarif IV est un tarif de capitalisation. Il donne le capital équivalent à un revenu annuel et continu, égal à l'unité, que l'on ne commence à toucher qu'après un temps déterminé.

La formule génératrice de ce tarif est la suivante:

$$
x=\frac{1}{t(1+t)^{n-1}}
$$


On se sert du Tarif IV pour obtenir la valeur actuelle des capitaux pouvant produire à perpétuité une rente annuelle dont on ne jouira qu'après un temps déterminé, en multipliant ce revenu par le coefficient correspondant à l'année dans laquelle on commencera à le percevoir et au taux d'intérêt admis pour le placement des capitaux de même espèce.

Exemple. - Quelle est la valeur actuelle d'un bois susceptible de produire annuellement et à perpétuité un revenu net de $500 \mathrm{fr}$., et situé dans une contrée où le taux des placements en fonds de terre est de $3 \mathrm{p}, 0 / 0$ ?

Si l'acheteur doit entrer en jouissance immédirtement, la valeur de la propriété s'obtiendra en multipliant le revenu net par le denier 33,333, c'est-àdire, par le coefficient du tarif correspondant à la première année et au taux de 3 p. $0 / 0$, soit :

$$
500 \times 33,333=16666 \text { fr. } 50 .
$$

Mais, si l'acheteur ne doit commencer à percevoir le revenu que dans cinq ans révolus par exemple, la valeur cherchée s'obtiendra en multipliant le revenu par le coefficient correspondant à la 5e année et au taux de 3 p. 0/0. Ce sera....

$$
500 \times 29,616=14808 \mathrm{fr} .
$$

La vérification de ce dernier résultat peut se faire à l'aide du Tarif II en escomptant, pour 4 ans, la somme 16666 fr. 50 que l'on obtient en multi- 
pliant le revenu par le denier de placement. Nous disons - pour 4 ans - parce que, si l'acheteur doit toucher le premier revenu après cinq ans révolus, il est clair qu'on doit le considérer comme ayant pris possession de la propriété au début de la $5^{\circ}$ année, ou à l'expiration de la $4^{\circ}$. En effet, si on effectue les calculs on obtient :

$$
16666,50 \times 0,888=14800 \mathrm{fr} .
$$

résultat qui ne diffère du précédent que par le chiffre des unités.

\section{Article VI.}

Formation et emploi du Tarif V.

Le Tarif $\mathrm{V}$ donne la valeur actuelle d'un revenu annuel, égal à l'unité, à percevoir pendant un nombre d'années déterminé. C'est, par conséquent, un tarif d'escompte, comme le Tarif II au moyen duquel on trouverait cette valeur en escomptant le revenu successivement pour chaque année où il sera perçu, ou, ce qui revient au même, en multipliant le revenu par la somme des facteurs que l'on aurait employés isolément.

Le Tarif $\mathrm{V}$ a précisément pour objet de donner, pour chaque année, la somme des facteurs du Tarif II depuis la première année jusques y compris la dernière que l'on considère. Les facteurs du Tarif II formant une progression géométrique décroissante 
dont le premier terme et la raison sont égaux à $\frac{1}{1+t}$, la somme des $n$ premiers termes de cette progression sera égale à :

$$
\frac{\frac{1}{1+t}-\left(\frac{1}{1+t}\right)^{n+1}}{1-\frac{1}{1+t}}
$$

C'est en effet la formule génératrice du Tarif $\mathrm{V}$, laquelle peut encore s'écrire sous la forme :

$$
x=\frac{1}{t}\left(1-\frac{1}{(1+t)^{n}}\right)
$$

Ce tarif s'emploie d'une manière analogue aux précédents.

Exemple. - Le propriétaire d'une forêt met en vente la superficie de cinq coupes à exploiter successivement en cinq années. Chaque coupe devant fournir un produit net de $1500 \mathrm{fr}$., on demande ce que vaut aujourd'hui la superficie des cinq coupes pour un acheteur qui serait disposé à en avancer le prix moyennant un intérêt de 5 p. $0 / 0$ ?

Il y a deux cas à considérer :

Si le premier revenu doit être touché à la fin de la première année, la valeur actuelle de la superficie s'obtiendra en multipliant le revenu par le coefficient correspondant à la $5^{\mathrm{e}}$ année, soit :

$$
1500 \times 4,329=6493 \text { fr. } 30 \text {. }
$$

Mais si le premier revenu ne doit être perçu 
DE L'ESTIMATION EN FONDS ET SUPERFICIE.

qu'après un nombre d'années déterminé, le coefficient par lequel on devra multiplier le revenu pour avoir le capital cherché s'obtiendra en retranchant le coefficient correspondant à l'année qui précède celle où le revenu sera perçu pour la première fois, du coefficient de l'année qui précède celle dans laquelle le revenu sera touché pour la dernière fois. Si donc on suppose, dans l'exemple précédent, que le premier revenu ne doive être perçu qu'à la fin de la $7^{\circledR}$ année, la valeur actuelle de la superficie des cinq coupes à vendre sera égale au revenu multiplié par la différence entre les coefficients de la $11^{\text {e }}$ et de la $6^{\mathrm{e}}$ année, soit :

$$
1500 \times(8,306-5,076)=4848 \mathrm{fr} .
$$




\title{
CHAPITRE QUATRIÈME.
}

\author{
PROBLÈMES SUR L'ESTIMATION DES FORÈTS EN FONDS \\ ET SOPERFICIE.
}

PR OBLĖME No 1 .

VENTE DE SUPERFICIE.

Un particulier possède une forêt de 300 hectares, régulièrement exploitée en taillis simple à une révolution de 20 ans, et susceptible d'un produit net de 620 fr. par hectare exploitable.

On met en vente, au comptant, la superficie tout entière, à la condition qu'elle sera exploitée en 20 coupes d'égale contenance. Mais depuis trois ans il y a eu anticipation de jouissance, et le propriétaire a fait exploiter deux coupes au lieu d'une. On veut revenir aux prescriptions de l'aménagement, c'est-à-dire, exploiter par $20^{\circ}$ de surface et chaque coupe à l'âge minimum de 20 ans.

On demande ce que vaut actuellement la superficie pour un spéculateur qui veut trouver un emploi de ses fonds à 5 p. 0/0, et réaliser sur l'exploitation un bénéfice de $10 \mathrm{p.} 0 / 0$.

On admet, qu'à partir de 20 ans, la valeur sur pied des coupes exploitables s'accroît comme un capital placé à 3 p. 0/0.

\section{SOLUTION.}

D'après l'énoncé, la première coupe ne sera exploitée que dans 3 ans, et le produit en argent 
qu'elle donnera ne pourra être perçu qu'à la fin de la $3^{e}$ année. Chaque coupe comprendra 15 hectares. Les 15 premières seront exploitées à 20 ans, la $16^{\circ}$ et la $17^{\mathrm{e}}$ à 21 ans, la $18^{\mathrm{e}}$ et la $19^{\mathrm{e}}$ à 22 ans, et la $20^{\circ}$ à 23 ans.

Les 15 premières coupes donneront un revenu annuel de $15 \times 620=9300 \mathrm{fr}$., qui durera $1 \mathrm{~J}$ ans et ne commencera à être perçu qu'à la fin de la $3^{e}$ année. Cette somme escomptée par le Tarif $\mathrm{V}$, au taux de 5 p. 0/0, donne pour la valeur actuelle des 15 premières coupes :

$$
\text { (1). . } 9300(11,274-1,859)=87559 \mathrm{fr} .
$$

Les $16^{\mathrm{e}}$ et $17^{\mathrm{e}}$ coupes ne seront exploitées qu'à 21 ans. La valeur de ces coupes devant s'accroître, de la $20^{\circ}$ à la 21 année, comme un capital placé à $3 \mathrm{p} .0 / 0$, chacune d'elles fournira (Tarif I) un revenu égal à $9300 \times 1,03=9$ s̆79 fr. Cette somme escomptée à 5 p. $0 / 0$, par le Tarif $\mathrm{V}$, comme un revenu qui durera- 2 ans et ne commencera à être perçu qu'à la fin de la $19^{\circ}$ année, donne pour la valeur actuelle des $16^{\mathrm{e}}$ et $17^{\mathrm{e}}$ coupes :

$$
\text { (2) . . } 9579(12,462-11,689)=740 ว \mathrm{fr} \text {. }
$$

Les $18^{\circ}$ et $19^{\circ}$ coupes ne seront exploitées qu'à 22 ans. La valeur de ces coupes devant s'accroître, de la $20^{\circ}$ à la $22^{\mathrm{e}}$ année, comme un capital placé à 3 p. 0/0, chacune d'elles fournira, d'après le Tarif $I$, un revenu égal à $9300 \times 1,061=9867 \mathrm{fr}$. 30 . Cette somme escomptée à 5 p. $0 / 0$, par le Tarif $\mathrm{V}$, 
comme un revenu qui durera 2 ans et ne commencera à être perçue qu'à la fin de la $21^{\circ}$ année, donne pour la valeur actuelle des $18^{\circ}$ et $19^{\circ}$ coupes :

(3) . . 9867,30 (13,163-12,462) $=6917 \mathrm{fr}$.

La $20^{\circ}$ coupe ne sera exploitée qu'à 23 ans. La valeur de cette coupe devant s'accroître, de la $20^{e}$ à la $23^{e}$ année, comme un capital placé à 3 p. 0/0, elle fournira, d'après le Tarif $\mathrm{I}$, un revenu égal à $9300 \times 1,093=10164$ fr. 90 . Cette somme escomptée à 5 p. 0/0, par le Tarif II, donne pour la valeur actuelle de la $20^{\circ}$ coupe :

$$
\text { (4). . 10164,90×0,326=3314fr. }
$$

Faisant la somme de ces quatre résultats, on a pour la valeur actuelle de la superficie :

$87559+7405+6917+3314=105195 \mathrm{fr}$.

Mais l'acheteur veut en outre réaliser sur les produits de l'exploitation un bénéfice de 10 p. 0/0, dont la valeur actuelle serait de $10519 \mathrm{fr}$.; si donc on retranche cette valeur de la précédente, la différence exprimera la somme que l'acquéreur peut offrir aujourd'hui de la superficie, soit :

$$
105195-10519=94676 \text { fr. }
$$

PROBLÈME NO 2.

VENTE DE FONDS ET SUPERFICIE.

Une forêt de 300 hectares, exploitée en taillis simple et ré- 
gulièrement aménagée à une révolution de 25 ans, est susceptible d'un revenu annuel de $800 \mathrm{fr}$. par hectare exploitable.

Les frais annuels de garde et d'impôt s'élèvent à 5 fr. par hectare.

On demande :

10 Quelle est la valeur en fonds et superficie de cette forêt, pour un capitaliste qui veut trouver un placement de son argent à 4 p. $0 / 0$ ?

$2^{\circ}$ Quelle serait la valeur en fonds et superficie des 5 coupes âgées de 21 à 25 ans, dans le cas où ces coupes devraient continuer à être exploitées à 25 ans?

\section{SOLUTION.}

I.

Le revenu étant annuel et constant, la valeur en fonds et superficic de toute la forêt s'obtiendra en multipliant le revenu brut par le facteur de la première année du Tarif IV correspondant au taux de 4 p. $0 / 0$, et en déduisant du résultat le capital qui pourrait fournir annuellement, en intérêts seulement, une somme équivalente aux frais.

L'étendue de la coupe annuelle est de $\frac{300}{25}=12$ hectares dont le produit est de $9600 \mathrm{fr}$. Le Tarif IV donne pour le capital correspondant à ce revenu :

$$
9600 \times 25=240000 \mathrm{fr} .
$$

Les frais annuels sont de $300 \times \tilde{5}=1500 \mathrm{fr}$. Le Tarif IV donne pour le capital équivalent aux frais annuels de garde et d'impôt :

$$
1500 \times 25=37500 \mathrm{fr} .
$$


Retranchant ce second produit du premier, on a pour la valeur en fonds et superficie de la forêt, au taux de $4 \mathrm{p}$. 0/0 :

$$
240000-37500=202500 \text { fr. }
$$

II.

Dans le cas où les 5 coupes âgées de 21 à' 25 ans devraient continuer à être exploitées à 25 ans, elles fourniraient d'abord 弓̆ revenus successifs égaux à $9600 \mathrm{fr}$.; puis il y aurait interruption de jouissance pendant 20 ans, après lesquels on percevrait de nouveau 5 revenus successifs égaux à 9600 fr., et ainsi de suite indéfiniment.

Dans ces conditions, si on escompte, au moyen du Tarif V, les cinq revenus successif́s à toucher dans les 5 premières années de la première révolution de 25 ans, le résultat exprimera ce que vaut aujourd'hui la superficie existante, et ce que vaudront les 5 coupes au début de chaque période. Dès lors, pour avoir la valeur du fonds, il ne restera plus qu'à capitaliser ce résultal, comme une rente à toucher périodiquement tous les 25 ans, au moyen du Tarif III.

En opérant de cette manière, on trouve que les cinq revenus à toucher successivement valent réunis, au début de chaque période :

Superficie (Tarif V), $9600 \times 4,452=42739 \mathrm{fr}$.

Le Tarif III donne pour le capital équivalent à la valeur du fonds : 
Fonds : $42739 \times 0,600=25643 \mathrm{fr}$.

D'où, en faisant la somme, on obtient pour la valeur en fonds et superficie des cinq coupes âgées de 21 à 25 ans :

$$
42739+25643=68382 \mathrm{fr} .
$$

Et en retranchant le capital des frais, ou

$$
\begin{aligned}
& \qquad \begin{aligned}
12 \times 5 \times 25 & =1500 \mathrm{fr} . \\
\text { Il reste pour valeur nette : } & \\
68382-1500 & =66882 \mathrm{fr} .
\end{aligned}
\end{aligned}
$$

PROBLEME No 3.

ÉCHANGE .

Par suite de convenances personnelles, deux proprietaires veulent échanger leurs forêts.

Le propriétaire $A$ possède 200 hectares de taillis sous futaie, régulièrement aménagés à 20 ans, et susceptibles d'un produit annuel de $1100 \mathrm{fr}$. par hectare exploitable, façon déduite.

Le propriétaire $B$ possède 300 hectares de taillis simple, régulièrement aménagés à 25 ans, et susceptibles de fournir un revenu annuel de 800 fr. par hectare exploitable, façon déduite.

Examen fait des conditions dans lesquelles les deux forêts végètent, on constate :

$1^{\circ}$ Que le taux des placements en fonds de terre, dans la localité où sont situées les deux propriétés, est de $3 \mathrm{p} .0 / 0$.

$2^{\circ}$ Qu'il n'y a aucun intérêt à changer le régime auquel elles sont respectivement soumises, et que, par conséquent, la première devra continuer à être exploitée en taillis composé, la seconde en taillis simple; 
$3^{\circ}$ Que le plan de balivage appliqué à la première répond aux conditions de l'exploitabilité commerciale, et, par conséquent, assure la production du revenu pécuniaire le plus avantageux ;

$4^{\circ}$ Que la deuxième forêt pouvant fournir, par hectare exploitable à 20 ans, un revenu de $600 \mathrm{fr}$., tous frais déduits, il y a lieu de réduire à 20 ans la révolution à laquelle elle est actuellement soumise;

$5^{\circ}$ Que pour les deux forêts, les frais annuels de garde et d'impôt sont de $\breve{J}$ fr. par hectare.

On demande quelle est la valeur en fonds et superficie des deux propriétés, et quelle sera la soulte à payer par suite de l'échange.

\section{SOLUTION.}

La forêt $A$, devant continuer à être traitée de la même manière que par le passé, fournira annuellement un revenu de $1100 \mathrm{fr}$. par hectare exploitable, ou de $1100 \times 8=8800$ fr. pour chaque coupe; et en déduisant les frais de garde $8800-1000=$ $7800 \mathrm{fr}$.

Ce revenu capitalisé par le Tarif IV donne pour la valeur en fonds et superficie de la forêt entière une somme de :

$$
\text { (1) } \ldots 7800 \times 33,333=259997 \mathrm{fr} .
$$

I $L$ forêt $B$, devant être ramenée à une révolution de 20 ans, peut être considérée comme divisée en deux parties : la première comprenant les coupes âgées de 1 à 20 ans, la seconde renfermant les coupes âgées de 21 à 25 ans.

La première partie comprenant 240 heclares, 
fournira annuellement une coupe de 12 hectares laquelle produira un revenu de :

$$
12 \times 600=7200 \mathrm{fr} .
$$

Ce révenu capitalisé par le Tarif IV donne pour la valeur en fonds et superficie de la première partie :

$$
\text { (2) } \ldots 7200 \times 33,333=239997 \mathrm{fr} \text {. }
$$

La deuxième partie de la forêt $B$ comprend 60 hectares ou cinq coupes dont la superficie est immédiatement réalisable. Mais si, pour faciliter l'écoulement des produits à un prix convenable, on admet que ces coupes devront être exploitées en cinq ans, une première fois à 25 ans, et plus tard à la révolution de 20 ans, chacune d'elles fournira un revenu de $12 \times 800=9600 \mathrm{fr}$., lequel commencera à être perçu à la fin de la première année et durera $ّ$ ans. Le Tarif $\mathrm{V}$ donnera pour la valeur actuelle de la superficie de ces 5 coupes :

$$
9600 \times 4,580=43968 \mathrm{fr} .
$$

Reste à calculer la valeur du fonds. Or, le fonds doit produire à 20 ans un revenu de $600 \mathrm{fr}$. par hectare ou de $600 \times 12=7200$ fr. par coupe. Ce revenu sera périodique et durera cinq ans. Sa valeur au début de chaque période sera, d'après le Tarif $\mathrm{V}$, égale à $7200 \times 4,580=32976 \mathrm{fr}$, , et le capital équivalent à ce revenu, calculé d'après le Tarif III, sera :

$$
32976 \times 1,240=40890 \mathrm{fr} .
$$


Faisant la somme des deux derniers produits, on a pour la valeur en fonds et superficie de la seconde partie de la forêt $B$ :

$$
\text { (3) } \ldots 43968+40890=84858 \mathrm{fr} \text {. }
$$

Ajoutant ce dernier résultat à celui qui exprime (2) la valeur de la première partie, on obtient pour la valeur en fonds et superficie de la forêt $B$ :

$$
\text { (4) } \ldots 84868+239997=324855 \mathrm{fr} \text {. }
$$

Mais de cette somme il faut retrancher le capital des frais de garde et d'impôt, que l'on obtient au moyen du Tarif IV et qui représente une valeur de $300 \times 5 \times 33,333=50000 \mathrm{fr}$. ; et l'on a pour la valeur nette en fonds et superficie de la forêt $B$ :

(5) $\ldots 324855-50000=274855 \mathrm{fr}$.

Faisant la différence entre ce résultat et celui qui exprime (1) la valeur nette en fonds et superficie de la forêt $A$, on trouve que le propriétaire $B$ aura à payer au propriétaire $A$ une soulte de :

$$
\text { (6) } \ldots 274855-239997=34858 \mathrm{fr} \text {. }
$$

\section{PROBLÈME No 4 .}

PARTAGE.

On veut partager en trois parties d'égale valeur une forêt de 300 hectares, régulièrement aménagée en taillis composé à la révolution de 30 ans, à la condition d'affecter à chacun des co-partageants une portion dans laquelle les âges seront gradués de proche en proche. 
Examen fait des conditions de végétation et de consistance des peuplements de chaque coupe, on constate :

$1^{\circ}$ Que les coupes âgées de 3 et de 4 ans fourniront un produit de 1800 fr. par hectare exploitable à 30 ans;

$2^{\circ}$ Que les coupes âgées de 15 et de 19 ans produiront $1100 \mathrm{fr}$. par hectare à 30 ans;

$3^{\circ}$ Que les coupes âgées de 26 et de 29 ans donneront un produit de $1200 \mathrm{fr}$. par hectare à 30 ans;

$4^{\circ}$ Que toutes les autres coupes produiront à 30 ans un revenu de 1500 fr. par hectare;

$5^{\circ}$ Qu'il n'y a pas lieu de changer le régime, la révolution et le plan de balivage précédemment suivis.

On stipule que les co-partageants règleront entre eux l'inégalité des frais qui incomberontà chacun d'eux, par suite de l'inégalité des contenances qui leur seront attribuées.

Le taux des placements en fonds de terre dans la localité, est de 3 p. $0 / 0$.

\section{SOLUTION.}

I.

La forêt contenant 300 hectares, chaque coupe comprend une étendue de 10 hectares.

Les coupes âgées de 1 an et de 2 ans donneront un produit de $1500 \mathrm{fr}$. par hectare à 30 ans, soit pour chaque coupe un produit de $15000 \mathrm{fr}$. Ce revenu durera deux ans et commencera à être perçu à la fin de la $29^{\circ}$ année. Ce revenu, escompté par le Tarif V, exprimera ce que vaut aujourd'hui la superficie des deux coupes âgées de 1 et de 2 ans, soit :

$$
15000(19,600-18,764)=12540 \mathrm{fr} .
$$


Mais, après la première exploitation, le revenu rentrera périodiquement tous les 30 ans. Le capital équivalent à ce revenu s'obtiendra à l'aide du Tarif III, et représentera la valeur actuelle du fonds des deux coupes âgées de 1 an et de 2 ans. Ce sera:

$$
12540 \times 0,701=8790 \mathrm{fr} .
$$

En additionnant ces deux résultats, on a pour la valeur actuelle en fonds et superficie des deux coupes âgées de 1 an et de 2 ans :

$$
\text { (1) } \ldots 12540+8790=21330 \mathrm{fr} \text {. }
$$

Les coupes âgées de 3 et 4 ans produiront un revenu de $18000 \mathrm{fr}$., qui commencera à être perçu dans 27 ans et durera 2 ans. Leur valeur actuelle en fonds et superficie sera :

superficie (T. V) $18000(18,764-17,877)=15966 \mathrm{f}$. fonds (Tarif III) $15966 \times 0,701=\ldots .11192$

$$
\text { (2)... Total... } 27158 \mathrm{f} \text {. }
$$

Les 10 coupes âgées de 5 à 14 ans fourniront chacune un produit de $15000 \mathrm{fr}$., qui sera perȩu pour la première fois dans 17 ans, durera 10 ans, et reviendra ensuite périodiquement tous les 30 ans. Leur valeur actuelle en fonds et superficie sera :

superficie(T.V) $15000(17,877-12,561)=79740 \mathrm{f}$. fonds (Tarif III) $79740 \times 0,701=\ldots .55897$

$$
\text { (3) ... Total ... } \overline{135637 \mathrm{f}} \text {. }
$$

La coupe âgée de 15 ans fournira un produit de 
$11000 \mathrm{fr}$., qui sera perçu pour la première fois dans 16 ans, et reviendra ensuite périodiquement tous les 30 ans. La valeur de la superficie s'obtiendra par le Tarif II, celle du fonds par le Tarif III et l'on aura pour la valeur actuelle en fonds et superficie de la coupe âgée de 15 ans :

superficie (Tarif II) $\ldots \quad 11000 \times 0,623=6853 \mathrm{f}$. fonds (Tarif III) .... $6853 \times 0,701=4804$

(4)...Total... $\overline{11657 \mathrm{f}}$.

Les coupes âgées de 16,17 et 18 ans fourniront chacune un produit de 15000 fr., qui commencera à être perçu dans 13 ans et durera 3 ans. La valeur actuelle en fonds et superficie de ces trois coupes sera :

superficie (T.V) $15000(11,938-9,954)=29760 \mathrm{f}$. fonds (Tarif III) $29760 \times 0,701=\ldots .20842$

(5) ... Total ... $50602 \mathrm{f}$.

La coupe âgée de 19 ans produira un revenu de $11000 \mathrm{fr}$., qui sera perçu une première fois dans 12 ans, et reviendra ensuite périodiquement tous les 30 ans. La valeur de la superficie s'obtiendra par le Tarif II, celle du fonds par le Tarif III, et l'on aura pour la valeur actuelle en fonds et superficie de cette coupe :

superficie (Tarif II) $\ldots 11000 \times 0,701=7711 \mathrm{fr}$. fonds (Tarif III)..... $7711 \times 0,701=5405$

(6) ... Total ... $13116 \mathrm{fr}$. 
Les 6 coupes âgées de 20 à 2 ว̆ ans fourniront chacune un produit de 15000 fr., qui commencera à être perçu dans 6 ans et durera 6 ans. Leur valeur actuelle en fonds et superficie sera :

superficie (T.V) $15000(9,253-4,580)=70095 \mathrm{fr}$. fonds (Tarif III) $70095 \times 0,701=\ldots \ldots 49136$

(7) ... Total .... $\overline{119231 \mathrm{fr}}$.

La coupe âgée de 26 ans produira un revenu de 12000 fr., qui sera perçu une première fois dans 5 ans et reviendra ensuite périodiquement tous les 30 ans. La valeur actuelle en fonds et superficie de cette coupe sera :

superficie (Tarif II) . $12000 \times 0,863=10356 \mathrm{fr}$. fonds (Tarif III). . . $10356 \times 0,701=7260$

$$
\text { (8)... Total ... } \overline{17616 \mathrm{fr} \text {. }}
$$

Les deux coupes âgées de 27 et 28 ans produiront chacune un revenu de 15000 fr., qui sera perçu pour la première fois dans 3 ans et durera 2 ans. Leur valeur actuelle en fonds et superficie sera :

superficie (T. V) $15000(3,717-1,913)=27060 \mathrm{f}$. fonds (Tarif III) $27060 \times 0,701=\ldots \ldots 18969$

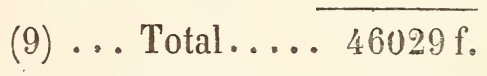

La coupe âgée de 29 ans produira un revenu de 12000 fr., qui sera perçu une première fois dans 2 ans, et reviendra ensuite périodiquement tous les 30 ans. Sa valeur actuelle en fonds et superficie sera : 
superficie $($ Tarif II) $\ldots 12000 \times 0,943=11316 \mathrm{f}$. fonds (Tarif III) .... $11316 \times 0,701=7932$

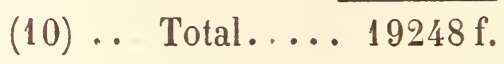

La coupe âgée de 30 ans produira un revenu de 15000 fr., qui sera perçu une première fois dans un an, et reviendra ensuite périodiquement tous les 30 ans. Sa valeur actuelle en fonds et superficie sera :

superficie (Tarif II) $\ldots 15000 \times 0,971=14565 \mathrm{f}$. fonds (Tarif III) .... 14565 $\times 0,701=10210$

$$
\text { (11). . Total.... } 24775 \mathrm{f} \text {. }
$$

En additionnant les sommes qui représentent la valeur actuelle des 30 coupes, le total exprimera ce que vaut aujourd'hui en fonds et superficie la forêt à partager. Ce total étant de $486409 \mathrm{fr}$., il ne reste plus qu'à diviser la forêt en trois parties dont chacune représente actuellement une valeur de :

$$
\frac{486409}{5}=162136 \text { fr., }
$$

et soit composée de peuplements d'âges gradués de proche en proche.

II.

1ег Lот.

Si l'on affecte au premier lot les bois les plus jeunes, ce lot comprendra : 
PROBLEMES.

$20 \mathrm{~h}$. de bois de 1 an et 2 ans, valant............ $21330 \mathrm{f}$.

20 h. de bois de 3 et 4 ans.... 27158

$80 \mathrm{~h} . \quad$ id. $\quad 5$ à 12 ans..... 105225

5 h. 6226 id. 13 ans....... 8417

$\overline{125 \text { h. } 6226}$................ $\overline{162130 \mathrm{f} \text {. }}$

2 Lот.

Le $2^{\mathrm{e}}$ lot comprendra :

$4 \mathrm{~h} .3774$ de bois de 13 ans....... $6503 \mathrm{f}$. $10 \mathrm{~h} . \quad$ id. $\quad 14$ ans........ 15436 10 h. $\quad$ id. $\quad 15$ ans....... 11657 $30 \mathrm{~h} . \quad$ id. 16 à 18 ans.... 50622 $10 \mathrm{~h} . \quad$ id. 19 ans....... 13116 $30 \mathrm{~h} . \quad$ id. 20 à 22 ans.... 5697 J 3 h. 8633 id. 23 ans....... 7777 98 h. $2407 \ldots \ldots \ldots \ldots \ldots \ldots . .$.

зе Lот.

Le $3^{\mathrm{e}}$ lot comprendra :

6 h. 1367 de bois de 23 ans....... 12353 f. $20 \mathrm{~h} . \quad$ id. 24 et 25 ans... 42100 $10 \mathrm{~h} . \quad$ id. 26 ans....... 17616 20 h. $\quad$ id. 27 et 28 ans.... 46029 $10 \mathrm{~h} . \quad$ id. 29 ans....... 19248 $10 \mathrm{~h} . \quad$ id. $\quad 30$ ans....... 24775 76 h. $1367 \ldots \ldots \ldots \ldots \ldots \ldots . .162121 \mathrm{f}$. 


\section{PROBLÈME No}

Vente dR Fonds et SUPERficie, aVEc faculté De défricher.

Le parc attenant au château de..... est mis en vente, avec faculté de défricher. Il contient 60 hectares, uniformément peuplés d'une futaie de chêne âgée de 120 ans.

Le volume actuel de la superficie est de 27300 mètres cubes, dont :

2/5 en bois de service, valant $40 \mathrm{f}$. le m. c. sur pied.

$1 / 5$ en bois de travail, - 30

2/5 en bois de feu, - 5

On demande quelle est la valeur de cette propriété pour un capitaliste qui veut placer ses fonds à $4 \mathrm{p} .0 / 0$, et réaliser un bénéfice de $10 \mathrm{p}$. 0/0 sur la vente des bois qui forment la superficie actuelle.

On admet :

$1^{\circ}$ Que, pour faciliter la vente des produits, l'exploitation de la superficie devra avoir lieu en cinq ans et par $5^{\mathrm{e}}$ de surface;

$2^{\circ}$ Que l'accroissement annuel des bois qui seront exploités de la $121^{\mathrm{e}}$ à la $125^{\circ}$ année sera égal à l'accroissement moyen passé du même matériel à 120 ans;

$3^{\circ}$ Que la conversion de cette futaie en taillis simple assurerait un revenu de 700 fr. par hectare exploitable à 20 ans;

$4^{\circ}$ Qu'en cas de défrichement, chaque partie pourrait être affermée, un an après le défrichement, au prix de 50 fr. par hectare;

$5^{\circ}$ Que la valeur des souches suffira pour couvrir les frais du défrichement;

$6^{\circ}$ Que les frais annuels de garde, d'impôt, etc., seraient,

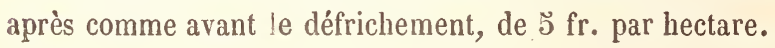


PROBLÈMES.

SOLUTION.

I.

Il s'agit d'examiner d'abord s'il y a lieu de défricher. L'exploitation de la superficie devant avoir lieu en cinq ans et par $5^{\circ}$ de surface, la coupe de la $1^{\text {ro }}$ année fournira

$$
\frac{27300}{5}=5460^{\text {m.c. }}
$$

dont $2 / 5=2184 \quad$ à $\quad 40 \mathrm{fr} .=87360 \mathrm{fr}$.

$1 / 5=1092$ à $30=32760$

$2 / \tilde{a}=2184$ à $\quad \breve{s}=10920$

Totaux.... $\overline{5460^{\mathrm{m}} . \mathrm{c}}$

$131040 \mathrm{fr}$.

La seconde année on réalisera le même produit augmenté de la valeur d'un accroissement moyen à 120 ans, soit :

$$
131040+\frac{131040}{120} 131040+1092=132132 \mathrm{fr} .
$$

De même on réalisera :

dans la $3^{\circ}$ année $131040+1092 \times 2=133224 \mathrm{f}$. dans la $4^{\circ}$ id. $\quad 131040+1092 \times 3=134316$ dans la $5^{\circ}$ id. $\quad 131040+1092 \times 4=135408$

Le Tarif II donne pour la valeur actuelle de la superficie de ces cinq coupes :

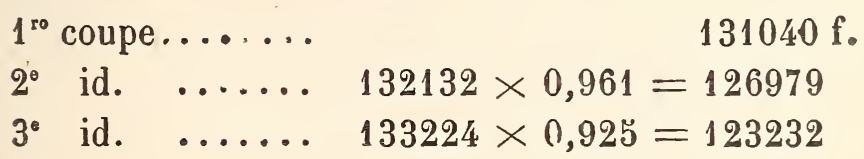


$4^{8}$ coupe $\ldots \ldots \ldots 134316 \times 0,889=119407$

$5^{\mathrm{e}} \quad$ id. $\quad \ldots \ldots \ldots 135408 \times 0,855=115774$

Total..... 616432

Dans ces conditions, la valeur du fonds serait, d'après le Tarif III, de $616332 \times 0,009=5547$ fr., plus le capital équivalent au produit des coupes d'éclaircie à pratiquer dans le cours de la révolution. Or, en admettant que chacune de ces opérations portât sur la totalité des peuplements, et pût fournir tous les 20 ans un produit de 300 fr. par hectare, ou de 18000 fr. pour les 60 hectares, le capital équivalent à ce revenu, calculé par le Tarif III, serait de $18000 \times 0,839=15102 \mathrm{fr}$, et l'on aurait pour la valeur du fonds :

(1) $5547+15102=20649 \mathrm{fr}$.

Mais si l'on convertissait la futaie en un taillis simple exploitable à 20 ans, le revenu de chaque coupe serait de $700 \times 12=8400 \mathrm{fr}$. Ce revenu serait touché pour la $1^{\text {re }}$ fois dans 20 ans et durerait 5 ans, puis il y aurait interruption de jouissance pendant 15 ans après lesquels on percevrait le même revenu pendant כ̌ ans, et ainsi de suite. En calculant la valeur, au début de chaque période, des cinq revenus successifs à toucher périodiquement, et cherchant le capital correspondant à cette somme considérée comme une rente périodique à toucher tous les 20 ans, le résultat exprimera la valeur actuelle du fonds. 
PROBLÈMES.

Au moyen du Tarif $\mathrm{V}$ on trouve que les cinq revenus à toucher successivement ont, au début de chaque période de 20 ans, une valeur de $8400 \times$ $4452=37397 \mathrm{fr}$. D'ailleurs, le Tarif III donne pour le capital correspondant à ce revenu :

(2) Valeur du fonds $=37397 \times 0,840=31413 \mathrm{f}$.

Si maintenant on compare ce résultat avec celui qui exprime la valeur du fonds (1), dans le cas où l'on continuerait à exploiter en futaie à une révolution de 120 ans, on voit qu'il y a intérêt pour le propriétaire à convertir la futaie en un taillis simple exploitable à 20 ans.

Mais il reste encore à savoir s'il n'y a pas un intérêt plus grand à substituer la culture agricole à la culture forestière la plus profitable. En cas de défrichement, le sol transformé en terre arable serait susceptible de produire un revenu de $50 \mathrm{fr}$.; mais comme il est stipulé que l'opération devra s'effectuer en cinq ans et par $5^{e}$ de surface, et que d'ailleurs les terres ne pourront être affermées qu'un an après le défrichement, il s'ensuit que chaque parcelle défrichée fournira un revenu annuel et continu de $50 \times 12=600 \mathrm{fr}$. que l'on commencera à percevoir dans deux ans pour la première parcelle, dans trois ans pour la seconde, etc., dans six ans pour la $5^{e}$. Le capital correspondant s'obtiendra à l'aide du Tarif IV et exprimera ce que vaut actuellement le fonds, dans l'hypothèse où le défrichement s'opérerait suivant les conditions qui viennent d'être énoncées. 
(3) Fonds $=600 \times(24,038+23,114+22,225$

$+21,370+20,548)=66777 \mathrm{fr}$.

Ce résultat comparé au précédent fait voir que le défrichement de la futaie et la transformation du sol en terre arable est commandé par l'intérêt pécuniaire du propriétaire.

II.

L'opportunité du défrichement étant démontrée, il reste à déterminer la valeur réelle de la propriété, d'après les conditions de l'énoncé. Or il a été établi que la valeur brute de la superficie est $616432 \mathrm{fr}$. Mais comme l'acquéreur entend réaliser un bénéfice de $10 \mathrm{p} .0 / 0$ sur la rente des bois exploités, cette valeur se réduit pour lui à :

$616432-61643=534789 \mathrm{f}$.

d'ailleurs le fonds vaut.......... 66777

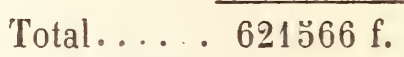

et, en déduisant le capital des frais qui, suivant le Tarif IV, serait de :

$$
60 \times 5 \times 25=7500 \mathrm{fr} .
$$

on obtient pour la valeur nette en fonds et superficie de la propriété :

$621566-7500=614066 \mathrm{fr}$. 
PROBLĖME Ne 6.

VENTE DE FONDS ET SUPERFICIE, AVEC FACULTÉ DE DÉFRICHBR.

On met en vente, avec faculté de défricher, une portion de forêt peuplée d'un taillis simple, et comprenant 60 hectares dont :

10 hect. de bois âgés de 15 ans,

15 hect. - $\quad 18$ ans,
20 hect. $\quad 22 \mathrm{ans}$,
15 hect. $\quad 25$ ans.

La superficie des bois âgés le 15 ans vaut sur pied 400 fr. par $h$.

$\begin{array}{llll}- & 18 \mathrm{ans} & - & 520 \mathrm{fr} . \\ - & 22 \mathrm{ans} & - & 780 \mathrm{fr} \text { - } \\ - & 25 \mathrm{ans} & - & 900 \mathrm{fr} .\end{array}$

Les frais de garde et d'impôt sont de כ̌ fr. par hectare.

On demande quelle est la valeur en fonds et superficie de cette propriété, pour un acquéreur qui voudrait placer ses capitaux à $3 \mathrm{p}$. $0 / 0$ et réaliser sur l'opération du défrichement, ou sur la vente des hois qui en proviendront, un bénéfice de $10 \mathrm{p} .0 / 0$.

On admet :

$1^{\circ}$ Que chaque parcelle pourra être affermée, un an après le défrichement, au prix de $42 \mathrm{fr}$. l'hectare;

$2^{\circ}$ Que l'impôt, à la charge de l'acheteur, sur les terres défrichées, sera de 5 fr. par hectare;

$3^{\circ}$ Que l'acquéreur sera obligé, aussitôt le défrichement commencé, de construire un bâtiment d'exploitation d'une valeur de 6000 fr.;

$4^{\circ}$ Que la vente des bois de souche courrira les frais spéciaux du défrichement. 
SOLUTION.

I.

La propriété étant mise en vente avec faculté de défricher, la première question à résoudre est celle de savoir si l'opération du défrichement sera favorable aux intérêts de l'acheteur. A cet effet, nous allons déterminer quelle est la plus grande valeur que l'on puisse assigner à la propriété :

$1^{\circ}$ Dans le cas où la culture forestière serait conservée;

$2^{\circ}$ Dans le cas où l'on substituerait la culture des céréales à la culture des bois.

On sait ce que l'hectare de bois peut rapporter, suivant qu'on exploite à 15 ans, à 18 ans, à 22 ans ou à 25 ans. Si donc on capitalise, au moyen $\mathrm{d} u$ Tarif III, les différents revenus périodiques de l'hectare correspondant à ces âges, la comparaison de ces résultats entre eux fera connaître en même temps le terme de l'exploitabilité commerciale du matériel ligneux et la plus grande valeur qu'on puisse assigner à la propriété, dans le cas où la culture des bois serait conservée. On trouverait ainsi, que la valeur du fonds, au début de la révolution, serait par hectare de :

$400 \times 1,792=717$ f., sil'exploit. avait lieu à 15 ans, $520 \times 1,424=750 \mathrm{f}, \quad \quad-\quad 18$ ans, $780 \times 1,092=852 \mathrm{f}, \quad \quad \quad-\quad 22$ ans, $900 \times 0,914=823 \mathrm{f} ., \quad-\quad 25 \mathrm{ans}$, 
PROBLÈMES.

D'où l'on peut conclure, que la révolution la plus avantageuse à adopter, dans le cas où la culture en nature de bois serait conservée, est celle de 22 ans. On voit de plus que, si l'on exploitait immédiatement toute la superficie, la plus grande valeur du fonds, considéré comme sol forestier peuplé d'un taillis simple, serait de $\$ 52 \times 60$ moins le capital des frais ou $60 \times 5 \times 33,333$, c'est-à-dire:

$$
51120-10000=41120 \mathrm{fr} \text {. }
$$

Or, on admet que tout ce qui sera défriché pourra être affermé, un an après le défrichement, au prix de $42 \mathrm{fr}$. par hectare ou de $2520 \mathrm{fr}$. pour la totalité. Si donc on procédait de suite à cette opération, la valeur brute du terrain défriché serait, d'après le Tarif IV :

$$
60 \times 42 \times 32,362=81552 \mathrm{fr} . \ldots 81552 \mathrm{f} .
$$

dont il faut retrancher, pour avoir la valeur nette, le capital des frais, savoir :

pour l'impôt $300 \times 33,333=10000 \mathrm{fr}$. pour construction......66 6000$\} \quad 16000 \mathrm{f}$.

Reste........ $65552 \mathrm{f}$.

En comparant ce résultat avec celui qui exprime la valeur du fonds considéré comme sol forestier, on voit que le propriétaire ou l'acquéreur trouvera un très-grand intérêt à substituer la culture agricole à la culture forestière. 
II.

L'opportunité du défrichement étant établie, il importe encore de savoir s'il faut l'opérer immédiatement sur toute la surface du terrain boisé, ou bien s'il ne convient pas de défricher d'abord les deux parcelles âgées de 22 et de 25 ans, et d'attendre que les bois les plus jeunes aient atteint 22 ans avant de les soumettre à la même opération.

Pour se fixer sur ce point, on remarquera que, si on retardait l'exploitation de la première parcelle jusqu'à ce que les bois eussent atteint 22 ans, on ferait un bénéfice de $780-400=380$ fr. par hectare.

Par contre, on perdrait : $1^{\circ}$ l'intérêt pendant 7 ans de la somme qu'on aurait pu réaliser en exploitant à 15 ans; $2^{\circ}$ le prix, avec les intérêts accumulés, de 7 années de fermage du terrain défriché.

La première partie de cette perte, calculée par le Tarif I, donne :

$$
400 \times 1,230-400=92 \text { fr. }
$$

Quant à la seconde partie de la perte, on remarque qu'elle se compose de 7 annuités de 42 fr., ayant crû avec intérêts, la première pendant 7 ans, la seconde pendant 6 ans, etc., la dernière pendant un an. Sa valeur, au bout de 7 ans, serait égale au prix de fermage multiplié par la somme des sept premiers coefficients du Tarif I, soit :

$$
42 \times 6,792=331 \text { fr. } 46 .
$$


Nais cette somme doit être escomptée pour 2 ans, puisque le premier fermage ne pourra être perçu qu'à la fin de la $2^{\mathrm{e}}$ année, et l'on obtient pour la seconde partie de la perte (Tarif II) :

$$
331,46 \times 0,943=313 \mathrm{fr} .
$$

Faisant la somme $92+313=405 \mathrm{fr}$., et comparant cette perte au gain qui résulterait du retard apporté au défrichement, on voit qu'il y a intérêt pour le propriétaire à défricher immédiatement les 10 hectares de bois âgés de 15 ans.

En opérant de la même manière sur les bois âgés de 18 ans, on trouve, qu'en retardant leur exploitation jusqu'à 22 ans, on bénéficierait en 4 ans de $780-520=260 \mathrm{fr}$. par hectare, tandis que l'on perdrait :

$$
\begin{array}{r}
1^{\circ} \ldots \ldots 520 \times 1,125-520=65 \mathrm{fr} . \\
2^{\circ} \ldots \ldots 42 \times 4,309 \times 0,944=171 \\
\text { Total ... } 236 \mathrm{fr} .
\end{array}
$$

En comparant le gain à la perte qui résulterait du retard apporté au défrichement des bois âgés de 18 ans, on voit qu'il y a avantage pour le propriétaire à ne défricher les bois actuellement âgés de 18 ans, que lorsque ces bois auront atteint le terme de leur exploitabilité commerciale, ou 22 ans.

III.

Cela posé, l'estimation de la valeur en fonds et superficie devient facile. 
Superficie. - Les trois parcelles qui renferment les bois âgés de 15 ans, de 22 ans et de 25 ans, devant être défrichées immédiatement, donneront un produit de :

$$
400 \times 10+780 \times 20+900 \times 15=33100 \mathrm{fr} .
$$

Quant à la superficie de la parcelle qui renferme les bois âgés de 18 ans, comme elle ne devra être exploitée que dans 4 ans et qu'alors elle fournira un produit de $780 \mathrm{fr}$. par hectare, sa valeur actuelle, calculée par le Tarif II, sera :

$$
780 \times 15 \times 0,889=10401 \mathrm{fr} .
$$

Par suite, la valeur actuelle totale de la superficie égale $33100+10401=43501 \mathrm{fr}$.

Mais sur cette valeur l'acquéreur veut réaliser un bénéfice de $10 \mathrm{p} .0 / 0$ ou $4350 \mathrm{fr}$. Par conséquent, la valeur qu'il peut offrir de la superficie se réduit à :

(1) . . 43501-4350 = $39151 \mathrm{fr}$.

Fonds. - Les trois parcelles qui doivent être défrichées immédiatement contiennent ensemble 45 hectares qui seront affermés à raison de $42 \mathrm{fr}$. par hectare, un an après le défrichement. Elles fourniront, par conséquent, un revenu annuel et continu de :

$$
45 \times 42=1890 \mathrm{fr} .
$$

que l'on commencera à toucher à l'expiration de la $2^{\circ}$ année. Le Tarif IV donne pour le capital correspondant à ce revenu ;

$$
1890 \times 32,362=61164 \mathrm{fr} .
$$


Quant à la parcelle dont le défrichement n'aura lieu que dans 4 ans, elle fournira un revenu annuel et continu de $15 \times 42=630 \mathrm{fr}$. que l'on commencera à toucher dans 6 ans seulement. Le Tarif IV donne pour le capital équivalent à ce revenu :

$$
630 \times 28,754=18115 \mathrm{fr} .
$$

D'où, en faisant la somme, on trouve pour la valeur actuelle totale du fonds :

$$
\text { (2) . . .61164+18115=79279 fr. }
$$

Et pour la valeur brute de la superficie et du fonds :

$$
\text { (3) . . } 39151+79279=118430 \mathrm{fr} \text {. }
$$

Dont il faut déduire, pour avoir la valeur nette, le capital des frais, savoir :

pour l'impôt.....6 $60 \times 5 \times 33,333=10000 \mathrm{f}$. pour construction............. 6000

$$
\text { Total....... } \overline{16000 \mathrm{f}} \text {. }
$$

Reste (4). . $102430 \mathrm{fr}$.

\section{PROBLÈME No $\%$.}

CANTONNEMENT DE DROITS D'USAGE.

Une sapinière de l'Etat, soumise au traitement du jardinage, est grevée, au profit du propriétaire d'une usine, d'un droit d'usage en bois dont la délivrance annuelle représente une valeur moyenne de $2000 \mathrm{fr}$.

On demande quelle est l'étendue de forêt à abandonner en 
cantonnement à l'usager, dans l'hypothèse où le taux des placements en fonds de terre dans la localité serait de $4 \mathrm{p} .0 / 0$, et le taux de capitalisation de l'émolument usager de 5 p. $0 / 0$.

SOLUTION.

I.

Après avoir déterminé la partie de la forêt dans laquelle le cantonnement devra être assis, on a constaté d'abord que tous les arbres ayant plus de $0^{\mathrm{m}} 50$ de diamètre, à 1 mètre du sol, avaient atteint le terme de leur exploitabilité commerciale, et que la valeur immédiatement réalisable de ces arbres s'élevait, en moyenne, à 180 fr. par hectare.

Celte opération préliminaire terminée, on a procédé à la détermination ảes lois de l'accroissement matériel du peuplement dans son ensemble, et l'on a trouvé, qu'après l'enlèvement des arbres exploitables, le massif restant serait encore susceptible de produire annuellement 4 mètres cubes de bois, par hectare, dont la valeur a été estimée au prix moyen de 10 fr. par mètre cube sur pied.

Enfin on a calculé que les frais annuels de garde, d'impôt, etc., à la charge de l'usager devenu propriétaire, s'élèveraient à 5 fr. par hectare.

II.

L'émolument usager étant équivalent à un revenu annuel de $2000 \mathrm{fr}$, si on capitalise cette somme, 
PROBLÈMES.

suivant les conditions de l'énoncé, c'est-à-dire au denier 20 , le résultat exprimera la valeur nette que devra représenter actuellement la partie de forêt à abandonner en toute propriété à l'usager, soit $40000 \mathrm{fr}$.

Or, on a vu qu'un hectare pouvait fournir un revenu annuel et constant de 4 mètres cubes à $10 \mathrm{fr}$. l'un, ou de $40 \mathrm{fr}$. Si donc on capitalise ce revenu à 4 p. $0 / 0$, taux ordinaire des placements en fonds de terre dans la localité, on obtient pour la valeur en fonds et superficie de l'hectare :

$$
\text { (TarifIV) } \ldots 40 \times 25=1000 \mathrm{fr} \text {. }
$$

Mais à cette valeur il faut encore ajouter celle des arbres immédiatement exploitables, qui a été estimée à $180 \mathrm{fr}$. par hectare; ce qui porte à $1180 \mathrm{fr}$. la valeur actuelle en fonds et superficie de l'hectare.

D'où l'on déduit, pour le nombre d'hectares à abandonner à l'usager :

$$
n=\frac{40000}{1180}=33 \text { hect. } 89 \text { ares } 83 .
$$

III.

Telle serait l'étendue de forêt que l'on devrait abandonner à l'usager, à titre de cantonnement de son droit, dans le cas où l'on ne devrait pas tenir compte des frais de garde et d'impôt qu'il aura à supporter, lorsqu'il sera devenu propriétaire.

Mais si, par le cantonnement même, on devait 
DE L'ESTHATION EN FONDS ET SUPERFICIE.

l'indemniser de ces frais, on voit de suite qu'il suffirait de les porter en déduction du produit annuel de l'hectare. Dès lors la valeur nette en fonds et superficie de l'hectare serait :

$$
35 \times 25+180=1055 \mathrm{fr} .
$$

et l'étendue à abandonner à l'usager :

$$
n=\frac{40000}{105 \%}=57 \text { hect. } 91 \text { ares } 47 .
$$

PROBLÈVE NO 8.

CANTONNEMENT DE DROITS D'USAge.

Une forêt appartenant à un particulier est grevée, au profit d'une commune, d'un droit d'usage en bois de marnage et de chauffage dont la délivrance annuelle est estimée à une valeur de $9000 \mathrm{fr}$. La forêt usagère est exploitée en taillis composé, à une révolution de 2 ans. Le taillis est peuplé en grande majorité de charmes et de hêtres mêlés de quelques chênes et de bois blancs; la réserve se compose pour la plus grande partie de chênes et de quelques hêtres et charmes.

On demande quelle est l'étendue de forêt à abandonner à la commune usagère pour le rachat de ses droits, en admettant :

$1^{\circ}$ Que le taux des placements en fonds de terre dans la localité soit de 3 p. $0 / 0$;

$2^{\circ}$ Que les frais de garde et d'impôt s'élèvent annuellement à fr. par hectare. 
SOLUTTON.

I.

La valeur des délivrances annuelles étant de $9000 \mathrm{fr}$., si on capitalise cette somme au denier 20, c'est-à-dire, au taux de $5 \mathrm{p} .0 / 0$, le résultat :

$$
.9000 \times 20=180000 \text { fr. }
$$

exprimera la valeur à assigner au cantonnement, autrement dit, la valeur que doit représenter la partie de forêt qui sera abandonnée à la commune usagère, au moment où elle en prendra possession.

Le problème à résoudre consiste donc à détacher de la forêt grevée une portion telle que, si on voulait la vendre immédiatement, on pût en tirer un prix égal à $180000 \mathrm{fr}$. Pour procéder à cette estimation, il est nécessaire d'abord de déterminer la partie ou les parties de la forêt dans lesquelles le cantonnement devra être assis, puis de séparer sur le terrain les peuplements qui diffèrent entre eux par l'âge des bois, la consistance des massifs, la fertilité des terrains, et de mesurer l'étendue respective de ces parcelles. Cela fait, on procède séparément à l'estimation de chaque parcelle, de la manière que nous allons indiquer en raisonnant sur un cas particulier bien défini.

II.

Supposons que l'une des parcelles à comprendre dans le cantonnement, la parcelle $A$, renferme 
12 hect. 90, lesquels ont été récemment exploités en trois coupes d'égale contenance et sont peuplés d'un recru âgé de 1 à 3 ans.

Les renseignements recueillis sur l'exploitation de ces trois coupes, dont tous les bois ont été délivrés à la commune usagère, font connaître qu'elles ont fourni en produits effectifs :

$1^{\circ} 24534$ fagots d'une valeur de $20 \mathrm{fr}$. le cent, façon déduite....... 4906 fr. 80

$2^{\circ} 1386$ stères de bois de feu à 5 fr. l'un, tous frais déduits....... 6930 "

(Ces produits ont été délivrés en affouage aux habitants.)

$3^{\circ} \mathrm{Un}$ nombre indéterminé de pièces de chêne, provenant de la réserve et dont la vente a produit..... $10743 \quad 15$

Total..... $\overline{22579 \text { fr. } 95}$

Soit par hectare $1750 \mathrm{fr}$.

Mais au moment de leur exploitation, ces trois coupes étaient âgées, la première de 26 ans, la deuxième de 27 ans, la troisième de 28 ans, ou en moyenne de 27 ans, de sorte que, pour avoir leur production probable à l'âge ordinaire d'exploitation, ou à 25 ans, il faut retrancher du produit qu'elles ont fourni, deux années d'accroissement soit $130 \mathrm{fr}$., et l'on a pour la valeur de l'hectare exploitable à 25 ans... $1750-130=1620$ fr. qui peuvent se décomposer ainsi qu'il suit : 
produit du sous-bois à 25 ans........ $420 \mathrm{fr}$. produit de la futaie de chêne (bois d'œuvre) 770 produit de la futaie blanche (hêtre et charme) et des branches de la futaie de chêne. 430

Si l'on voulait déduire de ces données la valeur en fonds et superficie de la parcelle à estimer, rien ne serait plus facile. Mais on pourrait objecter, et avec raison, que ces chiffres ne prouvent pas que le traitement antérieurement suivi soit le plus favorable à la production, ni que le peuplement, ainsi qu'il est constitué, soit susceptible de fournir le même revenu dans l'avenir. D'où il résulterait que, si on voulait calculer la valeur capitale de la parcelle en fonction d'un revenu présumé de $1620 \mathrm{fr}$. par hectare à 25 ans, on s'exposerait à commettre une erreur, soit en moins, soit en plus. Cette objection conduit forcément l'estimateur à résoudre d'abord la double question du choix de la révolution et du régime à appliquer.

III.

A cet effet, il est nécessaire d'estimer d'abord quelle serait la production probable du taillis aux différents âges qui pourraient être pris pour terme de la révolution. Or, en examinant la composition du sous-bois, on remarque qu'il est peuplé en grande majorité de charme et de hêtre, deux essences dont la première croît très-lentement en taillis et ne 
donnerait avant l'âge de 20 ans que du menu bois de très-peu de valeur dans la contrée, et dont l'autre ne peut être soumise à une révolution de plus de 30 ans, sans que la production des souches soit compromise; 20 et 30 ans sont donc des limites entre lesquelles la révolution du taillis doit être comprise. Si on compare le peuplement de la parcelle à estimer avec d'autres peuplements placés dans les mêmes conditions de végétation, et si l'on trouve que le taillis sur lequel on opère peut fournir :
à 20 ans, un produit net de... 300 fr.
à 25 ans, un produit net ảe.. 420
à 30 ans, un produit net de... 550

on en concluera qu'il n'y a pas d'intérêt à changer la révolution de 25 ans qui a été appliquée jusqu'àlors au taillis; car, si on capitalise ces trois produits, à 3 p. $0 / 0$, comme des revenus à toucher périodiquement, le premier tous les 20 ans, le second tous les 25 ans, le troisième tous les 30 ans, on obtient, par le Tarif III :

pour le $1^{\text {er }}$ capital, $300 \times 1,240=372 \mathrm{fr}$. pour le $2^{\circ}$ id. $420 \times 0,914=384 \mathrm{fr}$. pour le $3^{\mathrm{e}}$ id. $550 \times 0,701=385 \mathrm{fr}$.

Si d'ailleurs on constate que la durée de cette révolution est assez longue pour assurer la bonne venue des arbres qu'on voudrait élever comme réserves sur le taillis, on concluera également que la révolu- 
tion de 25 ans devra être conservée, dans le cas où on voudrait continuer à appliquer le régime du taillis composé.

IV.

Pour se fixer sur ce dernier point, on détermine, d'une part le revenu prohable que l'on pourrait attendre du taillis, s'il était débarrassé du couvert des réserves et ramené à l'état de taillis simple, d'autre part la valeur, au début de la révolution, de tous les arbres réservés au moment de l'exploitation.

Or, en admettant que le revenu net de l'hectare, exploité en taillis simple à la révolution de 25 ans, soit de $550 \mathrm{fr}$., et que la valeur de tous les arbres réservés fût de $776 \mathrm{fr}$. par hectare, au moment de la dernière exploitation, il est évident que, par la conversion du taillis composé en taillis simple, l'hectare vaudra, au début de la révolution :

Valeur de la superficie........... $776 \mathrm{fr}$. Valeur du fonds (Tarif III) $550 \times 0,914=503$

$$
\text { Total.... } \overline{1279 \mathrm{fr}} \text {. }
$$

Reste maintenant à calculer la valeur en fonds et superficie de l'hectare exploitable à 25 ans, en taillis composé. Or, il a été précédemment établi que, dans ces conditions, le sous-bois seul contribuerait au rendement de la coupe à 25 ans pour une somme de $420 \mathrm{fr}$.; il ne s'agit donc plus que d'évaluer le produit probable de la futaie. Dans ce but, on a procédé au comptage, au mesurage et à l'estimation maté- 
rielle de tous les arbres réservés dans la parcelle à estimer. Les résultats de cette opération sont consignés dans le tableau ci-après :

DÉNOMBREMENT DES ARBRES RÉSERVÉS DANS LA PARGELLE A.

\begin{tabular}{|c|c|c|c|c|c|}
\hline ESSENCES. & $\begin{array}{c}\text { CIRCON- } \\
\text { FÉRENCE } \\
\text { à } \\
1 \mathrm{~m}, 30 \\
\text { du sol. }\end{array}$ & $\begin{array}{l}\text { NOMBre } \\
\text { d'ar- } \\
\text { bres. }\end{array}$ & $\begin{array}{c}\text { PRODUIT } \\
\text { par } \\
\text { arbre. }\end{array}$ & $\begin{array}{c}\text { Produit } \\
\text { total } \\
\text { par } \\
\text { catégorie. }\end{array}$ & OBSERVATIONS \\
\hline \multirow{11}{*}{ CHÊNES. } & 0,30 à 0,50 & 369 & $\begin{array}{r}\text { M.c. } \\
0,0535\end{array}$ & $\begin{array}{c}\text { M.c. } \\
19,557\end{array}$ & \multirow{22}{*}{$\begin{array}{l}\text { Les nombres de } \\
\text { la dernière colonne } \\
\text { expriment le cube } \\
\text { de la tige seule- } \\
\text { ment, au quart sans } \\
\text { déduction. } \\
\text { A ce volume, il } \\
\text { faut par conséquent } \\
\text { ajouter celui des } \\
\text { branches qui for- } \\
\text { ment le houppier. } \\
\text { Ce dernier volume, } \\
\text { exprimé en stères } \\
\text { de chauffage, s'ob- } \\
\text { tient en multipliant } \\
\text { le volume des tiges } \\
\text { par le coeficient } \\
1,70, \text { lequel a été } \\
\text { déterminé par des } \\
\text { expériences. } \\
\text { On obtient ainsi } \\
\text { pour le volume des } \\
\text { branches: } \\
342,247 \times 1,70 \\
=581 \text { st. } 820 \text {. }\end{array}$} \\
\hline & 0,66 & 100 & 0,157 & 15,700 & \\
\hline & 0,83 & 140 & 0,227 & $5 !, 780$ & \\
\hline & 1 & 82 & 0,563 & 29,766 & \\
\hline & 1,16 & 59 & 0,541 & 31,919 & \\
\hline & 1,53 & 46 & 0,763 & 55,190 & \\
\hline & 1,50 & 25 & 0,926 & 23,150 & \\
\hline & 1,66 & 14 & 1,252 & 17,248 & \\
\hline & 1,83 & 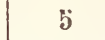 & 1,597 & 7,985 & \\
\hline & $2 n$ & 9 & 2,023 & 18,225 & \\
\hline & 0,66 & 14 & 0,155 & 1,890 & \\
\hline \multirow{5}{*}{ HÊTRES. } & 0,83 & 45 & 0,211 & 9,495 & \\
\hline & $1 "$ & 16 & 0,339 & 5,424 & \\
\hline & 1,16 & 1 & 0,490 & 0,490 & \\
\hline & 1,35 & 8 & 0,673 & 5,400 & \\
\hline & 1,50 & 3 & 0,871 & 2,615 & \\
\hline \multirow{6}{*}{\begin{tabular}{|} 
Charmes \\
et \\
divers.
\end{tabular}} & 0,30 à 0,50 & 1180 & 0,045 & 55,100 & \\
\hline & 0,66 & 167 & 0,155 & 22,545 & \\
\hline & 0,83 & 39 & 0,211 & 8,229 & \\
\hline & 1 . & 6 & 0,539 & 2,034 & \\
\hline & 1,16 & 1 & 0,507 & 0,507 & \\
\hline & & & & 342,247 & \\
\hline
\end{tabular}


Telle est, dans son ensemble, la composition de la réserve existant dans les trois coupes de la parcelle A.

Ce dénombrement opéré, il a fallu procéder à la classification des réserves par catégories, c'est-à-dire, en baliveaux de l'âge, modernes, anciens de 3 âges, etc. A cet effet, des expériences d'accroissement ont été faites sur un certain nombre de chênes en grume qui gisaient encore sur le parterre de la coupe exploitée la dernière, et sur quelques hêtres et charmes que l'on a fait abattre dans un peuplement voisin de cette coupe. Ces expériences ont établi que :

Tous les chênes qui mesurent $0^{\mathrm{m}}, 66$ à $0^{\mathrm{m}}, 83 \mathrm{de}$ tour à $1^{\mathrm{m}}, 30 \mathrm{du}$ sol, sont des baliveaux de deux âges, c'est-à-dire, des modernes de 50 ans;

Tous ceux dont la circonférence est comprise entre $1^{\mathrm{m}}, 00,1^{\mathrm{m}}, 16,1^{\mathrm{m}}, 33$ sont des anciens de trois âges ou de 75 ans;

Tous ceux qui mesurent $1^{\mathrm{m}}, 50,1^{\mathrm{m}}, 66,1^{\mathrm{m}}, 83 \mathrm{de}$ tour sont des anciens de quatre âges ou de 100 ans;

Tous ceux qui atteignent $2^{\mathrm{m}}, 00,2^{\mathrm{m}}, 16,2^{\mathrm{m}}, 33$ sont des arbres de 125 ans.

Or, en appliquant à chaque unité de volume les prix les plus modérés du commerce, dans la localité, savoir :

0 fr. 60 cent. pour un baliveau de l'àge, quelle qu'en soit l'essence;

$2 \mathrm{fr}$. le décistère (au quart sans déduction) pour les chênes de $0^{\mathrm{m}}, 66$ à $0^{\mathrm{m}}, 83$ de tour; 
$3 \mathrm{fr}$. le décistère pour les pièces de $1^{\mathrm{m}}, 00$ et $1^{\mathrm{m}}, 16$ de tour;

$4 \mathrm{fr}$.

id.

de $1^{\mathrm{m}}, 33$;

4 fr. 50

id.

de $1^{\mathrm{m}}, 50$ et au-dessus ;

6 fr. le stère de bois de feu, essence hêtre;

2 fr. le décistère, au quart sans déduction, pour le bois d'industrie, essence hêtre ;

$5 \mathrm{fr}$. le stère debois de feu, rondin de charme; $7 \mathrm{fr} .50$ id. quartier de charme; 3 fr. 33 id. provenant des branches, quelle que soit l'essence ;

On est conduit aux résultats qui sont consignés dans le tableau suivant : 
PROBLÈNES.

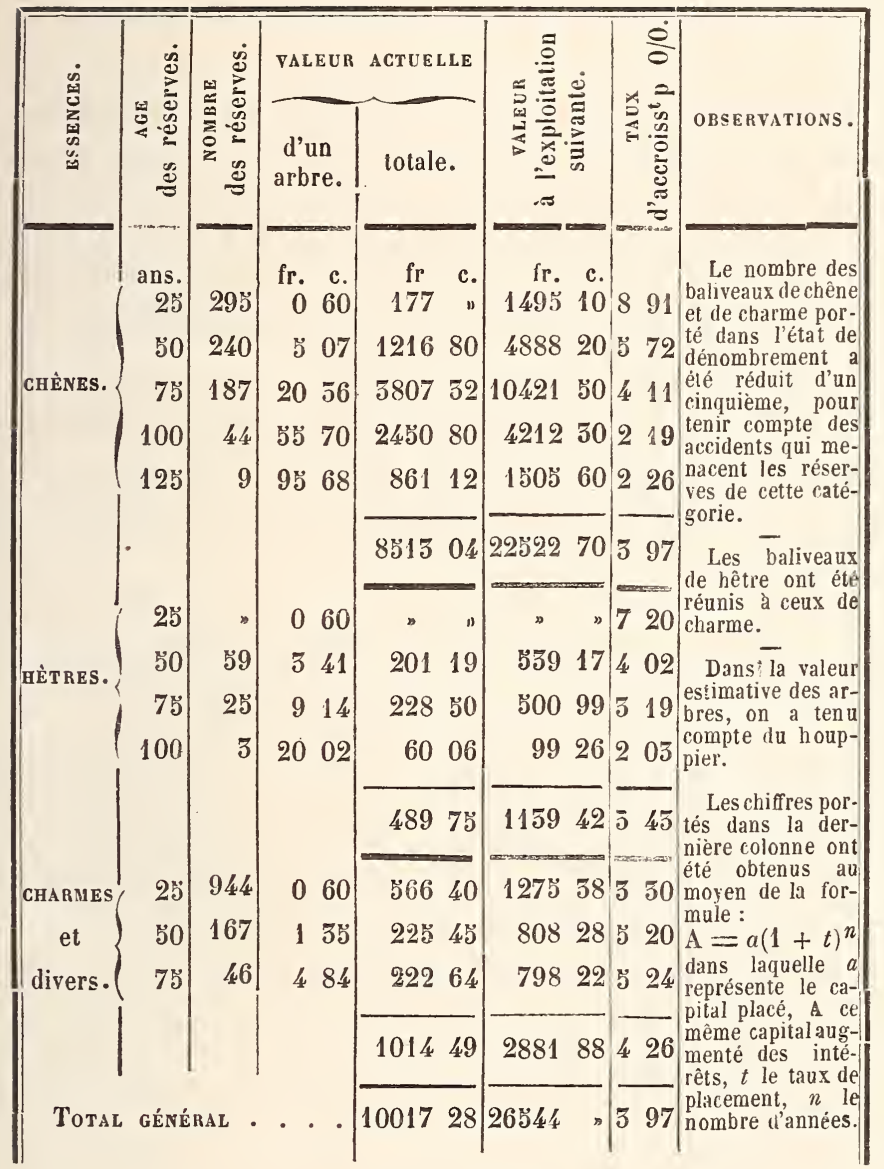

Des chiffres inscrits dans le tableau qui précède, on conclut :

$1^{\circ}$ Que le régime du taillis composé est plus favorable à l'intérêt pécuniaire du propriétaire que le régime du taillis simple, puisque la valeur de tous les arbres qu'on réserve à chaque exploitation du 
taillis s'accroît, d'une exploitation à la suivante, comme un capital placé à 3,97 p. 0/0, taux supérieur à celui des placements en biens-fonds dans la localité. Qu'en conséquence, il y a lieu de procéder à l'estimation de la valeur en fonds et superficie de la parcelle A, en prenant pour base de cette opération la condition que le régime du taillis composé sera conservé.

$2^{\circ}$ Que si, dans l'avenir, on constitue la réserve sur les bases du balivage actuel, la futaie devra produire, à chaque exploitation du taillis, une somme égale à :

$$
\begin{gathered}
26344 \text { fr. } 00-10017 \mathrm{fr} .28=16326 \mathrm{fr} .72 . \\
\text { soit. . } 1281 \mathrm{fr} \text {. par hectare. }
\end{gathered}
$$

Ajoutant cette somme au produit présumé du sous-bois qui a été porté à $420 \mathrm{fr}$. par hectare, on obtient pour le rendement probable, par hectare, à chaque exploitation, un total de $1701 \mathrm{fr}$.

Si maintenant on cherche, au moyen du Tarif III, la valeur, au début de la révolution, d'un revenu de $1701 \mathrm{fr}$. à toucher périodiquement tous les 25 ans, on trouve:

$$
1701 \times 0,914=1555 \mathrm{fr} .
$$

tandis que si on réalisait le capital engagé dans la réserve et que l'on transformât le taillis composé en taillis simple, la valeur de l'hectare, au début de la révolution, ne serait que de $1279 \mathrm{fr}$.

Les chiffres du dernier tableau font voir en outre 
que si on réduisait le balivage aux seuls arbres compris dans les catégories de baliveaux de l'âge, modernes et anciens de trois âges pour les chênes, baliveaux de l'âge, modernes et anciens de trois âges pour les hêtres, baliveaux de l'âge, modernes et anciens de trois âges pour les charmes, le capital engagé dans ces réserves se réduirait :

Au début de la révolution, à....... 664 b̆ f.

Que ce capital atteindrait, à l'exploitation suivante, une valeur de........ $20627 \mathrm{f}$. et fonctionnerait, par conséquent, comme une somme placée à $4,54 \mathrm{p} .0 / 0$.

Dans cette hypothèse, on serait amené à réaliser, au début de la révolution, la valeur des chênes anciens de quatre et de cinq âges et des hêtres anciens de quatre âges, soit, au total, une somme de $3372 \mathrm{f}$. et par hectare............... $261 \mathrm{f}$.

De plus on pourrait bien admettre que, par suite de la diminution du couvert, le revenu du taillis s'élevât de 420 à................ $430 \mathrm{f}$.

D'ailleurs le produit total de la réserve dans le rendement de chaque coupe serait de 20627 - 6645 $=13982 \mathrm{fr}$., soit, par hectare....... $1084 \mathrm{f}$.

D'où il résulterait que la valeur en fonds et superficie de l'hectare, au début de la révolution, serait représentée par :

la valeur des réserves immédiatement exploita- 


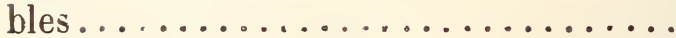

le capital correspondant à un revenu périodique de $430 \times 1084=1514 \mathrm{fr}$. ̀̀ toucher pour la première fois dans 25 ans ; soit (Tarif III) $1514 \times 0,914=\ldots \ldots \ldots \quad 1383 \mathrm{f}$.

Total....... $1644 \mathrm{f}$.

En comparant ce résultat avec celui que l'on obtiendrait, dans le cas où l'on conserverait le balivage existant, on trouve une différence en plus, de 1644 - $1555=89 \mathrm{fr}$. pour la valeur de l'hectare, au début de la révolution.

Néanmoins, il peut y avoir intérêt pour le propriétaire à conserver le balivage existant, afin de pouvoir comprendre dans chaque coupe un certain nombre de gros chênes, dont la valeur exceptionnelle facilite toujours la vente, à un prix plus avantageux, des pièces de faible dimension, telles que les petits modernes. Dans ce cas, la valeur de l'hectare, au début de la révolution, serait définitivement fixée à................... 15 วั $\mathrm{f}$. et le revenu probable, à l'âge fixé pour l'exploitation, à................ $1701 \mathrm{f}$.

En prenant ces chiffres pour base de l'estimation, la valeur en fonds et superficie de la parcelle A est facile à déterminer. En effet, on se souvient que cette parcelle comprend trois coupes contenant chacune 
4 h. 30 ares, et peuplées, la première, d'un recru de 3 ans, la seconde d'un recru de deux ans et la dernière d'un recru d'un an. La première sera exploitée dans 22 ans et fournira un produit de 1701 fr. La valeur actuelle de la superficie de cette coupe s'obtiendra à l'aide du Tarif II et sera :

$$
4,30 \times 1701 \times 0,522=3818 \mathrm{fr} .
$$

La valeur du fonds, calculée par les Tarifs II et III, sera : $4,30 \times 1555 \times 0,522=3490$ fr. et en faisant la somme, on aurait pour la valeur en fonds et superficie de la première coupe :

$$
4,30 \times(1701+1555) \times 0,522=7308 \text { fr. }
$$

La valeur en fonds et superficie de la seconde coupe s'obtiendrait de la même manière et serait :

$$
4,30 \times 3256 \times 0,507=7098 \mathrm{fr} .
$$

Enfin la valeur actuelle en fonds et superficie de la dernière coupe serait :

$$
4,30 \times 3256 \times 0,492=6888 \mathrm{fr} .
$$

D'où, en faisant la somme des trois derniers résultats, on obtient pour la valeur actuelle en fonds et superficie de la parcelle $\mathrm{A}$ :

$$
7308+7098+6888=21294 \mathrm{fr} .
$$

Mais de cette somme il faut encore déduire le capital des frais, lesquels s'élèvent annuellement à $5 \mathrm{fr}$. par hectare, et par conséquent à $64 \mathrm{fr}$. 50 pour 12 hect. 90. Ce capital s'obtient à l'aide du Tarif IV et serait égal à : 


$$
64,50 \times 33,333=2150 \mathrm{fr} .
$$

La valeur actuelle nette de la parcelle à estimer serait donc de :

$$
21294-2150=19144 \mathrm{fr} .
$$

VI.

On procéderait, d'une manière analogue, à l'estimation séparée des parcelles $\mathrm{B}, \mathrm{C}, \mathrm{D}$, etc. à comprendre dans le cantonnement, jusqu'à ce que l'on eût déterminé sur le terrain une étendue de peuplements dont la valeur actuelle totale serait égale à la valeur du droit capitalisé.

Noта. Nous ferons observer que, dans l'estimation de la parcelle $A$, nous avons tenu compte des frais de garde et d'impôt, afin d'obtenir la valeur nette actuelle de cette parcelle. En matière de cantonnement, la question de savoir si on doit indemniser l'usager des frais qu'il aura à supporter, comme propriétaire, doit être décidée d'avance par les tribunaux ou par les parties. Quant à nous, nous pensons que dans les cantonnements qui intéressent les particuliers, soit comme propriétaires, soit comme usagers, les frais de garde, d'impôt, etc., devraient être laissés à la charge de l'usager, en compensation de l'avantage qu'il trouve à passer de l'état d'usager à la qualité de propriétaire. 


\section{TARIE I. \\ $x=(1+t)^{n}$}

Ce tarif donue les facteurs par lesquels il fat multiplier les sommes placées à intérêts composés, pour avoir leur valeur, en principal et intérêts accumulés, ì l'expiration d'un nombre d'années déterminé.

Les nombres portés dans ces tables sont extraits des tarifs de Colla jubliés par M de Salomon; on a seulement complété la série, d'année en annéc, jusqu'a 150 ans, d'après les formules. 
TARIF I.

\begin{tabular}{|c|c|c|c|c|c|}
\hline \multirow{2}{*}{ 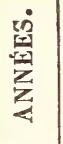 } & \multicolumn{5}{|c|}{ TAUX D'INTÉRÊT. } \\
\hline & 3 p. $0 / 0$. & $3 \frac{1}{2}$ p. $0 / 0$ & 4 p. $0 / 0$ & $4 \frac{1}{2}$ p. $0 / 0$ & ऽ p. $0 / 0$. \\
\hline 1 & 1,030 & 1,035 & 1,040 & 1,045 & 1,0 วั0 \\
\hline 2 & 1,061 & 1,071 & 1,082 & 1,092 & 1,102 \\
\hline 3 & 1,093 & 1,109 & 1,125 & 1,141 & 1,158 \\
\hline 4 & 1,125 & 1,148 & 1,170 & 1,193 & $1,210 ّ$ \\
\hline 5 & $:, 159$ & 1,188 & 1,217 & 1,246 & 1,276 \\
\hline 6 & 1,194 & 1,229 & 1,265 & 1,302 & 1,340 \\
\hline 7 & 1,230 & 1,272 & 1,316 & 1,361 & 1,407 \\
\hline 8 & 1,267 & 1,317 & 1,369 & 1,422 & 1,477 \\
\hline 9 & 1,305 & 1,363 & 1,423 & 1,486 & 1,551 \\
\hline 10 & 1,344 & 1,411 & 1,480 & 1,553 & 1,629 \\
\hline 11 & 1,384 & 1,460 & 1,539 & 1,623 & 1,710 \\
\hline 12 & 1,425 & 1,511 & 1,601 & 1,696 & 1,796 \\
\hline 13 & 1,469 & 1,564 & 1,665 & 1,772 & 1,886 \\
\hline 14 & 1,513 & 1,619 & 1,732 & 1,852 & 1,980 \\
\hline 15 & 1,558 & 1,675 & 1,801 & 1,935 & 2,079 \\
\hline 16 & 1,605 & 1,734 & 1,873 & 2,022 & 2,183 \\
\hline 17 & 1,653 & 1,793 & 1,948 & 2,113 & 2,292 \\
\hline 18 & 1,702 & 1,857 & 2,026 & 2,208 & 2,407 \\
\hline 19 & 1,753 & 1,922 & 2,107 & 2,308 & 2,527 \\
\hline 20 & 1,806 & 1,990 & 2,191 & 2,412 & 2,653 \\
\hline 21 & 1,860 & 2,059 & 2,279 & 2,520 & 2,786 \\
\hline
\end{tabular}


EN FONDS ET SUPERPICIE.

TATIF I.

\begin{tabular}{|c|c|c|c|c|c|}
\hline \multirow{2}{*}{ 窗 } & \multicolumn{5}{|c|}{ TAUX D'INTÉRÊT. } \\
\hline & 3 p. $0 / 0$. & $3 \frac{1}{2}$ p. $0 / 0$ & 4 p. $0 / 0$ & $4 \frac{1}{2}$ p. $0 / 0$. & $5 \mathrm{p} .0 / 0$. \\
\hline 22 & 1,916 & 2,132 & 2,370 & 2,634 & 2,925 \\
\hline 23 & 1,974 & 2,206 & 2,465 & 2,752 & 3,072 \\
\hline 24 & 2,033 & 2,283 & 2,563 & 2,876 & $3,22 כ$ \\
\hline $20 ّ$ & 2,094 & 2,363 & 2,666 & 3,005 & 3,386 \\
\hline 26 & 2,157 & 2,446 & 2,772 & 3,141 & 3,556 \\
\hline 27 & 2,221 & 2,332 & 2,883 & 3,282 & 3,733 \\
\hline 28 & 2,288 & 2,620 & 2,999 & 3,430 & 3,920 \\
\hline 29 & 2,357 & 2,712 & 3,119 & 3,504 & 4,116 \\
\hline 30 & 2,427 & 2,807 & 3,233 & 3,745 & 4,322 \\
\hline 31 & 2,500 & 2,905 & 3,373 & 3,914 & 4,538 \\
\hline 32 & 2,575 & 3,007 & 3,508 & 4,090 & 4,765 \\
\hline 33 & 2,652 & 3,112 & 3,648 & 4,274 & 5,003 \\
\hline 34 & 2,732 & 3,221 & 3,794 & 4,466 & 5,253 \\
\hline 35 & 2,814 & 3,334 & 3,946 & 4,667 & 5,516 \\
\hline 36 & 2,898 & 3,450 & 4,104 & 4,877 & 5,792 \\
\hline 37 & 2,985 & 3,571 & 4,268 & 5,097 & 6,081 \\
\hline 38 & $3,07 \mathrm{\jmath}$ & 3,696 & 4,439 & 5,326 & 6,385 \\
\hline 39 & 3,167 & 3,825 & 4,616 & 5,566 & 6,705 \\
\hline 40 & 3,262 & 3,959 & 4,801 & 5,816 & 7,040 \\
\hline 41 & 3,360 & 4,098 & 4,993 & 6,078 & 7,392 \\
\hline 42 & 3,461 & 4,241 & 5,193 & 6,352 & 7,762 \\
\hline
\end{tabular}


TARIF I.

\begin{tabular}{|c|c|c|c|c|c|}
\hline \multirow{2}{*}{ 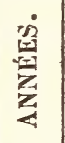 } & \multicolumn{5}{|c|}{ TAUX D'INTÉRÊT. } \\
\hline & 3 p. $0 / 0$ & $3 \frac{1}{2} \mathrm{p} \cdot 0 / 0$ & 4 p. $0 / 0$. & $4 \frac{1}{2}$ p. $0 / 0$ & ร p. $0 / 0$. \\
\hline 43 & 3, วั6ว & 4,390 & $\breve{5}, 400$ & 6,637 & 8,1 วั0 \\
\hline 44 & 3,671 & $4,3,13$ & 5,617 & 6,936 & $8,5 ั ว ั 7$ \\
\hline 45 & 3,782 & 4,702 & 5,841 & 7,248 & $8,98 ว$ \\
\hline 46 & 3,89כ & 4,867 & 6,07 כั & 7,374 & 9,434 \\
\hline 47 & 4,012 & $\check{5}, 037$ & 6,318 & 7,91 วั & 9,906 \\
\hline 48 & 4,132 & 5,214 & 6,571 & 8,271 & 10,401 \\
\hline 49 & 4,2506 & 丂,396 & 6,833 & 8,644 & 10,921 \\
\hline 50 & 4,384 & ร,วั8ว̆ & 7,107 & 9,033 & 11,467 \\
\hline 51 & 4,515 & 5,780 & 7,391 & 9,439 & $12,0 \leq 1$ \\
\hline รั2 & 4,63 & 5,983 & 7,687 & 9,864 & $12,6.43$ \\
\hline 53 & 4,790 & 6,192 & 7,994 & 10,308 & 13,275 \\
\hline$\breve{5}$ & 4,934 & 6,409 & 8,314 & 10,772 & 13,939 \\
\hline 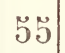 & $5,0 \$ 2$ & 6,633 & 8,646 & 11,230 & 14,636 \\
\hline 56 & $\breve{5}, 23 \breve{~}$ & $6,86 \breve{3}$ & 8,992 & 11,763 & 15,367 \\
\hline 57 & $\check{5}, 392$ & 7,100 & 9,3 ว้2 & 12,292 & 16,136 \\
\hline 58 & ร,ร53 & $7,3 כ ้ 4$ & 9,726 & 12,845 & 16,943 \\
\hline 59 & 5,720 & 7,612 & 10,115 & 13,423 & 17,790 \\
\hline 60 & 5,892 & 7,878 & 10,520 & 14,027 & 18,679 \\
\hline 61 & 6,068 & $8,15 \%$ & 10,947 & 14,639 & 19,613 \\
\hline 62 & 6,250 & 8,439 & 11,378 & 15,318 & $20,0.94$ \\
\hline 63 & 6,438 & $8.73 \mathrm{3}$ & 11,833 & 16,008 & 21,623 \\
\hline
\end{tabular}


EN FONDS ET SUPERFICIE.

TARIF I.

\begin{tabular}{|c|c|c|c|c|c|}
\hline$\dot{\Omega}$ & \multicolumn{5}{|c|}{ TAUX D'INTÉRÊT. } \\
\hline$z_{2}$ & 3 p. $0 / 0$ & $3 \frac{1}{2}$ p. $0 / 0$. & 4 p. $0 / 0$ & $4 \frac{1}{2}$ p. $0 / 0$ & 5 p. $0 / 0$. \\
\hline 64 & 6,631 & 9,040 & 12,306 & 16,728 & 22,705 \\
\hline 65 & 6,830 & 9,357 & 12,799 & 17,481 & 23,840 \\
\hline 66 & 7,035 & 9,684 & 13,311 & 18,267 & 25,032 \\
\hline 67 & 7,246 & 10,023 & 13,843 & 19,089 & 26,283 \\
\hline 68 & 7,463 & 10,374 & 14,397 & 19,948 & 27,0098 \\
\hline 69 & 7,687 & 10,737 & 14,973 & 20,846 & 28,978 \\
\hline 70 & 7,918 & 11,113 & $15, \breve{5} 72$ & 21,784 & 30,426 \\
\hline 71 & 8,155 & 11,502 & 16,194 & 22,764 & 31,948 \\
\hline 72 & 8,400 & 11,904 & 16,842 & 23,789 & 33,545 \\
\hline 73 & 8,652 & 12,321 & 17,516 & 24,859 & 35,222 \\
\hline 74 & 8,912 & 12,752 & 18,217 & 25,978 & 36,984 \\
\hline 75 & 9,179 & 13,199 & 18,945 & 27,147 & 38,833 \\
\hline 76 & 9,454 & 13,660 & 19,703 & 28,369 & 40,774 \\
\hline 77 & 9,738 & 14,139 & 20,491 & 29,645 & 42,813 \\
\hline 78 & 10,030 & 14,633 & 21,311 & 30,979 & 44,954 \\
\hline 79 & 10,331 & 15,146 & 22,163 & 32,373 & 47,201 \\
\hline 80 & 10,641 & $1 ว, 676$ & 23,0500 & 33,830 & 49,561 \\
\hline 81 & 10,960 & 16,224 & 23,972 & 35,353 & 52,040 \\
\hline 82 & 11,289 & 16,792 & 24,931 & 36,944 & 54,641 \\
\hline 83 & 11,628 & 17,380 & $2 \breve{,}, 928$ & 38,606 & 57,374 \\
\hline 84 & 11,976 & 17,988 & 26,965 & 40,343 & 60,242 \\
\hline
\end{tabular}


TARIF I.

\begin{tabular}{|c|c|c|c|c|c|}
\hline \multirow{2}{*}{ 窗 } & \multicolumn{5}{|c|}{ TAUX D'INTÉRÊT. } \\
\hline & 3 p. $0 / 0$ & $3 \frac{1}{2} \mathrm{p} .0 / 0$ & 4 p. $0 / 0$ & $4 \frac{1}{2}$ p. $0 / 0$ & ร p. $0 / 0$. \\
\hline 85 & 12,336 & 18,618 & $28,0.14$ & 42,159 & 63,234 \\
\hline 86 & 12,706 & 19,269 & 29,165 & 44,056 & 66,417 \\
\hline 87 & 13,087 & 19,944 & 30,332 & 46,038 & 69,738 \\
\hline 88 & 13,480 & 20,642 & 31,545 & 48,110 & 73,225 \\
\hline 89 & 13,884 & 21,364 & 32,807 & $50,27 \check{~}$ & 76,886 \\
\hline 90 & 14,300 & ) 22,112 & 34,119 & 52,537 & 80,730 \\
\hline 91 & 14,729 & 22,886 & 35,484 & 54,901 & 84,767 \\
\hline 92 & 15,171 & 23,687 & 36,903 & 57,372 & 89,005 \\
\hline 93 & 15,626 & 24,516 & 38,380 & 59,954 & 93,455 \\
\hline 94 & 16,095 & ;ơ, 274 & 39,915 & 62,652 & 98,128 \\
\hline 95 & 16,578 & 26,262 & 41,511 & 65,471 & 103,035 \\
\hline 96 & 17,075 & 27,182 & 43,172 & 68,417 & 108,186 \\
\hline 97 & 17,588 & 28,133 & 44,899 & 71,496 & 113,596 \\
\hline 98 & 18,115 & 29,118 & 46,695 & 74,713 & 119,276 \\
\hline 99 & 18,659 & 30,137 & 48.562 & 78,075 & 125,239 \\
\hline 100 & 19,219 & 31,191 & 50,505 & 81,589 & 131,501 \\
\hline 101 & 19,795 & 32,283 & 52,525 & 85,260 & 138,076 \\
\hline 102 & 20,389 & 33,413 & 54,626 & 89,097 & 144,980 \\
\hline 103 & 21,001 & 34,582 & 56,811 & 93,106 & 152,229 \\
\hline 104 & 21,631 & $3 \check{5}, 793$ & 59,084 & 97,296 & 159,841 \\
\hline 105 & 22,280 & 37,046 & 61,447 & 101,674 & 167,833 \\
\hline
\end{tabular}


TARIF I.

\begin{tabular}{|c|c|c|c|c|c|}
\hline \multirow{2}{*}{ 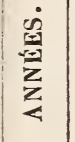 } & \multicolumn{5}{|c|}{ TAUX D'INTÉRÊT. } \\
\hline & 3 р. $0 / 0$ & $3 \frac{1}{2}$ p. 00 & 4 p. $0 / 0$ & $4 \frac{1}{2}$ p. 100 & รั p. 0 \% \\
\hline 106 & 22,948 & 38,342 & 63,905 & 106,250 & 176,224 \\
\hline 107 & 23,636 & 39,684 & 66,461 & 111,031 & 185,035 \\
\hline 108 & 24,346 & 41,073 & 69,119 & 116,027 & 194,287 \\
\hline 109 & 25,076 & 42,511 & 71,884 & 121,249 & 204,002 \\
\hline 110 & 25,828 & 43,999 & 74,760 & 126,70 วั & 214,202 \\
\hline 111 & 26,603 & 45,5339 & 77,750 & 132,406 & 224,912 \\
\hline 112 & 27,401 & 47,132 & 80,860 & 138,365 & 236,157 \\
\hline 113 & 28,223 & 48,782 & 84,094 & 144,591 & 247,965 \\
\hline 114 & 29,070 & 50,489 & 87,458 & 151,098 & 260,363 \\
\hline 115 & 29,942 & 52,256 & 90,957 & $15 \% 7,897$ & 273,382 \\
\hline 116 & 30,840 & 54,085 & 94, วั9 & 165,002 & 287,051 \\
\hline$|117|$ & 31,765 & 55,978 & 98,379 & 172,428 & 301,403 \\
\hline 118 & 32,718 & 57,938 & 102.314 & 180,187 & 316,473 \\
\hline 119 & 33,700 & 59,966 & 106,406 & 188,295 & 332,297 \\
\hline 120 & 34,711 & 62,063 & 110,663 & 196,769 & 348,912 \\
\hline 121 & 35,752 & 64,236 & 115,089 & 205,623 & 366,358 \\
\hline 122 & 36,825 & 66,481 & 119,693 & 214,876 & 384,676 \\
\hline 123 & 37,929 & 68,811 & 124,480 & 224,546 & 403,909 \\
\hline 124 & 39,067 & 71,219 & 129,450 & 234,6 วั0 & 424,105 \\
\hline $11-$ & 40,239 & 73,112 & 134,638 & 245,210 & 445,310 \\
\hline & 41,446 & 76,292 & 140,023 & 256,244 & 467,570 \\
\hline
\end{tabular}


TARIF I.

\begin{tabular}{|c|c|c|c|c|c|}
\hline \multirow{2}{*}{ 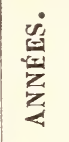 } & \multicolumn{5}{|c|}{ TAUX D'INTÉRÊT. } \\
\hline & 3 p. $0 / 0$. & $3 \frac{1}{2}$ p. $0 / 0$. & 4 p. $0 / 0$. & $4 \frac{1}{2} \mathrm{p} \cdot 0 / 0$ & క p. $0 / 0$ \\
\hline 127 & 42,690 & 78,962 & 145,624 & 267,77 วั & 490,934 \\
\hline 128 & 43,971 & 81,726 & 151,449 & 279,825 & $5 ้ 15,502$ \\
\hline 129 & 45,290 & 84,586 & 157,507 & 292,417 & 541,277 \\
\hline 130 & 46,648 & 87,546 & 163,808 & 306,986 & 568,341 \\
\hline 131 & 48,048 & 90,611 & 170,360 & 319,327 & 596,758 \\
\hline 132 & 49,489 & 93,782 & 177,174 & 333,696 & 626,596 \\
\hline 133 & 74 & 97,064 & 184,261 & 348,713 & 657,926 \\
\hline 134 & 52,503 & 100,462 & 191,632 & 364,40 วั & 690,822 \\
\hline 135 & 54,078 & 103,997 & 199,297 & 380,803 & 725,363 \\
\hline 136 & & 10 & 207,270 & 939 & 31 \\
\hline 137 & 57,372 & 111,384 & 215,560 & 847 & 799,713 \\
\hline 138 & 59,093 & 115,282 & 224,182 & 434,560 & 839,698 \\
\hline 139 & & & 233,149 & 115 & 881,683 \\
\hline 140 & 62,691 & 123,493 & 242,475 & 474,550 & 925,767 \\
\hline 141 & 64,572 & 127,815 & 252,174 & 495,905 & 972,056 \\
\hline 142 & 66,509 & 132,289 & 262,261 & 518,220 & $1020,6 ว$ \\
\hline 143 & 68,505 & 136,919 & 272,752 & 541,545 & 1071,691 \\
\hline 144 & 70,560 & 141,711 & 283,662 & 565,910 & 1125,276 \\
\hline 145 & 72,677 & 6,671 & 295,008 & 591,376 & 1181,540 \\
\hline 146 & 74,857 & 151,804 & 306,809 & 617,988 & 1240,617 \\
\hline 147 & 77,103 & 157,117 & 319,081 & 645,797 & 1302,648 \\
\hline 148 & 79,416 & 162,616 & 331,844 & $674,8 ว ั 8$ & 1367,780 \\
\hline 149 & 81,798 & 168,308 & 345,178 & 705,227 & 1436,169 \\
\hline 150 & 84,252 & 174,199 & $35 ั 8,923$ & 736,962 & 1507,977 \\
\hline
\end{tabular}




\section{TARIF II.}

$$
x=\frac{1}{(1+t)^{n}}
$$

Ce tarif donne les facteurs par lesquels il fant multiplier une somme à percevoir à l'expiration d'un nombre d'années déterminé pour avoir la valeur actuelle de cette somme. 
TARIF $\|$.

\begin{tabular}{|c|c|c|c|c|c|}
\hline \multirow{2}{*}{ 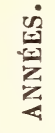 } & \multicolumn{5}{|c|}{ TAUX D'INTÉRÊT. } \\
\hline & 3 p. $0 \mid 0$. & $3 \frac{1}{2}$ p. $0 / 0$. & 4 p. $0 / 0$. & $4 \frac{1}{2}$ p. $0 / 0$ & 5 p. $0,0$. \\
\hline 1 & 0,9709 & 0,9662 & 0,9615 & 0,9569 & 0,952 4 \\
\hline 2 & 0,9426 & 0,9335 & 0,9245 & 0,9157 & 0,9070 \\
\hline 3 & 0,9151 & 0,9019 & 0,8890 & 0,8763 & 0,8638 \\
\hline 4 & 0,8885 & 0,8714 & 0,8548 & 0,8386 & 0,8227 \\
\hline 5 & 0,8626 & 0,8420 & 0,8219 & 0,8024 & 0,7835 \\
\hline 6 & 0,8375 & 0,8135 & 0,7903 & 0,7679 & 0,7462 \\
\hline 7 & 0,8131 & 0,7860 & 0,7599 & 0,7348 & 0,7107 \\
\hline 8 & 0,7894 & 0,7594 & 0,7307 & 0,7032 & 0,6768 \\
\hline 9 & 0,7664 & 0,7337 & 0,7026 & 0,6729 & 0,6446 \\
\hline 10 & 0,7441 & 0,7009 & 0,6756 & 0,6439 & 0,6139 \\
\hline 11 & 0,7224 & 0,6849 & 0,6496 & 0,6162 & 0,5847 \\
\hline 12 & 0,7014 & 0,6618 & 0,6246 & 0,5897 & 0,5568 \\
\hline 13 & 0,6809 & 0,6394 & 0,6006 & 0,5643 & 0,5303 \\
\hline 14 & 0,6611 & 0,6178 & 0.5775 & 0,5400 & 0,5051 \\
\hline 15 & 0,6419 & 0,5969 & 0,5553 & 0,5167 & 0,4810 \\
\hline 16 & 0,6232 & 0,5767 & 0,5339 & 0,4945 & 0,4581 \\
\hline 17 & 0,6050 & 0,5572 & 0,5134 & 0,4732 & 0,4363 \\
\hline 18 & 0,5874 & 0,5384 & 0,4936 & 0,4528 & 0,4155 \\
\hline 19 & 0,5703 & 0,5201 & 0,4746 & 0,4333 & 0,3957 \\
\hline 20 & 0,5537 & 0,5026 & 0,4564 & 0,4146 & 0,3769 \\
\hline 21 & 0,5375 & 0,4856 & 0,4388 & 0,3968 & 0,3589 \\
\hline
\end{tabular}


EN FONDS ET SUPERFICIE.

TARIF II.

\begin{tabular}{|c|c|c|c|c|c|}
\hline \multirow{2}{*}{$\begin{array}{l}\dot{s} \\
\frac{1}{2} \\
z \\
z\end{array}$} & \multicolumn{5}{|c|}{ TAUX D'INTÉRÊT. } \\
\hline & 3 p. $0 / 0$. & $3 \frac{1}{2}$ p. 00. & $4 \mathrm{p} \cdot 0 / 0$ & $4 \frac{1}{2}$ p. $0 / 0$ & 5 р. $0 / 0$. \\
\hline 22 & 0,5219 & 0,4691 & 0,4219 & 0,3797 & 0,3418 \\
\hline 23 & 0,5067 & 0,4538 & 0,4057 & 0,3633 & 0,3256 \\
\hline 24 & 0,4919 & 0,4380 & 0,3901 & 0,3477 & 0,3101 \\
\hline 25 & 0,4776 & 0,4231 & 0,3751 & 0,3327 & 0,2953 \\
\hline 26 & 0,4637 & 0,4088 & 0,3607 & 0,3184 & 0,2812 \\
\hline 27 & 0,4502 & 0,3950 & 0,3468 & 0,3047 & 0,2678 \\
\hline 28 & 0,4371 & 0,3816 & 0,3335 & 0,2916 & 0,2551 \\
\hline 29 & 0,4243 & 0,3687 & 0,3206 & 0,2790 & 0,2429 \\
\hline 30 & 0,4120 & 0,3563 & 0,3083 & 0,2670 & 0,2314 \\
\hline 31 & 0,4000 & 0,3442 & 0,2965 & 0,2555 & 0,2204 \\
\hline 32 & 0,3 & 0,3326 & 0,2851 & 0,2445 & 0,2099 \\
\hline 33 & 0,3770 & 0,3213 & 0,2741 & 0,2340 & 0,1999 \\
\hline 34 & 0,3660 & 0,3105 & 0,2635 & 0,2239 & 0,1903 \\
\hline 35 & 0,3554 & 0,3000 & 0,2534 & 0,2142 & 0,1813 \\
\hline 36 & 0,3450 & 0,2898 & 0,2437 & 0,2050 & 0,1726 \\
\hline 37 & 0,3350 & 0,2800 & 0,2343 & 0,1962 & 0,1644 \\
\hline 38 & 0,3252 & 0,2706 & 0,2253 & 0,1877 & 0,1566 \\
\hline 39 & 0,3157 & 0,2614 & 0,2166 & 0,1797 & 0,1491 \\
\hline 40 & 0,3065 & 0,2526 & 0,2083 & 0,1719 & 0,1420 \\
\hline 41 & 0,2976 & 0,2440 & 0,2003 & 0,1645 & 0,1353 \\
\hline 42 & 0,2890 & 0,2358 & 0,1926 & 0,1574 & 0,1288 \\
\hline
\end{tabular}


TARIF II.

\begin{tabular}{|c|c|c|c|c|c|}
\hline \multirow{2}{*}{ 离 } & \multicolumn{5}{|c|}{ TAUX D'INTÉRÊT. } \\
\hline & 3 p. $0 / 0$. & $3 \frac{1}{2}$ p. $0 / 0$ & $4 \mathrm{p} \cdot 0 / 0$ & $14 \frac{1}{2}$ p. $0 / 0$ & ริ р. $0 / 0$. \\
\hline 43 & 0,2805 & 0,2278 & 0,1852 & 0,1507 & 0,1227 \\
\hline 44 & 0,2724 & 0,2201 & 0,1780 & 0,1442 & 0,1169 \\
\hline 45 & 0,2644 & 0,2127 & 0,1712 & 0,1380 & 0,1113 \\
\hline 46 & 0,2567 & 0,2055 & 0,1646 & 0,1320 & 0,1060 \\
\hline 47 & 0,2493 & 0,1985 & 0,1583 & 0,1263 & 0,1009 \\
\hline 48 & 0,2420 & 0,1918 & 0,1522 & 0,1209 & 0,0961 \\
\hline 49 & 0,2349 & 0,1853 & 0,1463 & 0,1157 & 0,0916 \\
\hline 50 & 0,2281 & 0,1790 & 0,1407 & 0,1107 & 0,0872 \\
\hline 51 & 0,2215 & 0,1730 & 0,1353 & 0,1059 & 0,0830 \\
\hline 52 & 0,2150 & 0,1671 & 0,1301 & 0,1014 & 0,0791 \\
\hline 53 & 0,2087 & 0,1615 & 0,1251 & 0,0970 & 0,0753 \\
\hline 54 & 0,2027 & 0,1560 & 0,1203 & 0,0928 & 0,0717 \\
\hline 55 & 0,1968 & 0,1508 & 0,1156 & 0,0888 & 0,0683 \\
\hline 56 & 0,1910 & 0,1457 & 0,1112 & 0,0850 & 0,0651 \\
\hline 57 & 0,1855 & 0,1407 & 0,1069 & 0,0813 & 0,0620 \\
\hline 58 & 0,1801 & 0,1360 & 0,1028 & 0,0778 & 0,0590 \\
\hline 59 & 0,1748 & 0,1314 & 0,0989 & 0,0745 & 0,0562 \\
\hline 60 & 0,1697 & 0,1269 & 0,0951 & 0,0713 & 0,0535 \\
\hline 61 & 0,1648 & 0,1226 & 0,0914 & 0,0682 & 0,0510 \\
\hline 62 & 0,1600 & 0,1185 & 0,0879 & 0,0653 & 0,0486 \\
\hline 63 & 0,1553 & 0,1145 & 0,0845 & 0,0625 & 0,0462 \\
\hline
\end{tabular}


TARIF II.

\begin{tabular}{|c|c|c|c|c|c|}
\hline \multirow{2}{*}{$\frac{\dot{s}}{2}$} & \multicolumn{5}{|c|}{ TAUX D'INTÉRÊT. } \\
\hline & 2 p. $0 / 0$. & $3 \frac{1}{2}$ p. $0 / 0$. & 4 p. $0 / 0$ & $4 \frac{1}{2}$ p. $0 / 0$ & כ̆ p. $0 / 0$. \\
\hline 64 & 0,1508 & 0,1106 & 0,0813 & 0,0598 & 0,0440 \\
\hline 65 & 0,1464 & 0,1069 & 0,0781 & 0,0572 & 0,0419 \\
\hline 66 & 0,1421 & 0,1033 & 0,0751 & 0,0547 & 0,0399 \\
\hline 67 & 0,1380 & 0,0998 & 0,0722 & 0,0524 & 0,0380 \\
\hline 68 & 0,1340 & 0,0964 & 0,0695 & 0,0501 & 0,0362 \\
\hline 69 & 0,1301 & 0,0931 & 0,0668 & 0,0480 & 0,0345 \\
\hline 70 & 0,1263 & 0,0900 & 0,0642 & 59 & 0,0329 \\
\hline 71 & 0,1226 & 0,0869 & 0,0617 & 0,0439 & 0,0313 \\
\hline 72 & 0,1190 & 0,0840 & 0,0594 & 0,0420 & 0,0298 \\
\hline 73 & 0,1156 & 0,0812 & 0,0571 & 402 & 0,0284 \\
\hline 74 & 0,1122 & 0,0784 & 0,0549 & 0,0385 & 0,0270 \\
\hline 75 & 0,1089 & 0,0758 & 0,0328 & 0,0368 & 0,0257 \\
\hline 76 & 0,1058 & 0,0732 & 0,0507 & 0,0352 & 0,0245 \\
\hline 77 & 0,1027 & 0,0707 & 0,0488 & 0,0337 & 0,0234 \\
\hline 78 & 0,0997 & 0,0683 & 0,0469 & 0,0323 & 0,0222 \\
\hline 79 & 0,0968 & 0,0660 & 0,0451 & 0,0309 & 0,0212 \\
\hline 80 & 0,0940 & 0,0638 & 0,0434 & 0,0295 & 0,0202 \\
\hline 81 & 0,0912 & 0,0616 & 0,0417 & 0,0283 & 0,0192 \\
\hline 82 & 0,0886 & 0,05995 & 0,0401 & 0,0271 & 0,0183 \\
\hline 83 & 0,0860 & 0,0575 & 0,0386 & 0,0259 & 0,0174 \\
\hline 84 & 0,083 วั & 0,0556 & 0,0371 & 0,0248 & 0,0166 \\
\hline
\end{tabular}


TARIF II.

\begin{tabular}{|c|c|c|c|c|c|}
\hline \multirow{2}{*}{ 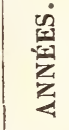 } & \multicolumn{5}{|c|}{ TAUX D'INTÉRÊT. } \\
\hline & 3 p. $0 / 0$. & $3 \frac{1}{2}$ p. $0 / 0$. & 4 p. $0 / 0$ & $4 \frac{1}{2}$ p. $0 / 0$ & อ̃ p. $0 / 0$. \\
\hline 85 & 0,0811 & 0,0537 & 0,0357 & 0,0237 & 0,0158 \\
\hline 86 & 0,0787 & 0,0519 & 0,0343 & 0,0227 & 0,0150 \\
\hline 87 & 0,0764 & 0,0501 & 0,0330 & 0,0217 & 0,0143 \\
\hline 88 & 0,0742 & 0,0484 & 0,0317 & 0,0208 & 0,0136 \\
\hline 89 & 0,0720 & 0,0468 & 0,0309 & 0,0199 & 0,0130 \\
\hline 90 & 0,0699 & 0,0452 & 0,0293 & 0,0190 & 0,0124 \\
\hline 91 & 0,0679 & 0,0437 & 0,0282 & 0,0182 & 0,0118 \\
\hline 92 & 0,0659 & 0,0422 & 0,0271 & 0,0174 & 0,0112 \\
\hline 93 & 0,0640 & 0,0408 & 0,0260 & 0,0167 & 0,0107 \\
\hline 94 & 0,0621 & 0,0394 & 0,0250 & 0,0160 & 0,0102 \\
\hline 95 & 0,0603 & 0,0381 & 0,0241 & 0,0153 & 0,0097 \\
\hline 96 & 0,0586 & 0,0368 & 0,0232 & 0,0146 & 0,0092 \\
\hline 97 & 0,0569 & $0,035 \check{~}$ & 0,0223 & 0,0140 & 0,0088 \\
\hline 98 & 0,0552 & 0,0343 & 0,0214 & 0,0134 & 0,0084 \\
\hline 99 & 0,0536 & 0,0332 & 0,0206 & 0,0128 & 0,0080 \\
\hline 100 & 0,0520 & 0,0321 & 0,0198 & 0,0122 & 0,0076 \\
\hline 101 & 0,0505 & 0,0310 & 0,0190 & 0,0117 & 0,0072 \\
\hline 102 & 0,0490 & 0,0299 & 0,0183 & 0,0112 & 0,0069 \\
\hline 103 & 0,0476 & 0,0289 & 0,0176 & 0,0107 & 0,0066 \\
\hline 104 & 0,0462 & 0,0279 & 0,0169 & 0,0103 & 0,0062 \\
\hline 105 & 0,0449 & 0,0270 & 0,0163 & 0,0098 & 0,0060 \\
\hline
\end{tabular}


TARIF II.

\begin{tabular}{|c|c|c|c|c|c|}
\hline \multirow{2}{*}{$\begin{array}{l}\dot{0} \\
\text { 䆓 } \\
\text { 帘 }\end{array}$} & \multicolumn{5}{|c|}{ TAUX D'INTÉRÊT. } \\
\hline & 3 p. $0 / 0$. & $3 \frac{1}{2}$ p. $0 / 0$ & 4 p. $0 / 0$ & $4 \frac{1}{2}$ p. $0 / 0$ & $5 \mathrm{p.} 0 / 0$. \\
\hline 106 & 0,0436 & 0,0261 & 0,0156 & 0,0094 & 0,0057 \\
\hline 107 & 0,0423 & 0,0252 & 0,0150 & 0,0090 & 0,0054 \\
\hline 108 & 0,0411 & 0,0243 & 0,0145 & 0,0086 & 0,0051 \\
\hline 109 & 0,0399 & 0,0235 & 0,0139 & 0,0082 & 0,0049 \\
\hline 110 & 0,0387 & 0,0227 & 0,0134 & 0,0079 & 0,0047 \\
\hline 111 & 0,0376 & 0,0220 & 0,0129 & 0,0075 & 0,0044 \\
\hline 112 & 0,0365 & 0,0212 & 0,0124 & 0,0072 & 0,0042 \\
\hline 113 & 0,0354 & 0,0205 & 0,0119 & 0,0069 & 0,0040 \\
\hline 114 & 0,0344 & 0,0198 & 0,0114 & 0,0066 & 0,0038 \\
\hline 115 & 0,0334 & 0,0191 & 0,0110 & 0,0063 & 0,0037 \\
\hline 116 & 0,0324 & 0,0185 & 0,0106 & 0,0061 & 0,0035 \\
\hline 117 & 0,0315 & 0,0179 & 0,0102 & 0,0058 & 0,0033 \\
\hline 118 & 0,0306 & 0,0173 & 0,0098 & 0,0055 & 0,0032 \\
\hline 119 & 0,0297 & 0,0167 & 0,0094 & 0,0053 & 0,0030 \\
\hline 120 & 0,0288 & 0,0161 & 0,0090 & 0,0051 & 0,0029 \\
\hline 121 & 0,0280 & 0,0156 & 0,0087 & 0,0049 & 0,0027 \\
\hline 122 & 0,0272 & 0,0150 & 0,0083 & 0,0046 & 0,0026 \\
\hline 123 & 0,0264 & 0,0145 & 0,0080 & 0,0044 & 0,0025 \\
\hline 124 & 0,0256 & 0,0140 & 0,0077 & 0,0043 & 0,0024 \\
\hline 125 & 0,0248 & 0,0136 & 0,0074 & 0,0041 & 0,0022 \\
\hline 126 & 0,0241 & 0,0131 & 0,0071 & 0,0039 & 0,0021 \\
\hline
\end{tabular}


TARIF II.

\begin{tabular}{|c|c|c|c|c|c|}
\hline \multirow{2}{*}{ 窗 } & \multicolumn{5}{|c|}{ TAUX D'INTERÊT. } \\
\hline & 3 p. $0 / 0$ & $3 \frac{1}{2}$ p. $0 / 0$ & 4 p. $0 / 0$ & $4 \frac{1}{2}$ p. $0 / 0$ & కั p. $0 / 0$. \\
\hline 127 & 0,0234 & 0,0127 & 0,0069 & 0,0037 & 0,0020 \\
\hline 128 & 0,0227 & 0,0122 & 0,0066 & 0,0036 & 0,0019 \\
\hline 129 & 0,0221 & 0,0118 & 0,0063 & 0,0034 & 0,0018 \\
\hline 130 & 0,0214 & 0,0114 & 0,0061 & 0,0033 & 0,0018 \\
\hline 131 & 0,0208 & 0,0110 & 0,0059 & 0,0031 & 0,0017 \\
\hline 132 & 0,0202 & 0,0107 & 0,0056 & 0,0030 & 0,0016 \\
\hline 133 & 0,0196 & 0,0103 & 0,0054 & 29 & 0,0015 \\
\hline 134 & 0,0190 & 0,0100 & 0,0052 & 0,0027 & 0,0014 \\
\hline 135 & 0,0185 & 0,0096 & 0,0050 & 0,0026 & 0,0014 \\
\hline 136 & 0,0179 & 0,0093 & 0,0048 & 0,0025 & 0,0013 \\
\hline 137 & 0,0174 & 0,0090 & 0,0046 & 0,0024 & 0,0012 \\
\hline 138 & 0,0169 & 0,0087 & 0,0045 & 0,0023 & 0,0012 \\
\hline 139 & 0,0164 & $0,008 / 4$ & 0,0043 & 0,0022 & 0,0011 \\
\hline 140 & 0,0160 & 0,0081 & 0,0041 & 0,0021 & 0,0011 \\
\hline 14 & 0,0155 & 0,0078 & 0,0040 & 0,0020 & 0,0010 \\
\hline 142 & 0,0150 & 0,0076 & 0,0038 &, 0019 & 0,0010 \\
\hline 143 & 0,0146 & 0,0073 & 0,0037 & 0,0018 & 0,0009 \\
\hline 144 & 0,0142 & 0,0071 & 0,0035 & 0,0018 & 0,0009 \\
\hline 145 & 0,0138 & 0,0068 & 0,0034 & 0,0017 & 0,0008 \\
\hline 146 & 0,0134 & 0,0066 & 0,0033 & 0,0016 & 0,0008 \\
\hline 147 & 0,0130 & 0,0064 & 0,0031 & 0,0015 & 0,0008 \\
\hline 148 & 0,0126 & 0,0061 & 0,0030 & 0, & 0,0007 \\
\hline 149 & 0,0122 & 0,0059 & 0,0029 & 0,0014 & 0,0607 \\
\hline 150 & 0,0119 & 0,0057 & 0,0028 & 0,0014 & 0,0007 \\
\hline
\end{tabular}




\section{TARIF III. \\ $x=\frac{1}{(1+t)^{n}-1}$}

Ce tarif domue les facterns par lesquels il faut multiphier une somme ou

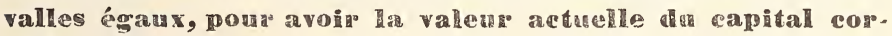
respoudant. 
TARIF III.

\begin{tabular}{|c|c|c|c|c|c|}
\hline \multirow{2}{*}{ 窗 } & \multicolumn{5}{|c|}{ TAUX D’INTÉRÊT. } \\
\hline & 3 p. $0 / 0$. & $3 \frac{1}{2}$ p. $0 / 0$. & $4 \mathrm{p} .0 / 0$ & $4 \frac{1}{2}$ p. $0 / 0$. & ร p. $0 / 0$ \\
\hline 1 & 33,3333 & 28,5714 & 25,0000 & 22,2222 & 20,0000 \\
\hline 2 & 16,4204 & 14,0400 & 12,2549 & 10,8666 & 9,7561 \\
\hline 3 & 10,7843 & 9,1981 & 8,0087 & 7,0838 & 6,3442 \\
\hline 4 & 7,9666 & 6,7786 & 5,8872 & 5,1943 & 4,6402 \\
\hline 5 & 6,2785 & 5,3280 & 4,6157 & 4,0620 & 3,6195 \\
\hline 6 & 5,1532 & 4,3619 & 3,3862 & 3,3084 & 2,9403 \\
\hline 7 & 4,3502 & 3,6727 & 3,1652 & 2,7711 & 2,4564 \\
\hline 8 & 3,7485 & 3,1565 & 2,7132 & 2,3691 & 2,0944 \\
\hline 9 & 3,2811 & 2,7556 & 2,3623 & 2,0572 & 1,8138 \\
\hline 10 & 2,9077 & 2,4355 & 2,0823 & 1,8084 & 1,5901 \\
\hline 11 & 2,6026 & 2,1740 & 1,8537 & 1,6055 & 1,4078 \\
\hline 12 & 2,3515 & 1,9567 & 1,6638 & 1,4370 & 1,2565 \\
\hline 13 & 2,1343 & 1,7732 & 1,5036 & 1,2950 & 1,1291 \\
\hline 14 & 1,9509 & 1,6163 & 1,3667 & 1,1738 & 1,0205 \\
\hline 15 & 1,7922 & 1,4807 & 1,2485 & 1,0692 & 0,9268 \\
\hline 16 & 1,6537 & 1,3624 & 1,1455 & 0,9781 & 0,8454 \\
\hline 17 & 1,5318 & 1,2584 & 1,0550 & 0,8982 & 0,7740 \\
\hline 18 & 1,4236 & 1,1662 & 0,9748 & 0,8275 & 0,7109 \\
\hline 19 & 1,3271 & 1,0840 & 0,9035 & 0,7646 & 0,6549 \\
\hline 20 & 1,2405 & 1,0103 & 0,8395 & 0,7084 & 0,6048 \\
\hline 21 & 1,1624 & 0,9439 & 0,7820 & 0,6578 & 0,5599 \\
\hline
\end{tabular}


TARIF III.

\begin{tabular}{|c|c|c|c|c|c|}
\hline \multirow{2}{*}{ 窻 } & \multicolumn{5}{|c|}{ TAUX D'INTÉRÊT. } \\
\hline & 3 p. $0 / 0$. & $3 \frac{1}{2}$ p. $0 / 0$. & 4 p. $0 / 0$ & $4 \frac{1}{2}$ p. $0 / 0$ & 5 p. $0 / 0$. \\
\hline 22 & 1,0916 & 0,8838 & 0,7300 & 0,6121 & 0,5194 \\
\hline 23 & 1,0271 & 0,8291 & 0,6827 & 0,5707 & 0,4827 \\
\hline 24 & 0,9682 & 0,7792 & 0,6397 & 0,5330 & 0,4495 \\
\hline 25 & 0,9143 & 0,7335 & 0,6003 & 0,4986 & 0,4190 \\
\hline 26 & 0,8646 & 0,6916 & 0,5642 & 0,4671 & 0,3913 \\
\hline 27 & 0,8188 & 0,6529 & 0,5310 & 0,4382 & 0,3658 \\
\hline 28 & 0,7764 & 0,6172 & 0,5003 & 0,4116 & 0,3424 \\
\hline 29 & 0,7371 & 0,5841 & 0,4720 & 0,3870 & 0,3209 \\
\hline 30 & 0,7006 & 0,5535 & 0,4457 & 0,3642 & 0,3010 \\
\hline 31 & 0,6666 & 0,5250 & 0,4214 & 0,3432 & 0,2826 \\
\hline 32 & 0,6349 & 0,4983 & 0,3987 & 0,3236 & 0,2656 \\
\hline 33 & 0,6052 & 0,4735 & 0,3776 & 0,3054 & 0,2498 \\
\hline 34 & 0,5774 & 0,4503 & 0,3579 & 0,2885 & 0,2351 \\
\hline 35 & 0,5513 & 0,4285 & 0,3394 & 0,2727 & 0,2214 \\
\hline 36 & 0,5268 & 0,4081 & 0,3222 & 0,2579 & 0,2087 \\
\hline 37 & 0,5037 & 0,3889 & 0,3060 & 0,2441 & 0,1968 \\
\hline 38 & 0,4820 & 0,3709 & 0,2915 & 0,2311 & 0,1857 \\
\hline 39 & 0,4615 & 0,3539 & 0,2765 & 0,2191 & 0,1753 \\
\hline 40 & 0,4421 & 0,3379 & 0,2631 & 0,2076 & 0,1656 \\
\hline 41 & 0,4237 & 0,3228 & 0,2504 & 0,1969 & 0,1564 \\
\hline 42 & 0,4064 & 0,3085 & 0,2385 & 0,1869 & 0,1479 \\
\hline
\end{tabular}


TARIF III.

\begin{tabular}{|c|c|c|c|c|c|}
\hline \multirow{2}{*}{ 䆓 } & \multicolumn{5}{|c|}{ TAUX D'INTÉRÊT. } \\
\hline & 3 p. $0 / 0$. & $3 \div$ p. $0 / 0$. & 4 p. $0 / 0$. & $4 \frac{1}{2} \mathrm{p} .0 / 0$. & 亏 p. $0 / 0$. \\
\hline 43 & 0,3899 & 0,2950 & 0,2272 & 0,1774 & 0,1399 \\
\hline 44 & 0,3743 & 0,2822 & 0,2166 & 0,1685 & 0,1323 \\
\hline 45 & 0,3595 & 0,2701 & 0,2066 & 0,1600 & 0,1252 \\
\hline 46 & 0,3454 & 0,2586 & 0,1970 & 0,1521 & 0,1186 \\
\hline 47 & 0,3320 & 0,2477 & 0,1880 & 0,1446 & 0,1123 \\
\hline 48 & 0,3193 & 0,2373 & 0,1795 & 0,1375 & 0,1064 \\
\hline 49 & 0,3071 & 0,2275 & 0,1714 & 0,1308 & 0,1008 \\
\hline 50 & 0,2956 & 0,2181 & 0,1637 & 0,1245 & 0,0955 \\
\hline 51 & 0,2845 & 0,2092 & 0,1565 & 0,1185 & 0,0902 \\
\hline 52 & 0,2739 & 0,2007 & 0,1495 & 0,1128 & 0,0859 \\
\hline 53 & 0,2638 & 0,1926 & 0,1430 & 0.1074 & 0,0815 \\
\hline 54 & 0,2542 & 0,1849 & 0,1352 & 0,1023 & 0,0773 \\
\hline 55 & 0,2450 & 0,1775 & 0,1308 & 0,0975 & 0,0733 \\
\hline 56 & 0,2361 & 0,1705 & 0,1251 & 0,0929 & 0,0696 \\
\hline 57 & 0,2277 & 0,1638 & 0,1197 & 0,0885 & 0,0661 \\
\hline 58 & 0,2196 & 0,1574 & 0,1146 & 0,0844 & 0,0627 \\
\hline 59 & 0,2119 & 0,1512 & 0,1097 & 0,0805 & 0,0596 \\
\hline 60 & 0,2044 & 0,1454 & 0,1050 & 0,0768 & 0,0566 \\
\hline 61 & 0,1973 & 0,1398 & 0,1005 & 0,0732 & 0,0537 \\
\hline 62 & 0,1900 & 0,1344 & 0,0964 & 0,0698 & 0,0510 \\
\hline 63 & 0,1839 & 0,1293 & 0,0923 & 0,0666 & 0,0485 \\
\hline
\end{tabular}


TARIF III.

\begin{tabular}{|c|c|c|c|c|c|}
\hline \multirow{2}{*}{ 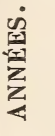 } & \multicolumn{5}{|c|}{ TAUX D'INTÉRÊT. } \\
\hline & 3 p. $0 / 0$. & $3 \frac{1}{2} \mathrm{p} \quad 0,0$ & 4 p. $0 / 0$. & $4 \frac{1}{3}$ p. $0 / 0$ & $5 \mathrm{p} .0 / 0$. \\
\hline 64 & 0,1776 & 0,1244 & 0,0884 & 0,0636 & 0,0461 \\
\hline 65 & 0,1715 & 0.1197 & 0,0847 & 0,0607 & 0,0438 \\
\hline 66 & 0.1657 & 0,1151 & 0,0812 & 0,0579 & 0,0416 \\
\hline 67 & 0,1601 & 0,1108 & 0,0779 & 0,0553 & 0,0395 \\
\hline 68 & 0,1547 & 0,1067 & 0,0746 & 0,0528 & 0,0376 \\
\hline 69 & 0,1495 & 0,1027 & 0,0716 & 0,0504 & 0,0357 \\
\hline 70 & 0,1445 & 0,0989 & 0.0686 & 0,0481 & 0,0340 \\
\hline 71 & 0,1397 & 0,0952 & 0,0658 & 0,0459 & 0,0323 \\
\hline 72 & 0,1351 & 0,0917 & 0,0631 & 0,0439 & 0,0307 \\
\hline 73 & 0,1307 & 0,0883 & 0,0605 & 0,0419 & 0,0292 \\
\hline 74 & 0,1264 & 0,0851 & 0,0581 & 0,0400 & 0,0278 \\
\hline 75 & 0,1223 & 0,0820 & 0,0557 & 0,0382 & 0,0264 \\
\hline 76 & 0,1183 & 0,0790 & 0,0535 & 0,0365 & 0,0251 \\
\hline 77 & 0,1144 & 0,0761 & 0,0513 & 0,0349 & 0,0239 \\
\hline 78 & 0,1107 & 0,0733 & 0,0492 & 0,0333 & 0,0227 \\
\hline 79 & 0,1072 & 0,0707 & 0,0472 & 0,0319 & 0,0216 \\
\hline 80 & 0,1037 & 00681 & 0,0453 & 0,0305 & 0,0206 \\
\hline 81 & 0,1004 & 0,0657 & 0,0435 & 0,0291 & 0,0196 \\
\hline 82 & 0,0972 & 0,0633 & 0,0418 & 0,0278 & 0,0186 \\
\hline 83 & 0,0941 & 0,0610 & 0,0401 & 0,0266 & 0,0177 \\
\hline 84 & 0,0911 & 0,0589 & 0,0385 & 0,0254 & 0,0169 \\
\hline
\end{tabular}


TARIF III.

\begin{tabular}{|c|c|c|c|c|c|}
\hline \multirow{2}{*}{ 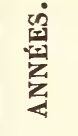 } & \multicolumn{5}{|c|}{ TAUX D'INTÉRÊT. } \\
\hline & 3 p. $0 / 0$. & $3 \frac{1}{2}$ p. $0 / 0$. & 4 p. $0 / 0$. & $4 \div \frac{1}{2}$ p. $0 / 0$ & 5 p. $0 / 0$. \\
\hline 85 & 0,0882 & 0,0568 & 0,0370 & 0,0243 & 0,0167 \\
\hline 86 & 0,0854 & 0,0547 & 0,0355 & 0,0232 & 0,0153 \\
\hline 87 & 0,0827 & 0,0528 & 0,0341 & 0,0222 & 0,0145 \\
\hline 88 & 0,0801 & 0,0509 & 0,0327 & 0,0212 & 0,0138 \\
\hline 89 & 0,0776 & 0,0491 & 0,0314 & 0,0203 & 0,0132 \\
\hline 90 & 0,0752 & 0,0474 & 0,0302 & 0,0194 & 0,0125 \\
\hline 91 & 0,0728 & 0,0457 & 0,0290 & 0,0185 & 0,0119 \\
\hline 92 & 0,0706 & 0,0441 & 0,0278 & 0,0177 & 0,0114 \\
\hline 93 & 0,0684 & 0,0425 & 0,0267 & 0,0170 & 0,0108 \\
\hline 94 & 0,0662 & 0,0410 & 0,0256 & 0,0162 & 0,0103 \\
\hline 95 & 0,0642 & 0,0396 & 0,0247 & 0,0155 & 0,0098 \\
\hline 96 & 0,0622 & 0,0382 & 0,0237 & 0,0148 & 0,0093 \\
\hline 97 & 0,0603 & 0.0368 & 0,0228 &, 0142 & 0,0089 \\
\hline 98 & 0,0584 & 0,0356 & 0,0219 & 0,0136 & 0,0084 \\
\hline 99 & 0,0566 & 0,0343 & 0,0210 & 0,0130 & 0,0080 \\
\hline 100 & 0,0549 & 0,0331 & 0,0202 & 0,0124 & 0,0077 \\
\hline 101 & 0,0532 & 0,0320 & 0,0194 & 0,0119 & 0,0073 \\
\hline 102 & 0,0516 & 0,0308 & 0,0186 & 0,0113 & 0,0069 \\
\hline 103 & 0,0500 & 0,0298 & 0,0179 & 0,0109 & 0,0066 \\
\hline 104 & 0,0485 & 0,0287 & 0,0172 & 0,0104 & 0,0063 \\
\hline 105 & 0,0470 & 0,0277 & 0,0165 & 0,0099 & 0,0060 \\
\hline
\end{tabular}


EN FONDS ET SUPERFICIE.

TARIF III.

\begin{tabular}{|c|c|c|c|c|c|}
\hline \multirow{2}{*}{ 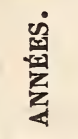 } & \multicolumn{5}{|c|}{ TAUX D'INTÉRÊT. } \\
\hline & 3 p. $0 / 0$ & $3 \frac{1}{2}$ p. $0 / 0$ & 4 p. $0 / 0$ & $4 \frac{1}{2}$ p. $0 / 0$ & 5 p. $0 / 0$. \\
\hline 106 & 0,0456 & 0,0268 & 0,0159 & 0,0095 & 0,0057 \\
\hline 107 & 0,0442 & 0,0258 & 0,0152 & 0,0091 & 0,0054 \\
\hline 108 & 0,0428 & 0,0249 & 0,0147 & 0,0087 & 0,0052 \\
\hline 109 & 0,0415 & 0,0241 & 0,0141 & 0,0083 & 0,0049 \\
\hline 110 & 0,0403 & 0,0232 & 0,0136 & 0,0079 & 0,0047 \\
\hline 111 & 0,0391 & 0,0224 & 0,0130 & 0,0076 & 0,0045 \\
\hline 112 & 0,0379 & 0,0217 & 0,0125 & 0,0073 & 0,0042 \\
\hline 113 & 0,0367 & 0,0209 & 0,0120 & 0,0070 & 0,0040 \\
\hline 114 & 0,0356 & 0,0202 & 0.0116 & 0,0067 & 0,0038 \\
\hline 115 & 0,0346 & 0,0195 & 0,0111 & 0,0064 & 0,0037 \\
\hline 116 & 0.0335 & 0,0188 & 0,0107 & 0,0061 & 0,0035 \\
\hline 117 & 0,0325 & 0,0182 & 0,0103 & 0,0058 & 0,0033 \\
\hline 118 & 0,0315 & 0,0176 & 0,0099 & 0,0056 & 0,0032 \\
\hline 119 & 0,0306 & 0,0170 & 0,0095 & 0,0053 & 0,0030 \\
\hline 120 & 0,0297 & 0,0164 & 0,0091 & 0,0051 & 0,0029 \\
\hline 121 & 0,0288 & 0,0158 & 0,0088 & 0,0049 & 0,0027 \\
\hline 122 & 0,0279 & 0,0153 & 0,0084 & 0.0047 & 0,0026 \\
\hline 123 & 0,0271 & 0,0147 & 0,0081 & 0,0045 & 0,0025 \\
\hline 124 & 0,0263 & 0,0142 & 0,0078 & 0,0043 & 0,0024 \\
\hline 125 & 0,0255 & 0,0139 & 0,0075 & 0,0041 & 0,0022 \\
\hline 126 & 0,0248 & 0,0133 & 0,0072 & 0,0039 & 0,0021 \\
\hline
\end{tabular}


24* TARifs POUR L'estimation EN FONDS ET SUPERFicie.

TARIF III.

\begin{tabular}{|c|c|c|c|c|c|}
\hline \multirow{2}{*}{ 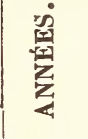 } & \multicolumn{5}{|c|}{ TAUX D'INTERÊT. } \\
\hline & 3 p. $0 / 0$ & $3 \frac{1}{2}$ p. $0 / 0$ & 4 p. 00 & $4 \frac{1}{2}$ p. $0 / 0$. & 5 p. $0 / 0$. \\
\hline 127 & 0,0240 & 0,0128 & 0,0069 & 0,0037 & 0,0020 \\
\hline 128 & 0,0233 & 0,0124 & 0,0066 & 0,0036 & 0,0019 \\
\hline 129 & 0,0226 & 0,0120 & 0,0064 & 0,0034 & 0,0018 \\
\hline 130 & 0,0219 & 0,0116 & 0,0061 & 0,0033 & $0,001 \dot{8}$ \\
\hline 131 & 0,0212 & 0,0112 & 0,0059 & 0,0031 & 0,0017 \\
\hline 132 & 0,0206 & 0,0108 & 0,0057 & 0,0030 & 0,0016 \\
\hline 133 & 0,0200 & 0,0104 & 0,0055 & 0,0029 & 0,0015 \\
\hline 134 & 0,0194 & 0,0100 & 0,0052 & 0,0027 & 0,0014 \\
\hline 135 & 0,0188 & 0,0097 & 0,0050 & 0,0026 & 0,0014 \\
\hline 136 & 0,0183 & 0,0094 & 0,0048 & 0,0025 & 0,0013 \\
\hline 137 & 0,0177 & 0,0091 & 0,0047 & 0,0024 & 0,0012 \\
\hline 138 & 0,0172 & 0,0087 & 0,0045 & 0,0023 & 0,0012 \\
\hline 139 & 0,0167 & 0,0084 & 0,0043 & 0,0022 & 0,0011 \\
\hline 140 & 0,0162 & 0,0082 & 0,0041 & 0,0021 & 0,0011 \\
\hline 141 & 0,0157 & 0,0079 & 0,0040 & 0,0020 & 0,0010 \\
\hline 142 & 0,0153 & 0,0076 & 0,0038 & 0,0019 & 0,0010 \\
\hline 143 & 0,0148 & 0,0074 & 0,0037 & 0,0018 & 0,0009 \\
\hline 144 & 0,0144 & 0,0071 & 0,0035 & 0,0018 & 0,0009 \\
\hline 145 & 0,0139 & 0,0069 & 0,0034 & 0,0017 & 0,0008 \\
\hline 146 & 0,0135 & 0,0066 & 0,0033 & 0,0016 & 0,0008 \\
\hline 147 & 0,0131 & 0,0064 & 0,0031 & 0,0015 & 0,0008 \\
\hline 148 & 0,0127 & 0,0062 & 0,0030 & 0,0015 & 0,0007 \\
\hline 149 & 0,0124 & 0,0060 & 0,0029 & 0,0014 & 0,0607 \\
\hline 150 & 0,0120 & 0,0058 & 0,0028 & 0,0014 & 0,0007 \\
\hline
\end{tabular}




\section{TARIF IV. \\ $x=\frac{1}{t(1+t)^{n-1}}$}

Ce tarif donne les facteurs par lesquels il faut multiplier un revenu annuel et continu que l'on commencera toucher à l'expiration d'un nombre d'années déterminé, pour avoir la valeur actuelle du capital susceptible de produire ce revenu. 
TARIF IV.

\begin{tabular}{|c|c|c|c|c|c|}
\hline \multirow{2}{*}{ 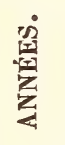 } & \multicolumn{5}{|c|}{ TAUX D'INTÉRÊT. } \\
\hline & 3 p. $0 / 0$ & $3 \div$ p. $0 / 0$ & 4 p. $0 / 0$ & $4 \frac{1}{2}$ p. $0 / 0$ & క p. $0 / 0$. \\
\hline 1 & 33,333 & 28,571 & 25,000 & 22,222 & 20,000 \\
\hline 2 & 32,362 & 27,605 & 24,038 & 21,265 & 19,048 \\
\hline 3 & 31,400 & 26,672 & 23,114 & 20,350 & 18,141 \\
\hline 4 & 30,505 & 25,770 & 22,225 & 19,473 & 17,277 \\
\hline 5 & 29,616 & 24,898 & 21,370 & 18,635 & 16,454 \\
\hline 6 & 28,754 & 24,056 & 20,548 & 17,832 & 15,671 \\
\hline 7 & 27,916 & 23,243 & 19,758 & 17,064 & 14,924 \\
\hline 8 & 27,103 & 22,457 & 18,998 & 16,330 & 14,214 \\
\hline 9 & 26,314 & 21,698 & 18,267 & 15,626 & 13,537 \\
\hline 10 & 25,547 & 20,964 & 17,565 & 14,953 & 12,892 \\
\hline 11 & 24,803 & 20,255 & 16,889 & 14,309 & 12,278 \\
\hline 12 & 24,081 & 19,570 & 16,240 & 13,693 & 11,694 \\
\hline 13 & 23,379 & 18,908 & 15,615 & 13,104 & 11,137 \\
\hline 14 & 22,698 & 18,269 & 15,014 & 12,539 & 10,606 \\
\hline 15 & 22,037 & 17,651 & 14,437 & 11,999 & 10,101 \\
\hline 16 & 21,395 & 17,054 & 13,882 & 11,483 & 9,620 \\
\hline 17 & 20,772 & 16,477 & 13,348 & 10,988 & 9,162 \\
\hline 18 & 20,167 & 15,920 & 12,834 & 10,515 & 8,726 \\
\hline 19 & 19,571 & 15,382 & 12,341 & 10,063 & 8,310 \\
\hline 20 & 19,010 & 14,862 & 11,866 & 9,629 & 7,915 \\
\hline 21 & 18,4506 & 14,359 & 11,410 & 9,214 & 7,538 \\
\hline
\end{tabular}


TARIF IV.

\begin{tabular}{|c|c|c|c|c|c|}
\hline \multirow{2}{*}{ 㦼 } & \multicolumn{5}{|c|}{ TAUX D'INTÉRÊT. } \\
\hline & 3 p. $0 j 0$ & $3 \frac{1}{2} \mathrm{p} \quad 0 / 0$ & 4 p. $0 / 0$. & $4 \frac{1}{2}$ p. $0 / 0$ & อั p. $0 / 0$. \\
\hline 22 & 17,918 & 13,873 & 10,971 & 8,817 & 7,179 \\
\hline 23 & 17,396 & 13,404 & 10,549 & 8,438 & 6,837 \\
\hline 24 & 16,890 & 12,951 & 10,143 & 8,074 & 6,כ๊11 \\
\hline 25 & 16,398 & 12,כૅ13 & 9,753 & 7,727 & 6,201 \\
\hline 26 & 15,920 & 12,090 & 9,378 & 7,394 & 5,906 \\
\hline 27 & 15,456 & 11,681 & 9,017 & 7,076 & 5,625 \\
\hline 28 & 15,006 & 11,286 & 8,670 & 6,771 & 5,357 \\
\hline 29 & 14,569 & 10,904 & 8,337 & 6,479 & 5,102 \\
\hline 30 & 14,145 & 10,536 & 8,016 & 6,200 & 4,859 \\
\hline 31 & 13,733 & 10,179 & 7,708 & 5,933 & 4,628 \\
\hline 32 & 13,333 & 9,835 & 7,411 & 5,678 & 4,407 \\
\hline 33 & 12,945 & 9,503 & 7,126 & 5,433 & 4,197 \\
\hline 34 & 12,568 & 9,181 & 6,852 & ว,199 & 3,997 \\
\hline 35 & 12,201 & 8,871 & 6,589 & 4,97 วั & 3,807 \\
\hline 36 & 11,846 & 8,571 & 6,335 & 4,761 & 3,626 \\
\hline 37 & 11,501 & 8,281 & 6,092 & 4,5506 & 3,453 \\
\hline 38 & 11,166 & 8,001 & 5,857 & 4,360 & 3,289 \\
\hline 39 & 10,841 & 7,730 & ว, 632 & 4,172 & 3,132 \\
\hline 40 & 10,525 & 7,469 & 5,416 & 3,993 & 2,983 \\
\hline 41 & 10,219 & 7,216 & 5,207 & 3,821 & 2,841 \\
\hline 42 & 9,921 & 6,972 & 5,007 & 3,656 & 2,706 \\
\hline
\end{tabular}


TARIF IV.

\begin{tabular}{|c|c|c|c|c|c|}
\hline \multirow{2}{*}{ 窗 } & \multicolumn{5}{|c|}{ TAUX D'INTÉRÊT. } \\
\hline & 3 p. $0 / 0$. & $3 \frac{1}{\vdots}$ p. $0 / 0$. & 4 p. $0 / 0$ & $4 \frac{1}{2}$ p. $0 / 0$. & 5 p. $0 / 0$. \\
\hline 43 & 9,632 & 6,737 & 4,814 & 3,499 & 2,577 \\
\hline 44 & 9,351 & 6,509 & 4,629 & 3,348 & 2,454 \\
\hline 45 & 9,079 & 6,289 & 4,451 & 3,204 & 2,337 \\
\hline 46 & 8,815 & 6,076 & 4,280 & 3,066 & 2,226 \\
\hline 47 & 8,558 & 5,871 & 4,115 & 2,934 & 2,120 \\
\hline 48 & 8,309 & 5,672 & 3,957 & 2,808 & 2,019 \\
\hline 49 & 8,067 & 5,480 & 3,805 & 2,687 & 1,923 \\
\hline 50 & 7,832 & 5,295 & 3,659 & 2,571 & 1,831 \\
\hline 51 & 7,604 & 5,116 & 3,518 & 2,460 & 1,744 \\
\hline 52 & 7,382 & 4,943 & 3,383 & 2,354 & 1,661 \\
\hline 53 & 7,167 & 4,776 & 3,252 & 2,253 & 1,582 \\
\hline 54 & 6,958 & 4,614 & 3,127 & 2,156 & 1,507 \\
\hline 55 & 6,756 & 4,458 & 3,007 & 2,063 & 1,435 \\
\hline 56 & 6,558 & 4,307 & 2,891 & 1,974 & 1,367 \\
\hline 57 & 6,368 & 4,162 & 2,780 & 1,889 & 1,301 \\
\hline 58 & 6,182 & 4,021 & 2,673 & 1,808 & 1,239 \\
\hline 59 & 6,002 & 3,885 & 2,570 & 1,730 & 1,180 \\
\hline 60 & 5,827 & 3,754 & 2,472 & 1,655 & 1.124 \\
\hline 61 & 5,658 & 3,627 & Q, 377 & 1,584 & 1,071 \\
\hline 62 & 5,493 & 3,504 & 2,285 & 1,516 & 1,020 \\
\hline 63 & 5,333 & 3,386 & 2,197 & 1,451 & 0,971 \\
\hline
\end{tabular}


TARIF IV.

\begin{tabular}{|c|c|c|c|c|c|}
\hline \multirow{2}{*}{ 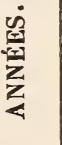 } & \multicolumn{5}{|c|}{ TAUX D'INTÉRÊT. } \\
\hline & 3 p. $0 / 0$ & $3 \frac{1}{2}$ p. $0 / 0$. & 4 p. $0 / 0$ & $4 \frac{1}{2}$ p. $0 / 0$ & $5 \mathrm{p} .0 / 0$. \\
\hline 64 & 5,178 & 3,271 & 2,113 & 1,388 & 0,925 \\
\hline 65 & 5,027 & 3,160 & 2,031 & 1,328 & 0,881 \\
\hline 66 & 4,880 & 3,054 & 1,953 & 1,271 & 0,839 \\
\hline 67 & 4,738 & 2,950 & 1,878 & 1,216 & 0,799 \\
\hline 68 & 4,600 & 2,851 & 1,806 & 1,164 & 0,761 \\
\hline 69 & 4,466 & 2,754 & 1,736 & 1,114 & 0,725 \\
\hline 70 & 4,336 & 2,661 & 1,670 & 1,066 & 0,690 \\
\hline 71 & 4,210 & 2,571 & 1,605 & 1,020 & 0,657 \\
\hline 72 & 4,087 & 2,484 & 1,544 & 0,976 & 0,626 \\
\hline 73 & 3,968 & 2,400 & 1,484 & 0,934 & 0,596 \\
\hline 74 & 3,853 & 2,319 & 1,427 & 0,894 & 0,568 \\
\hline 75 & 3,740 & 2,240 & 1,372 & 0,855 & 0,541 \\
\hline 76 & 3,632 & 2,165 & 1,320 & 0,819 & 0,515 \\
\hline 77 & 3,526 & 2,092 & 1,269 & 0,783 & 0,490 \\
\hline 78 & 3,423 & 2,021 & 1,220 & 0,750 & 0,467 \\
\hline 79 & 3,323 & 1,952 & 1,173 & 0,717 & 0,445 \\
\hline 80 & 3,227 & 1,886 & 1,128 & 0,686 & 0,424 \\
\hline 81 & 3,133 & 1,823 & 1,085 & 0,657 & 0.404 \\
\hline 82 & 3,041 & 1,761 & 1,043 & 0,629 & 0,384 \\
\hline 83 & 2,953 & 1,701 & 1,003 & 0,602 & 0,366 \\
\hline 84 & 2,867 & 1,644 & 0,964 & 0,576 & 0,349 \\
\hline
\end{tabular}


TARIF IV.

\begin{tabular}{|c|c|c|c|c|c|}
\hline \multirow{2}{*}{ 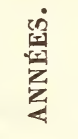 } & \multicolumn{5}{|c|}{ TAUX D'INTÉRÊT. } \\
\hline & 3 p. $0 / 0$. & $3 \frac{1}{2}$ p. $0 / 0$. & 4 p. $0 / 0$ & $4 \div$ p. $0 / 0$ & こ p. $0 / 0$. \\
\hline 85 & 2,783 & 1,588 & 0,927 & 0,551 & 0,332 \\
\hline 86 & 2,702 & 1,535 & 0,891 & 0,527 & 0,316 \\
\hline 87 & 2,623 & 1,483 & 0,857 & 0,504 & 0,301 \\
\hline 88 & 2,547 & 1,433 & 0,824 & 0,483 & 0,287 \\
\hline 89 & 2,473 & 1,384 & 0,793 & 0,462 & 0,273 \\
\hline 90 & 2,401 & 1,337 & 0,772 & 0,442 & 0,260 \\
\hline 91 & 2,331 & 1,292 & 0,733 & 0,423 & 0,248 \\
\hline 92 & 2,263 & 1,248 & 0,705 & 0,405 & 0,236 \\
\hline 93 & 2,197 & 1,206 & 0,677 & 0,387 & 0,225 \\
\hline 94 & 2,133 & 1,165 & 0,651 & 0,371 & 0,214 \\
\hline 95 & 2,071 & 1,126 & 0,626 & 0,355 & 0,204 \\
\hline 96 & 2,012 & 1,088 & 0,602 & 0,339 & 0,194 \\
\hline 97 & 1,952 & 1,05 & 0,579 & 0,325 & 0,185 \\
\hline 98 & 1,895 & 1,016 & 0,557 & 0,311 & 0,176 \\
\hline 99 & 1,840 & 0,981 & 0,535 & 0.297 & 0,168 \\
\hline 100 & 1,786 & 0,948 & 0,515 & 0,285 & 0,160 \\
\hline 101 & 1,734 & 0,916 & 0,495 & 0,272 & 0,152 \\
\hline 102 & 1,684 & 0,885 & 0,476 & 0,261 & 0,145 \\
\hline 103 & 1,635 & 0,855 & 0,458 & 0,249 & 0,138 \\
\hline 104 & 1,587 & 0,826 & 0,440 & 0,239 & 0,131 \\
\hline 105 & 1,541 & 0,798 & 0,423 & 0,228 & 0,125 \\
\hline
\end{tabular}


EN FONDS ET SUPERFICIE.

TARIF IV.

\begin{tabular}{|c|c|c|c|c|c|}
\hline \multirow{2}{*}{ 离 } & \multicolumn{5}{|c|}{ TAUX D'INTÉRÊT. } \\
\hline & 3 p. $0 / 0$. & $3 \frac{1}{2}$ p. $0 / 0$ & 4 p. $0 / 0$ & $4 \frac{1}{2}$ p. $0 / 0$. & 5 p. $0 / 0$. \\
\hline 106 & 1,496 & 0,771 & 0,407 & 0,219 & 0,119 \\
\hline 107 & 1,453 & 0,745 & 0,391 & 0,209 & 0,113 \\
\hline 108 & 1,410 & 0,720 & 0,376 & 0,200 & 0,108 \\
\hline 109 & 1,369 & 0,696 & 0,362 & 0,192 & 0,103 \\
\hline 110 & 1,329 & 0,672 & 0,348 & 0,183 & 0,098 \\
\hline 111 & 1,291 & 0,649 & 0,334 & 0,175 & 0,093 \\
\hline 112 & 1,253 & 0,627 & 0,322 & 0,168 & 0,089 \\
\hline 113 & 1,216 & 0,606 & 0,309 & 0,161 & 0,085 \\
\hline 114 & 1,181 & 0,586 & 0.297 & 0,154 & 0,081 \\
\hline 115 & 1,147 & 0,566 & 0,286 & 0,147 & 0,077 \\
\hline 116 & 1,113 & 0,547 & 0,275 & 0,141 & 0,073 \\
\hline 117 & 1,081 & 0,528 & 0,264 & 0,135 & 0,070 \\
\hline 118 & 1,049 & 0,510 & 0,254 & 0,129 & 0,066 \\
\hline 119 & 1,019 & 0,493 & 0,244 & 0,123 & 0,063 \\
\hline 120 & 0,989 & 0,476 & 0,235 & 0,118 & 0,060 \\
\hline 121 & 0,960 & 0,460 & 0,226 & 0,113 & 0,057 \\
\hline 122 & 0,932 & 0,445 & 0,217 & 0,108 & 0,055 \\
\hline 123 & 0,905 & 0,430 & 0,209 & 0,103 & 0,052 \\
\hline 124 & 0,879 & 0,415 & 0,201 & 0,099 & 0,050 \\
\hline 125 & 0,853 & 0,401 & 0,193 & 0,095 & 0,047 \\
\hline 126 & 0,828 & 0,388 & 0,186 & 0,091 & 0,045 \\
\hline
\end{tabular}


TARIF IV.

\begin{tabular}{|c|c|c|c|c|c|}
\hline \multirow{2}{*}{ 离 } & \multicolumn{5}{|c|}{ TAUX D'INTÉRÊT. } \\
\hline & 3 p. $0 / 0$ & $3 \frac{1}{2}$ p. $0 / 0$. & 4 p. $0 / 0$ & $4 \frac{1}{2}$ p. $0 / 0$ & 丂 p. $0 / 0$ \\
\hline 127 & 0,804 & 0,374 & 0,179 & 0,087 & 0,043 \\
\hline 128 & 0,781 & 0,362 & 0,172 & 0,083 & 0,041 \\
\hline 129 & 0,758 & 0,350 & 0,165 & 0,079 & 0,039 \\
\hline 130 & 0,736 & 0,338 & 0,159 & 0,076 & 0,035 \\
\hline 131 & 0,715 & 0,326 & 0,153 & 0,073 & 0,034 \\
\hline 132 & 0,694 & 0,315 & 0,147 & 0,070 & 0,032 \\
\hline 133 & 0,674 & 0,305 & 0,141 & 0,067 & 0,030 \\
\hline 134 & 0,654 & 0,294 & 0,136 & 0,064 & 0,029 \\
\hline 135 & 0,635 & 0,284 & 0,130 & 0,061 & 0,028 \\
\hline 136 & 0,616 & 0,275 & 0,125 & 0,058 & 0,026 \\
\hline 137 & 0,598 & 0,265 & 0,121 & 0,056 & 0,025 \\
\hline 138 & 0,581 & 0,257 & 0,116 & 0,053 & 0,024 \\
\hline 139 & 0,564 & 0,248 & 0,112 & 0,051 & 0,023 \\
\hline 140 & 0,548 & 0,239 & 0,107 & 0,049 & 0,022 \\
\hline 141 & 0,532 & 0,231 & 0,103 & 0,047 & 0,021 \\
\hline 142 & 0,516 & 0,224 & 0,099 & 0,045 & 0,020 \\
\hline 143 & 0,501 & 0,216 & 0,095 & 0,043 & 0,019 \\
\hline 144 & 0,487 & 0,209 & 0,092 & 0,041 & 0,018 \\
\hline 145 & 0,472 & 0,202 & 0,088 & 0,039 & 0,017 \\
\hline 146 & 0,459 & 0,195 & 0,085 & 0,038 & 0,016 \\
\hline 147 & 0,445 & 0,188 & 0,081 & 0,036 & 0,015 \\
\hline 148 & 0,432 & 0,182 & 0,078 & 0,034 & 0,015 \\
\hline 149 & 0,420 & 0,176 & 0,075 & 0,033 & 0,014 \\
\hline 150 & 0,408 & 0,170 & 0,072 & 0,032 & 0,013 \\
\hline
\end{tabular}




$$
\begin{gathered}
\text { TARIF } \mathbf{V} . \\
x=\frac{1}{t}\left(1-\frac{1}{(1+t)^{n}}\right)
\end{gathered}
$$

Ce tarif donne les factenrs par lesquels il faut multiplier un reveuu à toucher pendant un nombre d'années déterminé, pour avoir la valeur actuelle de tous les revenus réunis. 
TARIF V.

\begin{tabular}{|c|c|c|c|c|c|}
\hline \multirow{2}{*}{ 窗 } & \multicolumn{5}{|c|}{ TAUX D'INTÉRÊT. } \\
\hline & 3 p. $0 / 0$. & $3 \frac{1}{2}$ p. $0 / 0$. & 4 p. $0 / 0$. & $4 \frac{1}{2}$ p. $0 / 0$ & 5 p. $0 / 0$. \\
\hline 1 & 0,971 & 0,966 & 0,962 & 0,957 & 0,952 \\
\hline 2 & 1,913 & 1,900 & $1,886^{\circ}$ & 1,873 & 1,859 \\
\hline 3 & 2,829 & 2,802 & 2,775 & 2,749 & 2,723 \\
\hline 4 & 3,717 & 3,673 & 3,630 & 3,588 & 3,546 \\
\hline 5 & 4,590 & 4,515 & 4,452 & 4,390 & 4,329 \\
\hline 6 & 5,417 & 5,329 & 5.242 & 5,159 & 5,076 \\
\hline 7 & 6,230 & 6,115 & 6,002 & 5,893 & 5,786 \\
\hline 8 & 7,020 & 6,874 & 6,733 & 6,596 & 6,463 \\
\hline 9 & 7,786 & 7,608 & 7,435 & 7,269 & 7,108 \\
\hline 10 & 8,530 & 8,317 & 8,111 & 7,913 & 7,722 \\
\hline 11 & 9,253 & 9,002 & 8,760 & 8,529 & 8,306 \\
\hline 12 & 9,954 & 9,663 & 9,385 & 9,119 & 8,863 \\
\hline 13 & 10,635 & 10,303 & 9,986 & 6,683 & 9,394 \\
\hline 14 & 11,296 & 10,921 & 10,563 & 10,223 & 9,899 \\
\hline 15 & 11,938 & 11,517 & 11,118 & 10,740 & 10,380 \\
\hline 16 & 12,561 & 12,094 & 11,652 & 11,234 & 10,838 \\
\hline 17 & 13,166 & 12,651 & 12,166 & 11,707 & 11,274 \\
\hline 18 & 13,754 & 13,190 & 12,659 & 12,160 & 11,690 \\
\hline 19 & 14,324 & 13,710 & 13,134 & 12,593 & 12,085 \\
\hline 20 & 14,877 & 14,212 & 13,590 & 13,008 & 12,462 \\
\hline 21 & 15,415 & 14,698 & 14,029 & 13,405 & 12,821 \\
\hline
\end{tabular}


EN FONDS ET SUPERFICIE.

TARIF V.

\begin{tabular}{|c|c|c|c|c|c|}
\hline \multirow{2}{*}{ 窗 } & \multicolumn{5}{|c|}{ TAUX D’INTÉRÊT. } \\
\hline & 3 p. $0 / 0$. & $3 \frac{1}{2} \mathrm{p} \quad 0 / 0$. & $4 \mathrm{p}, 0 / 0$ & $4 \frac{1}{2}$ p. $0 / 0$. & ऽ р. $0 / 0$. \\
\hline 22 & 15,937 & 15,167 & 14,451 & 13,784 & 13,163 \\
\hline 23 & 16,444 & 15,620 & 14,857 & 14,148 & 13,489 \\
\hline 24 & 16,936 & 16,058 & 15,247 & 14.495 & 13,799 \\
\hline 25 & 17,413 & 16,482 & 15,622 & 14,828 & 14,094 \\
\hline 26 & 17,877 & 16,890 & 15,983 & 15,147 & 14,375 \\
\hline 27 & 18,327 & 17,285 & 16,330 & 15,451 & 14,643 \\
\hline 28 & 18,764 & 17,667 & 16,663 & 15,743 & 14,898 \\
\hline 29 & 19,188 & 18,036 & 16,984 & 16,022 & 15,141 \\
\hline 30 & 19,600 & 18,392 & 17,292 & 16,289 & 15,372 \\
\hline 31 & 20,000 & 18,736 & 17,588 & 16,544 & 593 \\
\hline 32 & 20,389 & 19,069 & 17,874 & 16,789 & 15,803 \\
\hline 33 & 20,766 & 19,390 & 18,148 & 17,023 & 16,003 \\
\hline 34 & 21,132 & 19,701 & 18,411 & 17,247 & 16,193 \\
\hline 35 & 21,487 & 20,001 & 18,665 & 17,461 & 16,374 \\
\hline 36 & 21,832 & 20,290 & 18,908 & 17,666 & 16,547 \\
\hline 37 & 22,167 & 20,571 & 19,143 & 17,862 & 16,711 \\
\hline 38 & 22,492 & 20,841 & 19,368 & 18,050 & 16,868 \\
\hline 39 & 22,808 & 21,102 & 19,584 & 18,230 & 17,017 \\
\hline 40 & 23,115 & 21,355 & 19,793 & 18,402 & 17,159 \\
\hline 41 & 23,412 & 21,599 & 19,993 & 18,566 & 17,294 \\
\hline 42 & 23,701 & 21,835 & 20,186 & 18,724 & 17,423 \\
\hline
\end{tabular}


TARIF $V$.

\begin{tabular}{|c|c|c|c|c|c|}
\hline \multirow{2}{*}{ 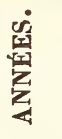 } & \multicolumn{5}{|c|}{ TAUX D'INTÉRÊT. } \\
\hline & 3 p. $0 / 0$. & $3 \frac{1}{2}$ p. $0 / 0$. & 4 p. $0 / 0$ & $4 \frac{1}{2}$ p. $0 / 0$ & כ p. $0 / 0$. \\
\hline 43 & 23,982 & 22,063 & 20,371 & 18,874 & 17,546 \\
\hline 44 & 24,254 & 22,283 & 20,549 & 19,018 & 17,663 \\
\hline 45 & 24,519 & 22,495 & 20,720 & 19,156 & 17,774 \\
\hline 46 & 24,775 & 22,701 & 20,885 & 19,288 & 17,880 \\
\hline 47 & 25,025 & 22,899 & 21,043 & 19,415 & 17,981 \\
\hline 48 & 25,267 & 23,091 & 21,195 & 19,536 & 18,077 \\
\hline 49 & 25,502 & 23,277 & 21,341 & 19,651 & 18,169 \\
\hline 50 & 25,730 & 23,456 & 21,482 & 19,762 & 18,256 \\
\hline 51 & 25,951 & 23,629 & 21,617 & 19,868 & 18,339 \\
\hline 52 & 26,166 & 23,796 & 21,748 & 19,969 & 18,418 \\
\hline 53 & 26,375 & 23,957 & 21,873 & 20,066 & 18,493 \\
\hline 54 & 26,578 & 24,113 & 21,993 & 20,159 & 18,565 \\
\hline 55 & 26,774 & 24,264 & 22,109 & 20,248 & 18,633 \\
\hline 56 & 26,965 & 24,410 & 22,220 & 20,333 & 18,699 \\
\hline 57 & 27,151 & 24,550 & 22,327 & 20,414 & 18,761 \\
\hline 58 & 27,331 & 24,686 & 22,430 & 20,492 & 18,820 \\
\hline 59 & 27,506 & 24,818 & 22,528 & 20,567 & 18,876 \\
\hline 60 & 27,676 & 24,945 & 22,623 & 20,638 & 18,929 \\
\hline 61 & 27,840 & 25,067 & 22,715 & 20,706 & 18,980 \\
\hline 62 & 28,000 & 25,186 & 22,803 & 20,772 & 19,029 \\
\hline 63 & 28,156 & 25,300 & 22,887 & 20,834 & 19,075 \\
\hline
\end{tabular}


TARIF $\mathrm{V}$.

\begin{tabular}{|c|c|c|c|c|c|}
\hline \multirow{2}{*}{ 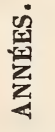 } & \multicolumn{5}{|c|}{ TAUX D'INTÉRÊT. } \\
\hline & 3 p. $0 / 0$. & $3 \frac{1}{2}$ p. $0 / 0$. & 4 p. $0 / 0$ & $4 \frac{1}{2}$ p. $0 / 0$ & 5 p. $0 / 0$. \\
\hline 64 & 28,306 & 25,411 & 22,969 & 20,894 & 19,119 \\
\hline 65 & 28,453 & 25,518 & 23,047 & 20,951 & 19,161 \\
\hline 66 & 28,595 & 25,621 & 23,122 & 21,006 & 19,201 \\
\hline 67 & 28,733 & 25,721 & 23,194 & 21,058 & 19,239 \\
\hline 68 & 28,867 & 25,817 & 23,264 & 21,108 & 19,275 \\
\hline 69 & 28,997 & 25,910 & 23,330 & 21,156 & 19,310 \\
\hline 70 & 29,123 & 26,000 & 23,395 & 21,202 & 19,343 \\
\hline 71 & 29,246 & 26,087 & 23,456 & 21,246 & 19,374 \\
\hline 72 & 29,365 & 26,171 & 23,516 & 21,288 & 19,404 \\
\hline 73 & 29,481 & 26,252 & 23,573 & 21,328 & 19,432 \\
\hline 74 & 29,593 & 26,331 & 23,628 & 21,367 & 19,459 \\
\hline 75 & 29,702 & 26,407 & 23,680 & 21,404 & 19,485 \\
\hline 76 & 29,808 & 26,480 & 23,731 & 21,439 & 19,509 \\
\hline 77 & 29,910 & 26,551 & 23,780 & 21,473 & 19,533 \\
\hline 78 & 30,010 & 26,619 & 23,827 & 21,505 & 19,555 \\
\hline 79 & 30,107 & 26,685 & 23,872 & 21,536 & 19.576 \\
\hline 80 & 30,201 & 26,749 & 23,915 & 21,565 & 19,596 \\
\hline 81 & 30,292 & 26,810 & 23,957 & 21,594 & 19,616 \\
\hline 82 & 30,381 & 26,870 & 23,997 & 21,621 & 19,634 \\
\hline 83 & 30,467 & 26,927 & 24,036 & 21,647 & 19,651 \\
\hline 84 & 30,550 & 26,983 & 24,073 & 21,671 & 19,668 \\
\hline
\end{tabular}


TARIF V.

\begin{tabular}{|c|c|c|c|c|c|}
\hline \multirow{2}{*}{ 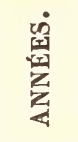 } & \multicolumn{5}{|c|}{ TAUX D'INTÉRÊT. } \\
\hline & 3 p. $0 / 0$ & $3 \frac{1}{2}$ p. $0 / 0$ & 4 p. $0 / 0$. & $4 \frac{1}{2}$ p. $0 / 0$ & 与 p. $0 / 0$. \\
\hline 85 & 30,631 & 27,037 & 24,109 & 21,695 & 19,684 \\
\hline 86 & 30,710 & 27,089 & 24,143 & 21,718 & 19,699 \\
\hline 87 & 30,786 & 27,139 & 24,176 & 21,740 & 19,713 \\
\hline 88 & 30,860 & 27,234 & 24,207 & 21,760 & 19,727 \\
\hline 89 & 30,932 & 27,279 & 24,238 & 21,780 & 19,740 \\
\hline 90 & 31,002 & 27,279 & 24,267 & 21,799 & 19,752 \\
\hline 91 & 31,070 & 27,323 & 24,295 & 21,817 & 19,764 \\
\hline 92 & 31,136 & 27,365 & 24,323 & 21,835 & 19,775 \\
\hline 93 & 31,200 & 27,406 & 24,349 & 21,852 & 19,786 \\
\hline 94 & 31,262 & 27,445 & 24,374 & 21,868 & 19,796 \\
\hline 95 & 31,323 & 27,483 & 24,398 & 21,883 & 19,806 \\
\hline 96 & 31,381 & 27,520 & 24,421 & 21,897 & 19,815 \\
\hline 97 & 31,438 & 27,556 & 24,443 & 21,911 & 19,824 \\
\hline 98 & 31,493 & 27,590 & 24,465 & 21,925 & 19,832 \\
\hline 99 & 31,547 & 27,623 & 24,485 & 21,938 & 19,840 \\
\hline 100 & 31,599 & 27,655 & 24,505 & 21,950 & 19,848 \\
\hline 101 & 31,649 & 27,686 & 24,524 & 21,962 & 19,855 \\
\hline 102 & 31,698 & 27,716 & 24,542 & 21,973 & 19,862 \\
\hline 103 & 31,746 & 27,745 & 24,560 & 21,984 & 19,869 \\
\hline 104 & 31,792 & 27,773 & 24,577 & 21,994 & 19,875 \\
\hline 105 & 31,837 & 27,800 & 24,593 & 22,004 & 19,881 \\
\hline
\end{tabular}


TARIF V.

\begin{tabular}{|c|c|c|c|c|c|}
\hline \multirow{2}{*}{ 㴓 } & \multicolumn{5}{|c|}{ TAUX D'INTÉRÊT. } \\
\hline & 3 p. $0 / 0$. & $3 \frac{1}{2}$ p. $0 / 0$ & 4 p. $0 / 0$ & $4 \frac{1}{2}$ p. $0 / 0$ & 5 p. $0 / 0$. \\
\hline 106 & 31,881 & 27,826 & 24,609 & 22,013 & 19,887 \\
\hline 107 & 31,923 & 27,851 & 24,624 & 22,022 & 19,892 \\
\hline 108 & 31,964 & 27,876 & 24,638 & 22,031 & 19,897 \\
\hline 109 & 32,004 & 27,899 & 24,652 & 22,039 & 19,902 \\
\hline 110 & 32,043 & 27,922 & 24,666 & 22,047 & 19,907 \\
\hline 111 & 32,080 & 27,944 & 24,678 & 22,054 & 19,911 \\
\hline 112 & 32,117 & 27,965 & 24,691 & 22,062 & 19,915 \\
\hline 113 & 32,152 & 27,986 & 24,703 & 22,069 & 19,919 \\
\hline 114 & 32,187 & 28,006 & 24,714 & 22,075 & 19,923 \\
\hline 115 & 32,220 & 28,025 & 24,725 & 22,081 & 19,927 \\
\hline 116 & 32,252 & 28,043 & 24,736 & 22,088 & 19,930 \\
\hline 117 & 32,284 & 28,061 & 24,746 & 22,093 & 19,934 \\
\hline 118 & 32,315 & 28,078 & 24,756 & 22,099 & 19,937 \\
\hline 119 & 32,344 & 28,095 & 24,765 & 22,104 & 19,940 \\
\hline 120 & 32,373 & 28,111 & 24,774 & 22,109 & 19,943 \\
\hline 121 & 32,401 & 28,127 & 24,783 & 22,114 & 19,945 \\
\hline 122 & 32,428 & 28,142 & 24,791 & 22,119 & 19,948 \\
\hline 123 & 32,455 & 28,156 & 24,799 & 22,123 & 19,950 \\
\hline 124 & 32,480 & 28,170 & 24,807 & 22,127 & 19,953 \\
\hline 125 & 32,505 & 28,184 & 24,814 & 22,132 & 19,955 \\
\hline 126 & 32,529 & 28.197 & 24,821 & 22,135 & 19,957 \\
\hline
\end{tabular}


40* TARIFS POUR L'ESTIHATION EN FONDS ET SUPERFICIE.

TARIF V.

\begin{tabular}{|c|c|c|c|c|c|}
\hline \multirow{2}{*}{ 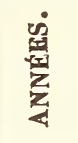 } & \multicolumn{5}{|c|}{ TAUX D'INTÉRÊT. } \\
\hline & 3 p. $0 / 0$. & $3 \frac{1}{2}$ p. $0 / 0$. & 4 p. $0 ; 0$ & $4 \frac{1}{2}$ p. $0 / 0$ & ร p. $0 / 0$. \\
\hline 127 & 32,551 & 28,212 & 24,828 & 22,139 & 19,959 \\
\hline 128 & 32,575 & 28,222 & 24,835 & 22,143 & 19,961 \\
\hline 129 & 32,597 & 28,234 & 24,841 & 22,146 & 19,963 \\
\hline 130 & 32,619 & 28,245 & 24,847 & 149 & 19,0 \\
\hline 131 & 32,640 & 28,256 & 24,853 & 22,153 & 19,967 \\
\hline 132 & 32,660 & 28,267 & 24,859 & 22,156 & 19,968 \\
\hline 133 & 32,679 & 28,277 & 24,864 & 22,158 & 19,97 \\
\hline 134 & 32,698 & 28,287 & 24,870 & 22,161 & 19,971 \\
\hline 135 & 32,717 & 28,297 & 24,875 & 22,164 & 19,972 \\
\hline 136 & 32,735 & 28,306 & 24,879 & 22,166 & 19,97 \\
\hline 137 & 32,752 & 28,315 & 24,884 & 22,169 & 19,9 \\
\hline 138 & 32 & 28,324 & 24,888 & 22,171 & 19,97 \\
\hline 139 & 32,786 & 28,332 & $2 / 4,893$ & 22,173 & 19 \\
\hline 140 & 32,802 & 28,340 & 24,897 & 22,175 & 19,978 \\
\hline 141 & 32,817 & $989 / 8$ & 24 & 77 & 19,979 \\
\hline 142 & 32,832 & 28,355 & 24,905 & 2,179 & 980 \\
\hline 143 & 23,847 & 28,363 & 24,908 & 22,181 & 19,981 \\
\hline 144 & 32,861 & 28,370 & 24,912 & 22,183 & 19,982 \\
\hline 145 & 32,875 & 28,377 & 24,915 & 22,185 & 19,983 \\
\hline 146 & 32,888 & 28,383 & 24,919 & 22,186 & 19,984 \\
\hline 147 & 32,901 & 28,390 & 24,922 & 22,188 & 19,98 \\
\hline 148 & 32,914 & & 24,925 & 22,189 & 19,985 \\
\hline 149 & 32,926 & 28,402 & 24,928 & 22,191 & 19,986 \\
\hline 150 & 32,938 & 28,407 & 24,930 & 22,192 & 19,987 \\
\hline
\end{tabular}




\title{
TABLE DES MATIËRES.
}

\author{
PREMIÉRE PARTIE.
}

Exploitation, débit et conservation des bois.

CHAPITRE PREMIER.

Abatage des bois.

Article I.

Abatage des bois dans les taillis..............

Article II.

Abatage des bois dans les futaies............. 3

Article III.

Abatage des bois dans les coupes d'amélioration...... 6

Article IV.

Des bois qui s'exploitent avec la faculté d'écorcer....

\section{CHAPITRE DEUXIEMME.}

anode die flébit, fle mesurage et de vente des hois de chaufigge.

Article I.

Gẻnéralités........................... 21 


\section{TABLE DES MATIËRES.}

Article II.

Bois de corde.

§I. Découpe et dressage des bois de corde ........ 21

$\S$ II. Mode de mesurage et de vente des bois de corde.. 25

$\S$ III. Qualités et défauts des bois de corde........ 30

Article III.

Façonnage des fagots et bourrées............. 33

Article IV.

Bois à charbon.

$\S$ I. Carbonisation du bois en forêt........... 34

$\S$ II. Mode de mesurage et de vente du charbon ..... 39

\section{Article V.}

Des facteurs de conversion du mètre cube plein au volume empilé en bois de feu............. 42

\section{GHAPITRE TROISIĖME.}

Mode de débit, de mesurage et de vente des bois d'œenvre.

\section{Article !.}

Généralités.... . . . . . . . . . . . . . 49

\section{Article II.}

Mode de débit, de cubage et de vente des bois de service.

§ I. Débit des bois de service............... 51

$\S$ II. Mode de mesurage, de cubage et de vente des bois ronds ou en grume .................. 56

$\S$ III. Mode de mesurage, de cubage et de vente des bois équarris ou carrés................... 66 


\section{Article III.}

Mode de débit, de mesurage et de vente des bois de travail ou d'industrie façonnés dans les coupes.

§ 1. Sciages de chêne ................. 70

$\S$ II. Sciages de hêtre .................. 78

$\S$ III. Sciages de sapin................... 81

$\S$ IV. Bois de fente.................. 90

\section{GHAPITRE QUATRIĖME.}

Rois de manoำ.

\section{Article I.}

Classement, mesurage et cubage des bois de chêne.

$\S$ I. Exercice du martelage dans les forêts domaniales...

$\S$ II. Nomenclature et configuration des pièces de bois de chêne propres aux constructions navales ........ 108

$\S$ IIl. Mode de mesurage, de cubage et de classement des bois de marine abaltus, équarris ou en grume ..... 116

$\S I V$. Mode de classement des arbres sur pied en signaux et espèces de marine d'après leurs formes et leurs di-

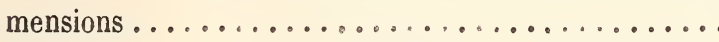

Artiche II.

Emploi des bois de chêne.

$\S$ I. Emploi des pièces de quille, étambots, courbes d'étambot, étraves et brions . . . . . . . . . . . . . 129

$\S$ II. Emploi des varangués, genoux et allonges...... 132

$\S$ III. Emploi des baux, courbes de pont, guirlandes et courbes de jottereau................ 136

§ IV. Emploi des plançons, pièces de tour, préceintes et bois à deux bouges ................... 142

$\S \mathrm{V}$. Emploi des bittes, mèches de gouvernail, jas d'ancre. 146 
TABLE DES MATIÈRES.

Article III.

Bois résineux.

$\S$ I. Des résineux qui servent aux constructions navales

$\S$ II. Proportions et classement de la mâture par degré de qualité et d'utilité ................. 152

$\S$ III. Précis des règles générales de recette des bois de mâture... ..................... 152

Article IV.

Emploi spécial des bois de mâture

Article V.

Des bois employés aux emménagements et à l'armement des vaisseaux...................... 159

\section{GHAPITRE CINQUIĖME.}

Des qualités et défauts des bois d'ouvre.

Article I.

De la structure des bois.................... 169

\section{Article II.}

Des causes qui influent sur la qualité des bois de même espèce, et des caractères qui servent à la faire reconnaître.......................... 167

\section{ArTicle III.}

Des principaux vices ou défauts qui se rencontrent dans les bois d'œurre.

§ I. Généralités...................... 177

$\S$ II. De la torsion des fibres................ 178 
$\S$ III. De la gélivure.................... 179

$\S$ IV. De la roulure $\ldots \ldots \ldots \ldots \ldots \ldots \ldots \ldots \ldots$

$\S$ V. Cadranure et pourriture.............. 184

$\S$ VI. Grisette, nœuds, taches du bois........... 188

$\S$ VII. Lunure ........................ 194

$\S$ VIIl. Frotture, entr'écorce, trous de vers........ 203

\section{CHAPITRE SIXIEME.}

De la conservation thes bois d'oeuvie.

\section{Article I.}

Des conditions dans lesquelles les bois sont employés ou mis en œuvre.. ................... 207

\section{Article II.}

Des procédés ordinaires de conservation des bois d'œuvre. 209

Article III.

De la conservation des bois d'œurre par le procédé du docteur Boucherie.................. 213

DEUXIEMME PARTIE.

Estimation et vente des bois sur pied. GHAPITRE PREMIER.

De l'estimation er matièrec des bois sur pied.

\section{Article I.}

Généralités......................... 
TABLE DES MATIÉRES.

Article II.

De l'estimation par le comptage et le cubage individuel des arbres.

$\S$ I. Exposé de la méthode............... 227

$\S$ II. Mesurage des dimensions de İa tige des arbres.... 228

$\S$ III. Détermination du volume réel des arbres types .. 23こ

$\S$ IV. Estimation des arbres abandonnés à l'exploitation dans les coupes de futaie ou de taillis composé..... 240

Article III.

Estimation à vue d'œil et par pied d'arbre.. . . . . . 246

\section{Article IV.}

Estimation à vue d'œil, par hectare ou par virée. . . 2 2こ0

Article V

De l'estimation en matière des bois sur pied par places d'essai. .......................... 254

Article VI.

De l'estimation des bois d'œurre sur pied en produits façonnés.......................

\section{CHAPITRE DEUXIÈME.}

De l'estimation en argent des bois ì vendre sur pied.......................... 2 วั 8

\section{CHAPITRE TROISIÈME.}

Des différentes opérations à faire dans les coupes, selon le mode d'adjudication et d'exploitation des produits.

Anticle I.

De la vente des bois sur pied et des opérations préparatoires qu'elle exige.................. 263 
TABLE DES MATIÈRES.

Article II.

De la vente des bois façonnés par économie ou par entreprise, et du mode d'exploitation de ces produits.....

Article III.

De la vente des bois sur pied à tant l'unité de produits façonnés........................ 270

Estimation de la valeur des forêts en fonds et superficie. CHAPITRE PREMIER.

Exposé des principes.

Article I.

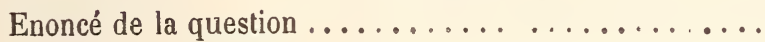

Article II.

Choix du taux d'intérêt ou de placement..........

Akticle IlI.

Recherche du revenu.

$\S$ I. Du mode de constitution et de production des pro-

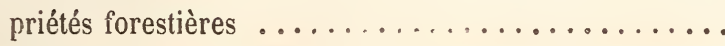

$\S$ II. Fixation du terme de l'exploitabilité commerciale et du revenu correspondant d'un bois peuplé d'arbres du même âge (Taillis simples, futaies régulières) ...

$\S$ III. Fixation du terme de l'exploitabilité commerciale et du revenu à cet âge d'un peuplement composé de bois d’âges différents (Taillis composés, futaies jardinées, etc.) $\ldots \ldots \ldots \ldots \ldots \ldots \ldots \ldots \ldots \ldots \ldots$

CHAPITRE DEUXIÈME,

Aplication générale des principes en matière d'estimation ale forêts en fonds et superficie...... 307 
TABLE DES MATIÈRES.

\section{CHAPITRE TROISIEME.}

Exécution des calculs.

Article I.

Observation générale $\ldots \ldots \ldots \ldots \ldots \ldots \ldots \ldots \ldots$

Article II.

Formation et emploi du Tarif $1 \ldots \ldots \ldots \ldots \ldots 317$

Ar ticlb III.

Formation et emploi du Tarif II ... . . ....... 318

Article IV.

Formation et emploi du Tarif III............. . 319

Article V.

Formation et emploi du Tarif IV........... 320

Article VI.

Formation et emploi du Tarif V ............. 322

CHAPITRE QUATRIĖNE.

Problèmes sur l'estimation des forêts eu fonds et superficie.

Problème $\mathrm{n}^{n}$ 1. - Vente de superficie .......... 32.

- $\quad \mathrm{n}^{0}$ 2. - Vente de fonds et superficie..... 327

- $\mathrm{n}^{0}$ 3. - Echange ................ 330

- $n^{0} 4$. - Partage................. 333

- $\mathrm{n}^{0} 5$. - Vente de fords et superficie avec faculté de défricher. . . . . . . . . . . . . . 340

Problème $n^{0} 6$. - Vente de fonds et superficie avec faculté de défricher ..................... 345

Problème $n^{0}$ 7. - Cantonnement de droits d'usage .... 351

$\begin{array}{llllll} & \text { n } 8 . & - & - & - & \ldots\end{array}$

Tastifs.

FIN DE LA TABLE DES MATIÈRES. 
PL.I.
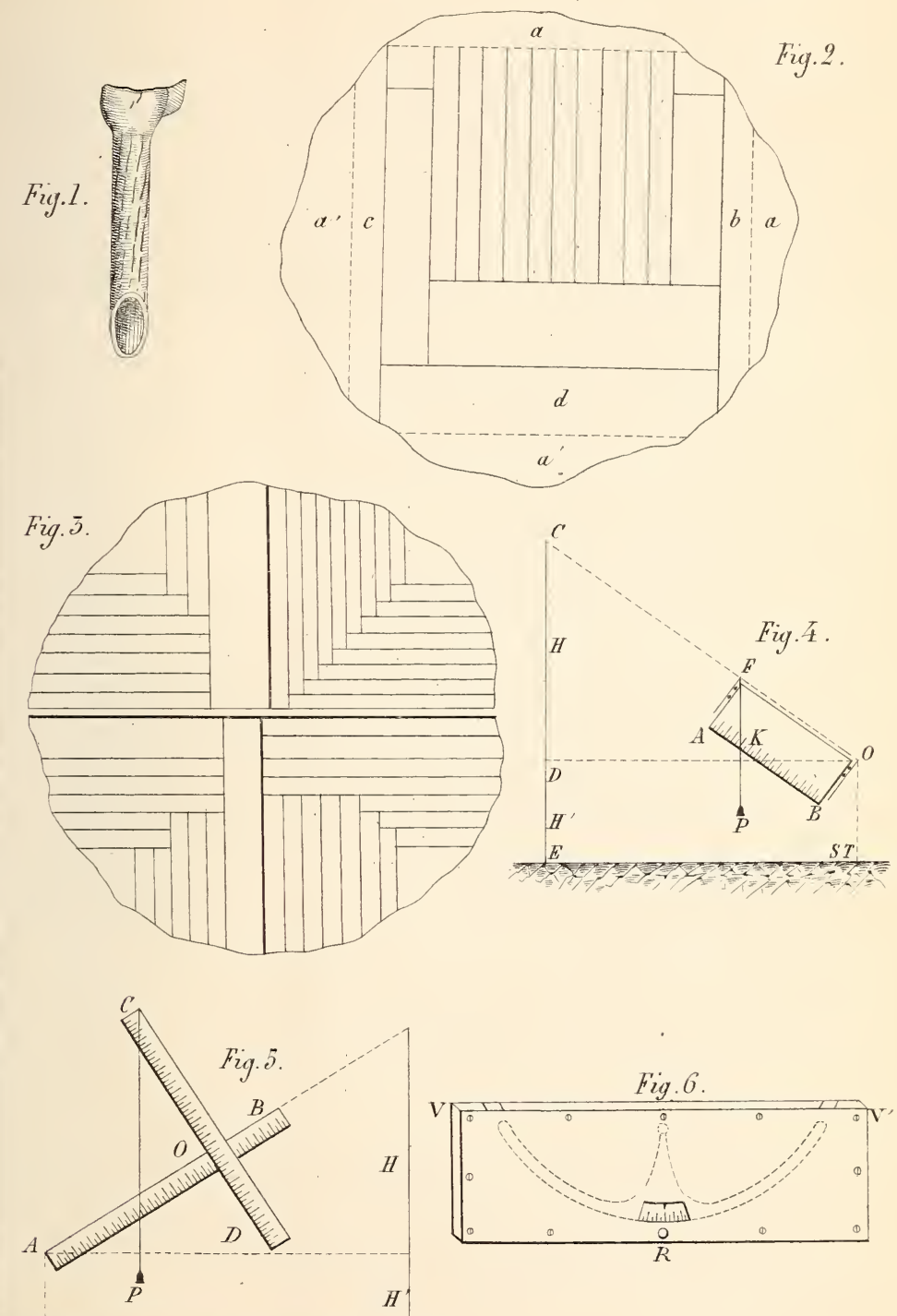

ST 

PL. II.

Bois droits.

Quille, Étambot, Weiche de gonvernail.

\begin{tabular}{|lll|}
\hline & \\
\hline & $E T$ & $M$
\end{tabular}

Plancon.

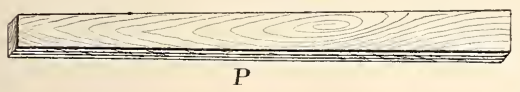

Demi-Ban.
Bitte.

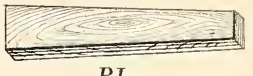

$B I$

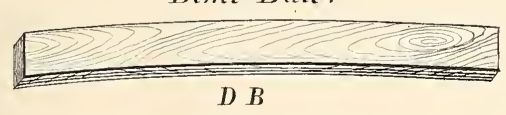

Ban, Barrot de gaillard.

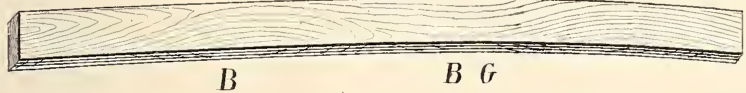

\section{Bois à une courbure.}

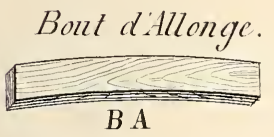

Jas d'ancre, demi-varangne.

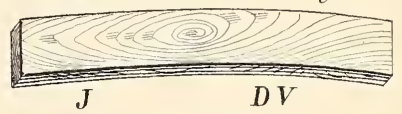

Varangue plate, Préecinte de tour.
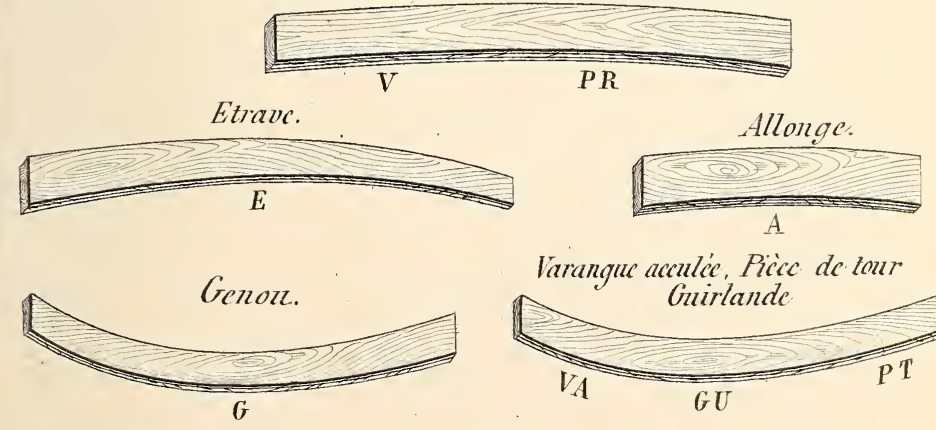

Varangue acculce, Picice de tour

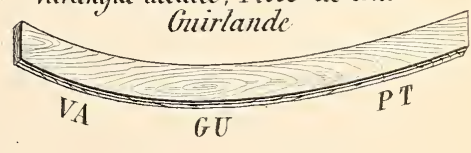





\section{Bois a deux courbures.}
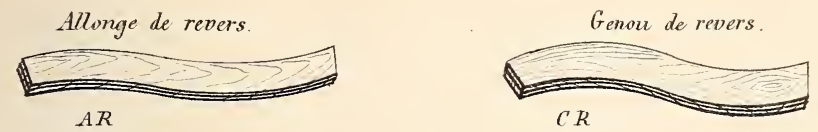

Buis à deux bonges.

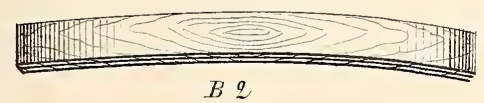

Petits bois:

Bois de barque.

$B B$

Coumbes.

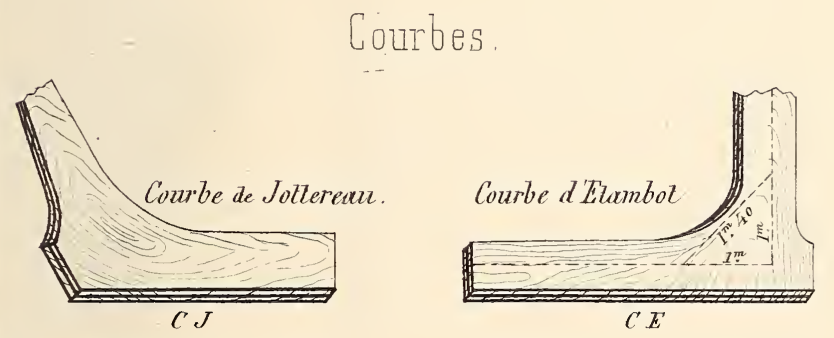

Bois de chaloupe.
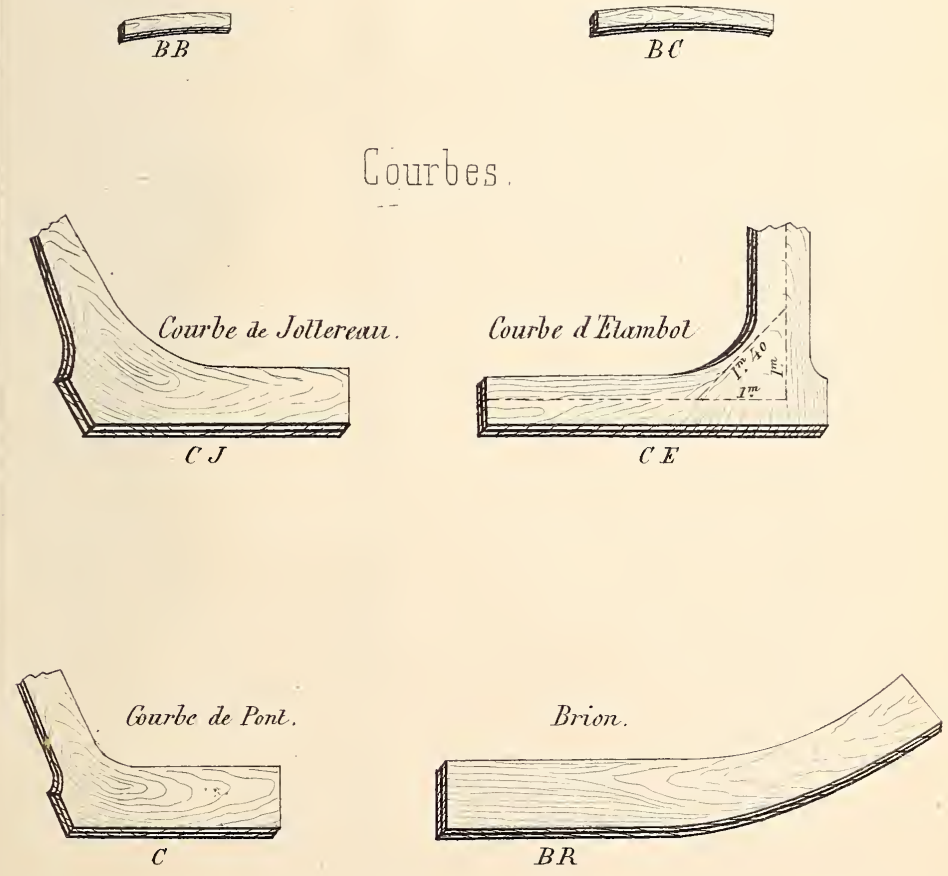



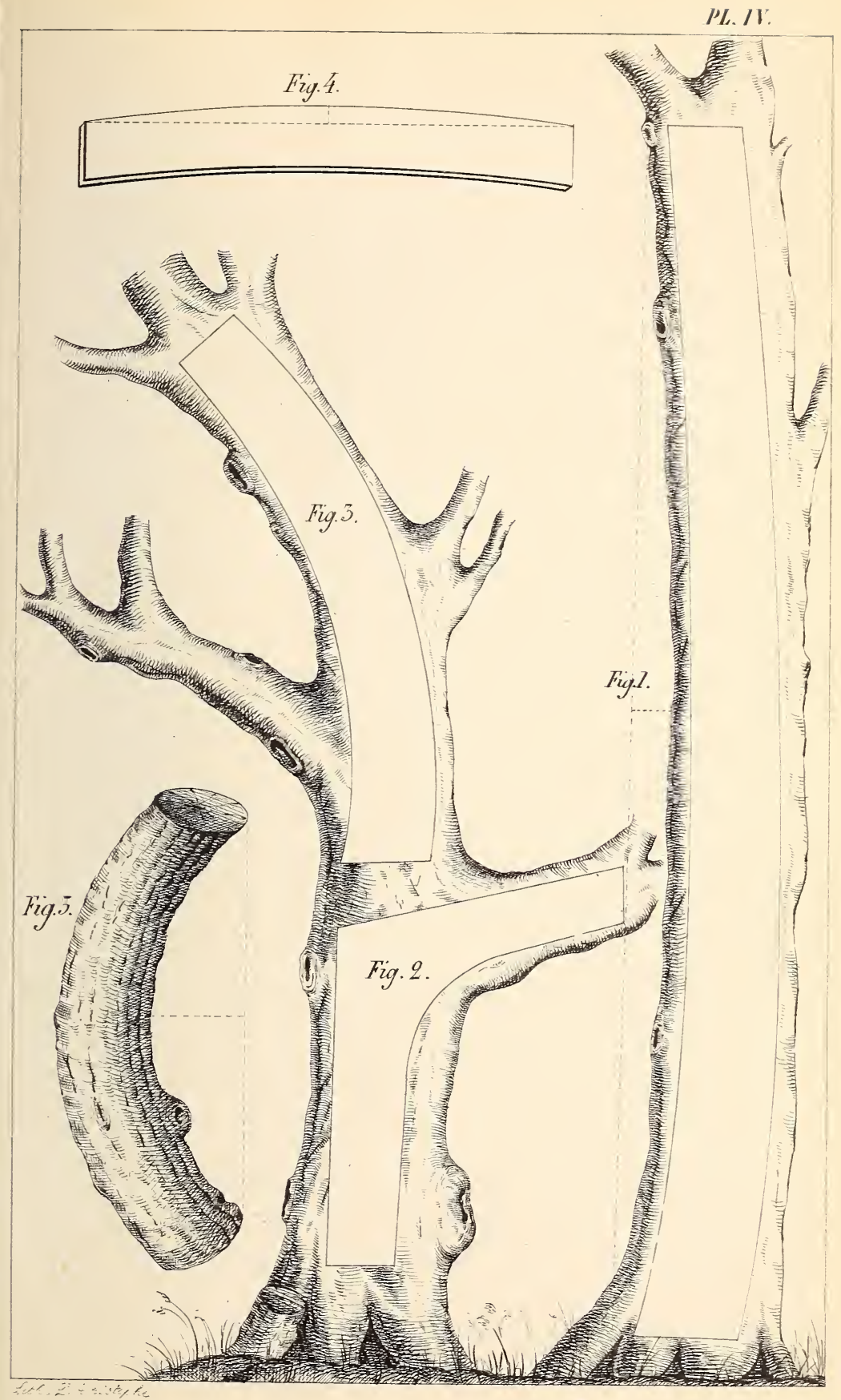



PL. L

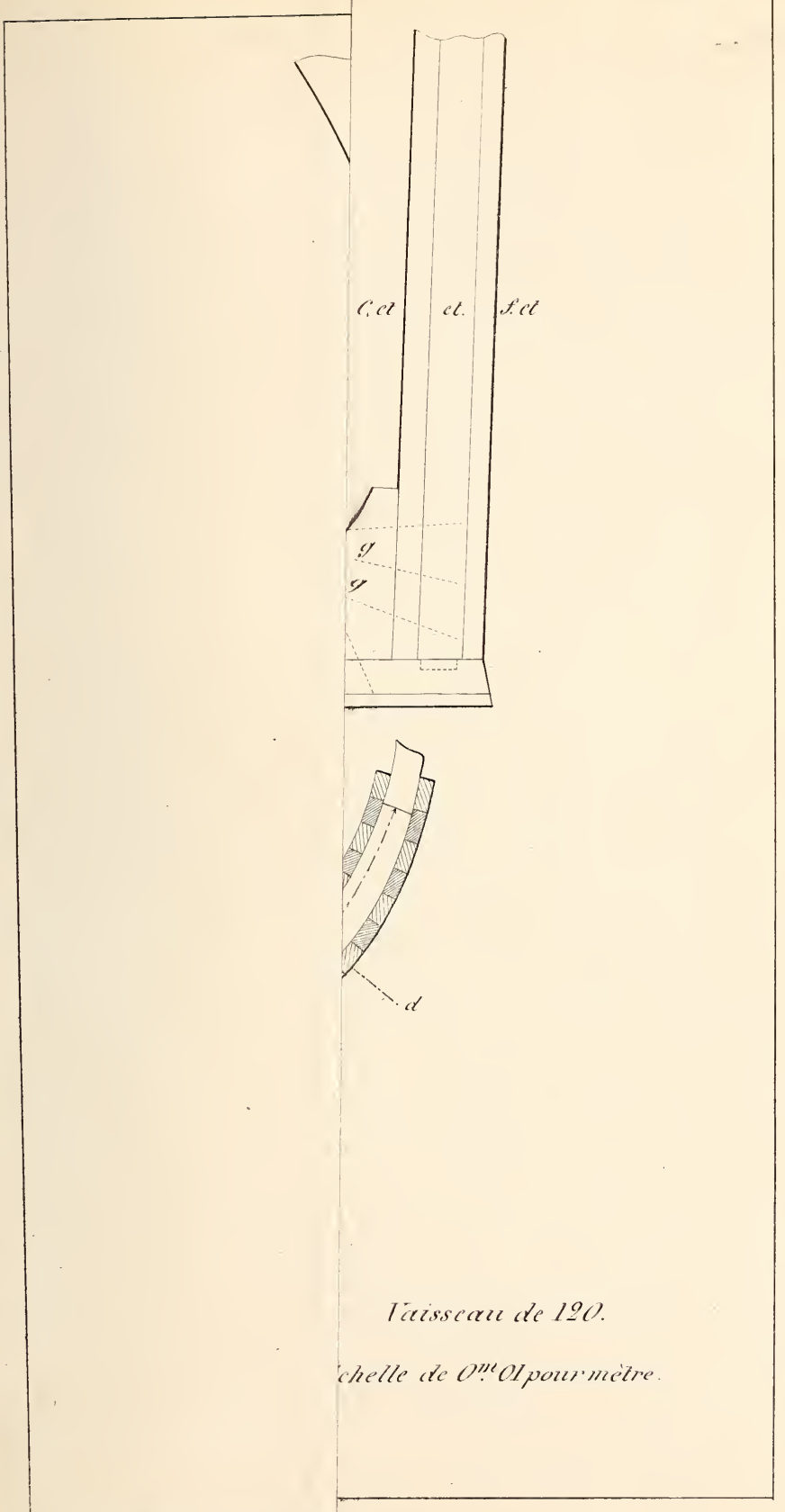




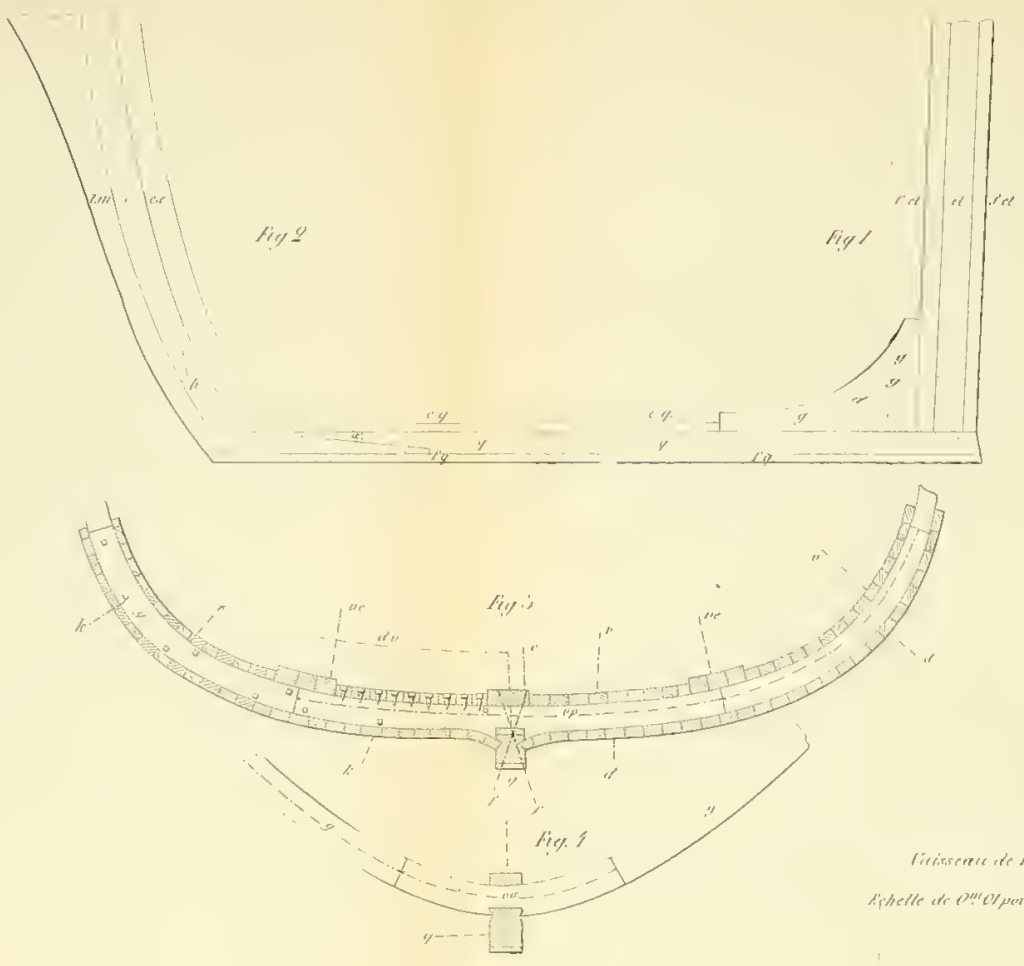



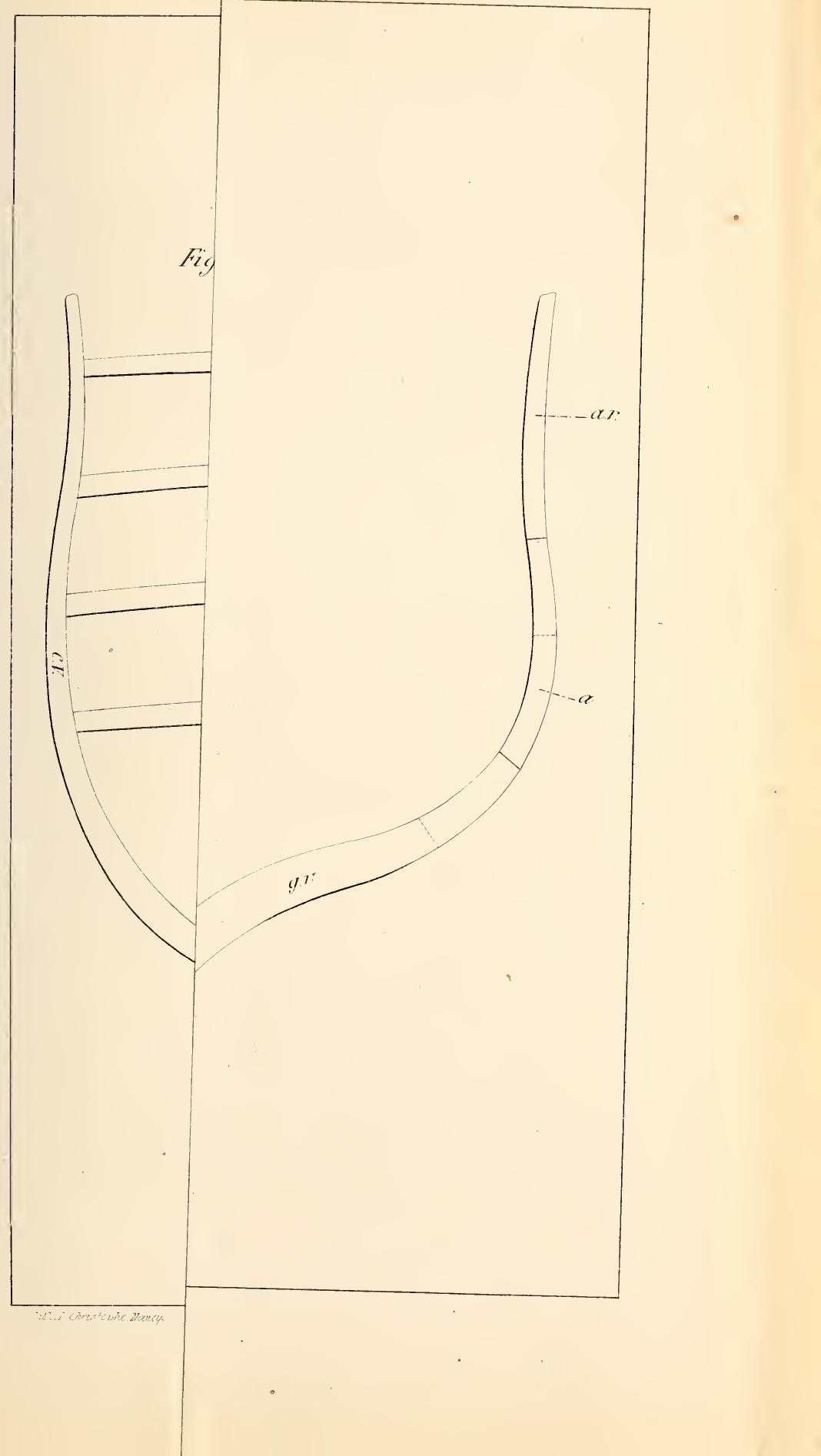





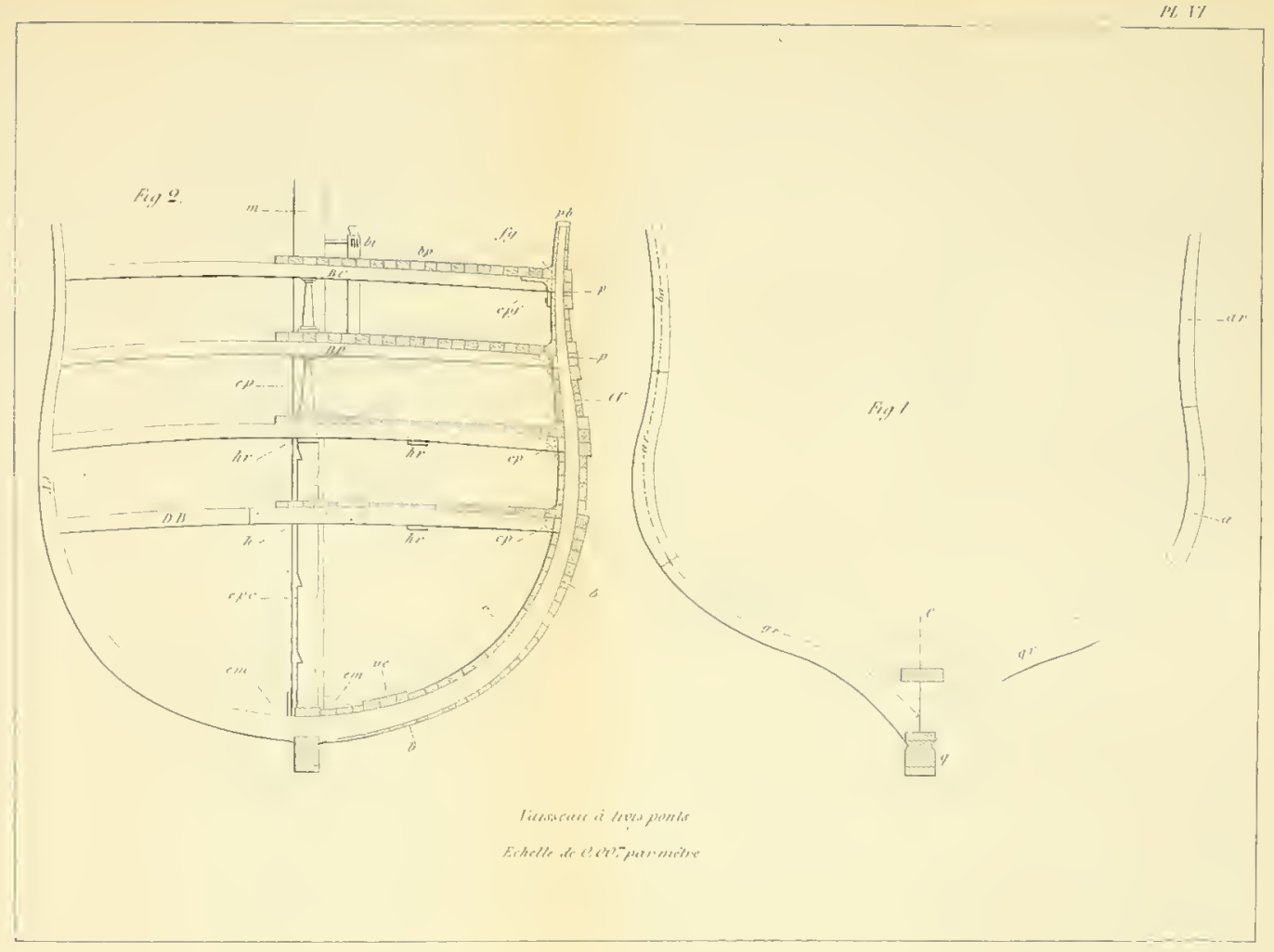





$$
\text { Frig.1. }
$$

$P B$ $P R--\cdots$

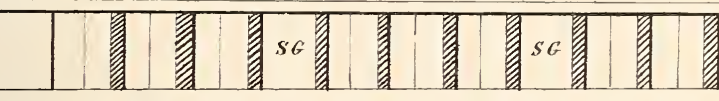
$\prod$ $P R-$ 53

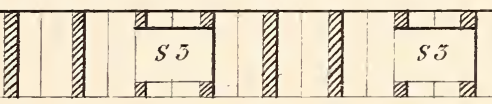
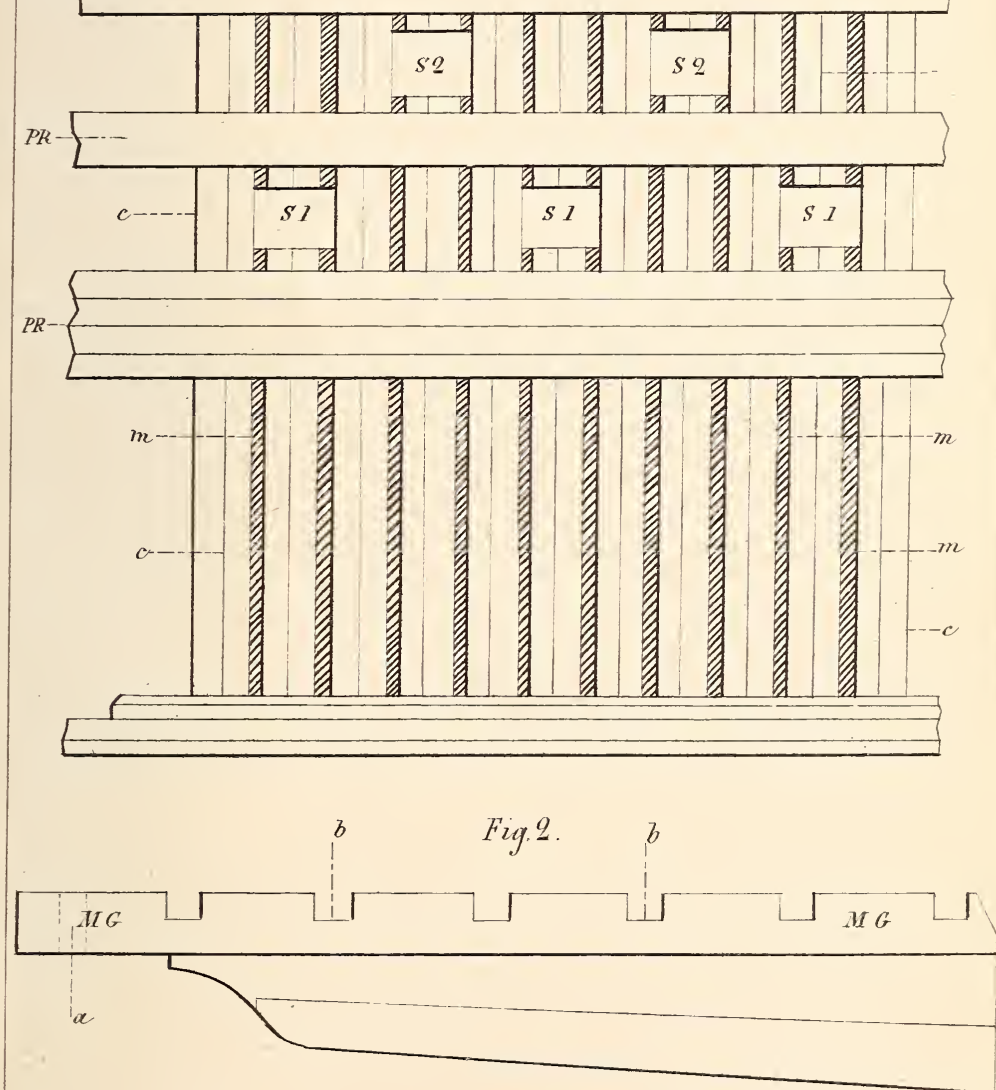




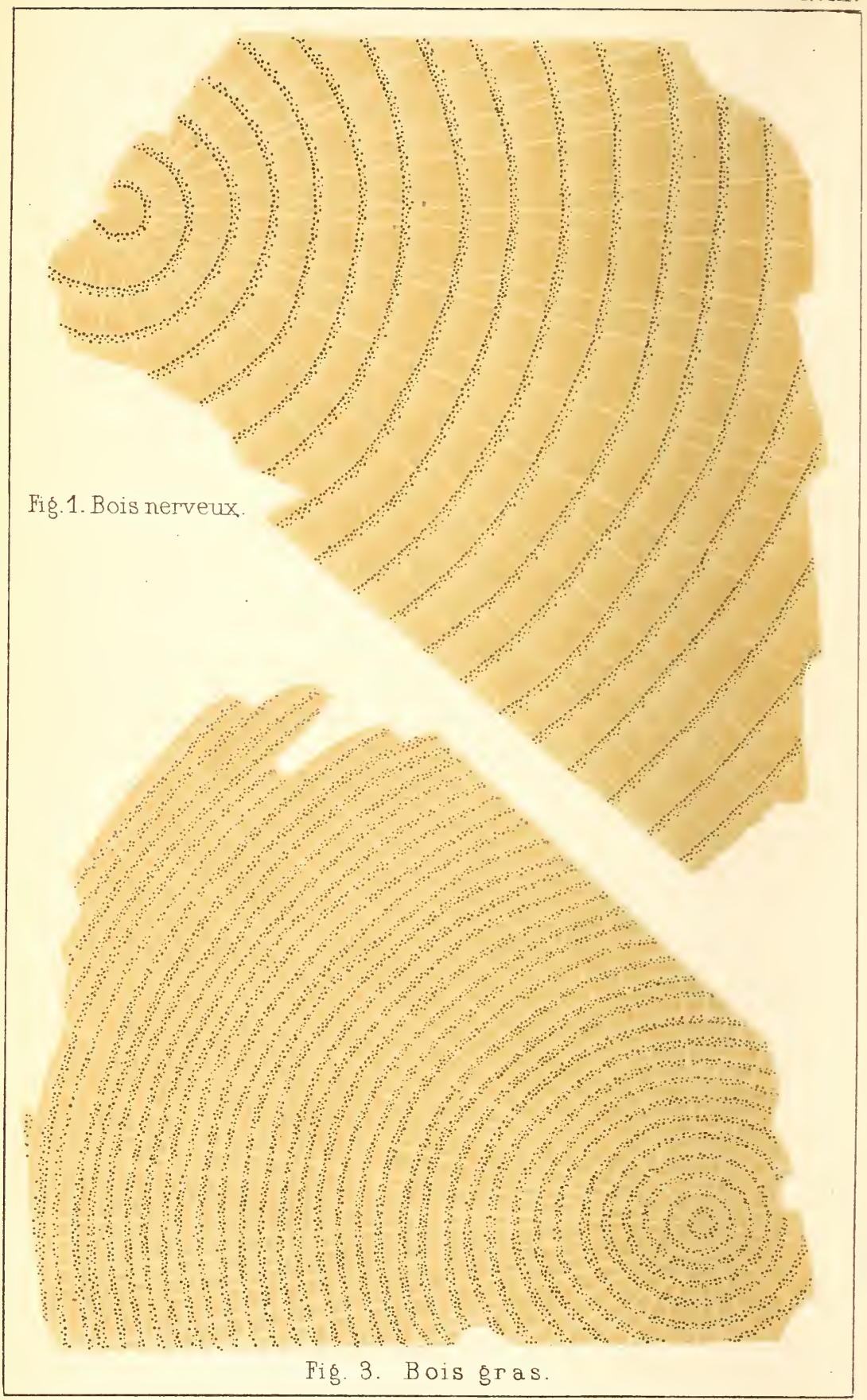

Chromolith. E. Simor à Strasbourǵ 


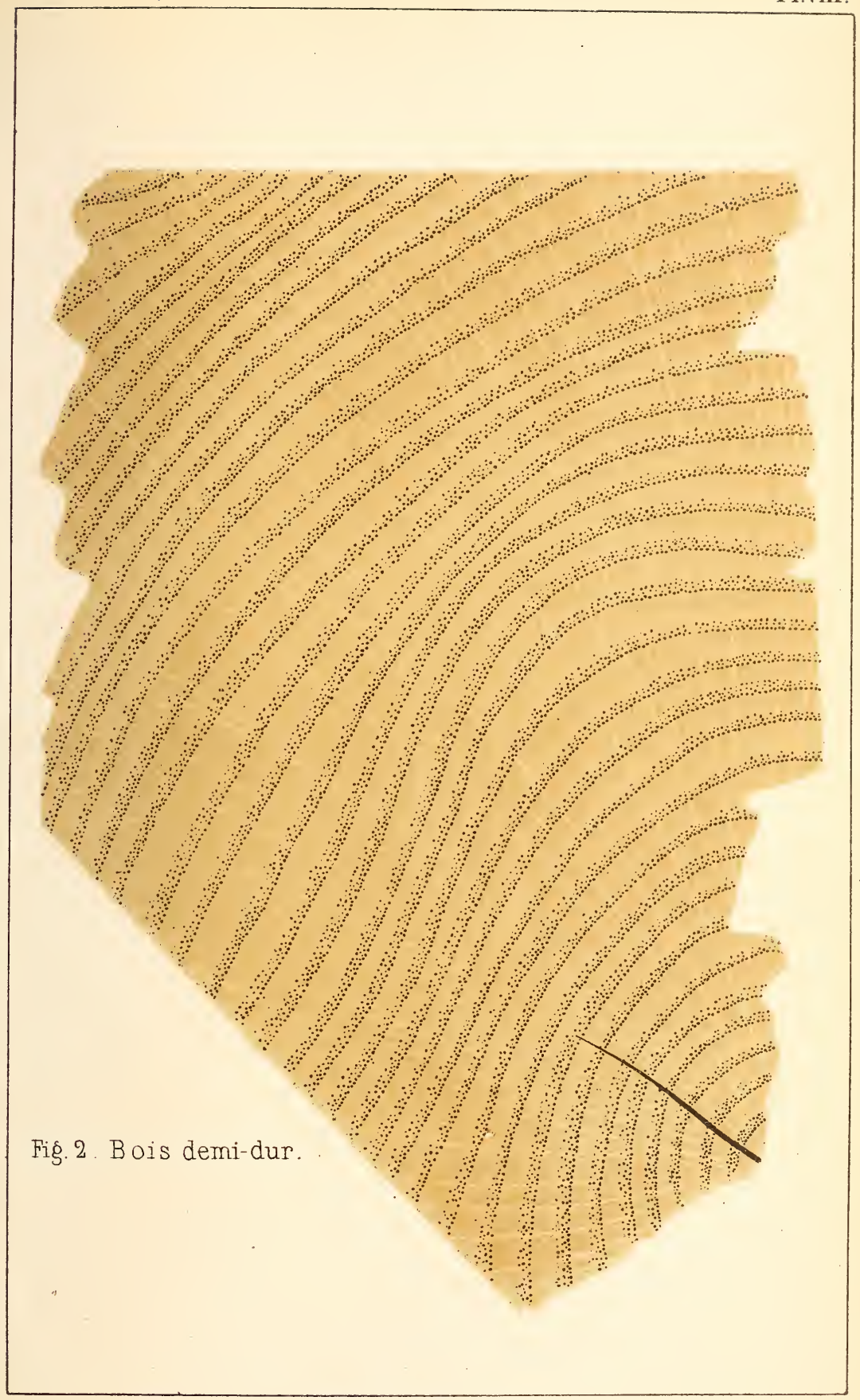

Chromolith. E. Simon à Strasbourś. 


Fig. 1. Gélivures.

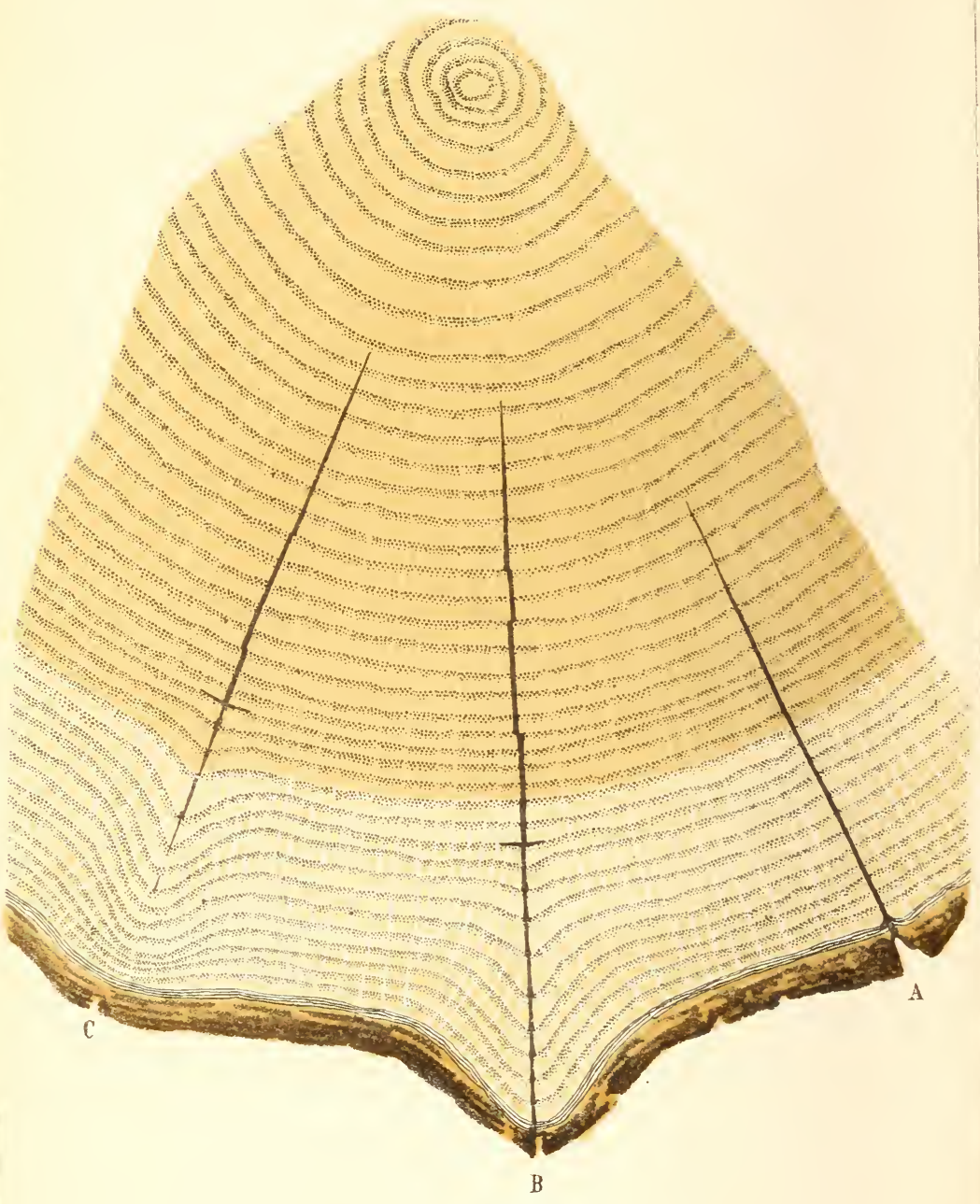

A. Gélivure d'un an.

B. Gélivure de 10 ans, se prolongeant.

r...Gélioure de 15 ans, recouverte. 


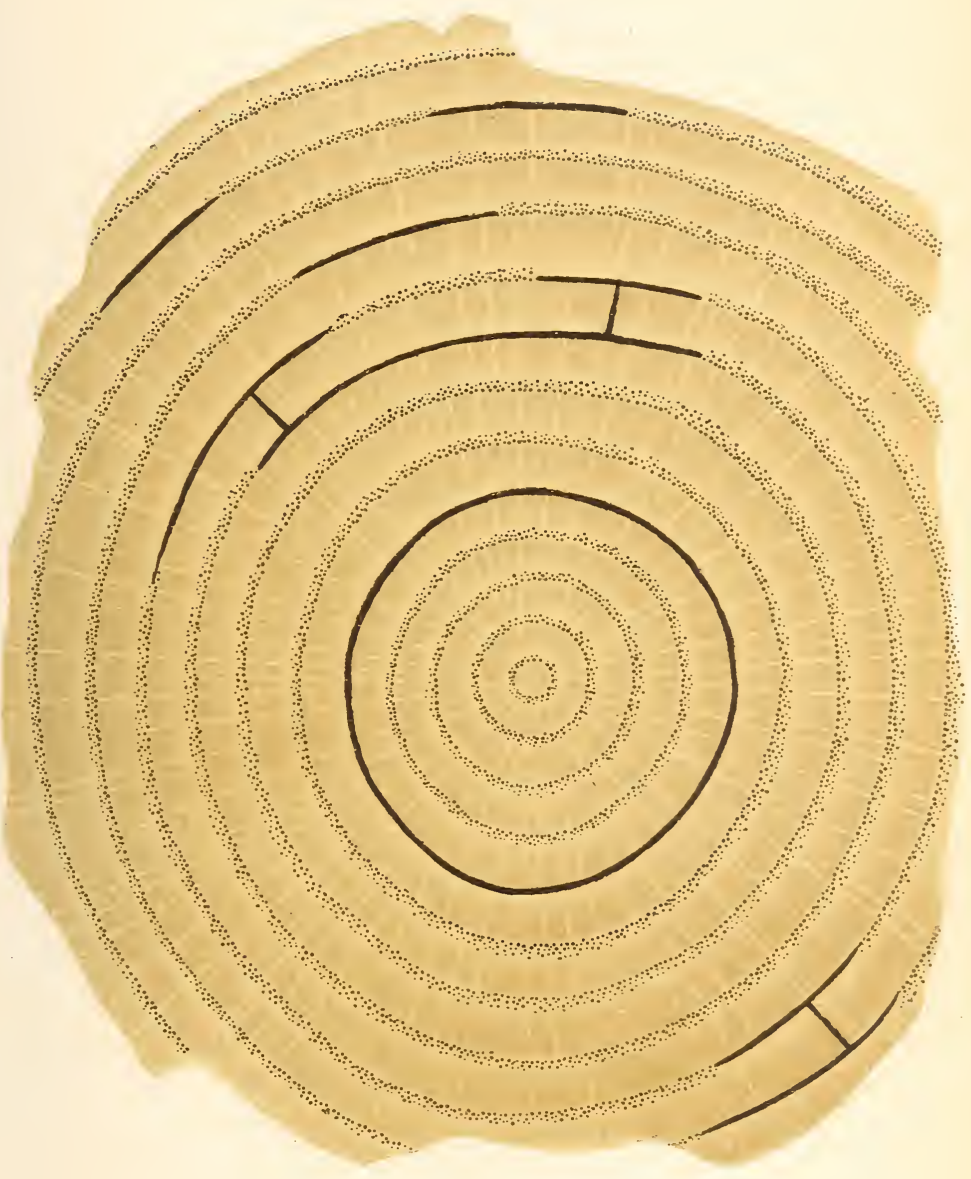

Fig. 2. Roulures 

Fiǵ. 1. Cadranure.

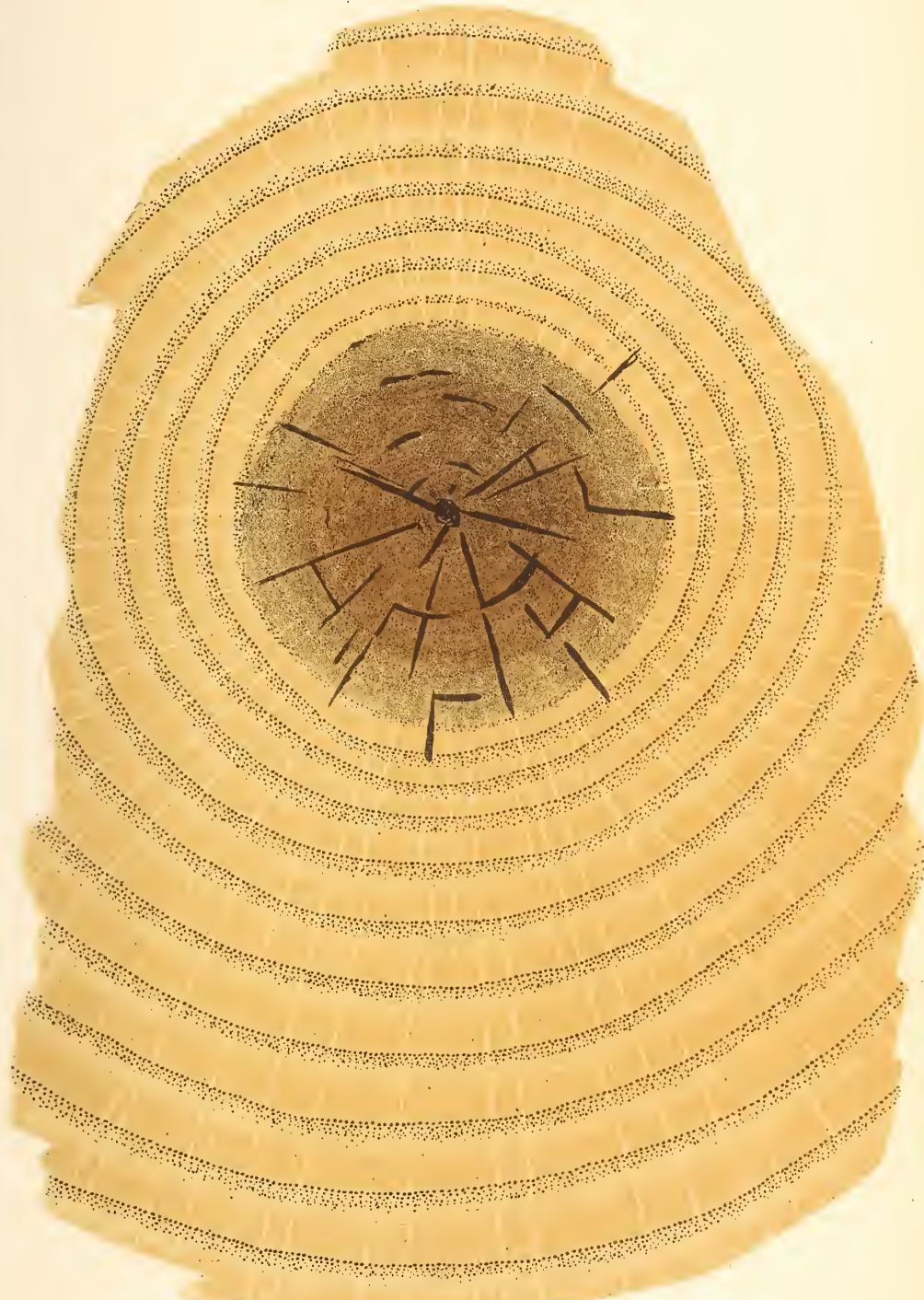





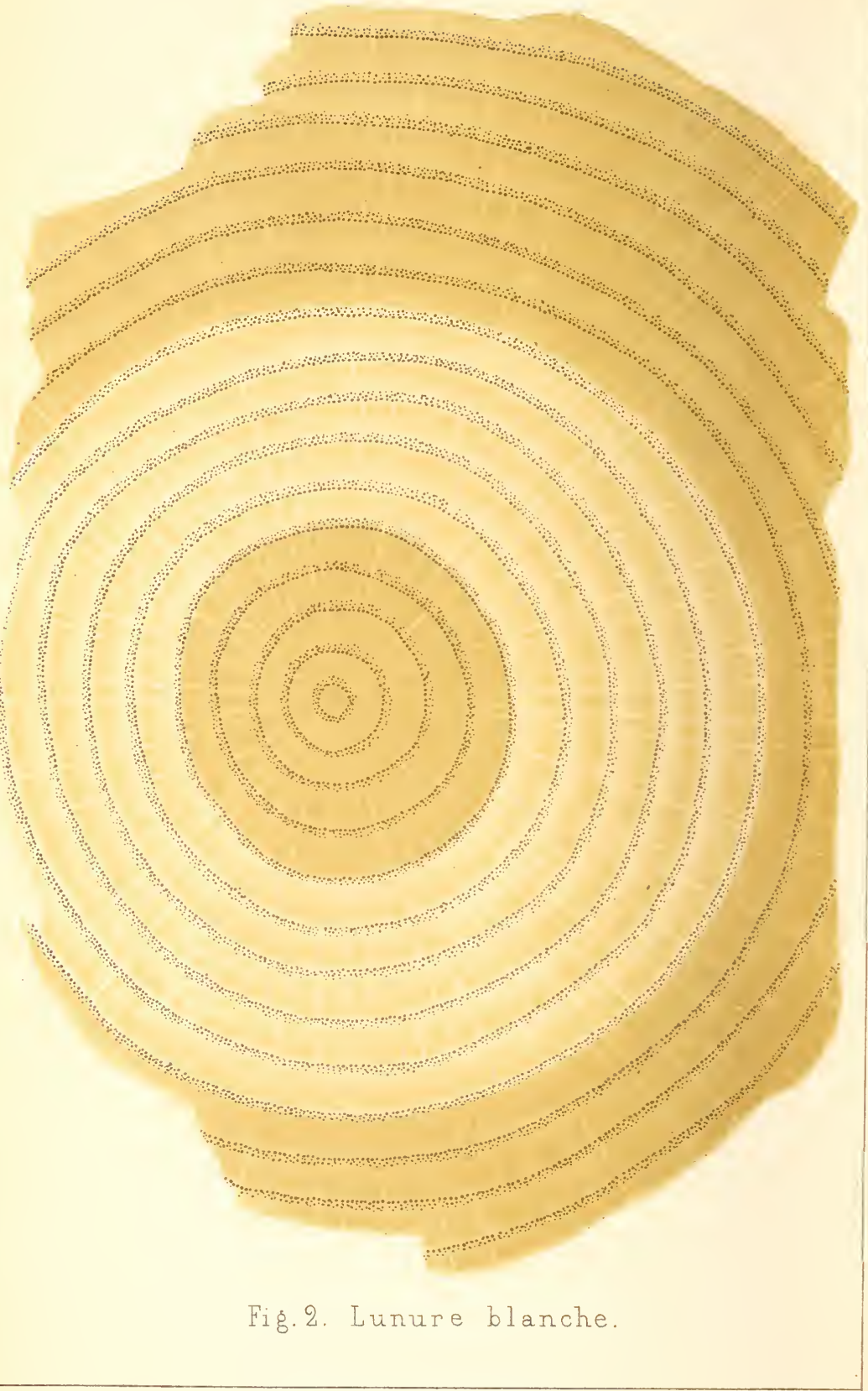


Fig. 2 $2^{\text {bis. }}$ Lunure rousse ou brune.

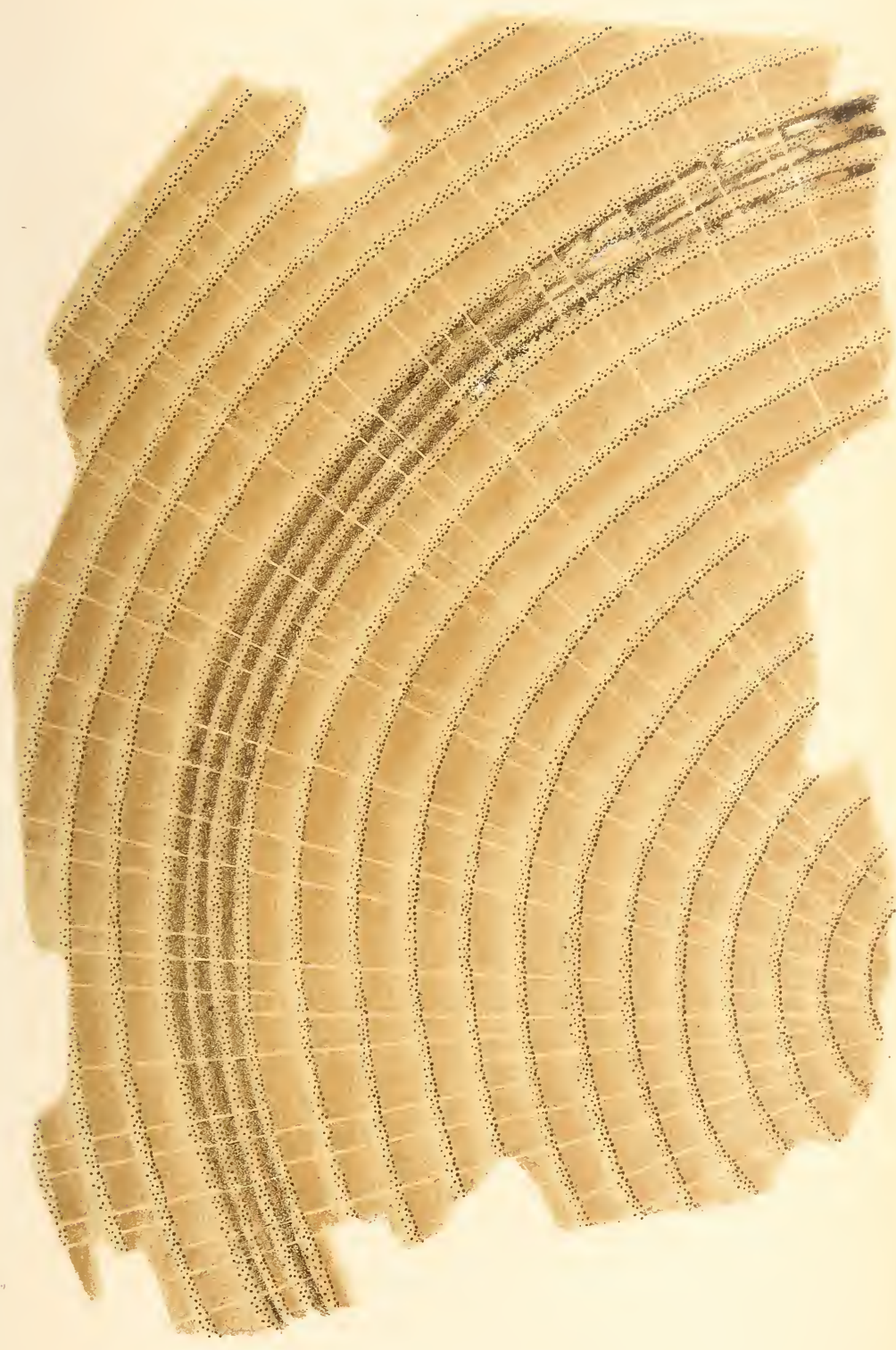






\section{Fig.1. Taches de Gouttières.}

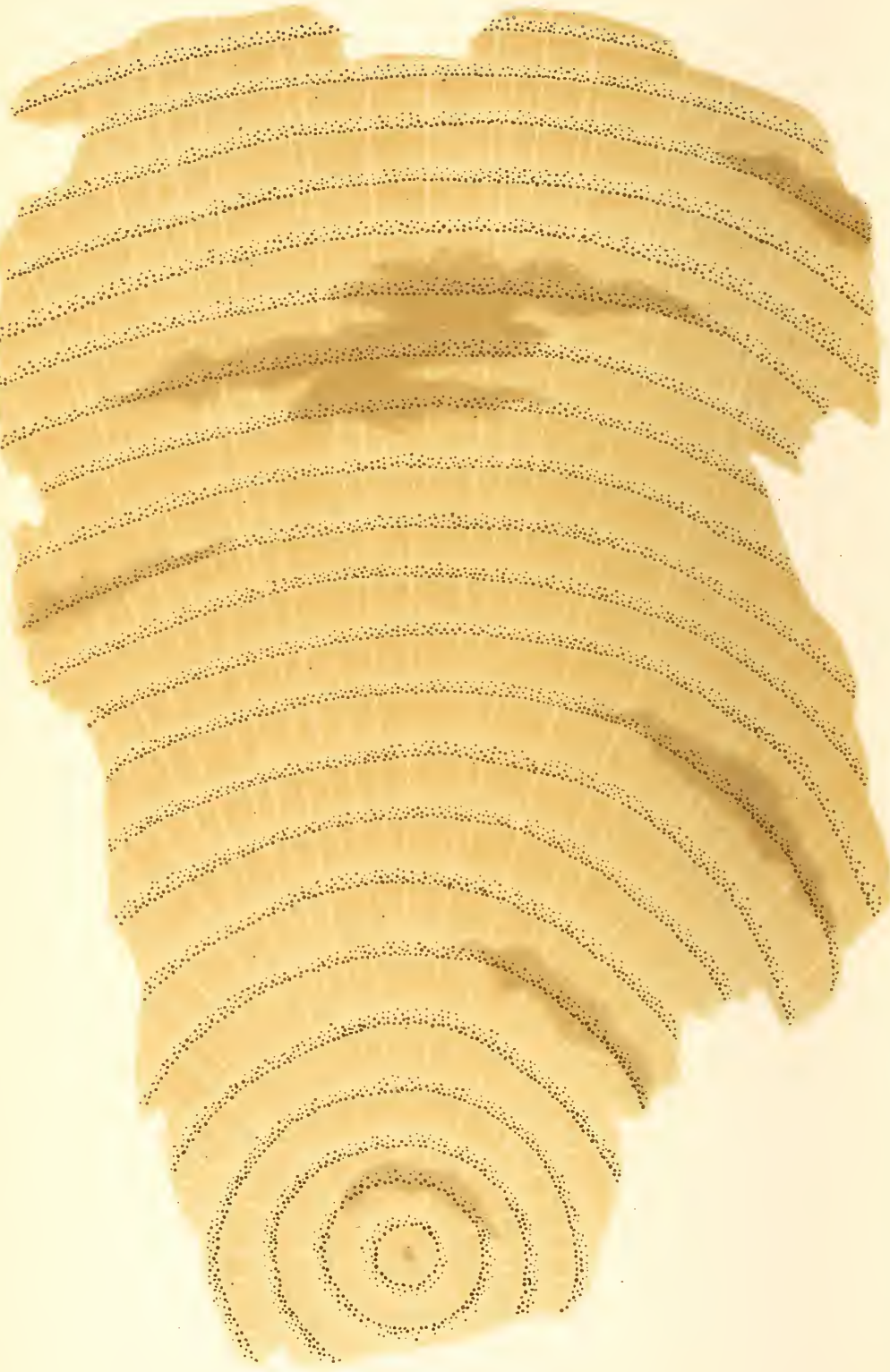


Fig. 1bis. Taches de Gouttière

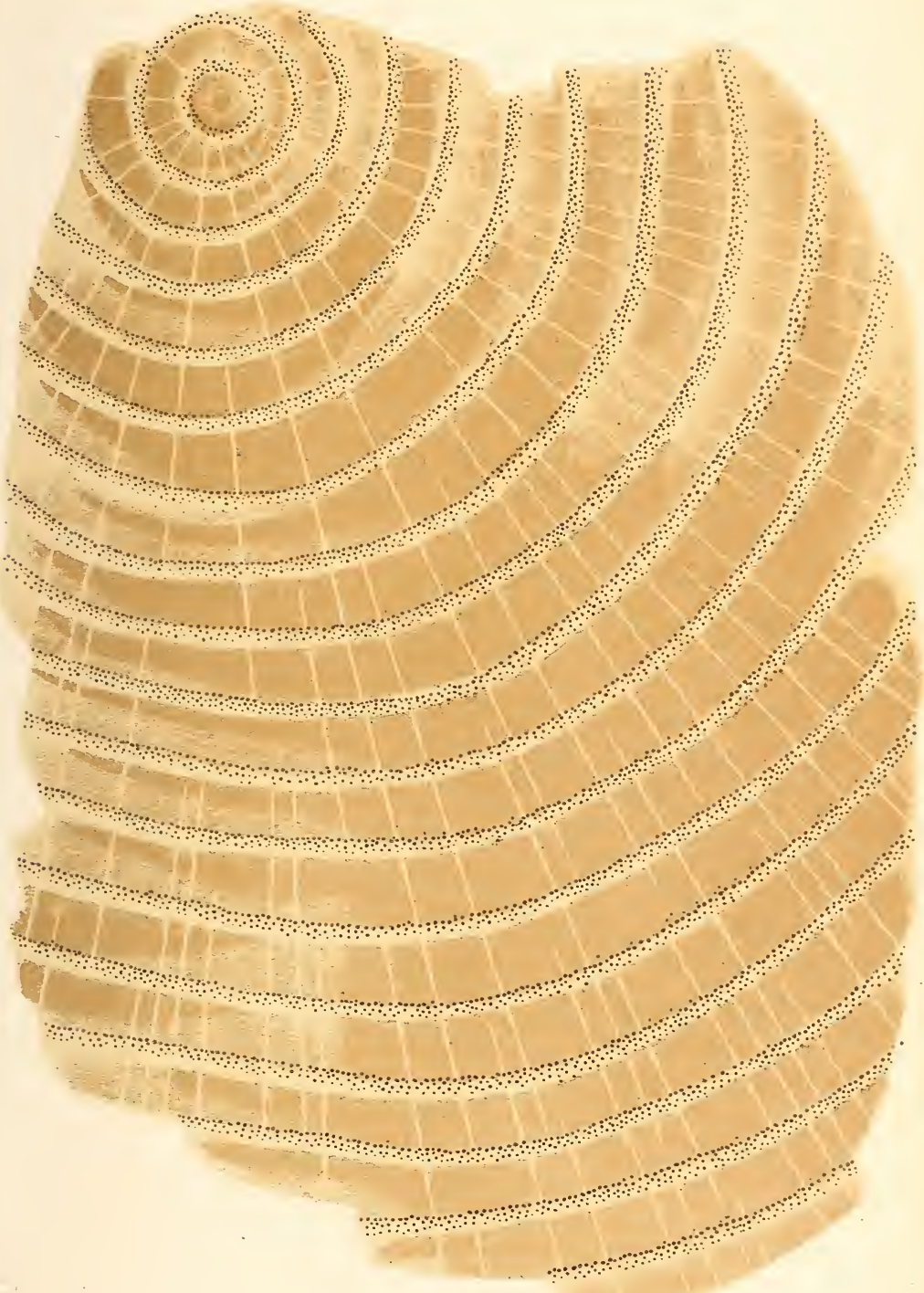





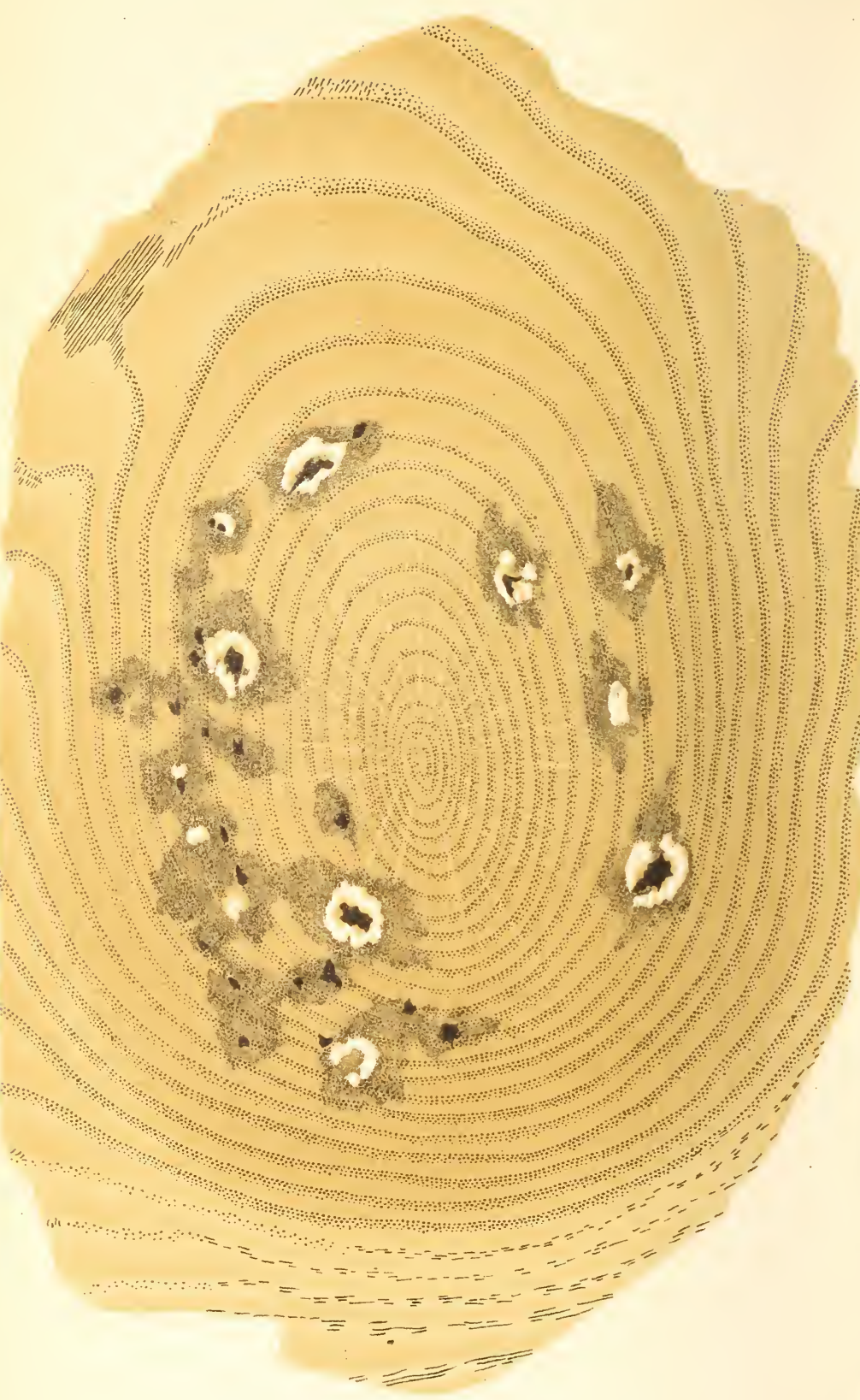

Fig. 2. Nœuds ģâtés (Grisettes) 
Fig. 2. Nœuds gâtés (Grisettes)

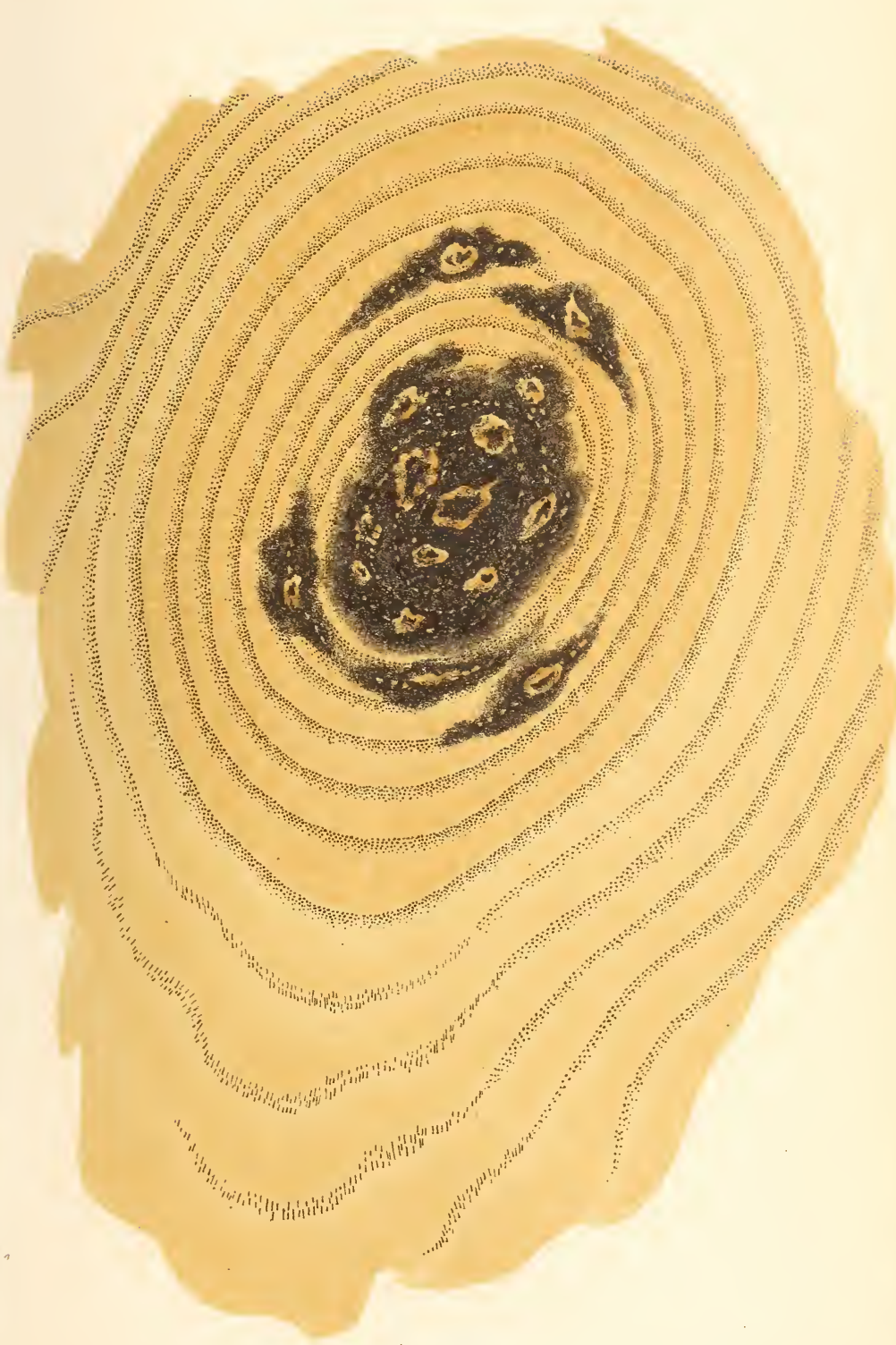



Fig. 1. Frotture.

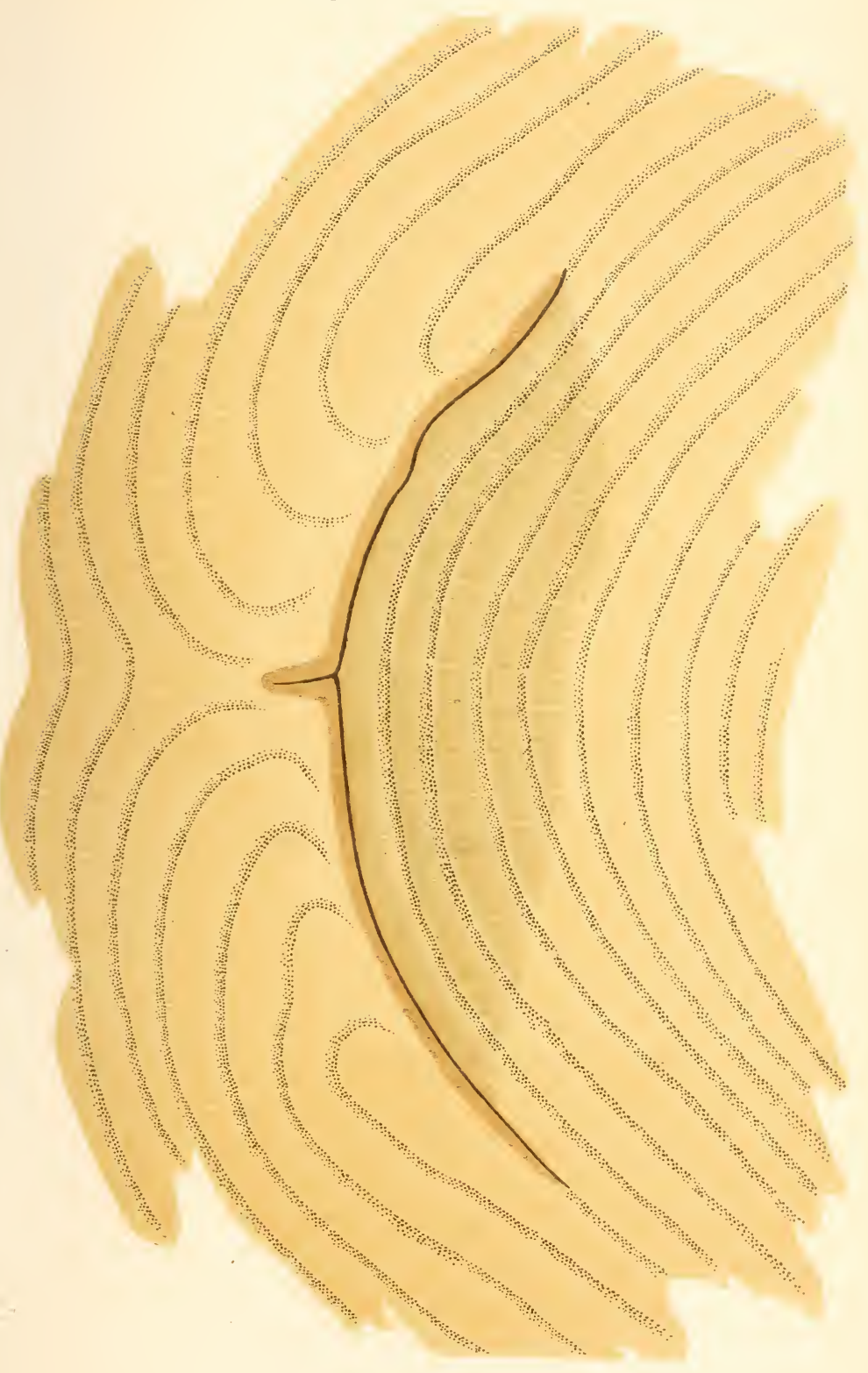





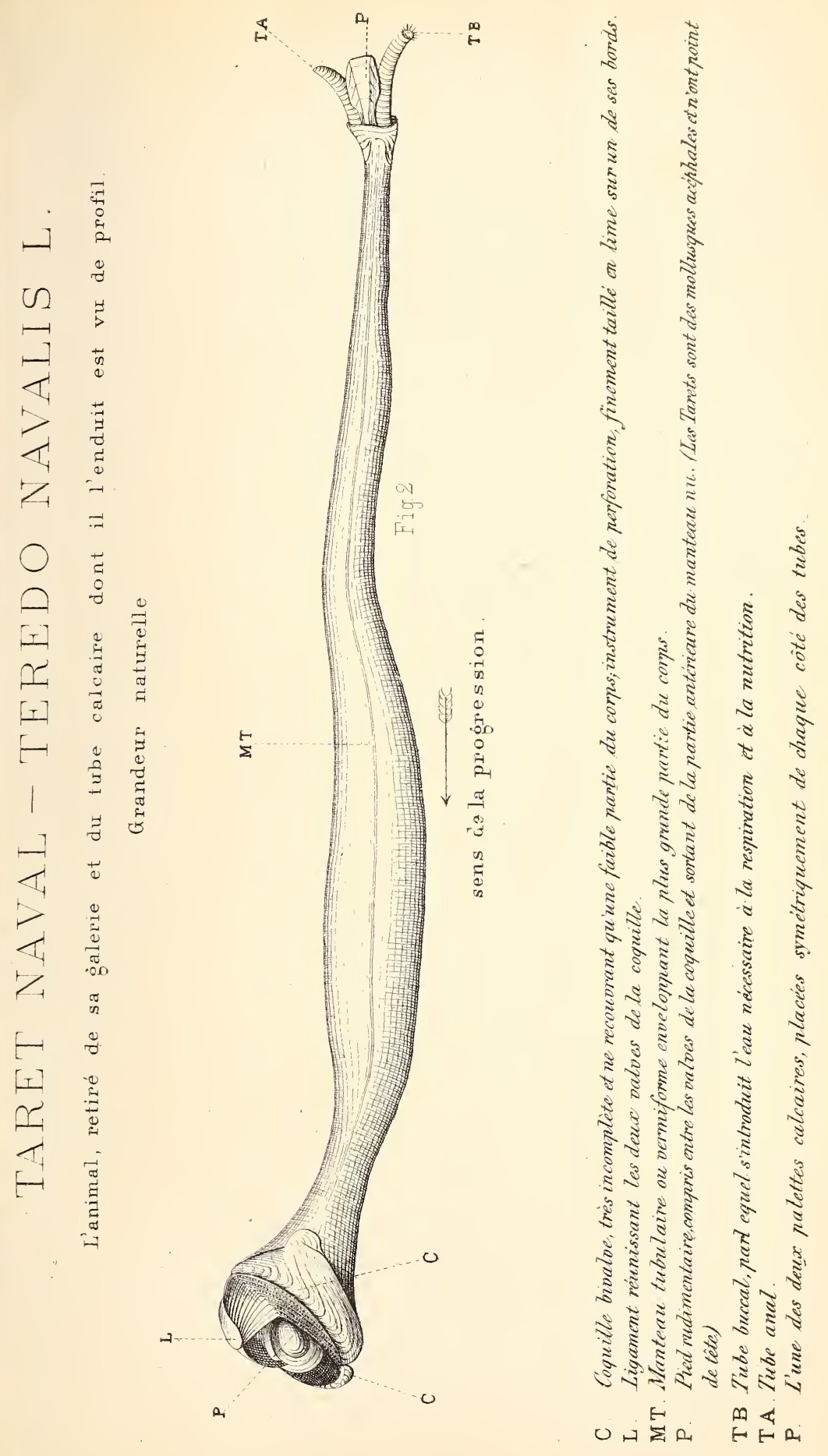






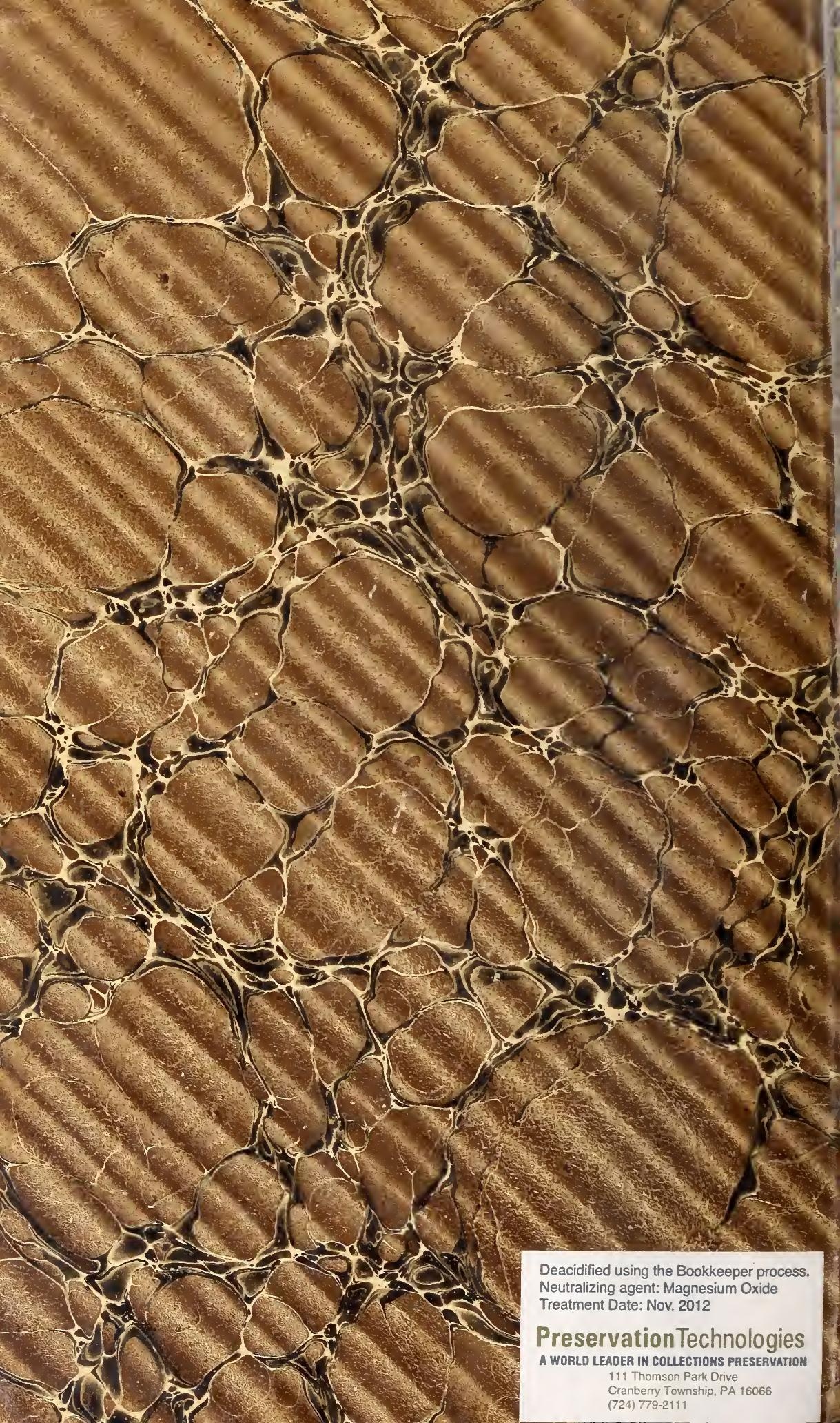


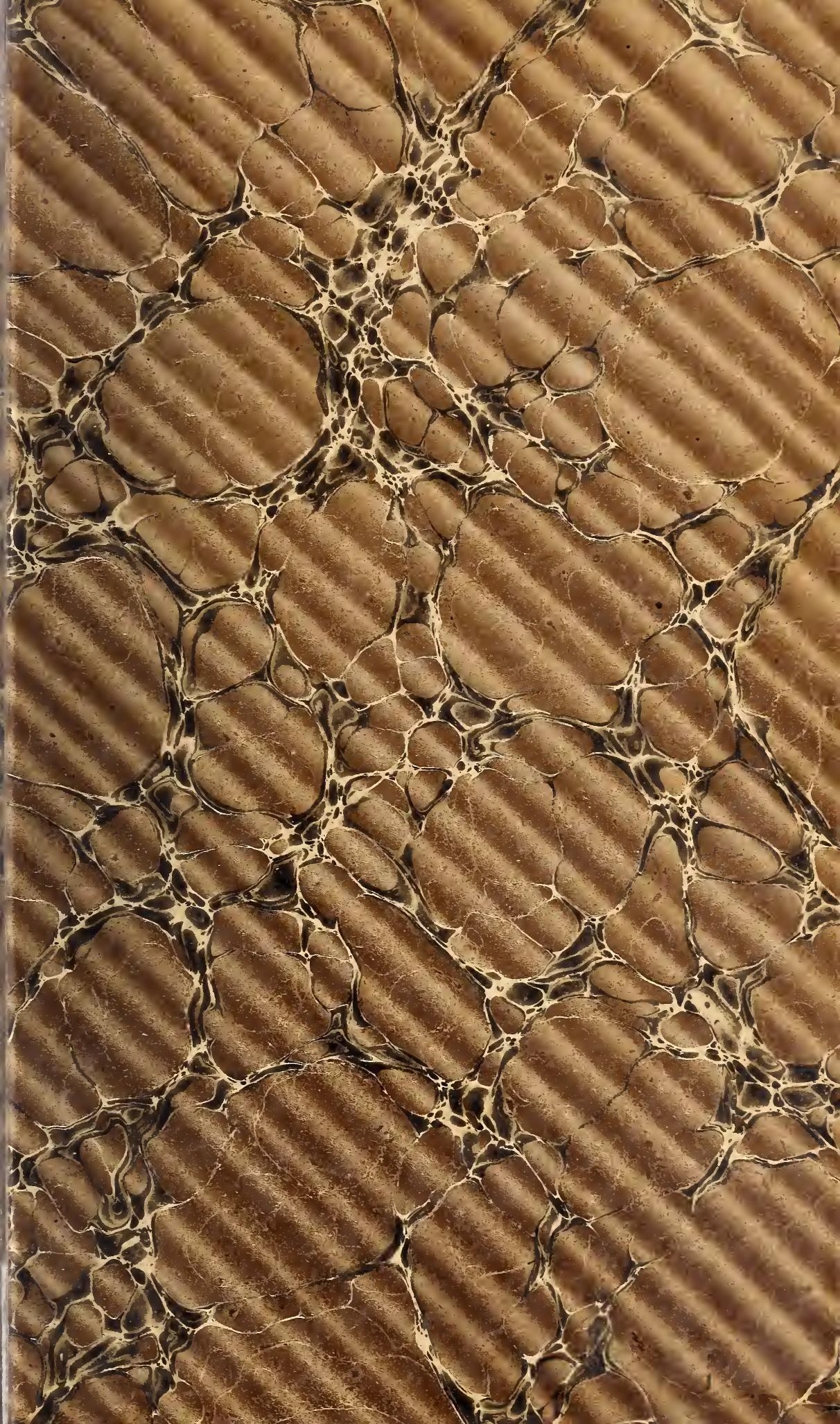


